\title{
Modelos Birnbaum-Saunders usando Equações de Estimação
}

\author{
Aline Barbosa Tsuyuguchi \\ TESE APRESENTADA \\ $\mathrm{AO}$ \\ Instituto de Matemática e Estatística \\ DA \\ Universidade DE SÃo PAUlo \\ PARA \\ OBTENÇÃO DO TÍTULO \\ $\mathrm{DE}$ \\ DOUTOR EM CIÊNCIAS \\ Programa: Estatística \\ Orientador: Prof. Dr. Gilberto Alvarenga Paula \\ Coorientadora: Profa. Dra. Michelli Karinne Barros da Silva
}

Durante o desenvolvimento deste trabalho o autor recebeu auxílio financeiro da CAPES e do $\mathrm{CNPq}$

São Paulo, maio - de 2017 


\section{Modelos Birnbaum-Saunders usando Equações de Estimação}

\footnotetext{
Esta versão da dissertação/tese contém as correções e alterações sugeridas pela Comissão Julgadora durante a defesa da versão original do trabalho, realizada em 12/05/2017. Uma cópia da versão original está disponível no Instituto de Matemática e Estatística da Universidade de São Paulo.
}

Comissão Julgadora:

- Prof. Dr. Gilberto Alvarenga Paula (orientador) - IME-USP

- Prof $^{a}$. Dr ${ }^{a}$. Denise Aparecida Botter - IME-USP

- Prof. Dr. Francisco José de Azevêdo Cysneiros - UFPE

- Prof. Dr. Filidor Edilfonso Vilca Labra - UNICAMP

- Prof ${ }^{\mathrm{a}}$. Dra ${ }^{\mathrm{a}}$. Maria Kelly Venezuela - INSPER 


\section{Agradecimentos}

Primeiramente, agradeço a Deus por nunca me desamparar.

Aos meus pais, Yasutoshi e Marlinda, por todas as oportunidades, pelo apoio incondicional e incentivo, vocês são essenciais. Às minhas irmãs, Ana, Bárbara e Carol por compartilharem comigo as expectativas e ansiedades. À minha sobrinha Aiko, sempre fonte de alegria. A toda minha família, pela torcida entusiasmada e presença constante mesmo quando distante.

Ao professor Gilberto, pela oportunidade de ser sua orientanda, confiança, o inestimável apoio e pelos valiosos conhecimentos transmitidos, muito obrigada.

À professora Michelli, presente na minha trajetória acadêmica desde a graduação, sou muito grata pela dedicação, paciência, amizade e todos os ensinamentos que ultrapassaram as fronteiras da sala de aula.

Ao meu companheiro Fábio, por acreditar em mim quando eu duvidei, pelo amor, compreensão, pelas alegrias compartilhadas, pela tranquilidade e a paz que você traz pra minha vida. Seu auxílio foi muito importante para a conclusão deste trabalho.

Aos amigos que sempre me incentivaram, em especial, Fabiana, Eliza, Roger, Estefa e Cristian, pelo apoio e preciosa amizade que tornaram minha estadia em São Paulo muito mais agradável.

A Terezinha e a professora Kelly Venezuela por auxílios computacionais muito importantes.

A todos os professores do IME/USP que contribuíram para minha formação durante o doutorado. Aos funcionários que fazem parte do IME.

Aos professores que aceitaram participar da banca examinadora pelas valiosas sugestões que contribuíram para a melhoria deste trabalho.

A todos que me ajudaram, de forma direta ou indireta, a passar pelas diversas dificuldades durante esta trajetória, meu mais sincero obrigada. 


\section{Resumo}

Este trabalho de tese tem como objetivo principal propor uma abordagem alternativa para analisar dados Birnbaum-Saunders (BS) correlacionados com base em equações de estimação. Da classe ótima de funções de estimação proposta por Crowder (1987), derivamos uma classe ótima para a análise de dados correlacionados em que as distribuições marginais são assumidas log-BS e log-BSt, respectivamente. Derivamos um processo iterativo para estimação dos parâmetros, métodos de diagnóstico, tais como análise de resíduos, distância de Cook e influência local sob três diferentes esquemas de perturbação: ponderação de casos, perturbação da variável resposta e resposta e perturbação individual de covariáveis. Estudos de simulação são desenvolvidos para cada modelo para avaliar as propriedades empíricas dos estimadores dos parâmetros de localização, forma e correlação. A abordagem apresentada é discutida em duas aplicações: o primeiro exemplo referente a um banco de dados sobre a produtividade de capital público nos 48 estados norte-americanos contíguos de 1970 a 1986 e o segundo exemplo referente a um estudo realizado na Escola de Educação Física e Esporte da Universidade de São Paulo (USP) durante 2016 em que 70 corredores foram avaliados em corridas em esteiras em três períodos distintos.

Palavras-chave: distribuição Birnbaum-Saunders, dados correlacionados, equações de estimação. 


\section{Abstract}

The aim of this thesis is to propose an alternative approach to analyze correlated BirnbaumSaunders (BS) data based on estimating equations. From the optimal estimating functions class proposed by Crowder (1987), we derive an optimal class for the analysis of correlated data in which the marginal distributions are assumed either log-BS or log-BS-t. It is derived an iterative process, diagnostic procedures such as residual analysis, Cook's distance and local influence under three different perturbation schemes: case-weights, response variable perturbation and single-covariate perturbation. Simulation studies to assess the empirical properties of the parameters estimates are performed for each proposed model. The proposed methodology is discussed in two applications: the first one on a data set of public capital productivity of the contiguous 48 USA states, from 1970 to 1986, and the second data set refers to a study conducted in the School of Physical Education and Sport of the University of São Paulo (USP), during 2016, in which 70 runners were evaluated in running machines races in three periods.

Keywords: Birnbaum-Saunders distribution, correlated data, estimating equations. 


\section{Sumário}

1 Introdução $\quad \mathbf{1}$

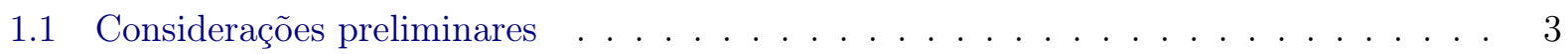

1.1.1 Distribuição Birnbaum-Saunders . . . . . . . . . . . . . . . . . 3

1.1 .2 Distribuição $\operatorname{log-Birnbaum-Saunders~\ldots .~.~.~.~.~.~.~.~.~.~.~.~.~} 5$

1.1.3 Distribuição Birnbaum-Saunders generalizada . . . . . . . . . . . . . . 6

1.1.4 Distribuição $\log -\mathrm{BS}-t \ldots \ldots \ldots \ldots \ldots$. . . . . . . . . . . 7

1.1.5 Modelo de regressão $\operatorname{log-Birnbaum-Saunders~\ldots ~.~.~.~.~.~.~.~.~.~.~.~.~} 8$

1.1.6 Modelos mistos . . . . . . . . . . . . . . . . . . . . . . . 9

1.1.7 Distribuição Birnbaum-Saunders multivariada generalizada . . . . . . . . . 10

1.1 .8 Funções de estimação . . . . . . . . . . . . . . . . . . . . . . . . 11

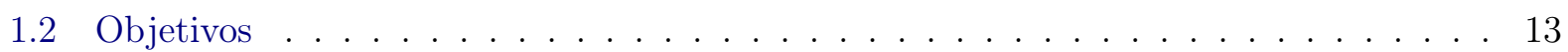

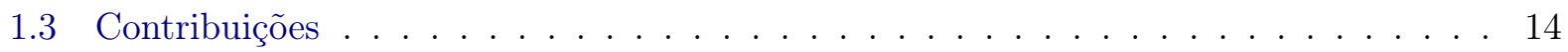

1.4 Organização do trabalho . . . . . . . . . . . . . . . . . . . . 14

2 Equações de Estimação para modelos de regressão log-BS $\quad 17$

2.1 Notação . . . . . . . . . . . . . . . . . . . . . . . . . . . . . . 17

2.2 Equações de estimação para a média . . . . . . . . . . . . . . . . 18

2.2.1 Derivação da função de estimação . . . . . . . . . . . . . . . . . . . . 18

2.2 .2 Algoritmo de estimação . . . . . . . . . . . . . . . . . . . . . . 19

2.2 .3 Inferência . . . . . . . . . . . . . . . . . . . . 22

2.2 .4 Estudo de simulação . . . . . . . . . . . . . . . . . . . . . 23

2.2 .5 Métodos de diagnóstico . . . . . . . . . . . . . . . . . . . 24

2.2 .6 Aplicação . . . . . . . . . . . . . . . . . . . . . . . . . . . . . . . . . . . . 32

3 Equações de Estimação para modelos de regressão log-BS- $t \quad 39$

3.1 Notação . . . . . . . . . . . . . . . . . . . . . . . . . . . . . . . . . . . . 39

3.2 Equações de estimação para a média . . . . . . . . . . . . . . . . . . . . . . . . . . . . . . . . .

3.2 .1 Derivação da função de estimação . . . . . . . . . . . . . . . . . . . . . . 40

3.2 .2 Estimação . . . . . . . . . . . . . . . . . . . . . . . . . . 42

3.2 .3 Processo iterativo . . . . . . . . . . . . . . . . . . . . 43

3.2 .4 Inferência . . . . . . . . . . . . . . . . . . . 43

3.2 .5 Estudo de simulação . . . . . . . . . . . . . . . . . . . . . 43

3.2 .6 Métodos de diagnóstico . . . . . . . . . . . . . . . . . . . . 44

3.2 .7 Aplicação . . . . . . . . . . . . . . . . . . . . 45 
4 Equações de Estimação Conjuntas para a Média e Forma

4.1 Equação de estimação conjunta para o modelo log-BS. . . . . . . . . . . . . . . . 53

4.1 .1 Derivação de função de estimação conjunta . . . . . . . . . . . . . . . . . . 53

4.1 .2 Algoritmo de estimação . . . . . . . . . . . . . . . . . . 56

4.1 .3 Inferência . . . . . . . . . . . . . . . . . . . . 57

4.1 .4 Estudo de simulação . . . . . . . . . . . . . . . . . . . . 58

4.1 .5 Métodos de diagnóstico f . . . . . . . . . . . . . . . . . . . . . . . . 59

4.2 Equação de estimação conjunta para o modelo log-BS-t . . . . . . . . . . . . . . . 63

4.2.1 Derivação de função de estimação conjunta . . . . . . . . . . . . . . . . . . . 63

4.2 .2 Algoritmo de estimação . . . . . . . . . . . . . . . . . . . 67

4.2 .3 Estudo de Simulação . . . . . . . . . . . . . . . . . . . . . . . . . . 68

4.2 .4 Métodos de diagnóstico f . . . . . . . . . . . . . . . . 68

5 Considerações Finais $\quad 71$

5.1 Trabalhos futuros . . . . . . . . . . . . . . . . . . . . . 71

$\begin{array}{ll}\text { A } & 73\end{array}$

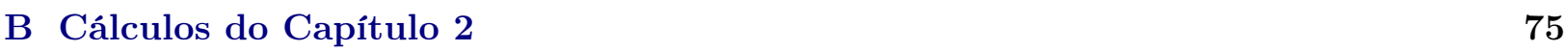

B.1 Função de estimação para o parâmetro de localização . . . . . . . . . . . . . . . . . . 75

B.2 Matrizes de sensibilidade e variabilidade . . . . . . . . . . . . . . . 77

B.3 Influência conformal sob homogeneidade do parâmetro de forma . . . . . . . . . . . . 81

B.3.1 Perturbação da variável resposta . . . . . . . . . . . . . . . . . . 81

B.3.2 Perturbação individual das covariáveis . . . . . . . . . . . . . . . . . . . 82

$\begin{array}{lll}\text { C } & \text { Tabelas referentes às simulações do Capítulo } 2 & 85\end{array}$

$\begin{array}{ll}\text { D Cálculos do Capítulo } 3 & 89\end{array}$

D.1 Função de estimação para o parâmetro de localização . . . . . . . . . . . . . . . . . 89

D.2 Matrizes de sensibilidade e variabilidade . . . . . . . . . . . . . . . . . . . 91

D.3 Influência conformal sob homogeneidade do parâmetro de forma . . . . . . . . . . . . 94

D.3.1 Perturbação individual das covariáveis . . . . . . . . . . . . . . . . . . . 94

$\begin{array}{lll}\text { E } & \text { Tabelas referentes às simulações do Capítulo } 3 & 97\end{array}$

$\begin{array}{llr}\text { F Cálculos do Capítulo } 4 & 107\end{array}$

F.1 Função de estimação conjunta para o modelo heterogêneo log-BS . . . . . . . . . . 107

F.1.1 Derivação da função de estimação para o modelo do parâmetro de forma . 107

F.1.2 Derivação da Função de Estimação Conjunta . . . . . . . . . . . . . . . . . . 109

F.1.3 Derivação das matrizes de variabilidade e sensibilidade . . . . . . . . . . . . . 110

F.1.4 Influência conformal sob heterogeneidade do parâmetro de forma . . . . . . . 112

F.2 Função de estimação conjunta para o modelo heterogêneo log-BS-t . . . . . . . . . . 115

F.2.1 Derivação das matrizes de variabilidade e sensibilidade . . . . . . . . . . . . 115

F.2.2 Influência conformal sob heterogeneidade do parâmetro de forma . . . . . . . 117 
G Tabelas referentes às simulações do Capítulo 4

Referências Bibliográficas 


\section{Capítulo 1}

\section{Introdução}

A distribuição Birnbaum-Saunders (BS) é uma distribuição de tempo de vida (life distribution) que relaciona o tempo até a ocorrência de falha com algum dano cumulativo que é assumido gaussiano. A principal vantagem das pesquisas relacionadas a essa distribuição é o melhor ajuste nos percentis mais baixos ou mais altos da distribuição (onde há o maior interesse dos analistas) quando comparada às demais distribuições de vida. Para maiores detalhes, veja Birnbaum e Saunders (1969) e Leiva (2016). Nas últimas décadas tem sido crescente o interesse pelo estudo da distribuição BS e muitas pesquisas utilizando essa distribuição surgiram, por exemplo, Desmond (1985) que relaxou algumas suposições feitas por Birnbaum e Saunders (1969) e apresentou uma derivação mais geral baseada em um modelo biológico. Além disso, Desmond (1986) estabeleceu uma conexão bastante útil entre a distribuição gaussiana inversa e a distribuição BS. Uma outra referência importante é o artigo Rieck e Nedelman (1991) no qual é proposto um modelo log-linear para a distribuição Birnbaum-Saunders e desenvolvido métodos de estimação. O modelo log-linear proposto vêm sendo empregado em uma grande quantidade de estudos, como por exemplo Tsionas (2001) que discute a estimação Bayesiana para o modelo, Galea et al. (2004) que apresentaram vários métodos de diagnóstico para modelos log-linear BS, Leiva et al. (2007) que estudaram o modelo de regressão log-BS com observações censuradas. Díaz-García e Leiva (2005) apresentaram uma generalização da distribuição BS, que foi derivada com base em distribuições de contornos elípticos e foi denominada de distribuição Birnbaum-Saunders generalizada (BSG). A principal vantagem da distribuição BSG é ser mais flexível por admitir vários graus de curtose (quando comparada com a distribuição BS), veja Sanhueza et al. (2008). Barros et al. (2008) propuseram então, uma nova classe de modelos de regressão em que os erros seguem uma distribuição log-BSG baseada no modelo t-Student. Santos-Neto et al. (2012) propuseram uma reparametrização da distribuição BS, que denoraminaram Birnbaum-Saunders reparametrizada (BSR), Santos-Neto et al. (2014) forneceram alguns resultados em momentos, estimação e inferência para BSR de Santos-Neto et al. (2012) e Leiva et al. (2014) propuseram um modelo de regressão BSR em que a média é relacionada ao preditor linear através de uma função de ligação.

Embora a distribuição BS tenha sido originada a partir da fadiga de materiais, as distribuições BS e BSG vêm sendo amplamente aplicadas em diversas áreas do conhecimento, tais como: engenharia, medicina, contaminação atmosférica, qualidade da água, catástrofes naturais, negócios, finanças, indústria, ciência de gestão e controle de qualidade. Veja por exemplo: Leiva et al. (2007) 
onde a distribuição BS foi utilizada para modelar o tempo de sobrevivência de pacientes com múltiplos mielomas; Balakrishnan et al. (2007) usaram a distribuição BS para modelar o tempo do início da execução de um software até a a falha do mesmo; Leiva et al. (2008) apresentaram a distribuição BSG como um modelo útil para descrever dados de poluição, Bhatti (2010) e Marchant et al. (2013) aplicaram a distribuição BS em dados financeiros de alta frequência e Paula et al. (2012) modelaram dados oriundos de reclamações de uma seguradora, usando a BSG.

Mesmo que muitas vezes a suposição de independência não seja razoável, a maioria dos trabalhos desenvolvidos no âmbito de modelos BS e BSG assume independência entre as unidades experimentais, e pouco foi desenvolvido para a análise de dados BS correlacionados. Podemos citar Villegas et al. (2011) que estudaram modelos lineares mistos com erros log-BS e, mais recentemente, Kundu et al. (2013) derivaram a versão multivariada da distribuição BS. Marchant et al. (2016a) derivaram modelos log-lineares para a distribuição BS enquanto Marchant et al. (2016b) apresentaram procedimentos de diagnóstico em modelos de regressão BS multivariados generalizados.

A independência entre as observações é uma das principais suposições dos modelos de regressão tradicionais tais como os modelos lineares generalizados (MLGs). Contudo, é comum nos depararmos com dados correlacionados, como ocorre em dados agrupados ou entre observações realizadas em um mesmo indivíduo ao longo do tempo, comum em estudos longitudinais. Diante disso, Wedderburn (1974) introduziu modelos de quase-verossimilhança que estendem a ideia dos MLGs para situações mais gerais incluindo dados correlacionados. Liang e Zeger (1986) e Zeger e Liang (1986) propuseram o método de equações de estimação generalizadas (EEGs) com o objetivo de produzir estimativas eficientes e não viesadas para os parâmetros do modelo de regressão quando se lida com dados correlacionados não gaussianos. Nesses estudos é interessante o fato que a matriz de correlação de trabalho especificada para o vetor de medidas repetidas de cada unidade experimental não precisa estar corretamente especificada para se obter consistência dos parâmetros de regressão. Prentice e Zhao (1991) estenderam a proposta de Liang e Zeger (1986), que ficou conhecida como EEG1, e estimando também os parâmetros de correlação via equações de estimação, desenvolvendo o que é denominado na literatura como EEG2. Mais tarde surgiram propostas em que equações de estimação também passaram a ser utilizadas para modelar o parâmetro de dispersão (EEG3), veja por exemplo Song et al. (2004). O método de equações de estimação generalizadas tornou-se uma ferramenta importante para a análise de dados correlacionados. Por exemplo, Horton et al. (1999) ajustaram um modelo de regressão logístico marginal utilizando equações de estimação generalizadas para dados de medidas repetidas em um estudo sobre utilização de serviços de saúde mental. Artes (1997) estudou equações de estimação para dados longitudinais circulares e modelos de dispersão e Venezuela (2008) propôs equações de estimação generalizadas para modelos de regressão beta com medidas repetidas. Alencar et al. (2012), motivados por um exemplo prático envolvendo dados longitudinais pré-teste e pós-teste, traçaram uma comparação considerando modelos lineares log-normais mistos, modelos lineares generalizados mistos e modelos baseados em equações de estimação generalizadas. Os autores concluiram que modelos baseados em EEG podem ser preferíveis quando o objetivo é comparar as respostas esperadas marginais.

Assim, propomos neste trabalho a modelagem de dados correlacionados usando as distribuições BS e BSG sob o ponto de vista de equações de estimação generalizadas. 


\subsection{Considerações preliminares}

Nesta seção faremos uma revisão da literatura que norteou os estudos necessários para o desenvolvimento desse trabalho. Serão inicialmente apresentadas as distribuições: BS, log-BS, BSG, em particular a BS- $t$, e a log-BS-t. Será discutido então o modelo de regressão-log-BS, introduzido por Rieck e Nedelman (1991) e amplamente utilizado. Em seguida realizaremos uma breve explanação sobre métodos que vêm sendo utilizados com o intuito de analisar dados BS correlacionados. Por fim, segue uma revisão dos textos introdutórios referentes a equações de estimação, baseados em Liang e Zeger (1986), Godambe (1997), Ziegler (2008) e Artes e Botter (2005).

\subsubsection{Distribuição Birnbaum-Saunders}

A distribuição Birnbaum-Saunders (BS) é uma distribuição de vida (life distribution) que relaciona o tempo até a ocorrência de falha com algum dano cumulativo que é assumido gaussiano. Conhecida também como distribuição de vida por fadiga, vem sendo amplamente aplicada em diversas áreas do conhecimento, tais como engenharia, medicina, contaminação atmosférica, qualidade da água, catástrofes naturais, negócios, finanças, indústria, ciência de gestão e controle de qualidade. A principal vantagem das pesquisas relacionadas a essa distribuição é o melhor ajuste nos percentis mais baixos ou mais altos da distribuição (onde há o maior interesse dos analistas) quando comparada às demais distribuições de vida. Mais detalhes, ver Birnbaum e Saunders (1969) e Leiva (2016).

A fadiga é um dano estrutural que ocorre quando um material é exposto a flutuações de estresse e tensão. Birnbaum e Saunders (1969) construíram essa família de distribuições de vida baseada em um determinado tipo de fadiga. Vejamos a seguir como foi obtida essa distribuição.

O processo de fadiga de materiais consiste em três etapas. A primeira etapa trata-se de uma fissura imperceptível, referente aos percentis 5 e 10 da vida útil do material. A segunda etapa é o crescimento da fissura, gerado pelo estresse cíclico e tensão. A terceira etapa é por fim a ruptura ou falha do material devido à fadiga. Essa etapa trata-se de um evento pontual.

Durante um processo de fadiga o material é exposto a um padrão cíclico de tensão e força. Isto é, se $l_{1}, l_{2}, \ldots, l_{m}$ é a sequência de $m$ cargas aplicadas em um ciclo, essa mesma sequência será aplicada em cada ciclo de cargas. Formalmente, para algum $m>1$, para quaisquer dois ciclos $j$ e $k$ e para todo $i=1, \ldots, m$, temos

$$
\ell_{j m+i}=\ell_{k m+i}
$$

Essas cargas geram o desgaste do material causando uma fissura. Conforme o processo acontece ocorre o crescimento de uma fissura dominante. A falha ocorre quando a fissura ultrapassa um certo nível de resistência, denominado valor crítico e denotado por $\omega$.

Sejam as variáveis aleatórias:

1. $\mathrm{x}_{i}$ que denota a extensão incremental da fissura na $i$-ésima oscilação de carga.

2. $\mathrm{y}_{j+1}=\mathrm{x}_{j m+1}+\ldots+\mathrm{x}_{j m+m}$ que corresponde à extensão da fissura durante o $(j+1)$-ésimo ciclo.

Birnbaum e Saunders (1969) então fizeram as seguintes suposições: 
(I) $\mathrm{x}_{i}$ é uma variável aleatória com uma distribuição que só depende da fissura atual causada pela tensão neste ciclo.

(II) A extensão total da fissura, $\mathrm{y}_{j}$, devido ao $j$-ésimo ciclo é uma variável aleatória que segue uma distribuição com média $\mu$ e variância $\sigma^{2}, \forall j=1,2, \ldots$

Logo, da suposição (I) segue a independência das variáveis aleatórias $\left(\mathrm{y}_{1}, \mathrm{y}_{2}, \ldots\right)$ e da suposição (II) o fato delas serem identicamente distribuídas. Ao serem aplicados $n$ ciclos de cargas, a extensão total da fissura será a fissura acumulada durante todos os $n$ ciclos, e será dada pela variável aleatória,

$$
\mathrm{w}_{n}=\sum_{j=1}^{n} \mathrm{y}_{j}
$$

com função de distribuição,

$$
H_{n}(w)=P\left(\mathrm{w}_{n} \leq w\right)
$$

Seja $N$ o número de ciclos de cargas necessários para que ocorra a ruptura do material. Em outras palavras, $N$ é o número de ciclos que devem ocorrer para que a extensão total da fissura ultrapasse o valor crítico $\omega$. Determinamos a função de distribuição de $N$ por,

$$
\begin{aligned}
P(N \leq n) & =P\left(\sum_{j=1}^{n} \mathrm{y}_{j}>\omega\right)=1-P\left(\sum_{j=1}^{n} \mathrm{y}_{j} \leq \omega\right) \\
& =1-P\left(\frac{\sum_{j=1}^{n}\left(\mathrm{y}_{j}-\mu\right)}{\sigma \sqrt{n}} \leq \frac{\omega-n \mu}{\sigma \sqrt{n}}\right) \\
& =1-P\left(\frac{\sum_{j=1}^{n}\left(\mathrm{y}_{j}-\mu\right)}{\sigma \sqrt{n}} \leq \frac{\omega}{\sigma \sqrt{n}}-\frac{\sqrt{n} \mu}{\sigma}\right) .
\end{aligned}
$$

Logo, do Teorema do Limite Central, para variáveis aleatórias independentes e identicamente distribuídas, segue que

$$
\begin{aligned}
P(N \leq n) & \cong 1-\Phi\left(\frac{\omega}{\sigma \sqrt{n}}-\frac{\sqrt{n} \mu}{\sigma}\right)=\Phi\left[-\left(\frac{\omega}{\sigma \sqrt{n}}-\frac{\sqrt{n} \mu}{\sigma}\right)\right] \\
& =\Phi\left(\frac{\sqrt{n} \mu}{\sigma}-\frac{\omega}{\sigma \sqrt{n}}\right)
\end{aligned}
$$

em que $\Phi($.$) é a função de distribuição acumulada da distribuição normal padrão.$

Birnbaum e Saunders (1969) então propuseram substituir $n$ em (1.2) por uma variável real não negativa t. Dessa forma, segundo os autores, t é a extensão contínua da variável discreta $N$, em que t é o tempo total até que ocorra a falha.

A função de distribuição acumulada da variável aleatória t fica dada por:

$$
F(t ; \beta, \alpha)=P(\mathrm{t} \leq t)=\Phi\left[\frac{1}{\alpha}\left(\sqrt{\frac{t}{\beta}}-\sqrt{\frac{\beta}{t}}\right)\right], t>0,
$$

com, $\alpha=\frac{\sigma}{\sqrt{\omega \mu}}>0$ e $\beta=\frac{\omega}{\mu}>0$. E assim dizemos que t segue uma distribuição BS com parâmetros 
$\alpha$ e $\beta$ e denotamos por $\mathrm{t} \sim B S(\alpha, \beta)$.

Podemos obter a variável aleatória $\mathrm{t} \sim B S(\alpha, \beta)$ a partir da distribuição normal da seguinte forma:

$$
\mathrm{t}=\beta\left[\frac{\alpha \mathrm{z}}{2}+\sqrt{\left(\frac{\alpha \mathrm{z}}{2}\right)^{2}+1}\right]^{2}
$$

em que $\mathrm{z} \sim N(0,1)$. Mais detalhes ver, por exemplo, Díaz-García e Leiva (2005).

A função densidade de probabilidade de uma variável aleatória $\mathrm{t} \sim B S(\alpha, \beta)$ é obtida derivando (1.3) em relação a $t$. De modo que,

$$
f(t ; \alpha, \beta)=\frac{1}{2 \alpha \sqrt{2 \pi \beta}}\left[\left(\frac{\beta}{t}\right)^{\frac{1}{2}}+\left(\frac{\beta}{t}\right)^{\frac{3}{2}}\right] \exp \left[-\frac{1}{2 \alpha^{2}}\left(\frac{t}{\beta}+\frac{\beta}{t}-2\right)\right] .
$$

A média e a variância existem e são dadas, respectivamente por,

$$
\mathrm{E}(\mathrm{t})=\beta\left(1+\frac{\alpha^{2}}{2}\right) \text { e } \operatorname{Var}(\mathrm{t})=(\alpha \beta)^{2}\left(1+\frac{5}{4} \alpha^{2}\right)
$$

\subsubsection{Distribuição log-Birnbaum-Saunders}

A partir do momento em que a distribuição normal foi criada passou a ser amplamente aplicada e desenvolvida. Johnson (1949) usou o método de translação, introduzido por Edgeworth (1898), para gerar famílias de distribuições a partir de transformações da forma $\mathrm{z}=\nu+\delta f(\mathrm{y} ; \mu, \sigma)$, em que z é uma variável normal padrão, $f(y ; \mu, \sigma)$ é uma função monótona simples, $\nu$ e $\delta$ são parâmetros de forma, $\sigma$ é parâmetro de escala e $\mu$ é parâmetro de posição. Rieck e Nedelman (1991) desenvolveram a distribuição senh-normal (SN) através da transformação y $=\operatorname{arcsenh}(\alpha \mathrm{z} / 2) \sigma+\mu$, em que z tem distribuição normal padrão, $\alpha>0$ é o parâmetro de forma, $\mu \in \mathbb{R}$ é o parâmetro de localização e $\sigma>0$ é o parâmetro de escala. Denotamos então y $\sim \operatorname{SN}(\mu, \alpha, \sigma)$, e sua função densidade de probabilidade fica dada por:

$$
f(y)=\frac{2}{\alpha \sigma \sqrt{2 \pi}} \cosh \left(\frac{y-\mu}{\sigma}\right) \exp \left\{-\frac{1}{2}\left[\frac{2}{\alpha} \operatorname{senh}\left(\frac{y-\mu}{\sigma}\right)\right]^{2}\right\}, y \in \mathbb{R} .
$$

Ainda podemos dizer que se $\mathrm{y} \sim \mathrm{SN}(\alpha, \mu, \sigma)$ então $\mathrm{z}=\frac{2}{\alpha} \operatorname{Senh}\left[\frac{(\mathrm{y}-\mu)}{\sigma}\right] \sim N(0,1)$.

A função de distribuição acumulada da variável aleatória y é então dada por

$$
F_{\mathrm{y}}(y)=\Phi\left\{\frac{2}{\alpha} \operatorname{senh}\left(\frac{y-\mu}{\sigma}\right)\right\}, y \in \mathbb{R}
$$

em que $\Phi(\cdot)$ é a distribuição acumulada da distribuição normal padrão. Quanto aos parâmetros dessa distribuição sabemos que o parâmetro $\alpha$ influencia a curtose, de modo que a curva da distribuição torna-se mais achatada à medida que o valor de $\alpha$ aumenta e $\sigma$ é o parâmetro de escala, de modo que a amplitude da curva diminui à medida que $\sigma$ aumenta. 
A função geradora de momentos para a distribuição SN é dada por

$$
m(t)=\exp (\mu s)\left[\frac{K_{a}\left(\alpha^{-2}\right)+K_{b}\left(\alpha^{-2}\right)}{2 K_{1 / 2}\left(\alpha^{-2}\right)}\right]
$$

em que $a=(\sigma s+1) / 2, b=(\sigma s-1) / 2$ e $K(\cdot)$ é a função de Bessel modificada do terceiro tipo, dada por

$$
K_{\lambda}(w)=\frac{1}{2}\left(\frac{w}{2}\right)^{\lambda} \int_{0}^{\infty} y^{-\lambda-1} e^{-y-\left(w^{2} / 4 y\right)} d y .
$$

De (1.8) podemos obter a esperança e a variância da variável aleatória y $\sim \operatorname{SN}(\alpha, \mu, \sigma)$ dadas, respectivamente, por

$$
\mathrm{E}(\mathrm{y})=\mu \quad \text { e } \quad \operatorname{Var}(\mathrm{y})=\sigma^{2} g(\alpha)
$$

em que $g(\alpha)$ é uma função baseada em $K_{\lambda}(\cdot)=K_{-\lambda}(w)$ que depende apenas de $\alpha$. Mais detalhes ver Villegas et al. (2011).

Uma importante relação entre as distribuições BS e SN foi mostrada por Rieck (1989). Se a variável aleatória t tem distribuição $\mathrm{BS}(\alpha, \beta)$, então $\mathrm{y}=\log (\mathrm{t})$ tem distribuição $\operatorname{SN}(\alpha, \mu, 2)$, com $\mu=\log (\beta)$. Logo, a distribuição $\log$-BS é um caso particular da distribuição SN (senh-normal). Essa distribuição é unimodal para $\alpha<2$, platicúrtica para $\alpha=2$ e bimodal para $\alpha>2$.

A função densidade de probabilidade de y pode ser expressa como

$$
f(y ; \alpha, \mu)=\frac{1}{\alpha \sqrt{2 \pi}} \cosh \left(\frac{y-\mu}{2}\right) \exp \left[-2\left\{\frac{1}{\alpha} \operatorname{senh}\left(\frac{y-\mu}{2}\right)\right\}^{2}\right] .
$$

\subsubsection{Distribuição Birnbaum-Saunders generalizada}

De acordo com Díaz-García e Leiva (2005), qualquer análise estatística em que uma distribuição normal é assumida pode ser generalizada para a família de distribuições elípticas. Segundo os autores, apesar de não haver argumentos nem empíricos e nem relacionados às leis da física, existem razões puramente estatísticas ou matemáticas, no sentido de que a teoria desenvolvida sob distribuição normal é um caso particular da teoria obtida sob distribuições elípticas. Muitas das propriedades de uma distribuição normal são extensíveis ao caso de distribuições elípticas e algumas importantes estatísticas na teoria da inferência normal são invariantes sob toda a família de distribuições elípticas.

Diante disso, Díaz-García e Leiva (2005) apresentaram uma generalização da distribuição BS, que foi derivada com base em distribuições de contornos elípticos e foi denominada de distribuição Birnbaum-Saunders generalizada (BSG).

Definição 1.1 Uma variável aleatória x segue distribuição elíptica se sua função característica é dada por,

$$
\varphi_{\mathbf{x}}(t)=\exp (i t \mu) \phi\left(t^{2} \sigma^{2}\right)
$$


com $\phi: \mathbb{R} \rightarrow \mathbb{R}^{+}$, ou se a fdp de x é dada por,

$$
f_{x}(x)=c g\left[\frac{(x-\mu)^{2}}{\sigma^{2}}\right]=c g(u) ; x \in \mathbb{R}, u>0,
$$

em que $g(u)$ é o núcleo da $f d p$ de $x$ e c é a constante normalizadora que faz com que $f_{x}$ seja uma função densidade de probabilidade. A notação é dada por $\mathrm{x} \sim \operatorname{EC}\left(\mu, \sigma^{2} ; \phi\right)$, quando é usada a forma (1.11) e $\mathrm{x} \sim \operatorname{EC}\left(\mu, \sigma^{2} ; \mathrm{g}\right)$ se, a forma usada é (1.12).

Comumente, $\mu$ é o parâmetro de posição, que coincide com a média, $\mathrm{E}(\mathrm{x})$, se o primeiro momento da distribuição existe e $\sigma^{2}$ é o parâmetro de escala. Para casos unidimensionais de variáveis aleatórias as distribuições elípticas correspondem a todas as distribuições simétricas em $\mathbb{R}$.

O modelo desenvolvido por Díaz-García e Leiva (2005) foi obtido relaxando a suposição de normalidade de (1.4). De modo que os autores passaram a considerar,

$$
\mathrm{t}=\beta\left[\frac{\alpha U}{2}+\sqrt{\left(\frac{\alpha U}{2}\right)^{2}+1}\right]^{2},
$$

em que $U \sim E C\left(\mu, \sigma^{2} ; g\right)$. E então, a variável aelatória t segue distribuição BSG e é denotada por, $\mathrm{t} \sim B S G(\alpha, \beta ; g)$, em que $\alpha$ é o parâmetro de forma, $\beta$ é o parâmetro de escala e $g(\cdot)$ determina o núcleo da função densidade e é dada em (1.12). A motivação do uso dessa nova família de distribuições reside no fato dela ser mais flexível por possuir caudas mais ou menos pesadas do que a distribuição BS já estudada.

Seja t $\sim B S G(\alpha, \beta ; g)$, então a função densidade de probabilidade é dada por,

$$
f_{\mathrm{t}}(t)=\frac{c}{2 \alpha \beta^{1 / 2}} t^{3 / 2}(t+\beta) g\left[\frac{1}{\alpha^{2}}\left(\frac{t}{\beta}+\frac{\beta}{t}-2\right)\right], t>0 .
$$

Díaz-García e Leiva (2005) consideraram diferentes núcleos para a fdp de t. Como por exemplo: função t-Student, normal (que recai na função de distribuição BS clássica), Cauchy, Kotz, logística, entre outras.

Trabalharemos apenas com a distribuição BSG obtida a partir da distribuição t-Student. A função densidade de probabilidade, neste caso, fica dada por,

$$
f_{\mathrm{t}}(t)=\frac{\Gamma((\nu+1) / 2) t^{-3 / 2}(t+\beta)}{2 \alpha(\nu \pi \beta)^{1 / 2} \Gamma(\nu / 2)}\left(1+\frac{1}{\nu \alpha^{2}}\left[\frac{t}{\beta}+\frac{\beta}{t}-2\right]\right)^{-(\nu+1) / 2},
$$

em que $\alpha, \beta, \nu>0$. E diremos que $\mathrm{t} \sim \operatorname{BS}-t(\alpha, \beta, \nu)$.

\subsubsection{Distribuição log-BS- $t$}

Por analogia ao caso da distribuição SN, Díaz-García e Domínguez-Molina (2006) obtiveram a distribuição seno hiperbólica esférica (senh-esférica), denotada por $S S(\alpha, \mu, \sigma ; g)$, cuja densidade é dada por

$$
f(y)=\frac{\mathrm{c}}{\sigma}\left\{\frac{2}{\alpha} \cosh \left(\frac{y-\mu}{2}\right)\right\} g\left[\left(\frac{2}{\alpha} \operatorname{senh}\left(\frac{y-\mu}{2}\right)\right)\right]^{2} .
$$


Se $\mathrm{t} \sim \operatorname{BS}-t(\alpha, \beta, \nu)$ então $\mathrm{y}=\log (\mathrm{t}) \sim \mathrm{SS}(\alpha, \mu, 2, \nu)$, ou distribuição $\log$-BS- $t(\alpha, \mu, \nu)$. Seja $g(\cdot)$ o núcleo da distribuição $t$-Student, ou seja, $g(u)=\left\{1+\frac{1}{\nu} u\right\}^{-\left(\frac{\nu+1}{2}\right)}$ e c $=\mathrm{c}(\nu)=\frac{\Gamma\left(\frac{\nu+1}{2}\right)}{(\nu \pi)^{1 / 2} \Gamma\left(\frac{\nu}{2}\right)}$, em que $\Gamma(t)=\int_{0}^{\infty} x^{t-1} \mathrm{e}^{-x} d x$. A função densidade de probabilidade de y fica dada por

$$
f(y)=\mathrm{c}(\nu)\left\{\frac{1}{\alpha} \cosh \left(\frac{y-\mu}{2}\right)\right\}\left\{1+\frac{1}{\nu}\left[\frac{2}{\alpha} \operatorname{senh}\left(\frac{y-\mu}{2}\right)\right]^{2}\right\}^{-\left(\frac{\nu+1}{2}\right)} .
$$

\subsubsection{Modelo de regressão log-Birnbaum-Saunders}

Rieck e Nedelman (1991) motivados pela importância de prever a performance de materiais sob diferentes condições, desenvolveram um modelo log-linear para a distribuição BS, baseado na distribuição SN. Antes do desenvolvimento desse modelo análises estatísticas referentes à distribuição BS tinham sido limitadas aos casos em que são consideradas amostras únicas. Apresentaremos a seguir o modelo desenvolvido pelos autores.

Sejam $\mathrm{t}_{1}, \mathrm{t}_{2}, \ldots, \mathrm{t}_{n}$ variáveis aleatórias independentes tais que $\mathrm{t}_{i} \sim \operatorname{BS}\left(\alpha, \theta_{i}\right)$, para $i=1, \ldots, n$. Supondo que a distribuição de cada $\mathrm{t}_{i}$ depende de $\mathbf{x}_{i}=\left(x_{i 1}, x_{i 2}, \ldots, x_{i p}\right)^{\top}$, um conjunto de valores de variáveis explicativas $(p<n)$, e assumindo que:

i) $\theta_{i}=\exp \left\{\mathbf{x}_{i}^{\top} \boldsymbol{\beta}\right\}$, em que $\boldsymbol{\beta}=\left(\beta_{1}, \beta_{2}, \ldots, \beta_{p}\right)^{\top}$ é o vetor de parâmetros desconhecidos a ser estimado e,

ii) o parâmetro de forma é independente das variáveis explicativas.

então, $\mathrm{t}_{i} \sim \mathrm{BS}\left(\alpha, \exp \left\{\mathbf{x}_{i}^{\top} \boldsymbol{\beta}\right\}\right)$.

Como a distribuição BS é fechada sob transformações de escala, considerando que $\epsilon_{i}^{*} \sim \operatorname{BS}(\alpha, 1)$, então, $\mathrm{t}_{i}=\exp \left\{\mathbf{x}_{i}^{\top} \boldsymbol{\beta}\right\} \epsilon_{i}^{*} \sim \mathrm{BS}\left(\alpha, \exp \left\{\mathbf{x}_{i}^{\top} \boldsymbol{\beta}\right\}\right)$. Portanto, fazendo $\mathrm{y}_{i}=\log \left(\mathrm{t}_{i}\right)$ obtém-se o modelo de regressão log-linear BS

$$
\mathrm{y}_{i}=\mathbf{x}_{i}^{\top} \boldsymbol{\beta}+\epsilon_{i},
$$

em que $\mathrm{y}_{i}$ é o logaritmo do tempo para a $i$-ésima unidade experimental, $\boldsymbol{\beta}=\left(\beta_{1}, \beta_{2}, \ldots, \beta_{p}\right)^{\top}$ é o vetor de parâmetros desconhecidos a ser estimado, $\mathbf{x}_{i}=\left(x_{i 1}, x_{i 2}, \ldots, x_{i p}\right)^{\top}$ são valores de variáveis explicativas e $\epsilon_{i}=\log \left(\epsilon_{i}^{*}\right)$ são variáveis aleatórias independentes e identicamente distribuídas tais que $\epsilon_{i} \sim \mathrm{SN}(\alpha, 0,2)$.

O modelo pode ser usado para teste de vida acelerada ou para comparar vidas medianas de várias populações. Rieck e Nedelman (1991) apresentaram ainda a inferência para os parâmetros desconhecidos, discutiram métodos de estimação pontual, teste de hipóteses e intervalos de confiança. Galea et al. (2004) apresentaram métodos de diagnóstico para um modelo de regressão log-linear com distribuição BS para os erros. Os autores desenvolveram as medidas de diagnóstico: influência local, influência local total e alavanca generalizada. Leiva et al. (2007) trabalharam com métodos de diagnóstico no modelo de regressão log-BS com dados censurados. Barros et al. (2008) apresentaram um modelo baseado na BS- $t$. Discutiram procedimentos de estimação robusta e métodos de diagnóstico. Os autores motivaram o estudo justificando que a distribuição BS clássica é sensível a observações aberrantes e a BS- $t$ surge como uma alternativa a esse modelo. Lemonte e Cordeiro 
(2009) introduziram uma classe de modelos de regressão não lineares BS, que trata de uma generalização do modelo de regressão desenvolvido por Rieck e Nedelman (1991). Lemonte e Patriota (2011)) generalizaram então os métodos de diagnóstico de Galea et al. (2004) para a classe de modelos desenvolvida em Lemonte e Cordeiro (2009).

\subsubsection{Modelos mistos}

Villegas et al. (2011), partindo do fato que a maioria dos modelos baseados na distribuição BS, supõe efeitos fixos e que pouco tem sido investigado para dados correlacionados, introduziram os modelos BS mistos para dados censurados.

Para motivar o trabalho os autores consideram um exemplo onde uma amostra de máquinas é selecionada aleatoriamente. Oe estimando tamb é estabelecer uma relação entre o desempenho da máquina (variável resposta) e o nível de estresse (preditor). Cada máquina tem seu desempenho avaliados em níveis diferentes de estresse. Assim, devido à possível correlação entre as medidas de desempenho de cada máquina, um modelo de efeito aleatório mostra-se mais apropriado do que um modelo de efeitos fixos.

Seja $\mathrm{y}_{i j}$ o $j$-ésimo logaritmo da resposta associada ao $i$-ésimo grupo (indivíduo), para $i=1, \ldots, n$ e $j=1, \ldots, s_{i}$. O modelo log-BS de efeitos aleatórios, apresentado por Villegas et al. (2011), segue a seguinte estrutura hierárquica:

$$
\begin{aligned}
\mathrm{y}_{i j} \mid b_{i} & \sim \log -\mathrm{BS}\left(\alpha, \mu_{i j}\right) \mathrm{e} \\
\mathbf{b}_{i} & \sim \mathrm{N}_{d}(0, \boldsymbol{\zeta})
\end{aligned}
$$

e componente sistemática

$$
\mu_{i j}=\mathrm{E}\left(\mathrm{y}_{i j} \mid b_{i}\right)=\mathbf{x}_{i j}^{\top} \boldsymbol{\beta}+\mathbf{z}_{i j}^{\top} \mathbf{b} i
$$

em que $i=1, \ldots, n, j=1, \ldots, s_{i}, \mathrm{y}_{i j}$ é a variável resposta e corresponde ao logaritmo do tempo de falha ou censura, $\mathbf{x}_{i j}=\left(x_{i j 1}, \ldots, x_{i j p}\right)^{\top}$ contém valores de variáveis explicativas associadas aos efeitos fixos, $\boldsymbol{\beta}=\left(\beta_{1}, \ldots, \beta_{p}\right)^{\top}$ é o vetor de efeitos fixos que precisa ser estimado, $z_{i j}=\left(z_{i j 1}, \ldots, z_{i j q}\right)^{\top}$ contém valores de variáveis explicativas associadas aos efeitos aleatórios, $\mathbf{b}_{i}=\left(b_{i 1}, \ldots, b_{i q}\right)^{\top}$ é um vetor de efeitos aleatórios, e $\boldsymbol{\zeta}$ é a matriz de variância-covariância $q \times q$ dos efeitos aleatórios. Tem-se que,

$$
\begin{aligned}
\mathrm{E}\left(\mathrm{y}_{i j}\right) & =\mathbf{x}_{i j}^{\top} \boldsymbol{\beta}, \\
\operatorname{Var}\left(\mathrm{y}_{i j}\right) & =4 K(\alpha)+\mathbf{z}_{i j}^{\top} \boldsymbol{\zeta} \mathbf{z}_{i j} \mathrm{e} \\
\operatorname{Cov}\left(\mathrm{y}_{i j}, \mathrm{y}_{i l}\right) & =\mathbf{z}_{i j}^{\top} \boldsymbol{\zeta} \mathbf{z}_{i l} .
\end{aligned}
$$

Então, a correlação intraclasse é dada por

$$
\operatorname{Corr}\left(\mathrm{y}_{i j}, \mathrm{y}_{i l}\right)=\frac{\mathbf{z}_{i j}^{\top} \boldsymbol{\zeta} \mathbf{z}_{i l}}{4 K(\alpha)+\mathbf{z}_{i j}^{\top} \boldsymbol{\zeta} \mathbf{z}_{i l}},
$$

para $i=1, \ldots, n, j=1, \ldots, s_{i}$ e $j \neq l$. 


\subsubsection{Distribuição Birnbaum-Saunders multivariada generalizada}

Kundu et al. (2010) introduziram a distribuição BS bivariada com cinco parâmetros, obtida a partir de uma transformação monótona de duas variáveis com distribuição normal bivariada. Em Kundu et al. (2013), foi desenvolvida a distribuição BS multivariada generalizada baseada na distribuição simétrica elíptica multivariada no lugar da distribuição normal multivariada.

Marchant et al. (2016a) e Marchant et al. (2016b) derivaram o modelo de regressão log-linear GBS multivariado e apresentaram procedimentos de diagnósticos para esses modelos, respectivamente.

Um vetor aleatório $\mathbf{X}, p$-dimensional, é dito ter distribuição elíptica simétrica com vetor de localização $\boldsymbol{\mu}, p \times 1$, matriz de dispersão $p \times p, \boldsymbol{\Sigma}$, e gerador de desnsidade $h^{(p)}(\cdot)$, se a função densidade de probabilidade de $\mathbf{X}$ é da forma,

$$
f\left(\mathbf{x}, \boldsymbol{\mu}, \boldsymbol{\Sigma}, h^{(p)}\right)=c_{p}|\boldsymbol{\Sigma}|^{-1 / 2} h^{(p)}(w(\mathbf{x})),
$$

com $\mathbf{x} \in \mathbb{R}^{p}, w(\mathbf{x}): \mathbb{R}^{p} \longrightarrow \mathbb{R}_{+}$tal que $w(\mathbf{x})=(\mathbf{x}-\boldsymbol{\mu})^{\top} \boldsymbol{\Sigma}^{-1}(\mathbf{x}-\boldsymbol{\mu}), h^{(p)}: \mathbb{R}_{+} \longrightarrow \mathbb{R}_{+}, c_{p}>0 \mathrm{e}$

$$
\int_{\mathbb{R}^{p}} f\left(\mathbf{x}, \boldsymbol{\mu}, \boldsymbol{\Sigma}, h^{(p)}\right) d \mathbf{x}=1
$$

A possibilidade de escolher a função geradora $h^{(p)}(\cdot)$, permite diferentes distribuições simétricas elípticas. As distribuições normal multivariada e t-multivariada (com graus de liberdade $\nu>0$ ) são de grande interesse prático.

A função densidade de probabilidade da distribuição normal multivariada é dada por

$$
f(\mathbf{x}, \boldsymbol{\mu}, \boldsymbol{\Sigma})=(2 \pi)^{-p / 2}|\boldsymbol{\Sigma}|^{-1 / 2} \exp \left\{-\frac{1}{2}(\mathbf{x}-\boldsymbol{\mu})^{\top} \boldsymbol{\Sigma}^{-1}(\mathbf{x}-\boldsymbol{\mu})\right\},
$$

e tomando $c_{p}=(2 \pi)^{-p / 2}, h^{(p)}(w(\mathbf{x}))=\exp -w(\mathbf{x}) / 2$ reduz-se á forma dada em (1.17).

Definição 1.2 Sejam $\boldsymbol{\alpha}$ e $\boldsymbol{\beta} \in \mathbb{R}^{p}$, com $\boldsymbol{\alpha}=\left(\alpha_{1}, \ldots, \alpha_{p}\right)^{\top}$ e $\boldsymbol{\beta}=\left(\beta_{1}, \ldots, \beta_{p}\right)^{\top}$, com $\alpha_{i}>0$. $\beta_{i}>0$, para $i=1, \ldots, p$. Seja $\boldsymbol{\Gamma} p \times p$ matriz de correlação positiva definida. Então, o vetor aleatório $\mathbf{t}=\left(t_{1}, \ldots, t_{p}\right)^{\top}$ é dito ter distribuição $B S$ multivariada generalizada com parâmetros $(\boldsymbol{\alpha}, \boldsymbol{\beta}, \boldsymbol{\Gamma})$ e função geradora $h^{(p)}$, denotado por $\mathbf{t} \sim G B S_{p}\left(\boldsymbol{\alpha}, \boldsymbol{\beta}, \boldsymbol{\Gamma}, h^{(p)}\right)$, se a função de distribuição acumulada é dada por,

$$
P(\mathbf{t} \leq \mathbf{t})=F\left[\frac{1}{\alpha_{1}}\left(\sqrt{\frac{t_{1}}{\beta_{1}}}-\sqrt{\frac{\beta_{1}}{t_{1}}}\right), \ldots, \frac{1}{\alpha_{p}}\left(\sqrt{\frac{t_{p}}{\beta_{p}}}-\sqrt{\frac{\beta_{p}}{t_{p}}}\right) ; \boldsymbol{\Gamma}, h^{(p)}\right]
$$

para $\mathbf{t}>0$ e $F\left(; \boldsymbol{\Gamma}, h^{(p)}\right)$ denota a função de distribuição acumulada do vetor aleatório com distribuição simétrica elíptica com $\mathbf{\Sigma}=\boldsymbol{\Gamma}$ e $h^{(p)}$ a função geradora. A função densidade de probabilidade de $\mathbf{t}$ é dada por,

$$
f(\mathbf{t}, \boldsymbol{\alpha}, \boldsymbol{\beta}, \boldsymbol{\Gamma})=f\left[\frac{1}{\alpha_{1}}\left(\sqrt{\frac{t_{1}}{\beta_{1}}}-\sqrt{\frac{\beta_{1}}{t_{1}}}\right), \ldots, \frac{1}{\alpha_{p}}\left(\sqrt{\frac{t_{p}}{\beta_{p}}}-\sqrt{\frac{\beta_{p}}{t_{p}}}\right) ; \boldsymbol{\Gamma}, h^{(p)}\right]
$$




$$
\times \prod_{i=1}^{p}\left\{\left(\frac{\beta_{i}}{t_{i}}\right)^{1 / 2}+\left(\frac{\beta_{i}}{t_{i}}\right)^{3 / 2}\right\}
$$

e $f(\cdot)$ é dado em (1.17).

\subsubsection{Funções de estimação}

O método de equações de estimação generalizadas (EEGs) foi proposto por Liang e Zeger (1986) e recebeu considerável atenção em pesquisas estatísticas. Para mais detalhes, indicamos Ziegler (2008) e Godambe (1997), onde são revisados pontos importantes no desenvolvimento desse método e algumas aplicações são apresentadas.

Segundo Godambe (1997) existem três importantes precursores na teoria moderna de funções de estimação: o método de mínimos quadrados proposto por Legendre (1805), o método dos momentos proposto por Pearson em algum instante do último século e o método de máxima verossimilhança proposto por Fisher (1925).

Vamos agora introduzir alguns conceitos importantes à respeito do estudo de funções de estimação.

Como definido em Artes et al. (2000) funções de estimação são funções mensuráveis de dados e parâmetros de interesse, cujas raízes resultantes das equações de estimação são as estimatvas dos parâmetros.

Definição 1.3 Seja $\chi \in \mathbb{R}$ um espaço amostral sobre o qual define-se uma família $\wp=\left\{P_{\boldsymbol{\theta}}, \boldsymbol{\theta} \in\right.$ $\left.\Theta \subseteq \mathbb{R}^{p}\right\}$ de distribuições de probabilidade indexadas por um parâmetro $\boldsymbol{\theta}$ desconhecido. Por definição, uma função $\boldsymbol{\psi}: X \times \Theta \rightarrow \mathbb{R}$, é uma função de estimação, se para cada $\boldsymbol{\theta} \in \Theta, \boldsymbol{\psi}(., \boldsymbol{\theta})$ é uma variável aleatória.

Artes (1997) ressalta que é desejável que uma pequena variação no vetor paramétrico acarrete uma grande variação no valor da função de estimação, pois quanto maior essa variação, mais eficiente a função será na estimação do parâmetro.

Assumimos a existência de uma amostra de $n$ vetores aleatórios independentes $\mathbf{y}_{\mathbf{1}}, \ldots, \mathbf{y}_{\mathbf{n}}$, tal que $\mathbf{y}_{\mathbf{i}}=\left(y_{i 1}, \ldots, y_{i s_{i}}\right)^{\top}$. Cada unidade amostral será associada a uma função de estimação que será denotada por, $\boldsymbol{\psi}_{i}$. De forma que a função de estimação para a amostra é dada por, $\boldsymbol{\Psi}_{n}(\mathbf{y}, \boldsymbol{\theta})=\sum_{i=1}^{n} \boldsymbol{\psi}_{i}\left(\mathbf{y}_{\mathbf{i}}, \boldsymbol{\theta}\right)$.

Vamos nos deter ao estudo das funções de estimação cujas raízes são estimadores dos parâmetros de interesse, ou seja,

$$
\mathbf{\Psi}_{n}\left(\mathbf{y}, \hat{\boldsymbol{\theta}}_{n}\right)=\mathbf{0}
$$

Essas equações são denominadas equações de estimação. Para simplificar a notação usaremos $\boldsymbol{\Psi}_{n}\left(\boldsymbol{\theta}_{\boldsymbol{n}}\right)$ no lugar de $\boldsymbol{\Psi}_{n}\left(\mathbf{y}, \boldsymbol{\theta}_{\boldsymbol{n}}\right)$ (desde que não atrapalhe o entendimento do texto.)

A função de estimação $\boldsymbol{\Psi}_{n}\left(\boldsymbol{\theta}_{\boldsymbol{n}}\right)$ é uma função de estimação não viesada se

$$
\mathrm{E}_{\theta}\left\{\boldsymbol{\Psi}_{n}(\boldsymbol{\theta})\right\}=0
$$

Se todas as funções de estimação $\boldsymbol{\psi}_{i}(\cdot), i=1,2, \ldots, n$ são não viesadas então, $\boldsymbol{\Psi}_{n}(\boldsymbol{\theta})=\sum_{i=1}^{n} \boldsymbol{\psi}_{i}(\boldsymbol{\theta})$ é não viesada. 
Definição 1.4 Seja $\boldsymbol{\Psi}_{n}\left(\boldsymbol{\theta}_{\boldsymbol{n}}\right)$ uma função de estimação não viesada. A matriz de variabilidade de $\boldsymbol{\Psi}_{n}\left(\boldsymbol{\theta}_{\boldsymbol{n}}\right)$ é definida por,

$$
\mathbf{V}_{\boldsymbol{\Psi}}(\boldsymbol{\theta})=E_{\theta}\left\{\boldsymbol{\Psi}_{n}(\boldsymbol{\theta}) \boldsymbol{\Psi}_{n}^{\top}(\boldsymbol{\theta})\right\}
$$

e a matriz de sensibilidade é definida por,

$$
\mathbf{S}_{\boldsymbol{\Psi}}(\boldsymbol{\theta})=E_{\theta}\left(\frac{\partial}{\partial \boldsymbol{\theta}^{\top}} \boldsymbol{\Psi}_{n}(\boldsymbol{\theta})\right)
$$

Definição 1.5 Uma função $\mathbf{\Psi}=\left(\boldsymbol{\Psi}_{1}, \ldots, \boldsymbol{\Psi}_{p}\right)^{\top}$ é dita regular se para todo $\boldsymbol{\theta}=\left(\theta_{1}, \ldots, \theta_{p}\right)^{\top} \in \Theta$ :

(i) a função $\Psi$ é não viesada.

(ii) A derivada parcial de $\boldsymbol{\Psi}_{n}(\boldsymbol{\theta})$ com relação a $\boldsymbol{\theta}$ existe quase certamente para todo $\mathbf{y} \in \chi$.

(iii) É possivel permutar o sinal de integração e diferenciação da seguinte forma:

$$
\frac{\partial}{\partial \theta_{i}} \int_{\chi} \boldsymbol{\Psi}(\theta) d P_{\theta}=\int_{\chi} \frac{\partial}{\partial \theta_{i}}[\mathbf{\Psi}(\theta)] d P_{\theta}
$$

(iv) $E_{\theta}\left\{\boldsymbol{\Psi}_{j}(\boldsymbol{\theta}) \boldsymbol{\Psi}_{k}(\boldsymbol{\theta})\right\} \in \mathbb{R}$ com $j, k=1, \ldots, p$ e $\mathbf{V}_{\Psi}(\boldsymbol{\theta})$ é positiva definida.

(v) $E_{\theta}\left\{\frac{\partial}{\partial \theta_{l}} \mathbf{\Psi}_{j}(\boldsymbol{\theta}) \frac{\partial}{\partial \theta_{m}} \mathbf{\Psi}_{k}(\boldsymbol{\theta})\right\} \in \mathbb{R}$, com $j, k, l, m=1, \ldots, p$ e $\mathbf{S}_{\Psi}(\boldsymbol{\theta})$ é não singular.

Definição 1.6 A matriz de informação de Godambe de $\boldsymbol{\theta}$ associada a $\boldsymbol{\Psi}_{n}$, uma função de estimação regular, é dada por

$$
\mathbf{J}_{\Psi}(\boldsymbol{\theta})=\mathbf{S}_{\Psi}(\boldsymbol{\theta})^{\top} \mathbf{V}_{\Psi}^{-1}(\boldsymbol{\theta}) \mathbf{S}_{\Psi}(\boldsymbol{\theta})
$$

A informação de Godambe desempenha o papel da informação de Fisher para as funções de estimação regulares.

Jørgensen e Labouriau (1994) estabeleceram condições para a normalidade assintótica de estimadores obtidos a partir de funções de estimação regulares. Artes (1997) desenvolveu um novo resultado nessa direção levando em consideração parâmetros multidimensionais, que será apresentado a seguir.

Teorema 1.7 (Artes, 1997) Considerando-se que,

a) $\mathbf{y}_{i}=\left(y_{i 1}, \ldots, y_{i s}\right)^{\top}, i=1,2, \ldots, n$, são vetores s-dimensionais, independentes e identicamente distribuídos;

b) existe uma função mensurável $f: \chi \times \Theta \rightarrow \Theta$, tal que $\boldsymbol{\psi}_{i}(\boldsymbol{\theta})=f\left(\mathbf{y}_{i}, \boldsymbol{\theta}\right), i=1, \ldots, n$;

c) $\boldsymbol{\psi}_{i}(\boldsymbol{\theta})$, é uma função de estimação regular;

d) $\boldsymbol{\psi}_{n}(\boldsymbol{\theta})=\sum_{i=1}^{n} \psi_{i}(\boldsymbol{\theta})$;

e) para $\delta,>0 E_{\boldsymbol{\theta}}=\left\{\sup _{\mathbf{h}:\|\mathbf{h}\| \leq \delta} \| \frac{\partial \boldsymbol{\psi}_{i}}{\partial \boldsymbol{\theta}}(\boldsymbol{\theta}+\mathbf{h})-\frac{\partial \boldsymbol{\psi}_{i}}{\partial \boldsymbol{\theta}}(\boldsymbol{\theta})\right\}=\phi_{\delta}$ e $\phi_{\delta} \rightarrow 0$ quando $\delta \rightarrow 0, i=$ $1, \ldots, n$; 
f) $\{\hat{\boldsymbol{\theta}}\}_{n=1}^{\infty}$ é uma sequência de raízes de $\boldsymbol{\Psi}_{n}(\theta), \theta \in \Theta$ e sob condições que garantam a existência de uma sequência de raizes de $\boldsymbol{\Psi}_{n}(\theta)$ que seja limitada em probabilidade, ou restrita a um conjunto compacto q.c. quando $n$ tende a infinito, vem que $\hat{\boldsymbol{\theta}}_{n} \stackrel{P}{\longrightarrow} \boldsymbol{\theta}$ quando $n \rightarrow \infty$. E ainda

$$
\sqrt{n}\left(\hat{\boldsymbol{\theta}}_{n}-\boldsymbol{\theta}\right) \stackrel{D}{\longrightarrow} N_{p}\left(\mathbf{0}, \mathbf{J}_{\psi}^{-1}(\boldsymbol{\theta})\right) .
$$

É do nosso interesse, o estudo realizado em Crowder (1987). O autor investiga uma classe particular de funções de estimação dadas por

$$
\boldsymbol{\Psi}_{n}(\boldsymbol{\theta})=\sum_{i=1}^{n} \mathbf{Q}_{i}(\boldsymbol{\theta}) \mathbf{u}_{i}\left(\mathbf{y}_{i}, \boldsymbol{\theta}\right)
$$

em que $\mathbf{Q}_{i}(\boldsymbol{\theta})$ é uma matriz não estocástica de pesos que, eventualmente, pode ser função de $\boldsymbol{\theta}$, enquanto $\mathbf{u}_{i}, i=1,2, \ldots, n$, são vetores com média zero, mutuamente independentes que satisfazem as condições de regularidade. Denotaremos esta classe por $\mathcal{L}$ e a exemplo do que já é feito na literatura (ver Venezuela (2008)) chamaremos de funções de estimação lineares. A classe de funções de estimação lineares geradas por $\mathbf{u}_{i}$ é definida por,

$$
\mathcal{L}(\mathbf{u})=\left\{\boldsymbol{\Psi}_{n}(\boldsymbol{\theta}) \in \mathcal{R}: \boldsymbol{\Psi}_{n}(\boldsymbol{\theta})=\sum_{i=1}^{n} \mathbf{Q}_{i}(\boldsymbol{\theta}) \mathbf{u}_{i}\left(y_{i}, \boldsymbol{\theta}\right)\right\}
$$

Crowder(1987) mostra ainda que a função de estimação ótima dentre as funções da classe $\mathcal{L}$ é obtida quando a matriz de pesos é dada por

$$
\mathbf{Q}_{i}^{*}(\boldsymbol{\theta})=E_{\theta}\left(\frac{\partial \mathbf{u}_{i}}{\partial \boldsymbol{\theta}}\right)^{\top} \operatorname{Cov}^{-1}\left(\mathbf{u}_{i}\right)
$$

Podemos escrever $\operatorname{Cov}\left(\mathbf{u}_{i}\right)=\operatorname{Var}\left(\mathbf{u}_{i}\right)^{1 / 2} \mathbf{R}\left(\mathbf{u}_{i}\right) \operatorname{Var}\left(\mathbf{u}_{i}\right)^{1 / 2}$, em que

$$
\operatorname{Var}\left(\mathbf{u}_{i}\right)=\operatorname{diag}\left\{\operatorname{Var}\left(u_{i 1}\right), \ldots, \operatorname{Var}\left(u_{i s}\right)\right\}
$$

e $\mathbf{R}\left(\mathbf{u}_{i}\right)$ a verdadeira matriz de correlação de $\mathbf{u}_{\mathbf{i}}$ com dimensão $s \times s$.

\subsection{Objetivos}

O objetivo principal deste trabalho é propor uma abordagem alternativa para analisar dados BS correlacionados com base em equações de estimação. Da classe ótima de funções de estimação proposta por Crowder (1987) (ver também, Godambe (1997) e Artes et al. (2000)), obtemos uma classe ótima para a análise de dados correlacionados em que as distribuições marginais são assumidas $\log$-BS e log-BS- $t$.

Para atender a esse objetivo, determinamos como objetivos auxiliares:

1. garantir boas propriedades para os estimadores e implementar um método iterativo que torne possível aplicar o trabalho desenvolvido a dados reais;

2. como as esperanças e as matrizes de variância-covariância nem sempre irão possuir formas 
fechadas, tornando-se necessário o uso de resoluções numéricas de integrais durante a programação, é do nosso interesse torná-las o mais simples possível;

3. propor resíduos padronizados e alguns procedimentos de diagnóstico, mais especificamente, distância de Cook, influência local sob três esquemas de perturbação diferentes: ponderação de casos, perturbação da variável resposta e perturbação de uma covariável.

\subsection{Contribuições}

As principais contribuições deste trabalho são: desenvolvimento de equações de estimação para dados de tempo de vida que se adequem a distribuição marginal pertença à família de distribuições $\log$-BS e log-BS-t.

Os dados correlacionados incluem diferentes estruturas de dados, tais como: observações multivariadas, dados agrupados, medidas repetidas, dados longitudinais, para mais detalhes ver Verbeke (2000). Os dados multivariados são aqueles que recebem mais atenção na literatura, por exemplo, Kundu et al. (2013) derivou a versão multivariada da distribuição BS e recentemente Marchant et al. (2016a) derivaram o modelo de regressão log-linear GBS multivariado, bem como, Marchant et al. (2016b) apresentaram procedimentos de diagnósticos para esses modelos. Outra abordagem foi apresentada por Villegas et al. (2011) que propôs modelos lineares mistos sob erros log-BS. No entanto, não foram publicados estudos que analisassem dados de BS correlacionados do ponto de vista de equações de estimação generalizadas. Essa abordagem pode ser preferivel quando o objetivo é comparar as respostas marginais esperadas, ver Alencar et al. (2012). Uma outra vantagem do uso de equações de estimação é o fato de que as estimativas dos parâmetros obtidos por EEGs são consistentes mesmo quando a estrutura de variância-covariância é especificada de forma incorreta.

É válido salientar a importância dos procedimentos de diagnóstico desenvolvidos (análise de resíduos, distância de Cook, influência local com três esquemas de perturbação diferentes: ponderação de casos, perturbação da variável resposta e perturbação de uma covariável) uma vez que permitem averiguar se as suposições iniciais do modelo ajustado são adequadas, evitando assim erros ao empregar a abordagem proposta.

\subsection{Organização do trabalho}

Esta tese contém cinco capítulos incluindo essa introdução. No Capítulo 2 propomos equações de estimação para modelos de regressão log-BS com medidas repetidas e/ou longitudinais. Consideramos a modelagem da média, assumindo homogeneidade do parâmetro de forma. Propomos algoritmo de estimação, estudos de simulação, bem como métodos de diagnóstico. Uma aplicação com dados referentes à produtividade de capital público dos 48 estados norte-americanos contíguos de 1970 a 1986, que não tinha sido analisada sob essa perspectiva, é apresentada. O Capítulo 3 contém o desenvolvimento de equações de estimação para os modelos log-BS- $t$. Aqui também procedimentos de diagnóstico são propostos. Para facilitar a compreensão iremos utilizar a notação de forma análoga aos Capítulos 2 e 3. Um conjunto de dados relativo a um estudo realizado na Escola de Educação Física e Esporte da Universidade de São Paulo (USP) ano de 2016, em que 70 corredores foram avaliados em corridas em esteiras em três períodos é analisado. O Capítulo 4 
traz extensões dos modelos estudados nos Capítulos 2 e 3, apresentando a modelagem conjunta da média e da dispersão. Finalmente, no Capítulo 5 conclusões e trabalhos futuros são apresentados. 


\section{Capítulo 2}

\section{Equações de Estimação para modelos de regressão $\log -\mathrm{BS}$}

Apresentamos nesse capítulo o desenvolvimento de equações de estimação para os parâmetros de um modelo log-BS com dados correlacionados de forma que os estimadores obtidos sejam consistentes e assintoticamente normais. As equações de estimação aqui sugeridas são baseadas na proposta discutida em Artes (1997) e Venezuela (2008). Estudamos ainda o processo iterativo, inferência e métodos de diagnóstico, tais como análise de resíduos, distância de Cook, influência local conformal sob três esquemas de perturbação diferentes, ponderação de casos, perturbação da variável resposta e perturbação individual das covariáveis. Aplicaremos a teoria desenvolvida a um conjunto de dados reais.

\subsection{Notação}

Considere uma amostra aleatória de $n$ unidades amostrais, $\mathbf{t}_{1}, \ldots, \mathbf{t}_{n}$, em que $\mathbf{t}_{i}=\left(\mathrm{t}_{i 1}, \ldots, \mathrm{t}_{i s}\right)^{\top}$ é o vetor de respostas para a $i$-ésima unidade experimental e admitimos que cada componente $\mathrm{t}_{i j} \sim \mathrm{BS}\left(\alpha, \beta_{i j}^{*}\right)$. Ou seja, vamos supor inicialmente a homogeneidade do parâmetro de forma. Norteados pelo o que é feito na literatura, utilizaremos a proposta de Rieck e Nedelman (1991) e vamos empregar o modelo log-linear para a distribuição BS, veja por exemplo Leiva et al. (2007) que estudam modelo de regressão log-BS com observações censuradas, Villegas et al. (2011) e Desmond et al. (2012) que estenderam o modelo de Rieck e Nedelman (1991) para incluir efeitos aleatórios.

Portanto, assumimos que cada componente $\mathrm{y}_{i j}=\log \left(\mathrm{t}_{i j}\right) \sim \log -\mathrm{BS}\left(\alpha, \mu_{i j}\right)$, isto é, sua distribuição marginal é dada por

$$
f\left(y_{i j}\right)=\frac{1}{\alpha \sqrt{2 \pi}} \cosh \left(\frac{y_{i j}-\mu_{i j}}{2}\right) \exp \left\{-2\left[\frac{1}{\alpha} \operatorname{senh}\left(\frac{y_{i j}-\mu_{i j}}{2}\right)\right]^{2}\right\}, y_{i j} \in \mathbb{R}
$$

$\alpha>0, i=1, \ldots, n, j=1, \ldots, s_{i}$. Sem perda de generalidade, assumiremos experimentos balanceados $s_{i}=s$.

Defina, $\mathbf{y}=\left(\mathbf{y}_{1}^{\top}, \ldots, \mathbf{y}_{n}^{\top}\right)^{\top}$. Seja $\mathbf{x}_{i j}=\left(x_{i j 1}, \ldots, x_{i j p}\right)^{\top}$ um vetor $p$ dimensional com valores de variáveis explicativas relacionadas aos parâmetros $\mu_{i j}$. Além disso, $\mathbf{X}_{i}=\left(\mathbf{x}_{i 1}, \ldots, \mathbf{x}_{i s}\right)^{\top}$. 


\subsection{Equações de estimação para a média}

\subsubsection{Derivação da função de estimação}

Consideremos que as médias $\mu_{i j}$ sejam modeladas tais que

$$
\mu_{i j}=\eta_{i j}=\mathbf{x}_{i j}^{\top} \boldsymbol{\beta}
$$

em que $\boldsymbol{\beta}=\left(\beta_{1}, \ldots, \beta_{p}\right)^{\top}$ é um vetor de parâmetros desconhecidos a serem estimados.

Iremos primeiramente construir uma função de estimação para modelos de regressão log-BS com estrutura longitudinal, utilizando a Definição 1.19. Com esse intuito é necessário determinar uma função $\mathbf{u}_{i}=\mathbf{u}_{i}\left(\mathbf{y}_{i}, \boldsymbol{\beta}\right)$, com média zero, mutuamente independentes e que satisfaça às propriedades das funções de estimação regulares tal que $\mathbf{u}_{i}^{\prime}$ s sejam variáveis aleatórias. Propomos que a função $u(\cdot)$ seja obtida a partir da função escore.

Definindo $\xi_{i j 1}=\frac{1}{\alpha} \cosh \left(\frac{y_{i j}-\mu_{i j}}{2}\right)$ e $\xi_{i j 2}=\frac{1}{\alpha} \operatorname{senh}\left(\frac{y_{i j}-\mu_{i j}}{2}\right)$, a função densidade de probabilidade pode ser escrita da seguinte forma:

$$
f\left(y_{i j} ; \mu_{i j}, \alpha\right)=\frac{1}{\sqrt{2 \pi}} \xi_{i j 1} \exp \left\{-2 \xi_{i j 2}^{2}\right\}
$$

e o logaritmo da função de verossimilhança em função de $\mu_{i j}$ fica dado por

$$
l\left(\mu_{i j} ; y_{i j}\right)=\frac{1}{2} \log (2 \pi)+\log \left(\xi_{i j 1}\right)-2 \xi_{i j 2}^{2} .
$$

Observe que,

$$
\begin{gathered}
\frac{\partial \xi_{i j 1}}{\partial \mu_{i j}}=\frac{1}{\alpha} \operatorname{senh}\left(\frac{y_{i j}-\mu_{i j}}{2}\right)\left(-\frac{1}{2}\right)=-\frac{1}{2} \xi_{i j 2} \\
\text { e } \frac{\partial \xi_{i j 2}}{\partial \mu_{i j}}=\frac{1}{\alpha} \cosh \left(\frac{y_{i j}-\mu_{i j}}{2}\right)\left(-\frac{1}{2}\right)=-\frac{1}{2} \xi_{i j 1} .
\end{gathered}
$$

Então,

$$
\begin{aligned}
\frac{\partial l\left(\mu_{i j} ; y_{i j}\right)}{\partial \mu_{i j}} & =\frac{1}{\xi_{i j 1}} \xi_{i j 2}\left(-\frac{1}{2}\right)-4 \xi_{i j 2} \xi_{i j 1}\left(-\frac{1}{2}\right) \\
& =-\frac{\xi_{i j 2}}{2 \xi_{i j 1}}+2 \xi_{i j 1} \xi_{i j 2} .
\end{aligned}
$$

Ou podemos reescrever,

$$
\begin{aligned}
\frac{\partial l\left(\mu_{i j} ; y_{i j}\right)}{\partial \mu_{i j}} & =-\frac{1}{2} \operatorname{tgh}\left(\frac{y_{i j}-\mu_{i j}}{2}\right)+\frac{2}{\alpha^{2}} \operatorname{senh}\left(\frac{y_{i j}-\mu_{i j}}{2}\right) \cosh \left(\frac{y_{i j}-\mu_{i j}}{2}\right) \\
& =-\frac{1}{2} \operatorname{tgh}\left(\frac{y_{i j}-\mu_{i j}}{2}\right)+\frac{1}{\alpha^{2}} \operatorname{senh}\left(y_{i j}-\mu_{i j}\right) .
\end{aligned}
$$


Portanto, a função escore do parâmetro $\mu_{i j}$ fica dada por,

$$
U_{\mu_{i j}}=-\frac{1}{2} \operatorname{tgh}\left(\frac{y_{i j}-\mu_{i j}}{2}\right)+\frac{1}{\alpha^{2}} \operatorname{senh}\left(y_{i j}-\mu_{i j}\right) \text {. }
$$

Então, tomamos $\mathbf{u}_{i}=\left(u_{i 1}, \ldots, u_{i s}\right)^{\top} \operatorname{com} u_{i j}=U_{\mu_{i j}}$ para a proposta de obter a função de estimação para o parâmetro de localização da distribuição log-BS. Desta forma, garantimos que a função escore está contida na classe $\mathcal{L}\left(\mathbf{u}_{i}\right)$. E $\mathbf{u}_{i}$ é tal que $\mathrm{E}\left(u_{i j}\right)=0$. Mais detalhes ver o Apêndice A.

Assumindo a dependência entre as observações de uma mesma unidade amostral, os termos da função de estimação ótima na classe $\mathcal{L}$ para $\boldsymbol{\beta}$, gerado por $\mathbf{u}_{\mathbf{i}}$, são dados por

$$
\mathrm{E}\left(\frac{\partial \mathbf{u}_{i}}{\partial \boldsymbol{\beta}^{\top}}\right)^{\top}=\mathbf{X}_{i}^{\top} \mathbf{N}_{i} \text { e } \operatorname{Cov}\left(\mathbf{u}_{i}\right)=\operatorname{Var}\left(\mathbf{u}_{i}\right)^{1 / 2} \operatorname{Corr}\left(\mathbf{u}_{i}\right) \operatorname{Var}\left(\mathbf{u}_{i}\right)^{1 / 2}=\boldsymbol{\Sigma}_{i}^{1 / 2} \operatorname{Corr}\left(\mathbf{u}_{i}\right) \boldsymbol{\Sigma}_{i}^{1 / 2},
$$

sendo $\mathbf{N}_{i}=\mathrm{E}\left(\frac{\partial \mathbf{u}_{i 1}}{\partial \boldsymbol{\mu}_{i}}\right)=\operatorname{diag}\left\{\mathrm{E}\left(\dot{u}_{i}\right), \ldots, \mathrm{E}\left(\dot{u}_{i s}\right)\right\}, \operatorname{com} \mathrm{E}\left(\dot{u}_{i j}\right)=\frac{1}{4} \mathrm{E}\left\{\operatorname{sech}^{2}\left(\frac{y_{i j}-\mu_{i j}}{2}\right)\right\}-\frac{1}{2}-\frac{1}{\alpha^{2}}$, $\operatorname{Corr}\left(\mathbf{u}_{i}\right)$ é a verdadeira matriz de correlação entre as medidas $u_{i 1}, \ldots, u_{i s}, \boldsymbol{\Sigma}_{i}=\operatorname{diag}\left\{\operatorname{Var}\left(u_{i 1}\right), \ldots\right.$, $\left.\operatorname{Var}\left(u_{i s}\right)\right\}, \operatorname{com} \operatorname{Var}\left(u_{i j}\right)=\mathrm{E}\left\{\frac{1}{4} \operatorname{tgh}^{2}\left(\frac{y_{i j}-\mu_{i j}}{2}\right)\right\}+\frac{1}{4}+\frac{1}{\alpha^{2}}$. Assim, a função de estimação linear ótima para $\boldsymbol{\beta}$ fica dada por,

$$
\mathbf{\Psi}_{n}^{*}(\boldsymbol{\beta})=\sum_{i=1}^{n} \mathbf{X}_{i}^{\top} \mathbf{N}_{\mathbf{i}} \operatorname{Cov}_{i}\left(\mathbf{u}_{i}\right)^{-1} \mathbf{u}_{\mathbf{i}}
$$

Os cálculos para obter a equação de estimação acima encontram-se no Apêndice B.1.

Na prática, a verdadeira matriz de correlação é desconhecida. Pela proposta de Liang e Zeger (1986), assumimos a existência do vetor $\rho(\ell \times 1)$ que caracteriza a matriz de correlação de trabalho, $\mathbf{R}(\rho)$. Logo, a função de estimação para $\boldsymbol{\beta}$ fica dada por,

$$
\mathbf{\Psi}_{n}(\boldsymbol{\beta})=\sum_{i=1}^{n} \mathbf{X}_{i}^{\top} \mathbf{N}_{i} \mathbf{C}_{i}^{-1} \mathbf{u}_{i}=\sum_{i=1}^{n} \mathbf{X}_{i}^{\top} \mathbf{W}_{\mathbf{i}} \mathbf{N}_{i}^{-1} \mathbf{u}_{i}
$$

em que $\mathbf{C}_{i}=\boldsymbol{\Sigma}_{i}^{1 / 2} \mathbf{R}(\rho) \boldsymbol{\Sigma}_{i}^{1 / 2}, \mathbf{W}_{i}=\mathbf{N}_{i} \mathbf{C}_{i}^{-1} \mathbf{N}_{i}$ e $\hat{\boldsymbol{\beta}}$ denota a raiz de (2.4).

\subsubsection{Algoritmo de estimação}

\section{Estimação de $\beta$}

Para encontrarmos o estimador $\hat{\boldsymbol{\beta}}$ de $\boldsymbol{\beta}$ (fixados $\alpha$ e $\rho$ ) devemos resolver a seguinte equação de estimação:

$$
\Psi_{n}(\hat{\boldsymbol{\beta}})=0
$$

ou, equivalentemente,

$$
\sum_{i=1}^{n} \mathbf{X}_{i}^{\top} \hat{\mathbf{W}}_{i} \hat{\mathbf{N}}_{i}^{-1} \hat{\mathbf{u}}_{i}=0
$$

Para determinar as raízes da equação (2.5), ou seja, o valor de $\hat{\boldsymbol{\beta}}$, utilizaremos o método de 
Newton-Raphson modificado (veja Jørgensen et al. (1996)), expandindo a EEG dada na equação (2.4) em torno de um valor inicial $\hat{\boldsymbol{\beta}}^{(0)}$, de forma que obtemos o seguinte processo iterativo:

$$
\begin{aligned}
\boldsymbol{\beta}^{(m+1)} & =\boldsymbol{\beta}^{(m)}-\left\{\mathrm{E}\left[\frac{\partial}{\partial \boldsymbol{\beta}^{\top}} \mathbf{\Psi}_{n}\left(\boldsymbol{\beta}^{(m)}\right)\right]\right\}^{-1} \mathbf{\Psi}_{n}\left(\boldsymbol{\beta}^{(m)}\right) \\
& =\boldsymbol{\beta}^{(m)}-\left\{\sum_{i=1}^{n} \mathbf{X}_{i}^{\top} \hat{\mathbf{N}}_{i} \hat{\mathbf{C}}_{i} \hat{\mathbf{N}}_{i} \mathbf{X}_{i}\right\}^{-(m)}\left\{\sum_{i=1}^{n} \mathbf{X}_{i}^{\top} \hat{\mathbf{N}}_{i} \hat{\mathbf{C}}_{i} \hat{\mathbf{u}}_{i}\right\}^{(m)} \\
& =\boldsymbol{\beta}^{(m)}-\left\{\sum_{i=1}^{n} \mathbf{X}_{i}^{\top} \hat{\mathbf{W}}_{i} \mathbf{X}_{i}\right\}^{-(m)}\left\{\sum_{i=1}^{n} \mathbf{X}_{i}^{\top} \hat{\mathbf{W}}_{i} \hat{\mathbf{N}}_{i}^{-1} \hat{\mathbf{u}}_{i}\right\}^{(m)}
\end{aligned}
$$

sendo $m=0,1,2, \ldots$ o número de iterações e $\hat{\mathbf{W}}_{i}=\hat{\mathbf{N}}_{i} \hat{\mathbf{C}}_{i} \hat{\mathbf{N}}_{i}$. As estimativas $\hat{\alpha}$ e $\hat{\rho}$ são fornecidas inicialmente e modificadas separadamente a cada passo do processo iterativo.

A equação (2.6) reescrita na forma do processo iterativo de mínimos quadrados reponderados fica expressa por

$$
\boldsymbol{\beta}^{(m+1)}=\left\{\left[\sum_{i=1}^{n} \mathbf{X}_{i}^{\top} \hat{\mathbf{W}}_{i} \mathbf{X}_{i}\right]^{-1}\left[\sum_{i=1}^{n} \mathbf{X}_{i}^{\top} \hat{\mathbf{W}}_{i} \hat{\mathbf{z}}_{i}\right]\right\}^{(m)}
$$

que emprega uma matriz de pesos $\mathbf{W}_{i}$ e uma variável dependente modificada $\mathbf{z}_{i}$, sendo $\mathbf{z}_{i}=\boldsymbol{\eta}_{i}-$ $\mathbf{N}_{i}^{-1} \mathbf{u}_{i}$.

\section{Estimação de $\alpha$}

Apresentamos a seguir dois estimadores que podem ser utilizados para estimar o parâmetros $\alpha$.

i) Estimador baseado na função de verossimilhança

Sob a hipótese de independência entre as observações de um mesmo indivíduo e dado $\boldsymbol{\beta}$, a função escore do parâmetro $\alpha$ fica dada por

$$
\mathrm{U}_{\alpha}=\sum_{i=1}^{n} \sum_{j=1}^{s} \frac{1}{\alpha}\left\{\frac{4}{\alpha^{2}} \operatorname{senh}^{2}\left(\frac{y_{i j}-\mu_{i j}}{2}\right)-1\right\},
$$

o estimador de $\alpha$ é a solução da equação $\mathrm{U}_{\alpha}=0$ e é dado por,

$$
\hat{\alpha}=\sqrt{\frac{\sum_{i=1}^{n} \sum_{j=1}^{s}\left\{4 \operatorname{senh}^{2}\left(\frac{y_{i j}-\hat{\mu}_{i j}}{2}\right)\right\}}{n s} .}
$$

ii) Método dos momentos modificados

Também assumindo a hipótese de independência entre as observações de um mesmo indivíduo, o método dos momentos modificados consiste em igualar $\mathrm{E}(T)$ e $\mathrm{E}\left(T^{-1}\right)$ aos seus respectivos momentos amostrais. Sejam $\mathbf{t}_{1}, \mathbf{t}_{2}, \ldots \mathbf{t}_{n}$ uma amostra aleatória de tamanho $n$ de uma distribuição BS. Defina, $r_{1}=\frac{1}{n s} \sum_{i=1}^{n} \sum_{j=1}^{s} t_{i j}$ e $r_{2}=\left\{\frac{1}{n s} \sum_{i=1}^{n} \sum_{j=1}^{s} t_{i j}^{-1}\right\}^{-1}$ o estimador 
obtido pelo método dos momentos modificados fica dado por,

$$
\hat{\alpha}=\left\{2\left[\left(\frac{r_{1}}{r_{2}}\right)^{1 / 2}-1\right]\right\}^{1 / 2}
$$

e possui distribuição limite dada por, $\hat{\alpha} \sim \mathrm{N}\left(\alpha, \frac{\alpha^{2}}{2 n s}\right)$. Maiores detalhes ver $\mathrm{Ng}$ et al. (2003).

\section{Estimação de $\rho$}

Considerando a estrutura de correlação desconhecida, $\rho$ será um vetor com $s(s-1) / 2$ componentes, em que $\rho_{j k}$, corresponde à correlação entre $u_{i j}$ e $u_{i k}$ e precisa ser estimado.

Liang e Zeger (1986) ressaltam que para qualquer estrutura da matriz de correlação de trabalho, $\mathbf{R}(\rho)$, utilizada, $\hat{\boldsymbol{\beta}}$ e $\hat{\mathbf{J}}_{n}^{-1}$ serão consistentes. A eficiência cresce quanto mais próxima da verdadeira matriz de correlação estiver a escolha da matriz de correlação de trabalho.

Artes (1997) propõe estimadores para algumas estruturas usadas para definir $\mathbf{R}(\rho)$.

Vamos supor então que $\mathbf{R}(\rho)=\left\{\mathrm{R}_{j, l}(\rho)\right\}$ para $j=1, \ldots, s$. Em particular se $\mathbf{R}(\rho)$ é permutável então, $\mathrm{R}_{j l}(\rho)=\rho, \forall j \neq l$ e $1 \leq j, l \leq t, \mathrm{R}_{j l}(\rho)=1$ para $j=l$. Então, a estimativa de $\rho$ fica dada por

$$
\hat{\rho}=\left\{\frac{\left(\sum_{i=1}^{n} \sum_{j>l}^{n} \hat{u}_{i j} \hat{u}_{i l}\right)}{\left(\sum_{i=1}^{n} \sum_{j>l}^{n} \hat{u}_{i j}^{2}\right)} \frac{n s}{\frac{1}{2} n s(s-1)}\right\}=\left\{\frac{\left(\sum_{i=1}^{n} \sum_{j>l}^{n} \hat{u}_{i j} \hat{u}_{i l}\right)}{\left(\sum_{i=1}^{n} \sum_{j>l}^{n} \hat{u}_{i j}^{2}\right)} \frac{2}{(s-1)}\right\} .
$$

Se a estrutura de correlação assumida for autoregressiva de primeira ordem, $\operatorname{AR}(1)$, então, $\mathbf{R}_{j l}(\rho)=$ $\rho^{|j-l|}, 1 \leq j, l \leq t$. Nesse caso, a estimativa de $\rho$ fica dada por

$$
\hat{\rho}=\left\{\frac{\left(\sum_{i=1}^{n} \sum_{j=1}^{s-1} \hat{u}_{i j} \hat{u}_{i j+1}\right)}{\left(\sum_{i=1}^{n} \sum_{j=1}^{s-1} \hat{u}_{i j}^{2} \sum_{i=1}^{n} \sum_{j=2}^{s} \hat{u}_{i j}^{2}\right)^{1 / 2}}\right\} .
$$

Quando a matriz de correlação é não estruturada, ou seja, $\mathrm{R}_{j l}(\rho)=\rho_{j l}$, utiliza-se o estimador dado por,

$$
\hat{\rho}_{j l}=\left\{\frac{\left(\sum_{i=1}^{n} \hat{u}_{i j} \hat{u}_{i l}\right)}{\left(\sum_{i=1}^{n} \hat{u}_{i j}^{2}\right)^{1 / 2}\left(\sum_{i=1}^{n} \hat{u}_{i l}^{2}\right)^{1 / 2}}\right\} .
$$

Os passos do processo iterativo para obter as estimativas de $\boldsymbol{\beta}, \alpha$ e $\rho$ ficam dados por:

\section{Processo iterativo}

1) Para determinar o chute inicial do processo iterativo, $\boldsymbol{\beta}^{(0)}$, supomos independência entre as observações da mesma unidade experimental e ajustamos um modelo log-BS;

2) para determinar valor inicial para $\alpha^{(0)}$, utilizamos $\boldsymbol{\beta}^{(0)}$ e estimamos $\alpha$ utilizando a equação (2.8) e estimamos $\rho$ a partir de alguma estrutura de correlação;

3) atualizamos $\boldsymbol{\beta}$ de (2.7);

4) atualizamos $\alpha$, de (2.8) e $\rho$ da estrutura de correlação selecionada;

5) repetimos os 3-4 até a convergência. 


\subsubsection{Inferência}

Como ressaltamos anteriormente, na prática, a verdadeira matriz de correlação não é conhecida. O Teorema 2.1, dado em Artes (1997) (Teorema 6, p. 47) apresenta condições suficientes para assegurar que $\hat{\boldsymbol{\beta}}$, obtido a partir de 2.4 , (ou seja, solução de $\boldsymbol{\Psi}_{n}(\hat{\boldsymbol{\beta}})=0$,) é um estimador consistente e assintoticamente normal de $\boldsymbol{\beta}$.

Teorema 2.1 Seja $\hat{\boldsymbol{\beta}}$ a raiz de 2.4, sob condições gerais de regularidade, e assumindo que

a) $\hat{\rho}(\boldsymbol{\beta}, \alpha)$ é um estimador $\sqrt{n}$-consistente de $\rho$, isto é, $\|\hat{\rho}(\boldsymbol{\beta}, \alpha)-\hat{\rho}\|=O_{p}\left(n^{-1 / 2}\right)$;

b) $\hat{\alpha}(\boldsymbol{\beta})$ é um estimador $\sqrt{n}$-consistente de $\alpha$;

c) $\|\partial \hat{\rho}(\boldsymbol{\beta}, \alpha) / \partial \alpha\| \leq \mathbf{H}(\mathbf{y}, \boldsymbol{\beta})$, sendo $\mathbf{H}(\mathbf{y}, \boldsymbol{\beta})$ uma função $O_{p}(1)$, então temos que $\hat{\boldsymbol{\beta}}$ é um estimador consistente de $\boldsymbol{\beta}$ e

$$
\sqrt{n}\left(\hat{\boldsymbol{\beta}}_{n}-\boldsymbol{\beta}\right) \stackrel{D}{\longrightarrow} N_{p}\left(\mathbf{0}, \mathbf{J}^{-1}\right)
$$

$\operatorname{com} \mathbf{J}=\lim _{n \longrightarrow \infty} \mathbf{J}_{n} / n$, sendo $\mathbf{J}_{n}$ a matriz de informação de Godambe de $\boldsymbol{\beta}$ associada à $\boldsymbol{\psi}(\cdot)$ e dada por

$$
\mathbf{J}_{n}=\left\{\sum_{i=1}^{n} \mathbf{S}_{i}\right\}\left\{\sum_{i=1}^{n} \mathbf{V}_{i}\right\}^{-1}\left\{\sum_{i=1}^{n} \mathbf{S}_{i}\right\} .
$$

Os cálculos detalhados para a obtenção de $\mathbf{S}_{i}(\boldsymbol{\beta})$ e $\mathbf{V}_{i}(\boldsymbol{\beta})$ são obtidas no Apêndice B.1.

Artes (1997) ressalta que a matriz de variância-covariância não precisa ser a verdadeira para que os resultados assintóticos do teorema sejam válidos, embora estar corretamente especificada pode gerar resultados mais eficientes.

A matriz de variância-covariância de $\hat{\boldsymbol{\beta}}$ pode ser consistentemente estimada por

$$
\hat{\mathbf{J}}_{n}^{-1}=\left(\sum_{i=1}^{n} \hat{\mathbf{S}}_{i}\right)^{-1}\left(\sum_{i=1}^{n} \mathbf{X}_{i}^{\top} \hat{\mathbf{N}}_{i} \hat{\mathbf{C}}_{i}^{-1} \hat{\mathbf{u}}_{i} \hat{\mathbf{u}}_{i}^{\top} \hat{\mathbf{C}}_{i}^{-1} \hat{\mathbf{N}}_{i} \mathbf{X}_{i}\right)\left(\sum_{i=1}^{n} \hat{\mathbf{S}}_{i}\right)^{-\top}
$$

conhecido como estimador robusto, empírico ou sanduíche.

Se também a matriz de correlação entre as observações $u_{i 1}, \ldots, u_{i s}$ estiver especificada corretamente, então o estimador se reduz a $\hat{\mathbf{J}}_{n}^{-1}=\left\{\sum_{i=1}^{n} \hat{\mathbf{S}}_{i}\right\}^{-1}$, chamado de estimador naive. O estimador robusto apresenta a vantagem de ser sempre consistente, enquanto que o estimador naive é consistente quando o 0ã o está corretamente especificado, a matriz de correlação de trabalho também deve estar correta. Em contrapartida, Firth (1992) destaca que o estimador naive é mais eficiente e Prentice (1988) conclui que para pequenas amostras o estimador naive possui melhores propriedades.

Segundo Johnston (1996), uma indicação que a matriz $\mathbf{R}(\rho)$ é adequada ocorre quando as estimativas naive e robusta são próximas.

Para avaliar o teste de hipóteses $\mathrm{H}_{0}: \mathbf{A} \boldsymbol{\beta}=\mathbf{0}$ contra $\mathrm{H}_{1}: \mathbf{A} \boldsymbol{\beta} \neq \mathbf{0}$, em que $\mathbf{A}$ é uma matriz $(r \times p)$ de rank $r(r \leq p)$, pode ser aplicado um teste tipo-Wald cuja respectiva estatística é dada 
por $\xi_{W}=\hat{\boldsymbol{\beta}}^{\top} \mathbf{A}^{\top} \widehat{\mathbf{J}}_{n} \mathbf{A} \hat{\boldsymbol{\beta}}$. Para grandes amostras e sob as usuais condições de regularidade segue que $\xi_{W} \sim \chi_{r}^{2}$, em que $\chi_{r}^{2}$ denota a distribuição qui-quadrado com $r$ graus de liberdade.

\subsubsection{Estudo de simulação}

Nesta seção, descrevemos um estudo de simulação para avaliar o desempenho dos estimadores dos parâmetros do modelo. Para gerar os dados, assumimos que $\mathbf{y}_{i}=\left(\mathrm{y}_{i 1}, \ldots, \mathrm{y}_{i s}\right)^{\top}$ segue uma distribuição multivariada log-BS, como descrito em Kundu et al. (2013), com vetor parâmetro de localização $\boldsymbol{\mu}_{i}=\left(\mu_{i 1}, \ldots, \mu_{i s}\right)^{\top}$, parâmetro de forma $\alpha$ e correlação $\rho$, para $i=1, \ldots, n$. Em adição, o componente sistemático é dado por

$$
\mu_{i j}=\beta_{0}+\beta_{1} x_{i j}
$$

em que $x_{i j}$ 's denotam valores fixos gerados de uma distribuição uniforme no intervalo $[0,1]$, para $i=1, \ldots, n$ e $j=1, \ldots, s$. Consideramos estruturas de correlação, autorregressiva de primeira ordem e permutável. Os valores para os parâmetros são $\beta_{0}=4, \beta_{1}=-2, \alpha=0,5$ e $\rho=0,3,0,6$ e 0,9 , tamanhos amostrais $n=10,20,50$ e 80 e $s=3,5$ e 10 . O viés relativo (VR) de $\beta_{j}$ é estimado como $100 \times\left|\bar{\beta}_{j}-\beta_{j}\right| / \beta_{j}$, com $\bar{\beta}_{j}=R^{-1} \sum_{r=1}^{R} \hat{\beta}_{j}^{(r)}$, com $\hat{\beta}_{j}^{(r)}$ sendo a estimativa EEG de $\beta_{j}$ na $r$-ésima réplica, para $j=0,1, \ldots, R$, e o erro quadrático médio (EQM) é dado por $R^{-1} \sum_{r=1}^{R}\left(\beta_{j}-\hat{\beta}_{j}^{(r)}\right)^{2}$. Consideramos para cada cenário $R=5000$ réplicas.

As tabelas com os resultados do estudo de simulação estão no Apêndice C. Na Tabela C.1 são apresentados os resultados do estudo de simulação quando geramos dados com estrutura de correlação autorregressiva de primeira ordem e utilizamos a mesma estrutura para ajustar os dados gerados e obter as estimativas, ou seja, escolhemos a estrutura de correlação correta. Na Tabela C.2 é obtida da mesma forma mas substituindo a estrutura de correlação autorregressiva de primeira ordem pela estrutura permutável. Finalmente, na Tabela C.3 encontram-se os resultados obtidos quando existe uma má especificação da estrutura de correlação, em que geramos dados com estrutura de correlação autorregressiva de primeira ordem e ajustamos considerando uma estrutura permutável.

Podemos observar nas Tabelas C.1 e C.2 que os vieses relativos de $\hat{\beta}_{0}$ e $\hat{\beta}_{1}$ são em geral menores do que $0,2 \%$ e apresentam valores muito aproximados de $\beta_{0}$ e $\beta_{1}$, respectivamente. É importante salientar que quando aumentamos o número de observações em cada indivíduo, $s$ (tamanho do cluster), o VR diminui. Quando analisamos as estimativas $\hat{\alpha}$ percebemos que vão para os valores verdadeiros mais lentamente. Mas, em geral, temos boas aproximações para $n=80$, em todos os cenários. As estimativas de $\rho$ também vão para os valores verdadeiros à medida que $n$ aumenta (Tabelas C.1 e C.2).

Na Tabela C.3 observamos comportamentos semelhantes no que diz respeito as estimativas dos parâmetros $\beta_{0}, \beta_{1}$ e $\alpha$. Entretanto, quando ocorre uma má especificação da estrutura de correlação temos um distanciamento do valor verdadeiro de $\rho$ estimado independente do tamanho da amostra.

Os erros quadráticos médios de $\hat{\beta}_{0}$ e $\hat{\beta}_{1}$ são, em geral, menores do que 0,1 e decrescem quando $n$ cresce (para $s$ e $\rho$ fixos) e quando $s$ cresce (para $n$ e $\rho$ fixos).O erro quadrático médio de $\hat{\alpha}$ bem como das estimativas $\hat{\beta}_{0}$ e $\hat{\beta}_{1}$ decresce quando $n$ cresce ou $s$ cresce, entretanto, cresce quando $\rho$ cresce. 


\subsubsection{Métodos de diagnóstico}

Para que a seleção do modelo seja feita de forma adequada são propostos diversos métodos com intuito de verificar a qualidade do ajuste. Os objetivos dos procedimentos de diagnóstico são avaliar a existência de afastamento das suposições feitas inicialmente para o modelo, bem como detectar observações discrepantes, especialmente, pontos influentes e outliers. Essas observações podem exercer grande influência sobre as estimativas dos parâmetros e em algumas situações podem causar alterações inferenciais.

Já existe na literatura uma série de métodos de diagnóstico para modelos desenvolvidos a partir da abordagem EEGs. Barnhart e Williamson (1998) propuseram testes robustos de bondade de ajuste para o método EEGs com respostas binárias. Horton et al. (1999) propuseram testes para respostas binárias repetidas usando decis de risco. Embora facilmente interpretável, Oh et al. (2008) chamam atenção para o fato do método proposto por Horton et al. (1999) não conseguir detectar importantes afastamentos do ajuste testando apenas covariáveis que estão no modelo. Lee e Qaqish (2004) apresentaram uma aproximação para respostas binárias repetidas em uma abordagem EEGs modificadas que substitui a matriz de covariância por uma matriz baseada em distribuição multinomial. Park e Lee (2004) propuseram gráficos de resíduos para investigar o ajuste do modelo para dados de medidas repetidas normalmente distribuídos. Os gráficos dos resíduos são baseados em gráficos quantil-quantil (Q-Q) de uma distribuição qui-quadrado e então Oh et al. (2008) estenderam os métodos propostos por Park e Lee (2004) para modelos baseados em EEGs com dados discretos. Esta metodologia é particularmente útil para comparar diversos modelos simultaneamente.

Venezuela et al. (2007), com o objetivo de analisar dados longitudinais discretos e contínuos, generalizaram medidas bem conhecidas: matriz de projeção, a distância de Cook e resíduos padronizados desenvolvidos para respostas independentes. Os autores propuseram ainda uma aproximação gráfica para identificação de outliers usando gráficos meio-normal e simulando envelopes. Venezuela et al. (2011) descrevem medidas de influência local para equações de estimação generalizadas.

Vens e Ziegler (2012) recomendam a utilização de EEGs num ensaio clínico longitudinal como uma alternativa para a análise padrão, salientando que as técnicas de diagnóstico para EEGs devem acompanhar a análise, os autores utilizam diagnósticos de deleção padrão e gráficos de resíduos aplicando uma abordagem para as parcelas meio-normais com envelopes simulados.

Neste trabalho, vamos discutir algumas extensões dos procedimentos desenvolvidos por Venezuela (2008) para os modelos estudados. As medidas de diagnóstico são construídas a partir dos processos iterativos reponderados usados para estimar parâmetros de regressão.

\section{Análise de resíduos}

Uma vez que a função de verossimilhança não é conhecida para os modelos propostos, os resíduos de componentes quantílicos e desvio não são possíveis de ser avaliados, a não ser para os modelos marginais, por isso vamos derivar um tipo de resíduo de Pearson. Na convergência do processo iterativo (2.7) obtemos

$$
\hat{\boldsymbol{\beta}}=\left(\mathbf{X}^{\top} \widehat{\mathbf{W}} \mathbf{X}\right)^{-1} \mathbf{X}^{\top} \widehat{\mathbf{W}} \widehat{\mathbf{z}}
$$


em que $\mathbf{X}=\left[\mathbf{X}_{1}^{\top}, \ldots, \mathbf{X}_{n}^{\top}\right]^{\top}$ é a matriz modelo $\left(\sum s \times p\right), \widehat{\mathbf{W}}=\operatorname{diag}\left\{\widehat{\mathbf{W}}_{1}, \ldots, \widehat{\mathbf{W}}_{n}\right\}$ é $\left(\sum s \times \sum s\right)$ matriz de pesos, enquanto $\mathbf{z}=\left(\mathbf{z}_{1}^{\top}, \ldots, \mathbf{z}_{n}^{\top}\right)^{\top} \operatorname{com} \mathbf{z}_{i}=\mathbf{X}_{i} \boldsymbol{\beta}-\mathbf{N}_{i}^{-1} \mathbf{u}_{i}$ sendo uma pseudo-resposta para o $i$-ésimo grupo, para $i=1, \ldots, n$. Portanto, $\hat{\boldsymbol{\beta}}$ pode ser interpretada como uma solução de mínimos quadrados da regressão linear de $\mathbf{W}^{1 / 2} \mathbf{z}$ tendo como matriz de planejamento $\mathbf{W}^{1 / 2} \mathbf{X}$.

Por conseguinte, a matriz de projeção ortogonal de $\widehat{\mathbf{z}}$ sobre o subespaço vetorial gerado pelas colunas de $\widehat{\mathbf{W}}^{1 / 2} \mathbf{X}$ passa a ser dada por

$$
\widehat{\mathbf{H}}=\operatorname{diag}\left\{\widehat{\mathbf{H}}_{1}, \ldots, \widehat{\mathbf{H}}_{n}\right\},
$$

$\operatorname{com} \mathbf{H}_{i}=\widehat{\mathbf{W}}_{i}^{\frac{1}{2}} \mathbf{X}_{i}\left(\mathbf{X}^{\top} \widehat{\mathbf{W}} \mathbf{X}\right)^{-1} \mathbf{X}_{i}^{\top} \widehat{\mathbf{W}}_{i}^{\frac{1}{2}}, \mathbf{H}_{i}$ sendo uma matriz $\left(\sum s \times \sum s\right)$, para $i=1, \ldots, n$. E assim, os elementos da diagonal principal da matriz $\mathbf{H}_{i}$, denotados por $\widehat{h}_{i j j}, j=1, \ldots, s$, podem ser considerados medidas individuais de influência. Também, podemos construir medidas de influência de grupos como $\bar{h}_{i}=\sum_{j=1}^{s} h_{i j j} / s$, para $i=1, \ldots, n$. Os gráficos de $\hat{h}_{i j j}$ e $\hat{h}_{i}$ contra os índices das observações podem ser úteis para revelar observações (ou grupos de observações) com alta influência nos próprios valores preditos.

Resíduos ordinários podem ser definidos como

$$
\begin{aligned}
\mathbf{r}_{i} & =\widehat{\mathbf{W}}_{i}^{1 / 2} \mathbf{z}_{i}-\widehat{\mathbf{W}}_{i}^{1 / 2} \mathbf{X}_{i} \widehat{\boldsymbol{\beta}}_{i} \\
& =\widehat{\mathbf{W}}_{i}^{\frac{1}{2}}\left(\widehat{\mathbf{z}}_{i}-\mathbf{X}_{i} \hat{\boldsymbol{\beta}}_{i}\right)
\end{aligned}
$$

Portanto,

$$
r_{i j}=\mathbf{e}_{i}^{\top} \widehat{\mathbf{W}}_{i}^{\frac{1}{2}}\left(\widehat{\mathbf{z}}_{i}-\mathbf{X}_{i} \hat{\boldsymbol{\beta}}_{i}\right),
$$

em que $\mathbf{e}_{i}$ é um vetor $\left(\sum s \times 1\right)$ de zeros com 1 na $j$-ésima posição. Também podemos escrever $r_{i j}=\widehat{\mathbf{W}}_{i}^{1 / 2} \hat{\mathbf{z}}_{i}-\mathbf{H}_{i} \widehat{\mathbf{W}}_{i}^{\frac{1}{2}} \hat{\mathbf{z}}_{i}$. Similarmente, definimos uma versão do resíduo padronizado

$$
t_{r_{i j}}=\frac{\mathbf{e}_{i}^{\top} \widehat{\mathbf{W}}_{i}^{\frac{1}{2}}\left(\widehat{\mathbf{z}}_{i}-\mathbf{X}_{i} \hat{\boldsymbol{\beta}}\right)}{\sqrt{1-\widehat{h}_{i j j}}}
$$

para $i=1, \ldots, n$ e $j=1, \ldots, s$. O gráfico normal de probabilidade para $t_{r_{i j}}$ pode revelar observações outliers, bem como a adequação do modelo proposto.

Para analisar o comportamento dos resíduos conduzimos um estudo de simulação baseado em 5000 amostras geradas a partir da distribuição multivariada log-BS com estrutura de correlação AR-1. Uma vez que os dados foram gerados ajustamos o modelo usando o método EEG e obtivemos os resíduos. Na Tabela 2.1, apresentamos algumas medidas para descrever os resíduos: média, desvio padrão, coeficiente de assimetria e curtose e a estatística de teste de bondade de ajuste de Kolmogorov-Sminorv para avaliar a normalidade nos resíduos. Podemos notar que os resíduos têm média próxima a zero, desvio padrão próximo a um, assimetria próxima a zero e curtose próximas de três. As Figuras 2.1 e 2.2 apresentam gráficos normais de probabilidade com respectivamente, $n=50$ e 100. A partir desses gráficos e das medidas descritivas temos que os resíduos têm distribuição aproximadamente normal. Comportamento similar foi obtido para outras estruturas de correlação. 
26

EQUAÇÕES DE ESTIMAÇÃO PARA MODELOS DE REGRESSÃO LOG-BS

2.2
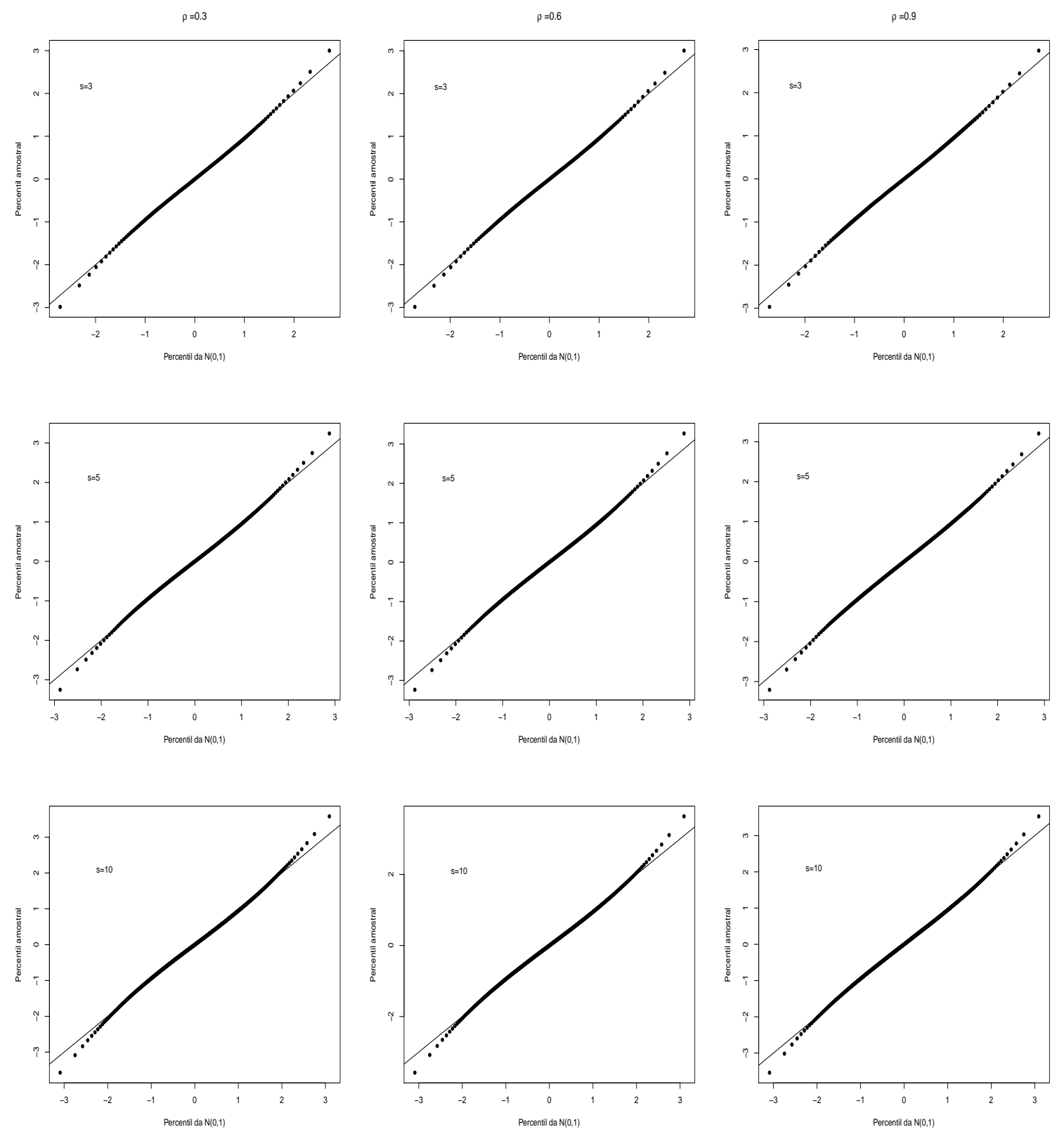

Figura 2.1: Gráficos normais de probabilidade para os resíduos padronizados para modelos log-BS ajustados via EEGs com amostras de tamanho $n=50$. 
2.2

EQUAÇÕES DE ESTIMAÇÃO PARA A MÉDIA

27
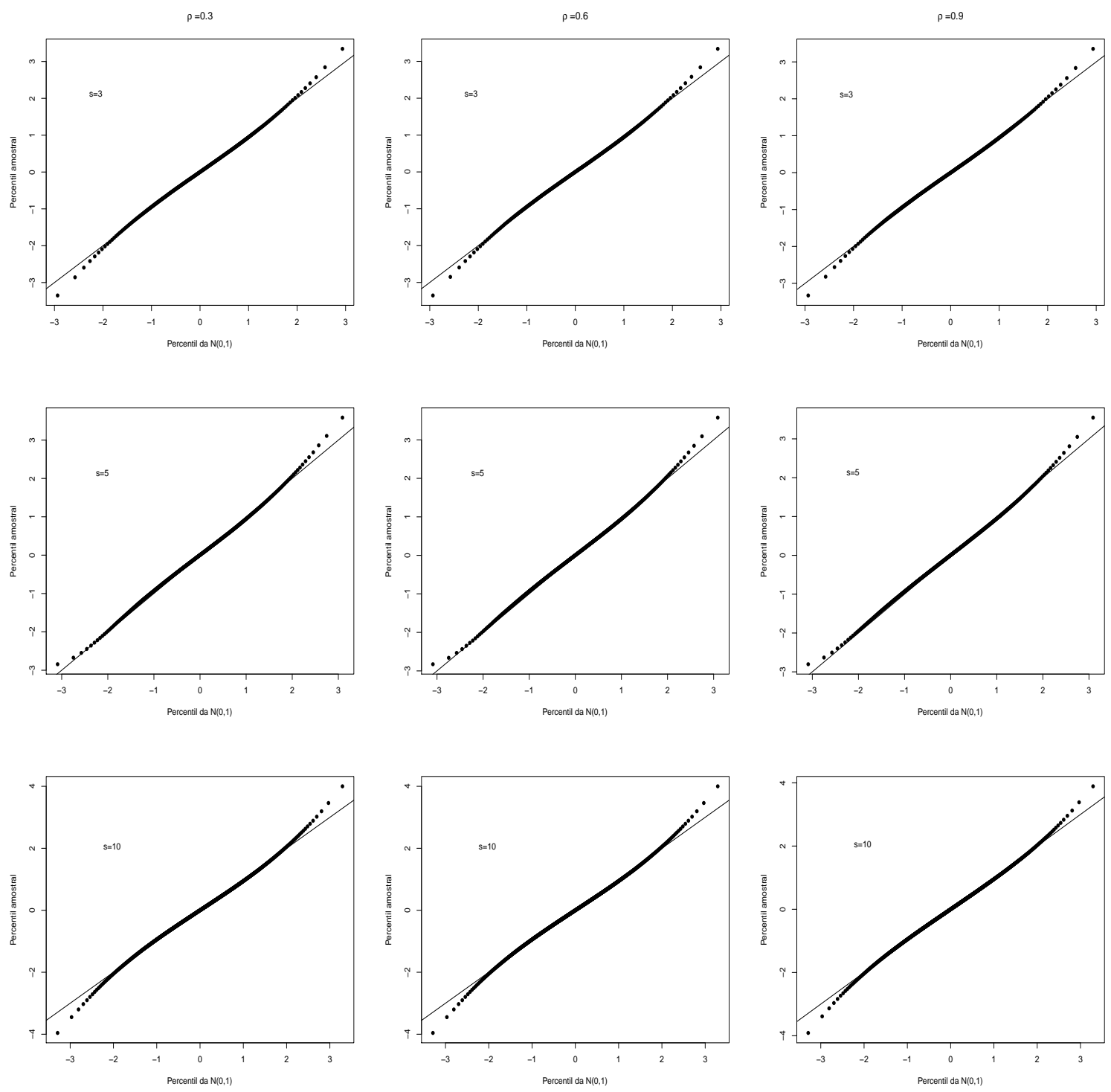

Figura 2.2: Gráficos normais de probabilidade para os resíduos padronizados para modelos log-BS ajustados via EEGs com amostras de tamanho $n=100$. 
Tabela 2.1: Medidas resumo da distribuição empírica dos resíduos tipo-Pearson padronizados do estudo de simulação no qual os dados foram gerados da distribuição BS com estrutura de correlação AR(1) e ajustado sob o modelo log-BS-EEG com a mesma estrutura de correlação.

\begin{tabular}{r|r|rccccc}
\hline & & \multicolumn{3}{|c}{$n=50$} & & \multicolumn{3}{c}{$n=100$} \\
\hline$\rho$ & & $s=3$ & $s=5$ & $s=10$ & $s=3$ & $s=5$ & $s=10$ \\
\hline \multirow{5}{*}{0,3} & Média & $-0,0001$ & $-0,0001$ & $<0,0001$ & $-0,0001$ & 0,0002 & $<0,0001$ \\
& Desvio Padrão & 1,0015 & 0,9876 & 0,9997 & 1,0005 & 0,9999 & 0,9997 \\
& Assimetria & 0,0102 & $-0,0011$ & 0,0002 & $-0,0079$ & 0,0039 & 0,0041 \\
& Curtose & 3,3707 & 3,4937 & 3,6100 & 3,5457 & 3,6320 & 3,7036 \\
& Estatística & 0,0183 & 0,0174 & 0,0173 & 0,0174 & 0,0180 & 0,0174 \\
\hline \multirow{5}{*}{0,6} & Média & $-0,0002$ & $-0,0002$ & 0,0002 & $-0,0003$ & 0,0001 & $-0,0001$ \\
& Desvio Padrão & 0,9997 & 0,9992 & 0,9983 & 0,9995 & 0,9987 & 0,9993 \\
& Assimetria & 0,0025 & 0,0013 & 0,0070 & $-0,0073$ & 0,0048 & 0,0008 \\
& Curtose & 3,3799 & 3,5060 & 3,6099 & 3,5499 & 3,6013 & 3,7242 \\
& Estatística & 0,0182 & 0,0182 & 0,0165 & 0,0178 & 0,0168 & 0,0165 \\
\hline \multirow{4}{*}{0,9} & Média & $-0,0004$ & 0,0006 & 0,0002 & 0,0001 & 0,0001 & $-0,0001$ \\
& Desvio Padrão & 0,9876 & 0,9886 & 0,9912 & 0,9927 & 0,9926 & 0,9960 \\
& Assimetria & $-0,0011$ & $-0,0051$ & $-0,0016$ & 0,0032 & 0,0039 & $-0,0053$ \\
& Curtose & 3,3688 & 3,4329 & 3,5094 & 3,5438 & 3,5644 & 3,5849 \\
& Estatística & 0,0199 & 0,01701 & 0,0152 & 0,0182 & 0,0162 & 0,0137 \\
\hline
\end{tabular}

\section{Distância de Cook}

Distância de Cook (Cook (1977)) é a medida de influência mais popular desenvolvida originalmente para modelos lineares para avaliar o efeito de excluir observações individuais sobre as estimativas de regressão. Pregibon (1981) estendeu o procedimento para modelos lineares generalizados pela introdução de uma aproximação de um passo para a obtenção de uma distância de Cook aproximada, enquanto Venezuela et al. (2007) aplica esse procedimento em equações de estimação generalizadas. Do processo iterativo (2.7), após algumas manipulações algébricas, obtemos a seguinte aproximação de um passo:

$$
\boldsymbol{\beta}_{(i j)}^{(1)}=\hat{\boldsymbol{\beta}}-\frac{r_{i j}}{\left(1-h_{i j j}\right)} \sqrt{\hat{\omega}_{i j}}\left(\mathbf{X}^{\top} \widehat{\mathbf{W}} \mathbf{X}\right)^{-1} \mathbf{x}_{i j}
$$

em que $\boldsymbol{\beta}_{(i j)}^{(1)}$ denota a aproximação para a estimativa $\hat{\boldsymbol{\beta}}_{(i j)}$, em que $\hat{\boldsymbol{\beta}}_{(i j)}$ é a estimativa do vetor paramétrico após remover a $j$-ésima medida da $i$-ésima unidade experimental. Então, a distância de Cook aproximada é dada por

$$
\begin{aligned}
\mathrm{C}_{i j} & =\frac{1}{p}\left(\boldsymbol{\beta}_{(i j)}^{(1)}-\hat{\boldsymbol{\beta}}\right)^{\top}\left(\mathbf{X}^{\top} \widehat{\mathbf{W}} \mathbf{X}\right)^{-1}\left(\boldsymbol{\beta}_{(i j)}^{(1)}-\hat{\boldsymbol{\beta}}\right) \\
& =\frac{1}{p} r_{i j}^{2} \frac{\hat{h}_{i j j}}{\left(1-\hat{h}_{i j j}\right)} .
\end{aligned}
$$

O gráfico de índices de $\mathrm{C}_{i j}$ pode revelar as observações influentes em $\hat{\boldsymbol{\beta}}$. 


\section{Influência conformal}

Cook (1986) introduziu o método de influência local como uma maneira geral de investigar a influência local dos casos. Influência local é um procedimento que tem como principal objetivo analisar o efeito de pequenas perturbações no modelo ou nos dados. Cadigan e Farrell (2002) generalizaram a abordagem de influência local de Cook (1986) e descreveram medidas de influência local que permitem avaliar o deslocamento de qualquer função de ajuste, como por exemplo a função de quase-verossimilhança. Venezuela et al. (2011), seguindo a proposta de Cadigan e Farrell (2002), desenvolveram medidas de influência local para equações de estimação generalizadas, definidas para modelos da regressão da média com medidas repetidas ou dados longitudinais.

Cadigan e Farrell (2002) descreveram a medida de influêncial local avaliando uma função de ajuste $\mathcal{F}(\boldsymbol{\theta})$ que é duas vezes diferenciável em $\boldsymbol{\theta}$ e que a estimativa de $\boldsymbol{\theta}$, denotada $\hat{\boldsymbol{\theta}}$, é solução de

$$
\boldsymbol{\Psi}(\hat{\boldsymbol{\theta}})=\left.\left[\frac{\mathcal{F}(\boldsymbol{\theta})}{\boldsymbol{\theta}}\right]\right|_{\boldsymbol{\theta}=\hat{\boldsymbol{\theta}}}=0
$$

De forma que a medida de afastamento da função de ajuste é a diferença entre a função de ajuste e a função de ajuste perturbada, $\left.\mathcal{F}\left(\hat{\boldsymbol{\beta}_{\omega}}\right)\right\}$, e fica dada por $\mathrm{FD}_{\omega}=2\left\{\mathcal{F}(\hat{\boldsymbol{\beta}})-\mathcal{F}\left(\hat{\boldsymbol{\beta}_{\omega}}\right)\right\}$, com $\boldsymbol{\omega}=$ $\left(\omega_{1}, \ldots, \omega_{q}\right)^{\top}$ sendo o vetor de perturbação e $\hat{\boldsymbol{\beta}}_{\omega}$ é a estimativa que maximiza a função de ajuste perturbada.

Então, Venezuela (2008), considerando a função $\mathbf{\Psi}(\boldsymbol{\beta})$, o vetor gradiente de uma função ajuste, propôs uma medida de influência local para equação de estimação definida por $d_{\max }$ correspondendo ao maior autovalor da matriz

$$
\mathbf{B}=-\boldsymbol{\Delta}^{\top} \mathbf{S}^{-1} \boldsymbol{\Delta}
$$

em que

$$
\boldsymbol{\Delta}=\frac{\partial \boldsymbol{\Psi}(\boldsymbol{\theta} \mid \boldsymbol{\omega})}{\partial \boldsymbol{\omega}^{\top}} \text { e } \mathbf{S}=\mathrm{E}(\ddot{\mathcal{F}})=\mathrm{E}\left(\frac{\partial \boldsymbol{\Psi}(\boldsymbol{\theta})}{\partial \boldsymbol{\theta}^{\top}}\right)
$$

$\operatorname{com} \boldsymbol{\theta}=\hat{\boldsymbol{\theta}}$ e $\boldsymbol{\omega}=\boldsymbol{\omega}_{0}$. Poon e Poon (1999) propuseram estudar a curvatura normal conformal na direção $\mathbf{d}$, no ponto $\boldsymbol{\omega}_{0}$ pertencente ao gráfico de $\alpha$ por

$$
B_{\mathbf{d}}=-\frac{\mathbf{d}^{\top} \boldsymbol{\Delta}^{\top} \ddot{\mathcal{F}}^{-1} \boldsymbol{\Delta} \mathbf{d}}{\sqrt{\operatorname{tr}\left(\boldsymbol{\Delta}^{\top} \ddot{\mathcal{F}}^{-1} \boldsymbol{\Delta}\right)^{2}}} .
$$

A exemplo de Cadigan e Farrell (2002) e Venezuela (2008) vamos considerar o valor esperado $\mathrm{E}(\ddot{\mathcal{F}})=\mathbf{S}$, uma vez que nem sempre é fácil determinar $\ddot{\mathcal{F}}$. Baseado em Venezuela (2008) e Poon e Poon (1999), a medida de influência local conformal será determinada por

$$
B_{\mathbf{d}}=-\frac{\mathbf{d}^{\top} \boldsymbol{\Delta}^{\top} S^{-1} \boldsymbol{\Delta} \mathbf{d}}{\sqrt{\operatorname{tr}\left(\boldsymbol{\Delta}^{\top} S^{-1} \boldsymbol{\Delta}\right)^{2}}} .
$$

Aqui, apresentamos a matriz $\Delta$ para três esquemas de perturbação, a saber: ponderação de casos, perturbação na variável resposta e perturbação na variável explicativa. 
i) Ponderação de casos

A função de estimação estudada é dada por,

$$
\Psi(\boldsymbol{\beta})=\mathbf{X}^{\top} \mathbf{W} \mathbf{N}^{-1} \mathbf{u}
$$

então, para o esquema de perturbação de ponderação de casos, a equação de estimação fica dada por

$$
\boldsymbol{\Psi}(\boldsymbol{\beta} \mid \boldsymbol{\omega})=\mathbf{X}^{\top} \mathbf{W} \mathbf{N}^{-1} \operatorname{diag}(\boldsymbol{\omega}) \mathbf{u}
$$

em que $\boldsymbol{\omega}=\left(\boldsymbol{\omega}_{1}^{\top}, \ldots, \boldsymbol{\omega}_{n}^{\top}\right)^{\top} \operatorname{com} \boldsymbol{\omega}_{i}=\left(\omega_{i 1}, \ldots, \omega_{i s}\right)^{\top}, i=1, \ldots, n$ e o vetor que representa a ausência de perturbação, $\boldsymbol{\omega}_{0}$ que nesse caso, assume 1 para todo $i=1, \ldots, n$ e $j=1, \ldots, s$.

Então,

$$
\boldsymbol{\Delta}=\frac{\partial \boldsymbol{\Psi}(\boldsymbol{\beta} \mid \boldsymbol{\omega})}{\partial \boldsymbol{\omega}^{\top}}=\mathbf{X}^{\top} \mathbf{W} \mathbf{N}^{-1} \operatorname{diag}(\mathbf{u})
$$

avaliados em $\hat{\boldsymbol{\theta}}=\left(\hat{\alpha}, \hat{\boldsymbol{\beta}}^{\top}\right)^{\top}$ e $\boldsymbol{\omega}=\boldsymbol{\omega}_{0}$.

ii) Perturbação da variável resposta

Consideramos aqui que cada $y_{i j}$ é perturbado da seguinte forma:

$$
y_{\omega i j}=y_{i j}+\omega_{i j} s_{y_{i j}}
$$

em que $\omega_{i j}=0, \forall i=1, \ldots, n, j=1, \ldots, s$ denota a ausência da perturbação e $s_{y_{i j}}$ pode ser estimado por $\sqrt{\operatorname{Var}\left(y_{i j}\right)}$. A função de estimação perturbada assume a forma,

$$
\Psi(\boldsymbol{\beta} \mid \boldsymbol{\omega})=\mathbf{X}^{\top} \mathbf{W} \mathbf{N}^{-1} \mathbf{u}_{\omega}
$$

em que $\mathbf{u}_{\omega}=\left(\mathbf{u}_{\omega 1}, \ldots, \mathbf{u}_{\omega n}\right)^{\top}$ e o vetor perturbado da variável resposta para a $i$-ésima unidade experimental é $\mathbf{u}_{\omega i}=\left(u_{\omega i 1}, \ldots, u_{\omega i s}\right)^{\top}$ com

$$
u_{\omega i j}=-\frac{1}{2} \operatorname{tgh}\left(\frac{y_{\omega i j}-\mu_{i j}}{2}\right)+\frac{1}{\alpha^{2}} \operatorname{senh}\left(y_{\omega i j}-\mu_{i j}\right) .
$$

Observe que o índice $\omega$ indica que o vetor $\mathbf{u}$ depende da perturbação definida em (2.15). Salientamos que daqui em diante, sempre que um vetor ou matriz possuir índice $\omega$ queremos dizer que o mesmo depende do esquema de perturbação que estiver sendo discutido.

Então, nesse caso,

$$
\boldsymbol{\Delta}=\frac{\partial \boldsymbol{\Psi}(\boldsymbol{\beta} \mid \boldsymbol{\omega})}{\partial \boldsymbol{\omega}^{\top}}=\mathbf{X}^{\top} \mathbf{W} \mathbf{N}^{-1} \frac{\partial \mathbf{u}_{\omega}}{\partial \boldsymbol{\omega}^{\top}}
$$

avaliados em $\hat{\boldsymbol{\theta}}=\left(\hat{\alpha}, \hat{\boldsymbol{\beta}}^{\top}\right)^{\top}$ e $\boldsymbol{\omega}_{0}$. Sendo a forma matricial, $\frac{\partial \mathbf{u}}{\partial \boldsymbol{\omega}^{\top}}=\dot{\mathcal{U}} \operatorname{com} \dot{\mathcal{U}}=\operatorname{diag}\left\{\dot{\mathcal{U}}_{1}, \ldots, \dot{\mathcal{U}}_{n}\right\}$, em que $\dot{\mathcal{U}}_{i}=\operatorname{diag}\left\{\frac{\partial u_{\omega i 1}}{\partial \omega_{i 1}}, \ldots, \frac{\partial u_{\omega i s}}{\partial \omega_{i s}}\right\}$ com

$$
\frac{\partial u_{\omega i j}}{\partial \omega_{i j}}=\left[\frac{1}{\alpha^{2}} \cosh \left(y_{\omega i j}-\mu_{i j}\right)-\frac{1}{4} \operatorname{sech}^{2}\left(\frac{y_{\omega i j}-\mu_{i j}}{2}\right)\right] s_{y_{i j}} .
$$


iii) Perturbação em uma covariável

Consideramos o esquema de perturbação aditiva para $k$-ésima coluna da matriz $\mathbf{X}$ (assumida contínua). Então, cada componente de $\mathbf{x}_{\omega k}$ é dada por

$$
x_{\omega i j k}=x_{i j k}+\omega_{i j} s_{x k},
$$

em que $\sigma_{x k}$ é o desvio padrão de $\mathbf{x}_{k}$. Portanto,

$$
\mu_{\omega i j}=\beta_{1} x_{i j 1}+\beta_{2} x_{i j 2}+\ldots+\beta_{k}\left(x_{i j k}+\omega_{i j k} s_{x k}\right)+\ldots+\beta_{p} x_{p i j} .
$$

Observe que podemos escrever

$$
\Psi(\boldsymbol{\beta})=\mathbf{X}^{\top} \mathbf{N} \mathbf{C}^{-1} \mathbf{u}
$$

$\operatorname{com} \mathbf{C}=\boldsymbol{\Sigma}^{\frac{1}{2}} \mathbf{R}(\boldsymbol{\rho}) \boldsymbol{\Sigma}^{\frac{1}{2}}$.

Então, a equação de estimação perturbada fica dada por

$$
\boldsymbol{\Psi}(\boldsymbol{\beta} \mid \boldsymbol{\omega})=\mathbf{X}_{\omega}^{\top} \mathbf{N}_{\omega} \mathbf{C}_{\omega}^{-1} \mathbf{u}_{\omega}
$$

$\operatorname{com} \mathbf{C}=\boldsymbol{\Sigma}_{\omega}^{\frac{1}{2}} \mathbf{R}(\boldsymbol{\rho}) \boldsymbol{\Sigma}_{\omega}^{\frac{1}{2}}$. Portanto,

$$
\frac{\partial \mathbf{\Psi}(\boldsymbol{\mu} \mid \boldsymbol{\omega})}{\partial \omega_{i j}}=\mathbf{X}_{\omega}^{\top} \mathbf{N}_{\omega}\left[\frac{\partial \mathbf{C}_{\omega}^{-1}}{\partial \omega_{i j}} \mathbf{u}_{\omega}+\mathbf{C}_{\omega}^{-1} \frac{\partial \mathbf{u}_{\omega}}{\partial \omega_{i j}}\right]+\left[\frac{\partial \mathbf{X}_{\omega}^{\top}}{\partial \omega_{i j}} \mathbf{N}_{\omega}+\mathbf{X}_{\omega}^{\top} \frac{\partial \mathbf{N}_{\omega}^{-1}}{\partial \omega_{i j}}\right] \mathbf{C}_{\omega}^{-1} \mathbf{u}_{\omega}
$$

em que

$$
\frac{\partial \mathbf{C}_{\omega}^{-1}}{\partial \omega_{i j}}=-\mathbf{C}_{\omega}^{-1} \frac{\partial \mathbf{C}_{\omega}}{\partial \omega_{i j}} \mathbf{C}_{\omega}^{-1}
$$

$\operatorname{com} \mathbf{C}_{\omega}=\boldsymbol{\Sigma}_{\omega}^{1 / 2} \mathbf{R} \boldsymbol{\Sigma}_{\omega}^{1 / 2}$.

Então, precisamos das derivadas de $\mathbf{N}_{\omega}, \mathbf{C}_{\omega}$ e $\mathbf{u}_{\omega}$ em relação a $\boldsymbol{\omega}^{\top}$. Temos que,

$$
\frac{\partial \mathbf{N}_{\omega}}{\partial \omega_{i j}}=\frac{\partial \mathbf{C}_{\omega}}{\partial \omega_{i j}}=0
$$

No Apêndice B detalhamos os cálculos para obter os resultados aqui apresentados. E temos

$$
\frac{\partial u_{\omega i j}}{\partial \omega_{i j}}=\left\{\frac{1}{4} \operatorname{sech}^{2}\left(\frac{y_{i j}-\mu_{\omega i j}}{2}\right)-\frac{1}{\alpha^{2}}\left[1+2 \operatorname{senh}^{2}\left(\frac{y_{i j}-\mu_{\omega i j}}{2}\right)\right]\right\} s_{x k} .
$$

Portanto,

$$
\frac{\partial \mathbf{\Psi}(\boldsymbol{\mu} \mid \boldsymbol{\omega})}{\partial \omega_{i j}}=\mathbf{X}_{\omega}^{\top} \mathbf{N}_{\omega} \mathbf{C}_{\omega}^{-1} \frac{\partial \mathbf{u}_{\omega}}{\partial \omega_{i j}}+\frac{\partial \mathbf{X}_{\omega}^{\top}}{\partial \omega_{i j}} \mathbf{N}_{\omega} \mathbf{C}_{\omega}^{-1} \mathbf{u}_{\omega}
$$

avaliados em $\hat{\boldsymbol{\theta}}=\left(\hat{\alpha}, \hat{\boldsymbol{\beta}}^{\top}\right)^{\top}$ e $\boldsymbol{\omega}_{0}$. 


\subsubsection{Aplicação}

Consideremos o conjunto de dados descrito em Munnell (1990) sobre a produtividade de capital público dos 48 estados norte-americanos contíguos de 1970 a 1986. Esse conjunto de dados foi analisado por Greene (2012) e Manghi et al. (2016), respectivamente, considerando os modelos: normal linear e modelos lineares mistos elípticos. Vamos analisar novamente esse conjunto de dados através do ponto de vista de equações de estimação generalizadas. Em particular, iremos investigar a relação entre produto interno bruto (PIB) e o capital das empresas de saneamento básico (CESB) através dos anos. Sejam $\mathbf{t}_{i}=\left(\mathrm{t}_{i 1}, \ldots, \mathrm{t}_{i 17}\right)^{\top}$ e $\mathbf{x}_{i}=\left(x_{i 1}, \ldots, x_{i 17}\right)^{\top}$ os valores do PIB e do CESB, respectivamente, para o $i$-ésimo estado continental dos Estados Unidos da América durante 17 anos, com $i=1, \ldots, 48$. Na Figura 2.3 (esquerda) está a densidade do PIB, ignorando os valores da variável explicativa CESB. Observamos que há uma indicação de distribuição assimétrica à direita, enquanto a Figura 2.3 (direita) sugere uma tendência linear entre o logaritmo natural de CESB e o logaritmo natural do PIB. Na Figura 2.4 observa-se que o PIB aumenta com o aumento do capital das empresas de saneamento básico. Não tem-se evidência que a iteração do ano com o aumento do capital das empresas de saneamento básico tenha efeito sobre o PIB. Devido à estrutura de séries temporais dos dados, vamos assumir uma estrutura de correlação autoregressiva de primeira ordem entre as respostas de cada estado.
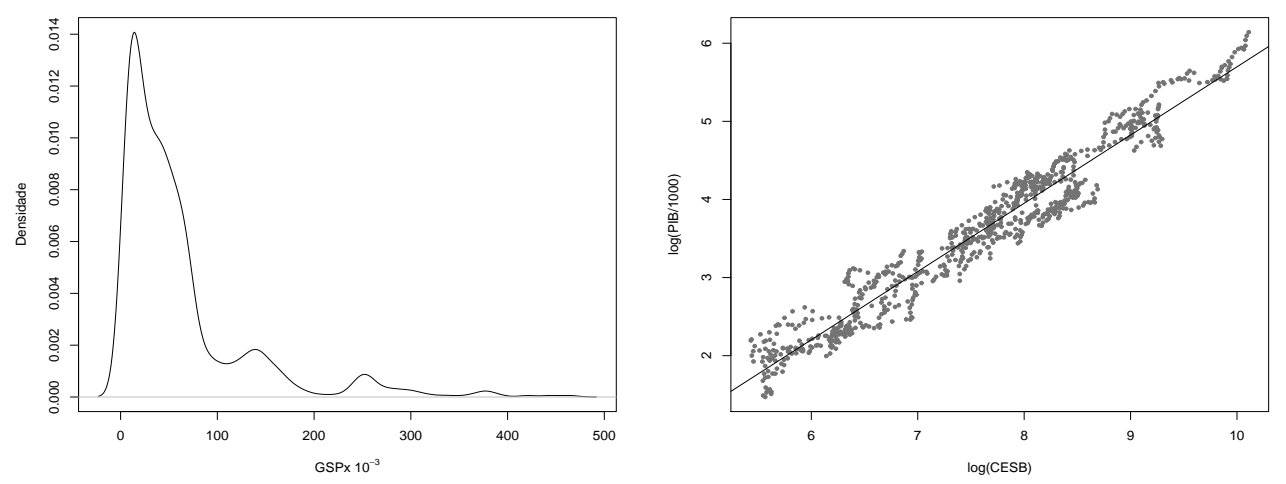

Figura 2.3: Densidade do PIB (esquerda) e dispersão entre o logaritmo natural do CESB e o logaritmo natural do PIB (direita).

Propomos, para analisar os dados, o seguinte modelo:

(i) $y_{i j} \sim \log -\mathrm{BS}\left(\alpha, \mu_{i j}\right)$,

(ii) $\mu_{i j}=\beta_{0}+\beta_{1} \log \left(x_{i j}\right)$,

(iii) $\mathbf{R}_{i}=\mathbf{R}_{i}(\rho)$ com $\mathbf{R}_{i j j^{\prime}}=1$, para $j=j^{\prime}$, e $\mathbf{R}_{i j j^{\prime}}=\rho^{\left|j-j^{\prime}\right|}$, se $j \neq j^{\prime}$,

em que $\mathrm{y}_{i j}=\log \left(\mathrm{t}_{i j}\right), \mu_{i j}$ e $\alpha>0$ denotam, respectivamente, a média e o parâmetro de forma, para $i=1, \ldots, 48$ e $j=1, \ldots, 17$. Esse é um modelo log-BS com estrutura de correlação AR-1, que iremos denominar modelo log-BS-EEG. A Tabela 2.2 apresenta as estimativas dos parâmetros, obtidos utilizando equações de estimação generalizadas, cujos os coeficientes são altamente significativos.

Os gráficos índices de $\left|B_{\text {dmax }}\right|$ para os esquemas de perturbação: ponderação de casos, perturbação da variável resposta, perturbação individual da covariável são apresentados na Figura 2.5. 

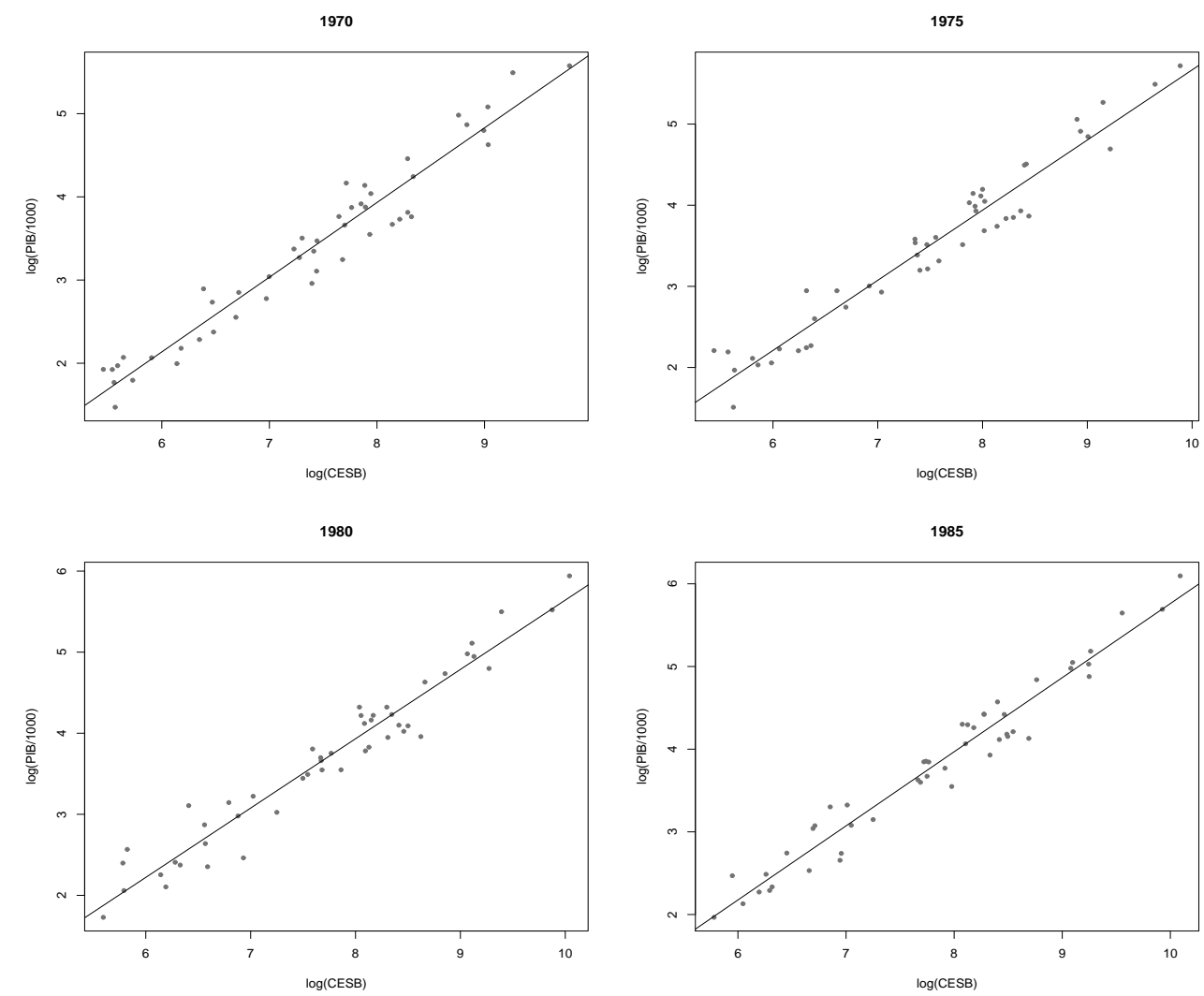

Figura 2.4: Dispersão entre o logaritmo natural do CESB e o logaritmo natural do PIB nos anos 1970, 1975,1980 e 1985.

Como podemos verificar as observações \#(7,3), \#(17,8), \#(17,9), \#(17,10), \#(39, 16) e \#(39,17) são classificadas como potencialmente influentes. A Tabela 2.3 mostra as mudanças relativas (MR) das estimativas após excluir os indivíduos com observações que são consideradas potencialmente influentes. Os valores $p$ para as novas estimativas estão entre parênteses. A MR (em porcentagem) para cada parâmetro estimado é definido por: $M R_{j}=\left[\hat{\beta}_{j}-\hat{\beta}_{j(I)}\right] / \hat{\beta}_{j} \times 100 \%$, com $\hat{\beta}_{j(I)}$ denotando o estimador de $\beta_{j}$ após o conjunto I de observações ser removido. Observe que $M R<2,3 \%$ para todas as estimativas do modelo e não ocorre mudança inferencial.

Da Figura 2.6 (esquerda) podemos perceber que os resíduos padronizados, em geral, estão no intervalo [-2,2]. O gráfico normal de probabilidades com o envelope gerado, apresentado na Figura 2.6 (direita) para os resíduos padronizados não apresentam indícios de afastamentos da distribuição assumida. Portanto, é razoável assumir que marginalmente $\mathrm{t}_{i j} \sim \operatorname{BS}\left(\alpha, \eta_{i j}\right)$, com $\eta_{i j}=x_{i j} \beta_{1} e^{\beta_{0}}$ denotando a mediana do PIB no $i$-ésimo estado continental do Estados Unidos no $j$-ésimo ano. Portanto, das estimativas dadas na Tabela 2.2, podemos concluir que para mudança de uma unidade no $\log (\mathrm{CESB})$ a mediana do PIB muda aproximadamente $e^{0,744}=2,105(105 \%)$.

Realizamos também uma nova análise dos dados em estudos utilizando modelos mistos desenvolvidos na Tese de Villegas (2010). Consideramos então o modelo log-BS misto dado por:

$$
\begin{aligned}
\mathrm{y}_{i j} \mid b_{i} & \stackrel{i n d}{\sim} \log -\operatorname{BS}\left(\alpha, \mu_{i j}\right), \\
b_{i} & \stackrel{i i d}{\sim} N(0, \zeta),
\end{aligned}
$$



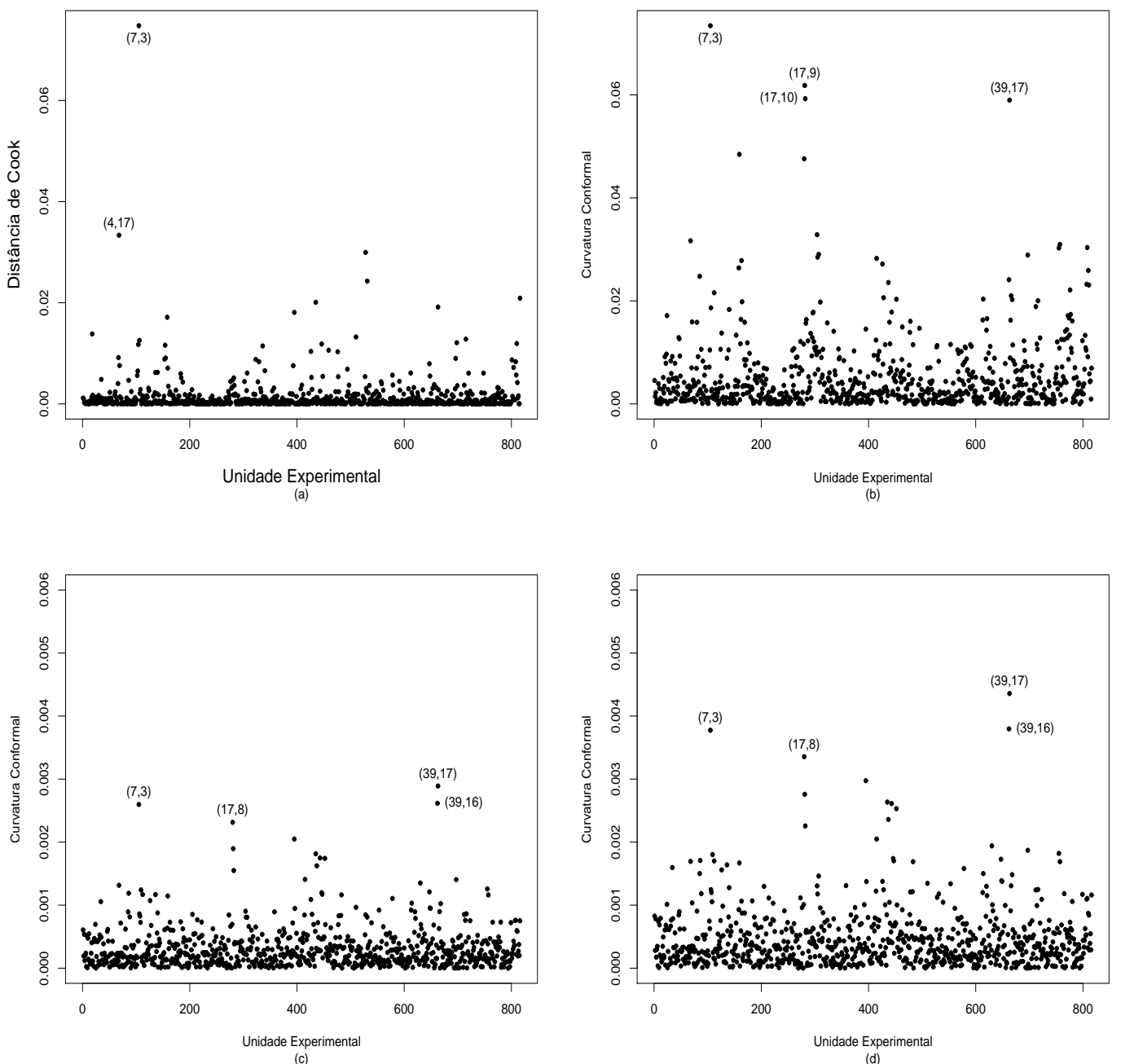

Figura 2.5: Gráfico da distância de Cook (a) e gráficos de influência local conformal segundo os seguintes esquemas de perturbação: ponderação de casos (b), perturbação da variável resposta (c) e perturbação individual das covariáveis(d).

Tabela 2.2: Estimativas dos parâmetros e erros padrão aproximados para o modelo log-BS-EEG ajustado aos dados sobre produtividade de capital público.

\begin{tabular}{lrrrr}
\hline & \multicolumn{4}{c}{ Modelo log-BS-EEG } \\
\hline Parâmetro & Estimativa & Erro padrão & z-robusto & valor p \\
\hline$\beta_{0}$ & 4,848 & 0,264 & 18,36 & $<0,01$ \\
$\beta_{1}$ & 0,744 & 0,035 & 21,26 & $<0,01$ \\
$\alpha$ & 0,236 & & & \\
$\rho$ & 0,979 & & & \\
\hline
\end{tabular}



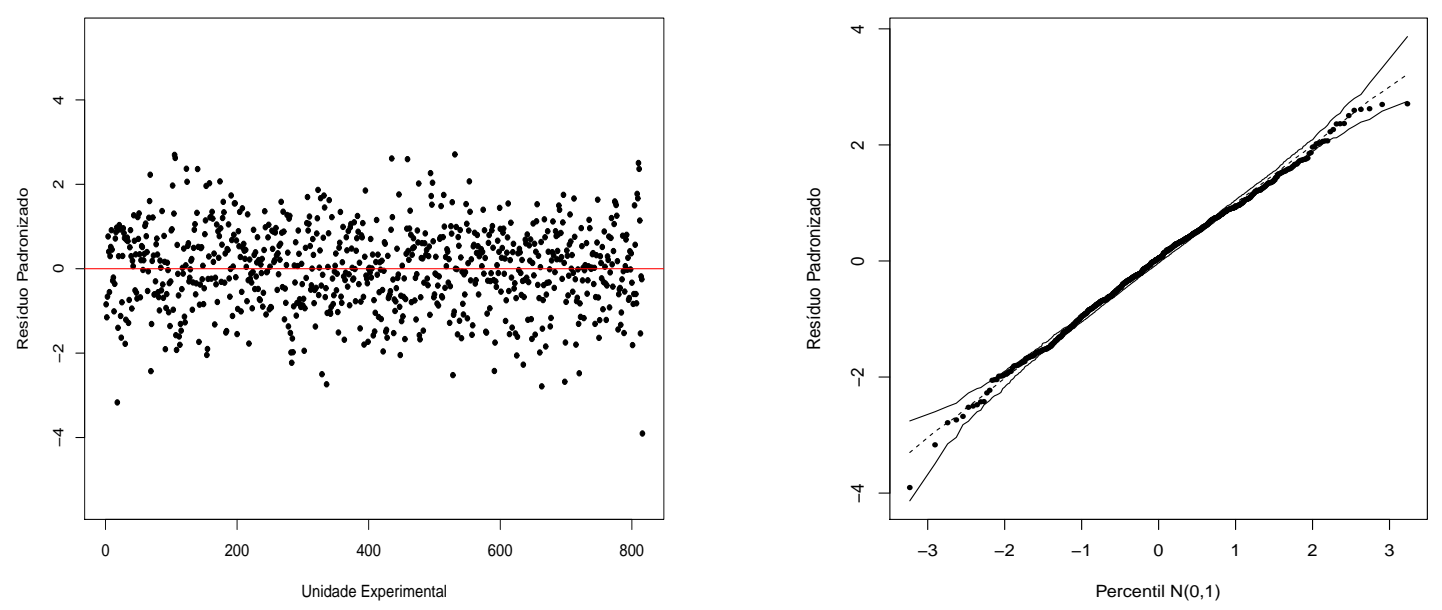

Figura 2.6: Gráfico de resíduos de Pearson padronizados (esquerdo) e gráfico normal de probabilidades para o resíduo de Pearson padronizado (direito) referente ao modelo log-BS-EEG gerado aos dados sobre produtividade do capital público.

Tabela 2.3: $M R$ (em \%) e correspondentes valores $p$ entre parênteses.

\begin{tabular}{lrrrr}
\hline Coeficientes & \multicolumn{4}{c}{ Indivíduos excluídos } \\
\cline { 2 - 5 } estimados & 7 & 4 & 17 & 39 \\
\hline$\hat{\beta}_{0}$ & $-0,8561$ & 2,2530 & $-1,2413$ & $-0,2648$ \\
& $(<0,01)$ & $(<0,01)$ & $(<0,01)$ & $(<0,01)$ \\
$\hat{\beta}_{1}$ & 0,7946 & $-2,1383$ & 1,2079 & 0,2907 \\
& $(<0,01)$ & $(<0,01)$ & $(<0,01)$ & $(<0,01)$ \\
$\hat{\alpha}$ & 0,3423 & 0,2621 & $-1,6162$ & $-0,4033$ \\
$\hat{\rho}$ & 0,0857 & $-0,0003$ & $-0,0271$ & $-0,0283$ \\
\hline
\end{tabular}


Tabela 2.4: Estimativas dos parâmetros e erro padrão aproximado para o modelo log-BS misto ajustado a partir do banco de dados em estudo.

\begin{tabular}{lrrrl}
\hline \multicolumn{5}{c}{ Modelo log-BS misto } \\
\hline Parâmetro & Estimativa & Erro padrão & z-robusto & \\
\hline$\beta_{0}$ & 4,4075 & 0,0337 & 130,6466 & $<0,01$ \\
$\beta_{1}$ & 0,8040 & 0,0045 & 179,8883 & $<0,01$ \\
$\alpha$ & 0,0899 & & & \\
\hline
\end{tabular}
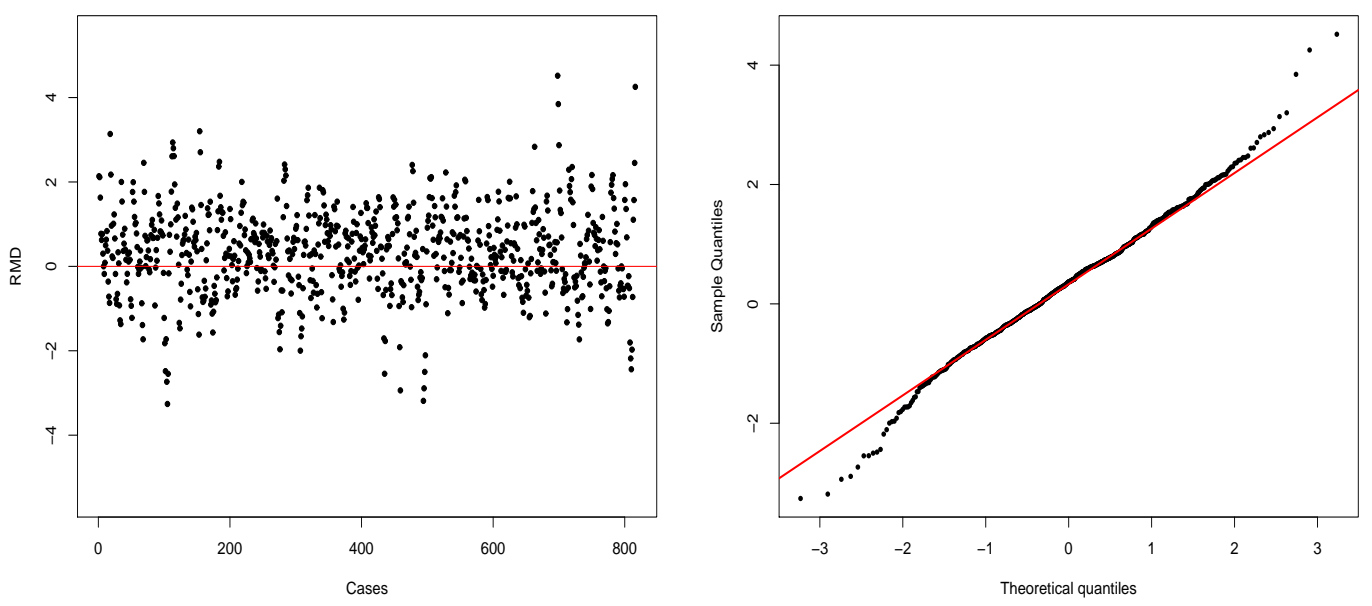

Figura 2.7: Gráficos dos resíduos tipo Martingale (esquerda) e gráfico normal de probabilidades (direita) para o modelo log-BS de intercepto aleatório gerado aos dados sobre produtividade do capital público.

em que $y_{i j}$ é o logaritmo do PIB do $i$-ésimo estado americano no $j$-ésimo tempo, $\mu_{i j}=\mu+b_{i}+\beta_{0}+$ $\beta_{1}$ em que $b_{i}$ representa o efeito aleatório associado ao $i$-ésimo estado americano. As estimativas de máxima verossimilhança dos parâmetros do modelo foram calculadas baseadas no logaritmo da função de verossimilhança, aproximada pela quadratura de Gauss Hermite com 50 pontos de quadratura, e foi empregado o algoritmo de quase-Newton, detalhes ver Villegas (2010) Os resultados das estimativas são apresentados na Tabela 2.4, e temos ainda $\hat{\zeta}=0,0456$ e $\hat{\rho}=0,9577$. Na Figura 2.7 apresentamos uma análise de resíduos tipo martigale.

Comparando os gráficos de resíduos nas Figuras 2.6 e 2.7, respectivamente, obtidos utilizando a abordagem EEG e a abordagem de modelos mistos e a Figura 2.8 concluimos que que a análise de dados utilizando metodologia EGG é mais adequada para os dados em estudo devido ao comportamento dos resíduos. 

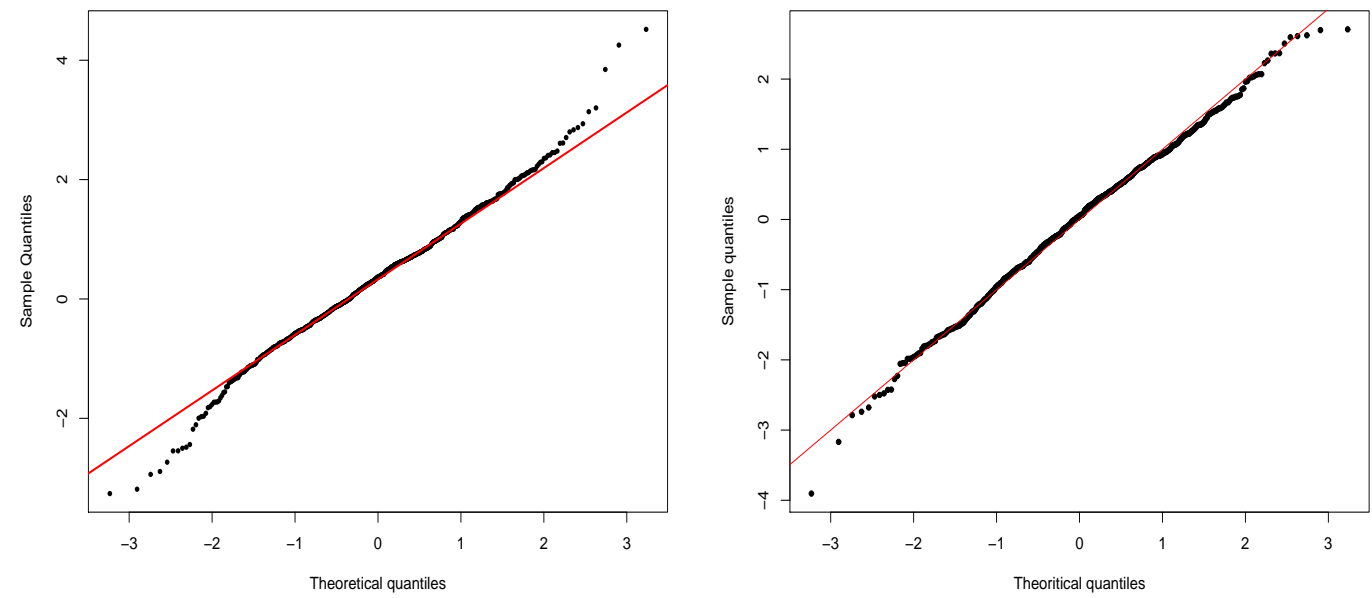

Figura 2.8: Gráfico normal de probabilidades para o modelo log-BS de intercepto aleatório (esquerda) e Gráfico normal de probabilidades para o modelo log-BS-EEG (direita) gerados aos dados sobre produtividade do público. 


\section{Capítulo 3}

\section{Equações de Estimação para modelos de regressão $\log -\mathrm{BS}-t$}

Com o intuito de produzir estimativas menos sensíveis a observações aberrantes, Barros et al. (2008) apresentaram modelos BS- $t$, que surgem como caso particular da distribuição BSG. Baseados em Barros et al. (2008) propomos, neste capítulo, a abordagem de equações de estimação generalizadas para os parâmetros de um modelo log-BS- $t$ com dados correlacionados de forma que os estimadores obtidos sejam consistentes e assintoticamente normais. Essas equações são obtidas considerando inicialmente a modelagem da média, supondo homogeneidade do parâmetro de forma. Seguimos a metodologia do Capítulo 2, desenvolvemos o processo iterativo e métodos de diagnóstico como resíduos, distância de Cook e influência local. Por fim, analisamos um conjunto de dados relativo a um estudo realizado na Escola de Educação Física e Esporte da Universidade de São Paulo (USP) no primeiro semestre de 2016, em que 70 corredores foram avaliados em corridas em esteiras em três períodos. A análise foi feita sob a perspectiva de EEGs considerando os modelos log-BS e $\log$-BS- $t$.

\subsection{Notação}

Assim, como no Capítulo 2, consideramos uma amostra aleatória de $n$ unidades amostrais, $\mathbf{t}_{1}, \ldots, \mathbf{t}_{n}$, em que $\mathbf{t}_{i}=\left(\mathbf{t}_{i 1}, \ldots, \mathbf{t}_{i s_{i}}\right)^{\top}$, com $\mathrm{t}_{i j} \sim \mathrm{BS}-t\left(\alpha, \beta_{i j}^{*}, \nu\right)$. Então, dada uma amostra aleatória de $n$ unidades amostrais, $\mathbf{y}_{1}, \ldots, \mathbf{y}_{n}, \operatorname{com} \mathbf{y}_{i}=\left(\mathrm{y}_{i 1}, \ldots, \mathrm{y}_{i s_{i}}\right)^{\top}$, assumimos que cada componente $\mathrm{y}_{i j}=\log \left(\mathrm{t}_{i j}\right)$ segue a distribuição $\log$-BS- $t\left(\alpha, \mu_{i j}, \nu\right)$ cuja função densidade de probabilidade de $\mathrm{y}_{i j}$ fica dada por

$$
f\left(y_{i j}, \mu_{i j}, \alpha\right)=\mathrm{c}(\nu)\left\{\frac{1}{\alpha} \cosh \left(\frac{y_{i j}-\mu_{i j}}{2}\right)\right\}\left\{1+\frac{1}{\nu}\left[\frac{2}{\alpha} \operatorname{senh}\left(\frac{y_{i j}-\mu_{i j}}{2}\right)\right]^{2}\right\}^{-\left(\frac{\nu+1}{2}\right)}
$$

em que

$$
\mathrm{c}(\nu)=\frac{\Gamma\left(\frac{\nu+1}{2}\right)}{(\nu \pi)^{1 / 2} \Gamma\left(\frac{\nu}{2}\right)}
$$


$\alpha>0, i=1, \ldots, n, j=1, \ldots, s_{i}$. Sem perda de generalidade, assumimos experimentos balanceados $s_{i}=s$.

Defina, $\mathbf{y}=\left(\mathbf{y}_{1}^{\top}, \ldots, \mathbf{y}_{n}^{\top}\right)^{\top}$ e $\mathbf{x}_{i j}=\left(x_{i j 1}, \ldots, x_{i j p}\right)^{\top}$ um vetor $p$-dimensional com valores de variáveis explicativas relacionadas com os parâmetros $\mu_{i j}$. Além disso, $\mathbf{X}_{i}=\left(\mathbf{x}_{i 1}^{\top}, \ldots, \mathbf{x}_{i s}^{\top}\right)^{\top}$.

\subsection{Equações de estimação para a média}

\subsubsection{Derivação da função de estimação}

Assim como foi feito no Capítulo 2, nosso primeiro objetivo é construir uma função de estimação para modelos de regressão log-BS- $t$ com estrutura longitudinal, utilizando a definição (1.19). Com esse propósito determinaremos uma função $\mathbf{u}_{i}=\mathbf{u}_{i}\left(\mathbf{y}_{i}, \boldsymbol{\beta}\right)$, com média zero, mutuamente independentes e que satisfaça às propriedades das funções de estimação regulares, tal que $\mathbf{u}_{i}$ 's sejam regulares.

Vamos supor inicialmente que $\mathrm{y}_{i j} \sim \log$-BS-t $\left(\alpha, \mu_{i j}, \nu\right)$, assumimos que $\nu$ é um parâmetro conhecido e consideramos que os parâmetros $\mu_{i j}$ sejam modeladas por

$$
\mu_{i j}=\eta_{i j}=\mathbf{x}_{i j}^{\top} \boldsymbol{\beta}
$$

em que $\boldsymbol{\beta}=\left(\beta_{1}, \ldots, \beta_{p}\right)^{\top}$ é um vetor de parâmetros a serem estimados.

Definindo $\xi_{i j 1}=\frac{1}{\alpha} \cosh \left(\frac{y_{i j}-\mu_{i j}}{2}\right)$ e $\xi_{i j 2}=\frac{1}{\alpha} \operatorname{senh}\left(\frac{y_{i j}-\mu_{i j}}{2}\right)$, a função densidade de probabilidade de $y_{i j}$ fica dada por

$$
f\left(y_{i j}, \mu_{i j}, \alpha\right)=\mathrm{c}(\nu) \xi_{i j 1}\left\{1+\frac{4}{\nu} \xi_{i j 2}^{2}\right\}^{-\left(\frac{\nu+1}{2}\right)}
$$

O logaritmo da função de verossimilhança em função de $\mu_{i j}$ fica dado por,

$$
l\left(\mu_{i j}, \alpha ; y_{i j}\right)=\log (\mathrm{c}(\nu))+\log \left[\xi_{i j 1}\right]-\left(\frac{\nu+1}{2}\right) \log \left[1+\frac{4}{\nu} \xi_{i j 2}^{2}\right] .
$$

Note que

$$
\frac{\partial \xi_{i j 1}}{\partial \mu_{i j}}=\frac{1}{\alpha} \operatorname{senh}\left(\frac{y_{i j}-\mu_{i j}}{2}\right)\left(-\frac{1}{2}\right)=-\frac{1}{2} \xi_{i j 2}
$$

$\mathrm{e}$

$$
\frac{\partial \xi_{i j 2}}{\partial \mu_{i j}}=\frac{1}{\alpha} \cosh \left(\frac{y_{i j}-\mu_{i j}}{2}\right)\left(-\frac{1}{2}\right)=-\frac{1}{2} \xi_{i j 1}
$$

De modo que,

$$
\begin{aligned}
\frac{\partial l\left(\mu_{i j}, \alpha ; y_{i j}\right)}{\partial \mu_{i j}} & =\frac{1}{\xi_{i j 1}} \xi_{i j 2}\left(-\frac{1}{2}\right)-\frac{\left(\frac{\nu+1}{2}\right)\left\{-\frac{1}{\nu} 4 \xi_{i j 2} \xi_{i j 1}\right\}}{\left\{1+\frac{4}{\nu} \xi_{i j 2}^{2}\right\}} \\
& =-\xi_{i j 2}\left(\frac{1}{2 \xi_{i j 1}}-\frac{2(\nu+1) \xi_{i j 1}}{\nu+4 \xi_{i j 2}^{2}}\right)
\end{aligned}
$$




$$
=-\xi_{i j 2}\left(\frac{1}{2 \xi_{i j 1}}-2 w_{i j} \xi_{i j 1}\right)
$$

em que $w_{i j}=\frac{\nu+1}{\nu+4 \xi_{i j 2}^{2}}$.

Portanto, a função escore para o parâmetro $\mu_{i j}$ fica dada por,

$$
u_{i j}=-\xi_{i j 2}\left(\frac{1}{2 \xi_{i j 1}}-2 w_{i j} \xi_{i j 1}\right)
$$

Como podemos notar a quantidade $w_{i j}$ que aparece na função escore (3.1) atua como um peso, assim como no caso independente, e isso faz com que o procedimento de estimação seja robusto, uma vez que observações com valores grandes para $\xi_{i j 2}^{2}$ devem receber pesos menores no processo de estimação. Mais detalhes ver Paula et al. (2012). Temos que $\mathrm{E}\left(u_{i j}\right)=0$. Então, podemos definir $\mathbf{u}_{i}=\left(u_{i 1}, \ldots, u_{i s}\right)^{\top}$, tal que $\mathrm{E}\left(u_{i j}\right)=0$ e $\operatorname{Cov}\left(\mathbf{u}_{i}\right)=\mathbf{W}_{i}$. Desta forma, a função escore está contida na classe $\mathcal{L}\left(\mathbf{u}_{i}\right)$.

Com o propósito de estruturarmos nossa função de estimação precisamos determinar $\left(\frac{\partial \mathbf{u}_{i}}{\partial \boldsymbol{\theta}}\right)^{\top} \mathrm{e}$ $\operatorname{Cov}^{-1}\left(\mathbf{u}_{i}\right)$. A fim de facilitar a compreensão deste texto e a execução computacional, vamos manter a notação utilizada no Capítulo 2. Denotamos então,

$$
\mathbf{N}_{i}=\mathrm{E}\left(\frac{\partial \mathbf{u}_{i}}{\partial \boldsymbol{\mu}_{i}}\right)=\operatorname{diag}\left\{\mathrm{E}\left(\dot{u}_{i 1}\right), \ldots, \mathrm{E}\left(\dot{u}_{i s}\right)\right\}
$$

em que

$$
\dot{u}_{i j}=\frac{1}{4+4 \xi_{i j 2}^{2} \alpha^{2}}-w_{i j}\left(\frac{1}{\alpha^{2}}+2 \xi_{i j 2}^{2}\right)+8 \xi_{i j 2}^{2} w_{i j}\left(\frac{1}{\alpha^{2}}+\xi_{i j 2}^{2}\right) \frac{1}{\left(\nu+4 \xi_{i j 2}^{2}\right)} .
$$

Vale salientar o fato que a forma que optamos por escrever $\dot{u}_{i j}$ na expressão (3.2) permite que mudanças de variáveis sejam realizadas e $\mathrm{E}\left(\dot{u}_{i j}\right)$ se torne mais eficiente para implementar computacionalmente uma vez que não é em função de funções hiperbólicas. Portanto,

$$
\begin{aligned}
\mathrm{E}\left(\dot{u}_{i j}\right)=\int_{-\infty}^{\infty} & \left\{\frac { 2 } { \alpha } \mathrm { c } ( \nu ) \{ 1 + \frac { 4 } { \nu } ( \frac { x _ { i j } } { \alpha } ) ^ { 2 } \} ^ { - ( \frac { \nu + 1 } { 2 } ) } \left[\frac{1}{4+4 x_{i j}^{2}}-w_{i j}\left(\frac{1+2 x_{i j}^{2}}{\alpha^{2}}\right)\right.\right. \\
& \left.\left.+8 \frac{x_{i j}^{2} w_{i j}}{\left(\alpha^{2} \nu+4 x i_{i j}^{2}\right)}\left(\frac{1+x_{i j}^{2}}{\alpha^{2}}\right)\right]\right\} \mathrm{d} x_{i j} .
\end{aligned}
$$

A matriz de variância-covariância de $\mathbf{u}_{i}$ pode ser escrita na forma

$$
\operatorname{Cov}\left(\mathbf{u}_{i}\right)=\operatorname{Var}\left(\mathbf{u}_{i}\right)^{1 / 2} \operatorname{Corr}\left(\mathbf{u}_{i}\right) \operatorname{Var}\left(\mathbf{u}_{i}\right)^{1 / 2}
$$

em que $\mathbf{R}\left(\mathbf{u}_{i}\right)$ representa a matriz de correlação de $\mathbf{u}_{i}$ e $\operatorname{Var}\left(\mathbf{u}_{i}\right)$ é dada por,

$$
\operatorname{Var}\left(\mathbf{u}_{i}\right)=\operatorname{diag}\left\{\operatorname{Var}\left(u_{i 1}\right), \ldots, \operatorname{Var}\left(u_{i s}\right)\right\}=\operatorname{diag}\left\{\mathrm{E}\left(u_{i 1}^{2}\right), \ldots, \mathrm{E}\left(u_{i s}^{2}\right)\right\}
$$

em que 


$$
\begin{aligned}
\operatorname{Var}\left(u_{i j}\right)=\mathrm{E}\left(u_{i j}^{2}\right)=\int_{-\infty}^{\infty}\{ & {\left[\frac{2}{\alpha} \mathrm{c}(\nu)\left\{1+\frac{4}{\nu}\left(\frac{x_{i j}}{\alpha}\right)^{2}\right\}^{-\left(\frac{\nu+1}{2}\right)} \frac{x_{i j}^{2}}{\alpha^{2}}\right] \times } \\
& {\left.\left[\frac{\alpha^{2}}{4\left(1+x_{i j}^{2}\right)}-2 w_{i j}+4 w_{i j}^{2}\left(\frac{1+x_{i j}^{2}}{\alpha^{2}}\right)\right]\right\} \mathrm{d} x_{i j} . }
\end{aligned}
$$

Denotamos, $\boldsymbol{\Sigma}_{i}=\operatorname{diag}\left\{\mathrm{E}\left(u_{i 1}^{2}\right), \ldots, \mathrm{E}\left(u_{i s}^{2}\right)\right\} \cdot \operatorname{Logo}$,

$$
\operatorname{Cov}\left(\mathbf{u}_{i}\right)=\boldsymbol{\Sigma}_{i}^{1 / 2} \operatorname{Corr}\left(\mathbf{u}_{i}\right) \boldsymbol{\Sigma}_{i}^{1 / 2}
$$

Portanto,

$$
\mathbf{\Psi}_{n}^{*}(\boldsymbol{\beta})=\sum_{i=1}^{n} \mathbf{X}_{i}^{\top} \mathbf{N}_{i} \operatorname{Cov}_{i}\left(\mathbf{u}_{i}\right)^{-1} \mathbf{u}_{i}
$$

Na prática a verdadeira matriz de correlação é desconhecida. Pela proposta de Liang e Zeger (1986), assumimos a existência do vetor $\rho(l \times 1)$ que caracteriza $\operatorname{Corr}\left(\mathbf{u}_{i}\right)$. Logo, a função de estimação de $\boldsymbol{\beta}$ fica dada por,

$$
\Psi_{n}(\boldsymbol{\beta})=\sum_{i=1}^{n} \mathbf{X}_{i}^{\top} \mathbf{N}_{i} \mathbf{C}_{i}^{-1} \mathbf{u}_{i}=\sum_{i=1}^{n} \mathbf{X}_{i}^{\top} \mathbf{W}_{\mathbf{i}} \mathbf{N}_{i}^{-1} \mathbf{u}_{i}
$$

em que $\mathbf{C}_{i}=\boldsymbol{\Sigma}_{i}^{1 / 2} \mathbf{R}(\rho) \boldsymbol{\Sigma}_{i}^{1 / 2}, \mathbf{W}_{i}=\mathbf{N}_{i} \mathbf{C}_{i}^{-1} \mathbf{N}_{i}$ e $\hat{\boldsymbol{\beta}}$ denota a raiz de (3.5).

\subsubsection{Estimação}

Para encontrarmos o estimador $\hat{\boldsymbol{\beta}}$ de $\boldsymbol{\beta}$ (fixados $\alpha$ e $\rho$ ) devemos resolver a seguinte equação de estimação:

$$
\Psi_{n}(\hat{\boldsymbol{\beta}})=0
$$

ou equivalentemente,

$$
\sum_{i=1}^{n} \mathbf{X}_{i}^{\top} \mathbf{W}_{\mathbf{i}} \mathbf{N}_{i}^{-1} \mathbf{u}_{i}=0
$$

Aplicando-se o método de Newton-Raphson chegou-se ao seguinte processo iterativo:

$$
\begin{aligned}
\boldsymbol{\beta}^{(m+1)}= & \boldsymbol{\beta}^{(m)}-\left\{\mathrm{E}\left[\frac{\partial}{\partial \boldsymbol{\beta}^{\top}} \boldsymbol{\psi}\left(\boldsymbol{\beta}^{(m)}\right)\right]\right\}^{-1} \boldsymbol{\psi}\left(\boldsymbol{\beta}^{(m)}\right) \\
= & \boldsymbol{\beta}^{(m)}-\left\{\left[\left(\sum_{i=1}^{n} \mathbf{X}_{i}^{\top} \hat{\mathbf{N}}_{i} \hat{\boldsymbol{\Sigma}}_{i}^{-1 / 2} \hat{\mathbf{R}}(\hat{\rho})^{-1} \hat{\boldsymbol{\Sigma}}_{i}^{-1 / 2} \hat{\mathbf{N}}_{i} \mathbf{X}_{i}\right)^{(m)}\right]^{-1}\right. \\
& {\left.\left[\sum_{i=1}^{n} \mathbf{X}_{i}^{\top} \hat{\mathbf{N}}_{i} \mathbf{W}_{i}^{-1}(\hat{\rho}) \hat{\mathbf{u}}_{i}\right]^{(m)}\right\} }
\end{aligned}
$$


sendo $m=0,1,2, \ldots$ o número de iterações. É importante ressaltar que as estimativas $\hat{\alpha}$ e $\hat{\rho}$ são fornecidas inicialmente e modificadas separadamente a cada passo do processo iterativo.

\section{Estimação de $\alpha$}

Sob a hipótese de independência entre as observações de um mesmo indivíduo e dado $\boldsymbol{\beta}$, a função escore do parâmetro $\alpha$ fica dada por

$$
\mathrm{U}_{\alpha}=\sum_{i=1}^{n} \sum_{j=1}^{s}\left[-\frac{1}{\alpha}+\frac{(\nu+1)\left\{4 \xi_{i j 2}^{2}\right\}}{\alpha\left\{\nu+4 \xi_{i j 2}^{2}\right\}}\right]
$$

e o estimador de $\alpha$ é a solução da equação $\mathrm{U}_{\hat{\alpha}}=0$.

Para estimar $\rho$, procedemos como descrito na Seção 2.2.2.

\subsubsection{Processo iterativo}

O processo iterativo é análogo ao processo iterativo derivado no Capítulo 2, com as respectivas mudanças nas quantidades envolvidas.

\subsubsection{Inferência}

A parte inferencial segue de forma análoga à Seção 2.2.3, considerando $\mathbf{u}_{i}, \mathbf{N}_{i}$ e $\mathbf{W}_{i}$ determinados, respectivamente, por (3.1), (3.2), (3.3) e considerando o estimador de máxima verossimilhança de $\alpha$ como solução de 3.7. Os cálculos para a obtenção das matrizes $\mathbf{S}_{i}(\boldsymbol{\beta})$ e $\mathbf{V}_{i}(\boldsymbol{\beta})$ são apresentados no Apêndice D.2.

\subsubsection{Estudo de simulação}

Com a finalidade de analisar o desempenho dos estimadores obtidos a partir do método de EEGs para modelos log-BS- $t$ conduzimos um estudo de simulação. Iremos assumir que $\mathbf{y}_{i}=$ $\left(y_{i 1}, \ldots, y_{i s}\right)^{\top}$ segue distribuição multivariada log-BS- $t$, como descrito em Kundu et al. (2013)), com vetor parâmetro de localização $\boldsymbol{\mu}_{i}=\left(\mu_{i 1}, \ldots, \mu_{i s}\right)^{\top}$, parâmetro de forma $\alpha, \nu$ graus de liberdade (que estamos supondo conhecido) e correlação $\rho$, para $i=1, \ldots, n$. Em adição, os componentes de $\boldsymbol{\mu}_{i}$ são modelados como

$$
\mu_{i j}=\beta_{0}+\beta_{1} x_{i j}
$$

em que $x_{i j}$ 's denotam valores fixos gerados de uma distribuição uniforme no intervalo $[0,1]$, para $i=1, \ldots, n$ e $j=1, \ldots, s$. Como assumido no caso da log-BS-EEG clássica consideramos estruturas de correlação, autorregressiva de primeira ordem e permutável, $\beta_{0}=4, \beta_{1}=-2, \alpha=0.5$ e $\rho=0.3,0.6$ e 0.9 , tamanhos amostrais $n=10,20,50$ e 80 e $s=3,5$ e 10 . Fixamos graus de liberdade $\nu=4,7,10$ e 30. Obtivemos o viés relativo (VR) e o erro quadrático médio (EQM) dos estimadores $\hat{\beta}_{0}, \hat{\beta}_{1}$ e $\hat{\alpha}$. Consideramos para cada cenário $R=5000$ réplicas.

Os resultados deste estudo de simulação encontram-se no Apêndice E. Em relação ao VR, observamos que o estimador de $\alpha$ é viesado e que à medida que o tamanho do grupo aumenta o viés diminui. De forma oposta, à medida que o coeficiente de correlação aumenta, o viés relativo aumenta, em valor absoluto. Já com relação aos estimadores dos $\beta^{\prime}$ s, os vieses são bem pequenos. 
Sob o ponto de vista de EQM, as estimativas dos parâmetros $\alpha, \beta_{0}$ e $\beta_{1}$ "melhoram"quanto maior a amostra, ou o grupo, o grau de liberdade. Observamos inclusive que quando a suposição da matriz de correlação está correta, quanto maior o grau de liberdade mais próximo fica $\hat{\rho}$ do $\rho$ utilizado para gerar os dados. É válido ressaltar que o EQM de $\hat{\alpha}$ não tem um padrão únido quando $\rho$ aumenta.

\subsubsection{Métodos de diagnóstico}

O procedimento de métodos de diagnóstico segue de forma análoga à Seção 2.2.5 considerando agora $\mathbf{u}_{i}, \mathbf{N}_{i}$ e $\mathbf{W}_{i}$ dados, respectivamente, por (3.1), (3.2), (3.3) e considerando o estimador de máxima verossimilhança de $\alpha$ como solução de (3.7). É necessário, porém, que sejam realizadas adaptações para a obtenção da influência local. Portanto, apresentaremos aqui apenas a matriz $\Delta$ para a influência local para os esquemas de perturbação da variável resposta e perturbação individual das covariáveis. No caso particular da ponderação de casos, assim como para os resíduos e distância de Cook, a forma matricial coincide com o caso clássico, necessitando apenas fazer as substituições adequadas de $\mathbf{u}_{i}, \mathbf{N}_{i}, \mathbf{W}_{i}$.

i) Perturbação da variável resposta

Quando consideramos a perturbação da variável resposta

$$
y_{\omega i j}=y_{i j}+\omega_{i j} s_{y_{i j}}
$$

em que $\omega_{i j}=0$ denota ausência da perturbação e $s_{y_{i j}}=\sqrt{\operatorname{Var}\left(y_{i j}\right)}$. Temos que

$$
\Psi(\boldsymbol{\beta} \mid \boldsymbol{\omega})=\mathbf{X}^{\top} \mathbf{W} \mathbf{N}^{-1} \mathbf{u}_{\omega}
$$

Então,

$$
\boldsymbol{\Delta}=\frac{\partial \boldsymbol{\Psi}(\boldsymbol{\beta} \mid \boldsymbol{\omega})}{\partial \boldsymbol{\omega}^{\top}}=\mathbf{X}^{\top} \mathbf{W} \mathbf{N}^{-1} \frac{\partial \mathbf{u}_{\omega}}{\partial \boldsymbol{\omega}^{\top}}
$$

avaliado em $\boldsymbol{\beta}=\hat{\boldsymbol{\beta}}$ e $\boldsymbol{\omega}=\boldsymbol{\omega}_{0}$. Definimos a forma matricial, $\frac{\partial \mathbf{u}}{\partial \boldsymbol{\omega}}=\dot{\mathcal{U}} \operatorname{com} \dot{\mathcal{U}}=\operatorname{diag}\left\{\dot{\mathcal{U}}_{1}, \ldots, \dot{\mathcal{U}}_{n}\right\}$, em que $\dot{\mathcal{U}}_{i}=\operatorname{diag}\left\{\frac{\partial u_{\omega i 1}}{\partial \omega_{i 1}}, \ldots, \frac{\partial u_{\omega i s}}{\partial \omega_{i s}}\right\}$.

$\mathrm{O}$ vetor de perturbação da variável resposta para a $i$-ésima unidade experimental fica dada por $\mathbf{u}_{\omega i}=\left(u_{\omega i 1}, \ldots, u_{\omega i s}\right)^{\top} \mathrm{com}$

$$
u_{\omega i j}=-\xi_{\omega i j 2}\left(\frac{1}{2 \xi_{\omega i j 1}}-\frac{2(\nu+1) \xi_{\omega i j 1}}{\nu+4 \xi_{\omega i j 2}^{2}}\right)
$$

em que $\xi_{\omega i j 1}=\frac{1}{\alpha} \cosh \left(\frac{y_{\omega i j}-\mu_{i j}}{2}\right)$ e $\xi_{\omega i j 2}=\frac{1}{\alpha} \operatorname{senh}\left(\frac{y_{\omega i j}-\mu_{i j}}{2}\right)$ e cuja derivada em relação a $\omega_{i j}$ é dada por,

$$
\begin{aligned}
\frac{\partial u_{\omega i j}}{\partial \omega_{i j}}= & -s_{y i j}\left[\frac{1}{4+4 \xi_{\omega i j 2}^{2} \alpha^{2}}-\left(\frac{\nu+1}{\nu+4 \xi_{\omega i j 2}^{2}}\right)\left(\frac{1}{\alpha^{2}}+2 \xi_{\omega i j 2}^{2}\right)+8 \xi_{\omega i j 2}^{2}\left(\frac{\nu+1}{\nu+4 \xi_{\omega i j 2}^{2}}\right)\right. \\
& \left.\left(\frac{1}{\alpha^{2}}+\xi_{\omega i j 2}^{2}\right) \frac{1}{\left(\nu+4 \xi_{\omega i j 2}^{2}\right)}\right] .
\end{aligned}
$$


iii) Perturbação em uma covariável

Consideramos o esquema de perturbação aditiva para $k$-ésima coluna da matriz $\mathbf{X}$, dada em (2.18). Então, precisamos apenas obter as derivadas de $\mathbf{N}_{\omega}, \mathbf{C}_{\omega}$ e $\mathbf{u}_{\omega}$ em relação a $\boldsymbol{\omega}^{\top}$. Temos que,

$$
\frac{\partial \mathbf{N}_{\omega}}{\partial \omega_{i j}}=\frac{\partial \mathbf{C}_{\omega}}{\partial \omega_{i j}}=0
$$

O vetor de perturbação da variável resposta para a $i$-ésima unidade experimental fica dada por $\mathbf{u}_{\omega i}=\left(u_{\omega i 1}, \ldots, u_{\omega i s}\right)^{\top} \mathrm{com}$

$$
u_{\omega i j}=-\xi_{\omega i j 2}\left(\frac{1}{2 \xi_{\omega i j 1}}-\frac{2(\nu+1) \xi_{\omega i j 1}}{\nu+4 \xi_{\omega i j 2}^{2}}\right),
$$

em que $\xi_{\omega i j 1}=\frac{1}{\alpha} \cosh \left(\frac{y_{i j}-\mu_{\omega i j}}{2}\right)$ e $\xi_{\omega i j 2}=\frac{1}{\alpha} \operatorname{senh}\left(\frac{y_{i j}-\mu_{\omega i j}}{2}\right)$ e cuja derivada em relação a $\omega_{i j}$ fica dada por,

$$
\begin{aligned}
\frac{\partial u_{\omega i j}}{\partial \omega_{i j}}= & \beta_{k} s_{x k}\left[\frac{1}{4+4 \xi_{\omega i j 2}^{2} \alpha^{2}}-\left(\frac{\nu+1}{\nu+4 \xi_{\omega i j 2}^{2}}\right)\left(\frac{1}{\alpha^{2}}+2 \xi_{\omega i j 2}^{2}\right)+8 \xi_{\omega i j 2}^{2}\left(\frac{\nu+1}{\nu+4 \xi_{\omega i j 2}^{2}}\right)\right. \\
& \left.\left(\frac{1}{\alpha^{2}}+\xi_{\omega i j 2}^{2}\right) \frac{1}{\left(\nu+4 \xi_{\omega i j 2}^{2}\right)}\right] .
\end{aligned}
$$

\subsubsection{Aplicação}

Vamos considerar nesta seção um conjunto de dados referente a um estudo realizado na Escola de Educação Física e Esporte da Universidade de São Paulo (USP) no primeiro semestre 2016, em que 70 corredores foram avaliados sob as condições calçado e descalço em corridas em esteiras em três períodos. Os voluntários foram divididos em dois grupos de 35 indivíduos cada: o grupo controle e o grupo experimental. No período inicial não havia ainda treinamento. O grupo experimental ficou mais tempo treinando descalço do que o grupo o controle, mas ambos foram avaliados nas duas condições "calçado"e "descalço". Para cada indivíduo foi obtido o valor máximo do primeiro pico de força vertical (em Newtons) em duas condições diferentes durante a corrida na esteira: calçado ou descalço e em três ocasiões: início, 3 meses após o início do experimento ou 6 meses após o início do experimento. A saber, o experimento conta com um conjunto de dados desbalanceado, isto é, os indivíduos possuem quantidades diferentes de observações.

O interesse principal do experimento é determinar se a condição calçado reduz a força vertical inicial ( $f_{Y} 1$ ). Espera-se que o grupo experimental tenha um desempenho melhor do que o grupo controle pois há na literatura teorias que dizem que correr descalço deve levar a um desempenho melhor do que correr calçado e o grupo experimental treinou mais tempo descalço.

Podemos observar pelo gráfico da densidade na Figura 3.1 (à esquerda), um comportamento simétrico da variável de interesse fyl. Observamos também na Figura 3.1 (à direita), os boxplots da variável $f_{Y} 1$ segundo a condição (calçado ou descaldo) para todas as semanas e todos os grupos. Nota-se valores menores de $\mathrm{f}_{\mathrm{Y}} 1$ sob condição descalço do que sob condição calçado.

Na Tabela 3.1 descrevemos os valores médios com os respectivos desvios padrão e coeficientes de variação para $\mathrm{fy}_{\mathrm{y}} \mathrm{p}$ para cada grupo (experimental ou controle) e por período de tempo para 

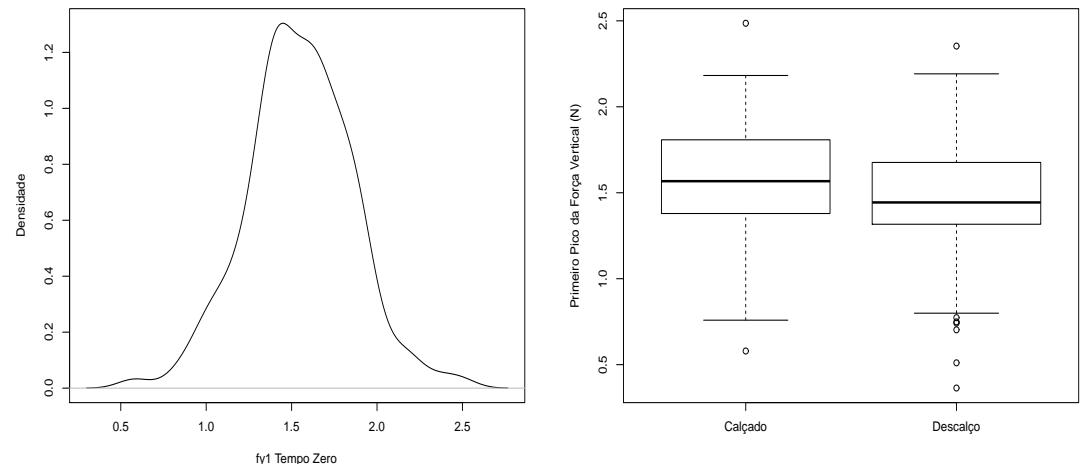

Figura 3.1: Densidade da variável fy1 (à esquerda) e boxplot da variável fy1 segundo a condição (calçado ou descaldo) para todas as semanas e todos os grupos (à direita).

todas as condições (calçado ou descalço). Nota-se um decréscimo no valor médio de fy 1 após 3 meses para os dois grupos, sendo a redução mais acentuada para o grupo experimental . Nota-se também um aumento da variabilidade. Já ápós seis meses, enquanto praticamente não há alteração na média do primeiro pico de força vertical do grupo experimental, nota-se um aumento de $f_{y} 1$ no grupo controle. Pela Figura 3.2 pode-se perceber melhor as diferenças no comportamento da variável fy 1 nos grupos controle e experimental. Vamos também investigar uma possível interação entre as variáveis grupo e momento.

Tabela 3.1: Medidas-resumo para o primeiro pico de força vertical (fy1) segundo o grupo e o momento para todas as condições.

\begin{tabular}{ccccc}
\hline & & Início & 3 Meses & 6 Meses \\
\hline \multirow{3}{*}{ Grupo Controle } & Média & 1,5356 & 1,4876 & 1,5555 \\
& Desvio padrão & $(0,3004)$ & $(0,2915)$ & $(0,3267)$ \\
& Coeficiente de Variação & 0,195 & 0,195 & 0,21 \\
& & $\mathrm{n}=67$ & $\mathrm{n}=33$ & $\mathrm{n}=28$ \\
\hline \multirow{3}{*}{ Grupo Experimental } & Média & 1,5594 & 1,3900 & 1,3942 \\
& Desvio padrão & $(0,3167)$ & $(0,39970)$ & $(0,4073)$ \\
& Coeficiente de Variação & 0,203 & 0,287 & 0,33 \\
& & $\mathrm{n}=68$ & $\mathrm{n}=39$ & $\mathrm{n}=30$ \\
\hline
\end{tabular}

Denotaremos por $\mathrm{y}_{i j k l}$ o valor máximo do primeiro pico de força vertical para o $i$-ésimo indivíduo $(i=1 \ldots, 70)$, no $j$-ésimo grupo ( $=1$ controle, $=2$ experimental $), k$-ésima ocasião $(=1$ início, $=2$ após 3 meses, $=3$ após 6 meses), para $\ell$-ésima condição ( $=1$ calçado, $=2$ descalço). A fim de compararmos as duas distribuições trabalhadas durante a tese vamos supor que $\mathrm{y}_{i j k l} \sim \log$-BS $\left(\alpha, \mu_{j k l}\right)$ e $\mathrm{y}_{i j k l} \sim$ $\log -\mathrm{BS}-t\left(\alpha, \mu_{j k l}\right)$, em que $\alpha>0$ é o parâmetro de forma, com parte sistemática dada por,

$$
\mu_{j k l}=\lambda+\beta_{j}+\gamma_{k}+\delta_{l}+(\beta \gamma)_{j k}
$$

para $j=1,2 ; k=1,2,3$ e $l=1,2$ com parametrização casela de referência, em que $(\beta \gamma)_{j k}$ representa a iteração entre o grupo e o momento e $\beta_{j}, \gamma_{k}$ e $\delta_{l}$ são os efeitos principais: grupo, 

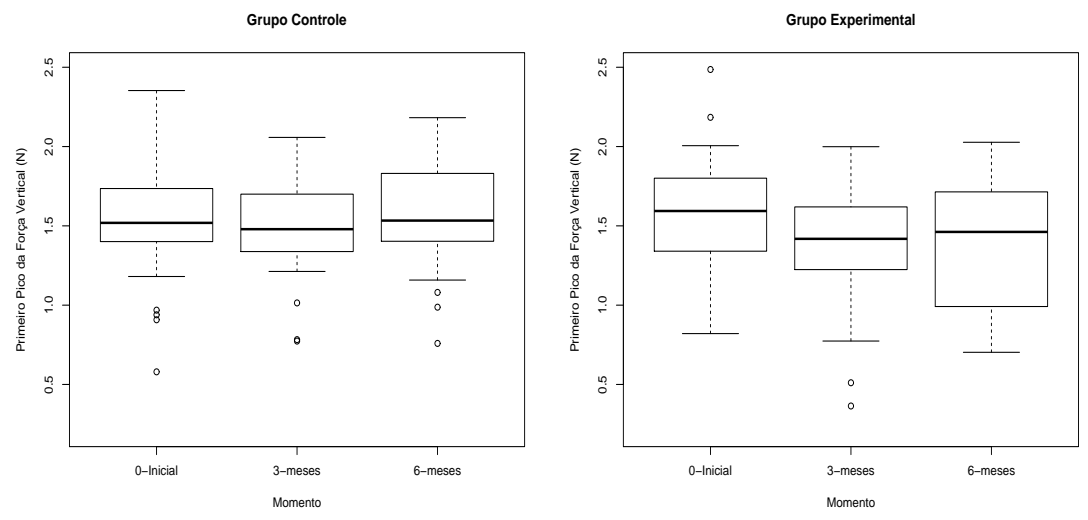

Figura 3.2: Boxplots (robusto) da variável fy1 no grupo controle (à esquerda) e boxplot (robusto) da variável fy1 do grupo experimental (à direita).

momento e condição, respectivamente. Teremos as restrições $\beta_{1}=0, \gamma_{1}=0, \delta_{1}=0,(\beta \gamma)_{1 k}=0$ e $(\beta \gamma)_{j 1}=0$. Propomos $\mathbf{R}_{i}=\mathbf{R}_{i}(\rho) \operatorname{com} R_{i j j^{\prime}}=1$, para $j=j^{\prime}$ e $R_{i j j^{\prime}}=\rho$ para $j \neq j^{\prime}$. Iremos denominar os modelos log-BS e log-BS- $t$ com estrutura de correlação permutável, respectivamente, como log-BS-EEG e log-BS-t-EEG.

Primeiramente, vamos supor que as respostas seguem distribuição marginal $\log$-BS $\left(\mu_{j k l}, \alpha\right)$. As estimativas dos parâmetros são descritas na Tabela 3.2. Temos ainda que

Tabela 3.2: Estimativas dos parâmetros e erros padrão aproximados para o modelo log-BS-EEG ajustado aos dados sobre produtividade de capital público.

\begin{tabular}{crrrrrrrrr}
\hline & $\lambda$ & $\beta_{2}$ & $\gamma_{2}$ & $\gamma_{3}$ & $\delta_{2}$ & $(\beta \gamma)_{22}$ & $(\beta \gamma)_{23}$ & $\alpha$ & $\rho$ \\
\hline Estimativas & 1,591 & 0,010 & $-0,055$ & 0,001 & $-0,096$ & $-0,099$ & $-0,210$ & 0,247 & 0,459 \\
Erro Padrão & 0,045 & 0,065 & 0,073 & 0,052 & 0,034 & 0,1 & 0,082 & & \\
Valor p & $<0,01$ & & & & 0,01 & & 0,04 & & \\
\hline
\end{tabular}

A Figura 3.3 apresenta o gráfico da distância de Cook do modelo log-BS-EEG. Como podemos notar as observações \# $(15,1)$, \#(51,5), \# $(8,3)$, \#(6,4) e \#(56,6) são consideradas pontencialmente influentes. A Tabela 3.3 descreve as mudanças relativas (MR) das estimativas, após excluirmos os indivíduos com observações que são consideradas potencialmente influentes. Os valores $p$ para as novas estimativas estão entre parênteses. Podemos notar pela Tabela 3.3 que quando eliminamos o indivíduo 8 do conjunto de dados há mudanças inferenciais. A observação \# $(8,3)$ apresenta o menor fy1. A Figura 3.5 (à esquerda) apresenta o gráfico dos resíduos para o modelo log-BS ajustado via EEGs, no qual podemos perceber alguns resíduos bem altos. Podemos observar através da do gráfico de envelope na Figura 3.5 que a suposição da distribuição log-BS não parece ser adequada. Pelo comportamento do envelope, uma distribuição de caudas mais pesadas poderia ser mais indicada.

Vamos reanalisar esses dados considerando agora distribuições marginais log-BS-t $\left(\mu_{j k l}, \alpha, \nu\right)$ para as respostas. As estimativas dos parâmetros são descritas na Tabela 3.4.

Na Figura 3.6 detectamos dois casos como potencialmente influentes: \#(3,6) e \#(56,6), cuja a exclusão não causa mudança inferencial. Na Figura 3.8 (esquerda) podemos perceber que os resíduos padronizados se mostram de forma aleatória, não apresentando tendência e que, em geral, estão 


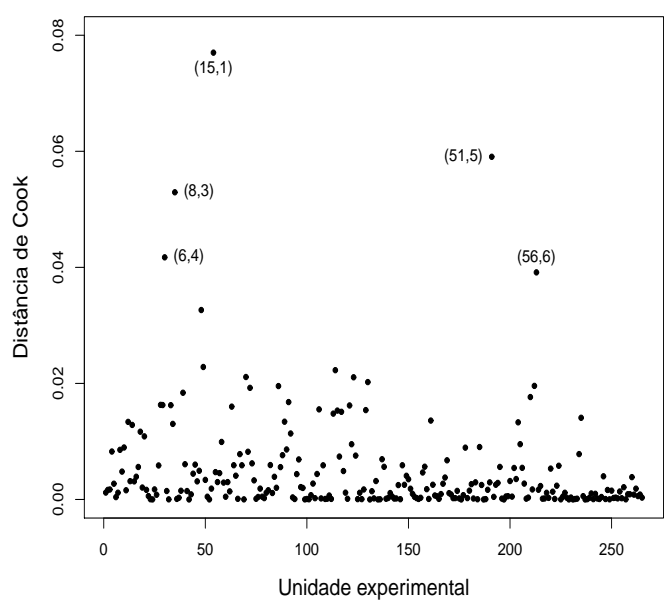

Figura 3.3: Gráfico de distância de Cook para o modelo log-BS ajustado via EEG aos dados sobre condições experimentais calçados e descalços.

Tabela 3.3: $M R$ (em \%) e correspondentes valores $p$ entre parênteses do modelo log-BS ajustados via EEG aos dados sobre condições experimentais calçados e descalços.

\begin{tabular}{crrrrrrrrr}
\hline $\begin{array}{c}\text { indivíduos } \\
\text { excluídas }\end{array}$ & $\lambda$ & $\beta_{2}$ & $\gamma_{2}$ & $\gamma_{3}$ & $\delta_{2}$ & $(\beta \gamma)_{22}$ & $(\beta \gamma)_{23}$ & $\alpha$ & $\rho$ \\
\hline 15 & 1,200 & $-140,450$ & 67,200 & $-9160,390$ & 10,970 & $-38,160$ & $-20,410$ & $-1,360$ & 5,390 \\
& $<0,01$ & & & & $<0,01$ & & & & \\
\hline 51 & 0,452 & $-44,536$ & 18,717 & 7289,145 & 5,746 & $-10,638$ & 16,627 & $-0,418$ & 0,963 \\
& $<0,01$ & & & & $<0,01$ & & 0,01 & & \\
\hline 8 & $-0,220$ & 13,540 & $-0,060$ & $-103,990$ & $-7,900$ & $-45,260$ & $-8,110$ & $-2,270$ & 5,860 \\
& $<0,01$ & & & & 0,010 & & 0,06 & & \\
\hline 6 & $-0,480$ & 71,490 & $-0,280$ & $-94,680$ & $-16,520$ & $-3,530$ & 0,230 & $-1,680$ & 5,280 \\
& $<0,01$ & & & & 0,010 & & 0,04 & & \\
\hline 56 & $-0,350$ & $-7,180$ & $-0,230$ & $-40,860$ & $-11,870$ & 20,360 & $-2,150$ & $-0,640$ & 2,130 \\
& $<0,01$ & & & & 0,01 & & 0,05 & & \\
\hline
\end{tabular}

no intervalo [-2,2], apresentando alguns casos como pontos aberrantes. Comparando a Figura 3.8 (direita) com a Figura 3.5 (direita) verificamos que o modelo log-BS-t-EEG parece mais adequado para análise dos dados estudados.

Portanto, é razoável assumir que marginalmente $\mathrm{y}_{i j} \sim \log$-BS- $t-\operatorname{EEG}\left(\alpha, \mu_{j k l}\right)$, com $\mu_{j k l}=$ $\lambda+\beta_{2}+\gamma_{2}+\gamma_{3}+\delta_{2}+(\beta \gamma)_{22}+(\beta \gamma)_{23}$ denotando a média do pico de força inicial máximo. Portanto, das estimativas dadas na Tabela 3.4, podemos concluir que o pico de força diminui quando a condição do corredor é calçado e podemos confirmar a iteração entre o grupo experimental e o momento. Havendo diminuição do pico de força no grupo experimental conforme o tempo passa.

A Figura 3.9 mostra que quando os dados são ajustados considerando log-BS-t-EEG são atribuídos pesos menores para dados com resíduos maiores. Observe que os pontos marcados no gráfico, que possuem resíduos maiores e pesos menores, são justamente os pontos destacados na análise $\log$-BS-EEG. 

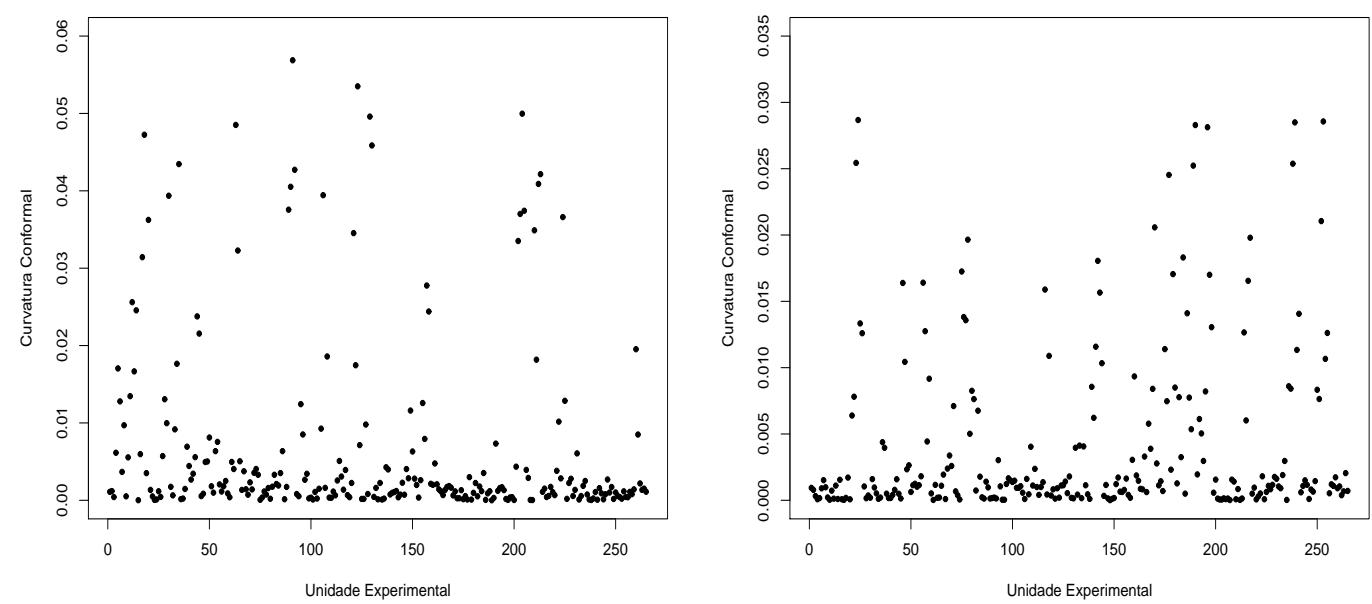

Figura 3.4: Gráficos de influência local conformal sob os esquemas: ponderação de casos para o modelo

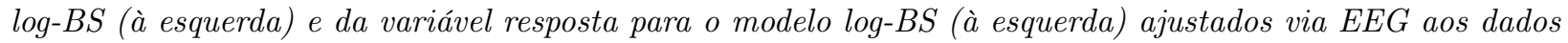
sobre condições experimentais calçados e descalços.

Tabela 3.4: Estimativas do modelo log-BS-t ajustados via EEGs dos dados sobre condições experimentais calçados e descalços.

\begin{tabular}{rrrrrrrrrr}
\hline \hline Parâmetro & $\lambda$ & $\beta_{2}$ & $\gamma_{2}$ & $\gamma_{3}$ & $\delta_{2}$ & $(\beta \gamma)_{22}$ & $(\beta \gamma)_{23}$ & $\alpha$ & $\rho$ \\
\hline Estimativa & 1,596 & 0,025 & $-0,033$ & 0,026 & $-0,114$ & $-0,102$ & $-0,171$ & 0,333 & 0,490 \\
Erro Padrão & 0,045 & 0,065 & 0,073 & 0,052 & 0,034 & 0,100 & 0,082 & & \\
valor p & $<0,01$ & & & & $<0,01$ & & 0,04 & & \\
\hline
\end{tabular}
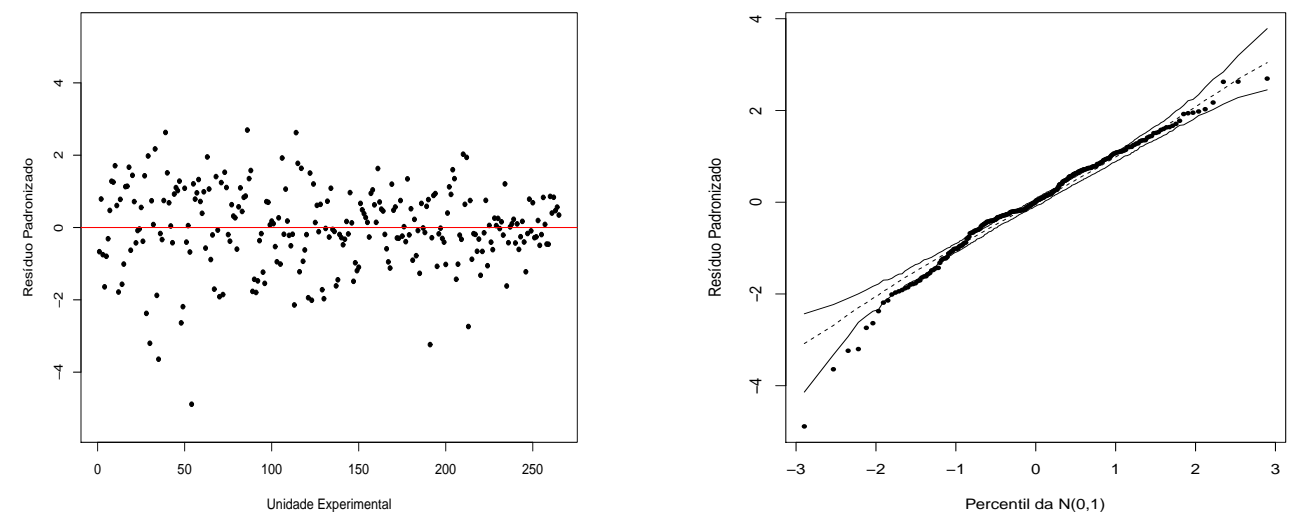

Figura 3.5: Gráfico de resíduos do modelo log-BS ajustado via EEGs (à esquerda) e gráfico normal de probabilidades para o resíduo de Pearson padronizado referente ao ajuste do modelo log-BS (à direita) ajustados via EEG aos dados sobre condições experimentais calçados e descalços. 


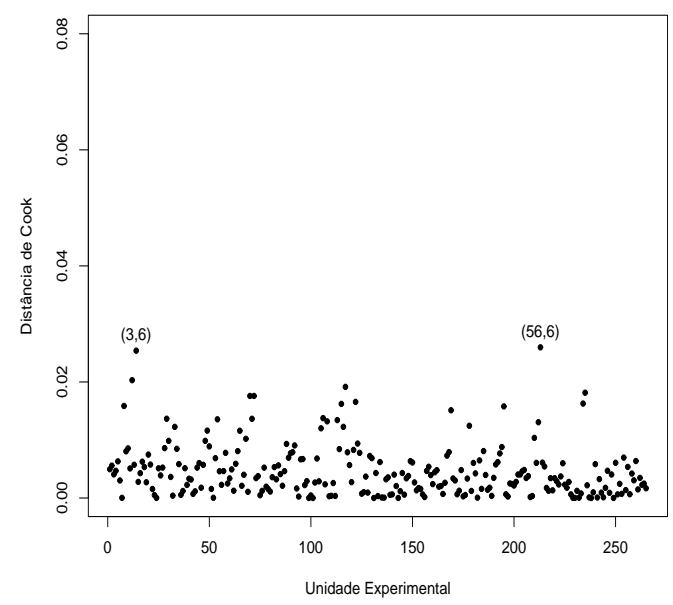

Figura 3.6: Gráfico de distância de Cook para o modelo log-BS-t ajustado via EEG aos dados sobre condições experimentais calçados e descalços.
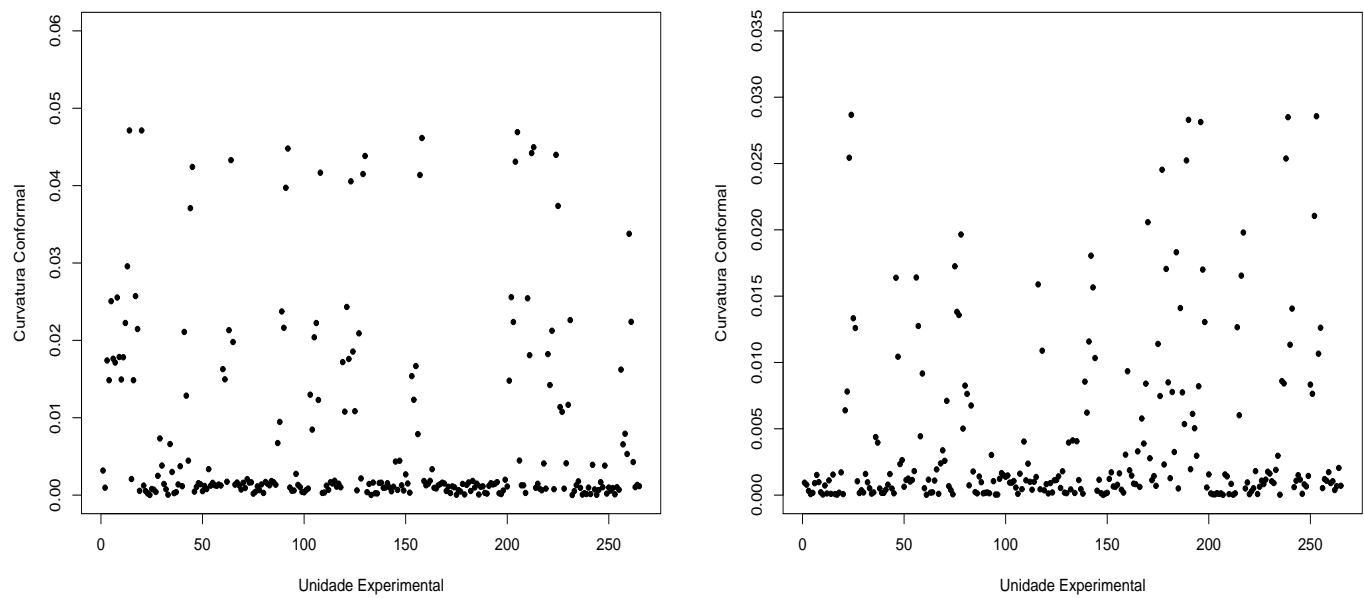

Figura 3.7: Gráficos de influência local conformal sob os esquemas: ponderação de casos para o modelo log-BS-t (à esquerda) e da variável resposta para o modelo log-BS-t (à esquerda) ajustados via EEG aos dados sobre condições experimentais calçados e descalços. 

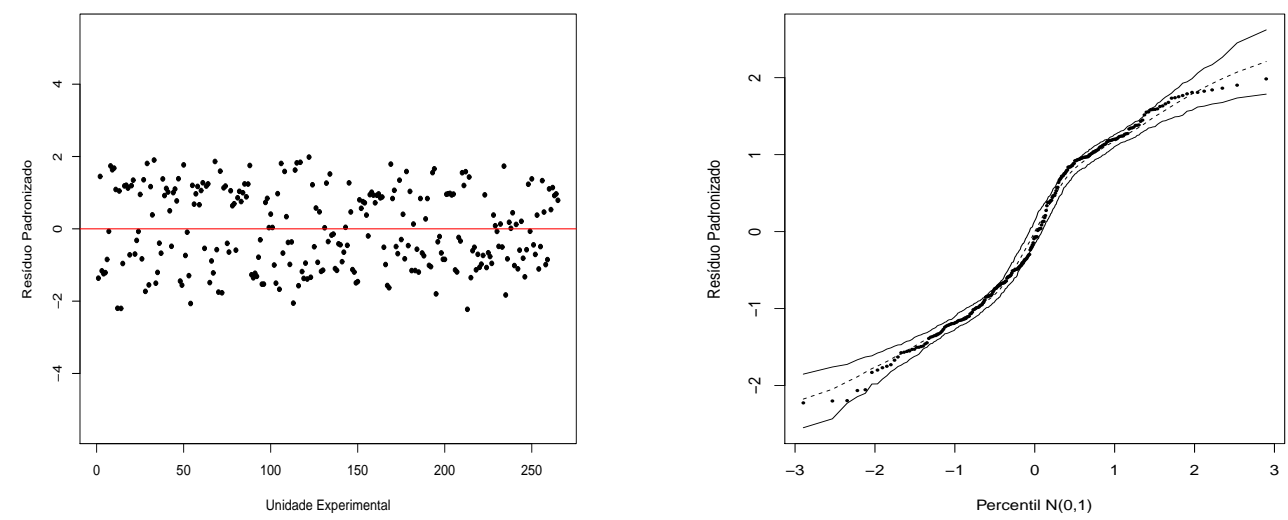

Figura 3.8: Gráfico de resíduos do modelo log-BS-t ajustado via EEGs (à esquerda) e gráfico normal de probabilidades para o resíduo de Pearson padronizado referente ao ajuste do modelo log-BS-t (à direita) ajustados via EEG aos dados sobre condições experimentais calçados e descalços.

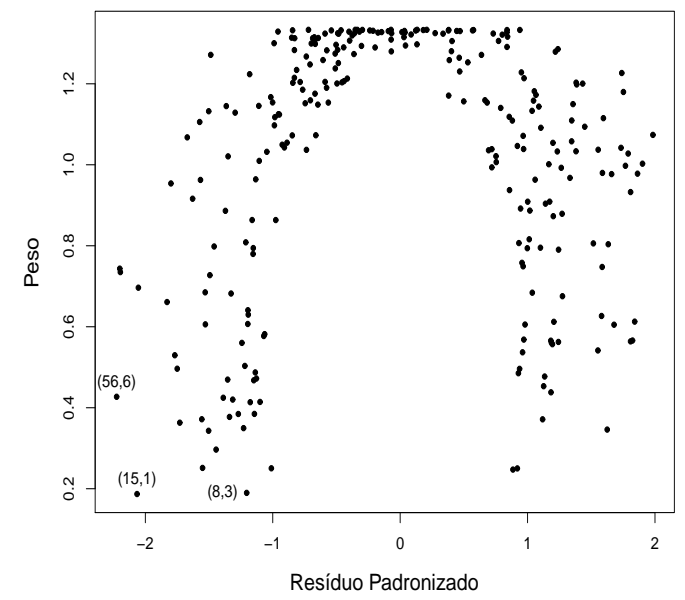

Figura 3.9: Pesos versus resíduos padronizados referente ao modelo log-BS-t ajustados via EEGs dos dados sobre condições experimentais calçados e descalços. 


\section{Capítulo 4}

\section{Equações de Estimação Conjuntas para a Média e Forma}

Rieck e Nedelman (1991) formularam um modelo log-linear para a distribuição BS. Com o intuito de fornecer uma estrutura estatística adequada para o problema da estimação, os autores assumiram que o parâmetro de forma $\alpha$ é independente do trabalho por ciclo. Embora, Rieck e Nedelman (1991) aleguem que essa hipótese é aparentemente válida para os dados de fadiga investigados em seu artigo, alertaram para o fato que nem sempre essa hipótese é justificada. Da mesma forma, Galea et al. (2004) também mencionam o problema de supor homogeneidade do parâmetro de forma no modelo log-BS.

Diante desse dilema, Xie e Wei (2007) propuseram testes para detectar homogeneidade do parâmetro de forma e apresentaram estatísticas de razão de verossimilhanças e escore. Qu e Xie (2011) investigaram o teste escore para homogeneidade para o parâmetro $\alpha$ do modelo log-BS quando os dados são censurados.

Li et al. (2012) introduziram então uma classe de modelos de regressão log-BS heterogêneo e obtiveram estimadores de máxima verossimilhança para os parâmetros e apresentaram medidas de diagnóstico baseadas em influência local e método de deleção de casos. Neste capítulo estudaremos o modelo de regressão log-BS heterogêneo sob o ponto de vista de equação de estimação e faremos as devidas extensões para o modelo log-BS-t.

\subsection{Equação de estimação conjunta para o modelo log-BS.}

\subsubsection{Derivação de função de estimação conjunta}

Consideramos agora uma amostra aleatória de $n$ unidades amostrais, $\mathbf{t}_{1}, \ldots, \mathbf{t}_{n}$, em que $\mathbf{t}_{i}=$ $\left(\mathrm{t}_{i 1}, \ldots, \mathrm{t}_{i s_{i}}\right)^{\top}$ e admitimos que cada componente $\mathrm{t}_{i j} \sim \mathrm{BS}\left(\alpha_{i j}, \beta_{i j}^{*}, 2\right)$, de forma que cada componente $\mathrm{y}_{i j}=\log \left(\mathrm{t}_{i j}\right) \sim \mathrm{SN}\left(\alpha_{i j}, \mu_{i j}, 2\right)$, isto é, sua distribuição marginal é dada por

$$
f\left(y_{i j}\right)=\frac{1}{\alpha_{i j} \sqrt{2 \pi}} \cosh \left(\frac{y_{i j}-\mu_{i j}}{2}\right) \exp \left\{-2\left[\frac{1}{\alpha_{i j}} \operatorname{senh}\left(\frac{y_{i j}-\mu_{i j}}{2}\right)\right]^{2}\right\}, y_{i j} \in \mathbb{R},
$$


$\alpha_{i j}>0, i=1, \ldots, n, j=1, \ldots, s_{i}$. Ou seja, vamos incorporar a heterogeneidade do parâmetro de forma ao que foi feito no Capítulo 2. Sem perda de generalidade, assumiremos experimentos balanceados $s_{i}=s$.

O parâmetro de localização é expresso na forma

$$
\mu_{i j}=\eta_{i j}=\mathbf{x}_{i j}^{\top} \boldsymbol{\beta}
$$

adicionalmente, o parâmetro de forma agora assume a forma,

$$
g\left(\alpha_{i j}\right)=\delta_{i j}=\mathbf{z}_{i j}^{\top} \gamma
$$

em que $\boldsymbol{\beta}=\left(\beta_{1}, \ldots \beta_{p}\right)^{\top}$ e $\boldsymbol{\gamma}=\left(\gamma_{1}, \ldots \gamma_{q}\right)^{\top}$ são, respectivamente, vetore $p$ e $q$-dimensionais de parâmetros desconhecidos, $\mathbf{x}_{i j}=\left(x_{i j 1} \ldots x_{i j p}\right)^{\top}$ e $\mathbf{z}_{i j}=\left(z_{i j 1} \ldots z_{i j q}\right)^{\top}$ contém valores de variávéis explicativas associadas a $\mu_{i j}$ e $\alpha_{i j}$, respectivamente, e $g(\cdot)$ é uma função de ligação monótona e duas vezes diferenciável. Devido à bimodalidade da função densidade da SN quando $\alpha>2$, que pode causar múltiplos máximos na função de verossimilhança, é razoável impor que $\alpha_{i j} \leq 2$. A exemplo do que é feito em Li et al. (2012) consideramos a função de ligação logarítmica, ou seja,

$$
\log \left(\alpha_{i j}\right)=\mathbf{z}_{i j}^{\top} \gamma \text { ou } \quad \alpha_{i j}=\exp \left(\mathbf{z}_{i j}^{\top} \gamma\right)
$$

Utilizaremos a definição de função de estimação linear ótima dada em (1.19). Baseados na função $\mathbf{u}_{i}$ definida na Seção 2.2.1 são construídas as equações de estimação para estimar a média (mas agora abandonaremos a suposição que $\alpha_{i j}=\alpha$ ). Para estimar o vetor de parâmetro de forma, $\boldsymbol{\alpha}$, será usado a função escore de $\boldsymbol{\alpha}$. O logaritmo da função de verossimilhança em função de $\alpha_{i j}$ fica dado por

$$
l\left(\alpha_{i j} ; y_{i j}\right)=\frac{1}{2} \log (2 \pi)+\log \left(\xi_{i j 1}\right)-2 \xi_{i j 2}^{2} .
$$

Observe que,

$$
\frac{\partial \xi_{i j 1}}{\partial \alpha_{i j}}=-\frac{1}{\alpha_{i j}^{2}} \cosh \left(\frac{y_{i j}-\mu_{i j}}{2}\right)=-\frac{1}{\alpha_{i j}} \xi_{i j 1}
$$

e

$$
\frac{\partial \xi_{i j 2}}{\partial \alpha_{i j}}=-\frac{1}{\alpha_{i j}^{2}} \operatorname{senh}\left(\frac{y_{i j}-\mu_{i j}}{2}\right)=-\frac{1}{\alpha_{i j}} \xi_{i j 2}
$$

Então,

$$
\begin{aligned}
\frac{\partial l\left(\mu_{i j} ; y_{i j}\right)}{\partial \mu_{i j}} & =\frac{1}{\xi_{i j 1}} \xi_{i j 1}\left(-\frac{1}{\alpha_{i j}}\right)-4 \xi_{i j 2} \xi_{i j 2}\left(-\frac{1}{\alpha_{i j}}\right) \\
& =-\frac{1}{\alpha_{i j}}+4 \xi_{i j 2}^{2}\left(\frac{1}{\alpha_{i j}}\right)=\frac{1}{\alpha_{i j}}\left(4 \xi_{i j 2}^{2}-1\right) .
\end{aligned}
$$


Portanto, a função escore do parâmetro $\alpha_{i j}$ fica dada por,

$$
v_{i j}=\frac{1}{\alpha_{i j}}\left[\frac{4}{\alpha_{i j}^{2}} \operatorname{senh}^{2}\left(\frac{y_{i j}-\mu_{i j}}{2}\right)-1\right],
$$

e definimos $\mathbf{v}_{i}=\left(v_{i 1}, \ldots, v_{i s}\right)^{\top}$. Análogo ao que foi feito no Apêndice A podemos mostrar que $\mathbf{v}_{i}$ são vetores com média zero.

Temos que

$$
\mathrm{E}\left(\frac{\partial \mathbf{v}_{i}}{\partial \boldsymbol{\gamma}^{\top}}\right)^{\top}=\mathrm{E}\left(\frac{\partial \mathbf{v}_{i}}{\partial \boldsymbol{\alpha}_{i}} \frac{\partial \boldsymbol{\alpha}_{i}}{\partial \boldsymbol{\delta}_{i}} \frac{\partial \boldsymbol{\delta}_{i}}{\partial \boldsymbol{\gamma}^{\top}}\right)^{\top}=\mathbf{Z}_{i}^{\mathrm{T}} \mathbf{G}_{i} \mathbf{M}_{i}
$$

em que $\mathbf{G}_{i}=\operatorname{diag}\left\{\partial g^{-1}\left(\mathbf{z}_{i 1}^{\top} \boldsymbol{\gamma}\right) / \partial \delta_{i 1}, \ldots, \partial g^{-1}\left(\mathbf{z}_{i s}^{\top} \boldsymbol{\gamma}\right) / \partial \delta_{i s}\right\}$ e $\mathbf{M}_{i}=\operatorname{diag}\left\{-\frac{2}{\alpha_{i 1}^{2}}, \ldots,-\frac{2}{\alpha_{i s}^{2}}\right\}$.

E temos que

$$
\operatorname{Cov}\left(\mathbf{v}_{i}\right)=\operatorname{Var}\left(\mathbf{v}_{i}\right)^{1 / 2} \operatorname{Corr}\left(\mathbf{v}_{i}\right) \operatorname{Var}\left(\mathbf{v}_{i}\right)^{1 / 2}=\boldsymbol{\Omega}_{i}^{1 / 2} \operatorname{Corr}\left(\mathbf{v}_{i}\right) \boldsymbol{\Omega}_{i}^{1 / 2},
$$

em que $\operatorname{Corr}\left(\mathbf{v}_{i}\right)$ é a verdadeira matriz de correlação entre as observações $v_{i 1}, \ldots, v_{i s}$ e $\boldsymbol{\Omega}_{i}=$ $\operatorname{diag}\left\{\frac{2}{\alpha_{i 1}^{2}}, \ldots, \frac{2}{\alpha_{i s}^{2}}\right\}$.

A função de estimação linear ótima para $\gamma$ fica, portanto, dada por,

$$
\boldsymbol{\zeta}_{n}(\gamma)=\sum_{i=1}^{n} \mathbf{Z}_{i}^{\top} \mathbf{G}_{i} \mathbf{M}_{i} \operatorname{Cov}\left(\mathbf{v}_{i}\right)^{-1} \mathbf{v}_{i}
$$

Os cálculos detalhados para a obtenção da equação (4.2) são apresentados no Apêndice D.

Seja $\boldsymbol{\theta}=\left(\boldsymbol{\beta}^{\top}, \boldsymbol{\gamma}^{\top}\right)^{\top}$ e consideremos $\mathbf{d}_{i}=\left(\mathbf{u}_{i}^{\top}, \mathbf{v}_{i}^{\top}\right)^{\top}$. Uma função de estimação ótima para $\boldsymbol{\theta}$ gerada por $\mathbf{d}_{i}$, é dada por,

$$
\boldsymbol{\Gamma}_{n}(\boldsymbol{\theta})^{0}=\mathrm{E} \sum_{i=1}^{n}\left(\begin{array}{cc}
\frac{\partial \mathbf{u}_{i}}{\partial \boldsymbol{\beta}^{\top}} & \frac{\partial \mathbf{u}_{i}}{\partial \boldsymbol{\gamma}^{\top}} \\
\frac{\partial \mathbf{v}_{i}}{\partial \boldsymbol{\beta}^{\top}} & \frac{\partial \mathbf{v}_{i}}{\partial \boldsymbol{\gamma}^{\top}}
\end{array}\right)^{\top} \operatorname{Cov}\left(\mathbf{d}_{i}\right)^{-1} \mathbf{d}_{i} .
$$

Do Apêndice D podemos ver que,

$$
\boldsymbol{\Gamma}_{n}(\boldsymbol{\theta})^{0}=\sum_{i=1}^{n}\left(\begin{array}{cc}
\mathbf{X}_{i}^{\top} \mathbf{N}_{i} & \mathbf{0} \\
\mathbf{0} & \mathbf{Z}_{i}^{\top} \mathbf{G}_{i} \mathbf{M}_{i}
\end{array}\right) \mathbf{A}_{i}^{-1} \mathbf{d}_{i},
$$

com,

$$
\begin{aligned}
\mathbf{A}_{i}=\operatorname{cov}\left(\mathbf{d}_{i}\right) & =\left(\begin{array}{cc}
\operatorname{Cov}\left(\mathbf{u}_{i}\right) & \operatorname{Cov}\left(\mathbf{u}_{i}, \mathbf{v}_{i}\right) \\
\operatorname{Cov}\left(\mathbf{u}_{i}, \mathbf{v}_{i}\right) & \operatorname{Cov}\left(\mathbf{v}_{i}\right)
\end{array}\right) \\
& =\left(\begin{array}{cc}
\boldsymbol{\Sigma}_{i}^{1 / 2} \operatorname{Corr}\left(\mathbf{u}_{i}\right) \boldsymbol{\Sigma}_{i}^{1 / 2}, & \operatorname{Cov}\left(\mathbf{u}_{i}, \mathbf{v}_{i}\right) \\
\operatorname{Cov}\left(\mathbf{u}_{i}, \mathbf{v}_{i}\right) & \boldsymbol{\Omega}_{i}^{1 / 2} \operatorname{Corr}\left(\mathbf{v}_{i}\right) \boldsymbol{\Omega}_{i}^{1 / 2}
\end{array}\right),
\end{aligned}
$$

em que $\mathbf{N}_{i}=\mathrm{E}\left(\frac{\partial \mathbf{u}_{i}}{\partial \boldsymbol{\mu}_{i}}\right)=\operatorname{diag}\left\{\mathrm{E}\left(\dot{u}_{i}\right), \ldots, \mathrm{E}\left(\dot{u}_{i s}\right)\right\}, \operatorname{com~} \mathrm{E}\left(\dot{u}_{i j}\right)=\frac{1}{4} \mathrm{E}\left\{\operatorname{sech}^{2}\left(\frac{y_{i 1}-\mu_{i 1}}{2}\right)\right\}-\frac{1}{2}-\frac{1}{\alpha_{i j}^{2}}$, $\operatorname{Corr}\left(\mathbf{u}_{i}\right)$ e $\operatorname{Corr}\left(\mathbf{v}_{i}\right)$ são as verdadeiras matrizes de correlação de $\mathbf{u}_{i}$ e $\mathbf{v}_{i}$ respectivamente, $\boldsymbol{\Sigma}_{i}=$ 
$\operatorname{diag}\left\{\operatorname{Var}\left(u_{i 1}\right), \ldots, \operatorname{Var}\left(u_{i s}\right)\right\}, \operatorname{com} \operatorname{Var}\left(u_{i j}\right)=\mathrm{E}\left\{\frac{1}{4} \operatorname{tgh}^{2}\left(\frac{y_{i j}-\mu_{i j}}{2}\right)\right\}+\frac{1}{4}+\frac{1}{\alpha_{i j}^{2}}$,

$\mathbf{G}_{i}=\operatorname{diag}\left(\partial g^{-1}\left(\mathbf{z}_{i 1}^{\top} \boldsymbol{\gamma}\right) / \partial \delta_{i 1}, \ldots, \partial g^{-1}\left(\mathbf{z}_{i s}^{\top} \gamma\right) / \partial \delta_{i s}\right), \mathbf{M}_{i}=\operatorname{diag}\left\{-\frac{2}{\alpha_{i 1}^{2}}, \ldots,-\frac{2}{\alpha_{i s}^{2}}\right\}$,

$\boldsymbol{\Omega}_{i}=\operatorname{diag}\left\{\frac{2}{\alpha_{i 1}^{2}}, \ldots, \frac{2}{\alpha_{i s}^{2}}\right\}$.

Assim como Artes (2000) e Venezuela (2008), com o objetivo de diminuir o número de parâmetros de perturbação, assumimos que as equações de estimação são independentes, isto é, $\operatorname{Cov}\left(\mathbf{u}_{i}, \mathbf{v}_{i}\right)=\mathbf{0}$; e que não há dependência entre os elementos de $\mathbf{v}_{i}$, ou seja, $\operatorname{Corr}\left(\mathbf{v}_{i}\right)=\mathbf{I}_{\mathbf{s}}$.

A função de estimação proposta para $\boldsymbol{\theta}$ é dada então por,

$$
\begin{aligned}
\boldsymbol{\Gamma}_{n}(\boldsymbol{\theta}) & =\left(\begin{array}{c}
\boldsymbol{\Gamma}_{n}(\boldsymbol{\beta}) \\
\boldsymbol{\Gamma}_{n}(\boldsymbol{\alpha})
\end{array}\right)=\sum_{i=1}^{n}\left(\begin{array}{c}
\mathbf{X}_{i}^{\top} \mathbf{N}_{i}\left(\boldsymbol{\Sigma}_{i}^{1 / 2} \mathbf{R}_{i}(\rho) \boldsymbol{\Sigma}_{i}^{1 / 2}\right)^{-1} \mathbf{u}_{i} \\
\mathbf{Z}_{i}^{\top} \mathbf{G}_{i} \mathbf{M}_{i} \boldsymbol{\Omega}_{i}^{-1} \mathbf{v}_{i}
\end{array}\right) \\
& =\sum_{i=1}^{n} \mathbf{Q}_{i}^{\top} \boldsymbol{\Lambda}_{i} \mathbf{\Upsilon}_{i}^{-1} \mathbf{d}_{i}=\sum_{i=1}^{n} \mathbf{Q}_{i}^{\top} \mathbf{W}_{i} \boldsymbol{\Lambda}_{i}^{-1} \mathbf{d}_{i},
\end{aligned}
$$

sendo $\mathbf{Q}_{i}=\left(\begin{array}{cc}\mathbf{X}_{i} & \mathbf{0} \\ \mathbf{0} & \mathbf{Z}_{i}\end{array}\right), \boldsymbol{\Lambda}_{i}=\left(\begin{array}{cc}\mathbf{N}_{i} & \mathbf{0} \\ \mathbf{0} & \mathbf{G}_{i} \mathbf{M}_{i}\end{array}\right), \mathbf{W}_{i}=\boldsymbol{\Lambda}_{i} \mathbf{\Upsilon}_{i}^{-1} \boldsymbol{\Lambda}_{i}$ e $\mathbf{\Upsilon}_{i}=\left(\begin{array}{cc}\boldsymbol{\Sigma}_{i}^{1 / 2} \mathbf{R}_{i}(\rho) \boldsymbol{\Sigma}_{i}^{1 / 2} & \mathbf{0} \\ \mathbf{0} & \boldsymbol{\Omega}_{i}\end{array}\right)$.

\subsubsection{Algoritmo de estimação}

Para encontrarmos o estimador $\hat{\boldsymbol{\theta}}$ de $\boldsymbol{\theta}$ devemos resolver a seguinte equação de estimação:

$$
\boldsymbol{\Gamma}_{n}(\hat{\boldsymbol{\theta}})=\mathbf{0}
$$

ou, equivalentemente,

$$
\sum_{i=1}^{n} \mathbf{Q}_{i}^{\top} \hat{\mathbf{\Lambda}}_{i} \hat{\mathbf{\Upsilon}}_{i}^{-1} \hat{\mathbf{d}}_{i}=\mathbf{0}
$$

Aplicando-se o método de Newton-Raphson modificado, assim como na Seção 2.2.2, chegou-se ao seguinte processo iterativo:

$$
\boldsymbol{\theta}^{(m+1)}=\boldsymbol{\theta}^{(m)}-\left\{\mathrm{E}\left[\frac{\partial}{\partial \boldsymbol{\theta}^{\top}} \boldsymbol{\Gamma}\left(\boldsymbol{\theta}^{(m)}\right)\right]\right\}^{-1} \boldsymbol{\Gamma}\left(\boldsymbol{\theta}^{(m)}\right)
$$

em que,

$$
\begin{aligned}
\mathrm{E}\left[\frac{\partial}{\partial \boldsymbol{\theta}^{\top}} \boldsymbol{\Gamma}\left(\boldsymbol{\theta}^{(m)}\right)\right] & =\left(\begin{array}{cc}
\sum_{i=1}^{n} \mathbf{X}_{i} \hat{\mathbf{N}}_{i} \hat{\boldsymbol{\Sigma}}_{i}^{-1 / 2} \mathbf{R}_{i}(\hat{\rho}) \hat{\boldsymbol{\Sigma}}_{i}^{-1 / 2} \hat{\mathbf{N}}_{i} \mathbf{X}_{i} & \mathbf{0} \\
\mathbf{0} & \sum_{i=1}^{n} \mathbf{Z}_{i}^{\top} \mathbf{H}_{i} \mathbf{M}_{i} \boldsymbol{\Omega}_{i}^{-1} \mathbf{M}_{i} \mathbf{H}_{i} \mathbf{Z}_{i}
\end{array}\right) \\
& =\sum_{i=1}^{n} \mathbf{Q}_{i}^{\top} \boldsymbol{\Lambda}_{i} \mathbf{\Upsilon}_{i}^{-1} \boldsymbol{\Lambda}_{i} \mathbf{Q}_{i}^{\top}
\end{aligned}
$$

Portanto,

$$
\boldsymbol{\theta}^{(m+1)}=\boldsymbol{\theta}^{(m)}-\left\{\left[\sum_{i=1}^{n} \mathbf{Q}_{i}^{\top} \boldsymbol{\Lambda}_{i} \mathbf{\Upsilon}_{i}^{-1} \boldsymbol{\Lambda}_{i} \mathbf{Q}_{i}\right]^{-1}\left[\sum_{i=1}^{n} \mathbf{Q}_{i}^{\top} \boldsymbol{\Lambda}_{i} \mathbf{\Upsilon}_{i}^{-1} \mathbf{d}_{i}\right]\right\}^{(m)}
$$




$$
=\boldsymbol{\theta}^{(m)}-\left\{\left[\sum_{i=1}^{n} \mathbf{Q}_{i}^{\top} \mathbf{W}_{i} \mathbf{Q}_{i}\right]^{-1}\left[\sum_{i=1}^{n} \mathbf{Q}_{i}^{\top} \mathbf{W}_{i} \mathbf{\Lambda}_{i}^{-1} \mathbf{d}_{i}\right]\right\}^{(m)},
$$

sendo $\mathbf{W}_{i}=\boldsymbol{\Lambda}_{i} \boldsymbol{\Upsilon}_{i}^{-1} \boldsymbol{\Lambda}_{i}$ e $m$ o número de iterações. Os cálculos para obter a matriz (4.5) estão no Apêndice F. O índice $m$ no lado direito das equações acima indica que as matrizes e os vetores são atualizados pelas estimativas de $\boldsymbol{\beta}, \boldsymbol{\alpha}$, e $\rho$ da $m$-ésima iteração. Para estimar $\rho$, procedemos como descrito na Seção 2.2.2.

A equação (4.6) reescrita na forma do processo iterativo de mínimos quadrados reponderados fica expressa por

$$
\boldsymbol{\theta}^{(m+1)}=\left\{\left[\sum_{i=1}^{n} \mathbf{Q}_{i}^{\top} \mathbf{W}_{i} \mathbf{Q}_{i}\right]^{-1}\left[\sum_{i=1}^{n} \mathbf{Q}_{i}^{\top} \mathbf{W}_{i} \mathbf{z}_{i}\right]\right\}^{(m)},
$$

que emprega uma matriz de pesos $\mathbf{W}_{i}$ e uma variável dependente modificada $\mathbf{z}_{i}$, sendo $\mathbf{z}_{i}=\boldsymbol{\nu}_{i}-$ $\boldsymbol{\Lambda}_{i}^{-1} \mathbf{d}_{i}$.

\section{Processo iterativo}

\section{Processo iterativo}

1) Para determinar o chute inicial do processo iterativo, $\boldsymbol{\theta}^{(0)}$, supomos independência entre as observações da mesma unidade experimental. Ajustamos modelos de regressão linear de $\mathbf{y}$ sobre $\mathbf{X}$ e $\mathbf{Z}$ para obter, respectivamente, valores iniciais: $\boldsymbol{\beta}^{(0)}$ e $\boldsymbol{\gamma}^{(0)}$ (via mínimos quadrados); 2) selecionamos a estrutura de correlação que será utilizada no modelo, consideramos os valores iniciais e estimamos $\rho$ a partir desta estrutura;

3) atualizamos $\boldsymbol{\theta}^{(m)}$ pelo processo iterativo dado por (4.7).

4) repetimos os passos 2 e 3 até a convergência de $\boldsymbol{\theta}$.

\subsubsection{Inferência}

O Teorema 4.1 descreve condições para que o estimador de $\boldsymbol{\theta}$ obtido por (4.7) seja consistente e assintoticamente normal.

Teorema 4.1 Seja $\hat{\boldsymbol{\theta}}$ a raiz de (4.7). Sob condições gerais de regularidade e assumindo que $\hat{\rho}(\boldsymbol{\theta})$ é um estimador $\sqrt{n}$-consistente de $\rho$ dado $\boldsymbol{\theta}$, então temos que $\hat{\boldsymbol{\theta}}$ é um estimador consistente de $\boldsymbol{\theta}$ e

$$
\sqrt{n}\left(\hat{\boldsymbol{\theta}}_{n}-\boldsymbol{\theta}\right) \rightarrow^{D} N_{p+q}\left(\mathbf{0}, \mathbf{J}^{-1}\right)
$$

$\operatorname{com} \mathbf{J}=\lim _{n \longrightarrow} \longrightarrow \mathbf{J}_{n} / n$, sendo $\mathbf{J}_{n}$ a matriz de informação de Godambe de $\boldsymbol{\theta}$ associada à $\boldsymbol{\Gamma}$ dada por

$$
\mathbf{J}_{n}=\left\{\sum_{i=1}^{n} \mathbf{S}_{i}(\boldsymbol{\theta})\right\}\left\{\sum_{i=1}^{n} \mathbf{V}_{i}(\theta)\right\}^{-1}\left\{\sum_{i=1}^{n} \mathbf{S}_{i}(\boldsymbol{\theta})\right\}
$$

A demonstração segue análoga à encontrada em Artes (1997, Teorema 7, p. 67). 
No caso em que os parâmetros $\boldsymbol{\mu}$ e $\boldsymbol{\alpha}$ são ajustados conjuntamente segundo a equação (4.7), o estimador robusto para a matriz de variância-covariâncias de $\boldsymbol{\theta}$ é dado por

$$
\hat{\mathbf{J}}_{n}^{-1}(\boldsymbol{\theta})=\left\{\sum_{i=1}^{n} \hat{\mathbf{S}}_{i}(\boldsymbol{\theta})\right\}^{-1}\left\{\sum_{i=1}^{n} \mathbf{Q}_{i}^{\top} \hat{\mathbf{\Lambda}}_{i} \hat{\mathbf{\Upsilon}}_{i}^{-1} \hat{\mathbf{d}}_{i} \hat{\mathbf{d}}_{i}^{\top} \hat{\mathbf{\Upsilon}}_{i}^{-1} \hat{\boldsymbol{\Lambda}}_{i}^{\top} \mathbf{Q}_{i}\right\}\left\{\sum_{i=1}^{n} \mathbf{S}_{i}(\boldsymbol{\theta})\right\}^{-1},
$$

em que $\mathbf{S}_{i}(\boldsymbol{\theta})=\sum_{i=1}^{n} \mathbf{Q}_{i}^{\top} \boldsymbol{\Lambda}_{i} \mathbf{\Upsilon}_{i}^{-1} \boldsymbol{\Lambda}_{i} \mathbf{Q}_{i}$. As estimativas dessas duas expressões são obtidas substituindo $\boldsymbol{\theta}$ e $\rho$ por suas respectivas estimativas consistentes.

Testes de hipóteses para testar $H_{0}: \boldsymbol{\theta}_{1}=0$ contra $H_{1}: \boldsymbol{\theta}_{1} \neq 0$, em que $\boldsymbol{\theta}=\left(\begin{array}{ll}\boldsymbol{\theta}_{1}^{\top} & \boldsymbol{\theta}_{2}^{\top}\end{array}\right)^{\top}$ podem ser desenvolvidos através de estatísticas tipo Wald, $\xi_{W}=\hat{\boldsymbol{\theta}}_{1}^{\top} \hat{\operatorname{Var}}^{-1}\left(\hat{\boldsymbol{\theta}}_{1}\right) \hat{\boldsymbol{\theta}}_{1}$ com a matriz de variância-covariância estimada por $\mathbf{J}_{n}^{-1}(\hat{\boldsymbol{\theta}})$.

\subsubsection{Estudo de simulação}

Nesta Seção iremos analisar dados log-BS correlacionados heterogêneos. Assim como na Seção 2.2.4, os componentes de $\boldsymbol{\mu}_{i}$ são modelados por

$$
\mu_{i j}=\beta_{0}+\beta_{1} x_{i j}
$$

em que $x_{i j}$ 's denotam valores fixos gerados de uma distribuição uniforme no intervalo $[0,1]$ e $\beta_{0}=4$, $\beta_{1}=-2$. Para introduzir o parâmetro de forma variável, os componentes de $\boldsymbol{\alpha}_{i}$ são modelados como

$$
\alpha_{i j}=\exp \left(\gamma_{0}+\gamma_{1} \mathbf{z}_{i j}\right)
$$

em que $z_{i j}$ 's denotam valores fixos gerados de distribuições uniformes nos intervalo $[0,1]$ e $[0,1.4]$ e os parâmetros que compõem a parte sistemática do parâmetro de forma também variaram. O grau de heterogeneidade do parâmetro $\alpha$ é medido por $\lambda=\max \left(\alpha_{i j}\right) / \min \left(\alpha_{i j}\right)$.

Assim como na Seção 2.2.4 vamos considerar $\rho=0.3,0.6$ e 0.9, tamanhos amostrais $n=10,20,50$ e 80 e $s=3,5$ e 10 . O viés relativo (VR) de $\theta_{j}$ é estimado como $100 \times\left|\bar{\theta}_{j}-\theta_{j}\right| / \theta_{j}$, com $\bar{\theta}_{j}=$ $R^{-1} \sum_{r=1}^{R} \hat{\theta}_{j}^{(r)}, \operatorname{com} \theta_{j}=\left(\beta_{0}, \beta_{1}, \gamma_{0}, \gamma_{1}\right), \hat{\theta}_{j}^{(r)}$ sendo a estimativa EEG de $\theta_{j}$ na $r$-ésima réplica, para $j=0,1, \ldots, R$, e o erro quadrático médio (EQM) é dado por $R^{-1} \sum_{r=1}^{R}\left(\theta_{j}-\hat{\beta}_{j}^{(r)}\right)^{2}$. Consideramos para cada caso $R=5000$ réplicas.

Iremos trabalhar com 2 cenários diferentes: no cenário $1 z_{i j}$ 's denotam valores fixos gerados de uma distribuição uniforme nos intervalo $[0,1]$ e $\gamma_{0}=-2$ e $\gamma_{1}=2$, no cenário $2 z_{i j}$ 's denotam valores fixos gerados de uma distribuição uniforme nos intervalo $[0,(1,4)]$ e $\gamma_{0}=-1$ e $\gamma_{1}=-1$. Para cada cenário consideramos 3 casos: a) os dados são gerados e ajustados levando em consideração uma estrutura de correlação autoregressiva de primeira ordem, b) os dados são gerados e ajustados levando em consideração uma estrutura de correlação permutável, c) os dados são gerados considerando estrutura de correlação autoregressiva e ajustados usando estrutura de correlação permutável. Ou seja, nos casos (a) e (b) escolhemos a estrutura de correlação correta e no caso (c) consideramos uma especificação incorreta da estrutura de correlação. No cenário 1 lidamos com um grau de heterogeneidade baixo, $\lambda \approx 7$, já no cenário 2 temos $\lambda \approx 63$.

As Tabelas G.1-G.6 no Apêndice G apresentam os resultados do estudo de simulação. Percebemos que o comportamento das estimativas de $\beta_{0}$ e $\beta_{1}$ é semelhante ao estudo de simulação realizado 
na Seção 2.2.4, com resultados consistentes e que melhoram conforme aumenta o tamanho amostral e o tamanho do grupo.

Podemos observar também que os erros quadráticos médios das estimativas de $\gamma_{0}$ e $\gamma_{1}$ diminuem quando as amostras crescem e as demais características são fixas (correlação e tamanho grupo), o mesmo ocorre quando fixamos o tamanho amostral e a correlação e comparamos e o grupo aumenta, ou quando fixamos o tamanho do grupo e o tamanho amostral e quem cresce é a correlação. Podemos ainda observar que para o cenário 2, que possui maior grau de heterogeneidade, os EQM's são menores do que no cenário 1.

\subsubsection{Métodos de diagnóstico}

Durante a Seção 2.2.5 foram discutidos métodos de diagnósticos para modelos log-BS homogêneos obtidos a partir da abordagem EEGs. Nesta seção iremos estender e apresentar as medidas estudadas anteriormente para o caso do modelo log-BS heterogêneo.

\section{Análise de resíduos}

No caso dos modelos heterogêneos log-BS discutidos anteriormente, a estimativa $\hat{\boldsymbol{\theta}}$ na convergência do processo iterativo fica expressa na forma,

$$
\hat{\boldsymbol{\theta}}=\left(\mathbf{Q}^{\top} \widehat{\mathbf{W}} \mathbf{Q}\right)^{-1} \mathbf{Q}^{\top} \widehat{\mathbf{W}} \widehat{\mathbf{z}}
$$

em que $\mathbf{Q}=\left[\mathbf{Q}_{1}^{\top}, \ldots, \mathbf{Q}_{n}^{\top}\right]^{\top}$ é $\left(\sum s_{i} \times p\right)$ matriz modelo, $\widehat{\mathbf{W}}=\operatorname{diag}\left\{\widehat{\mathbf{W}}_{1}, \ldots, \widehat{\mathbf{W}}_{n}\right\}$ é $\left(\sum s_{i} \times \sum s_{i}\right)$ matriz de pesos, enquanto $\mathbf{z}=\left(\mathbf{z}_{1}^{\top}, \ldots, \mathbf{z}_{n}^{\top}\right)^{\top} \operatorname{com} \mathbf{z}_{i}=\mathbf{Q}_{i} \boldsymbol{\theta}-\Lambda_{i}^{-1} \mathbf{d}_{i}$ sendo uma pseudo-resposta para o $i$-ésimo grupo, para $i=1, \ldots, n$. De forma que $\hat{\boldsymbol{\theta}}$ é interpretada como a solução de mínimos quadrados da regressão linear de $\widehat{\mathbf{W}}^{1 / 2} \mathbf{z}$ tendo como matriz de planejamento $\widehat{\mathbf{W}}^{1 / 2} \mathbf{Q}$.

A matriz de projeção ortogonal de $\widehat{\mathbf{z}}$ sobre o subespaço vetorial gerado pelas colunas de $\widehat{\mathbf{W}}^{1 / 2} \mathbf{Q}$ é dada por

$$
\widehat{\mathbf{H}}=\operatorname{diag}\left\{\widehat{\mathbf{H}}_{1}, \ldots, \widehat{\mathbf{H}}_{n}\right\},
$$

$\operatorname{com} \mathbf{H}_{i}=\widehat{\mathbf{W}}_{i}^{\frac{1}{2}} \mathbf{Q}_{i}\left(\mathbf{Q}^{\top} \widehat{\mathbf{W}} \mathbf{Q}\right)^{-1} \mathbf{Q}_{i}^{\top} \widehat{\mathbf{W}}_{i}^{\frac{1}{2}}, \mathbf{H}_{i}$ sendo uma matriz $\left(\sum s_{i} \times \sum s_{i}\right)$, para $i=1, \ldots, n$.

Os resíduos ordinários para avaliar a solução de mínimos quadrados podem ser definidos como

$$
\begin{aligned}
r_{i j} & =\widehat{\mathbf{W}}_{i}^{1 / 2} \mathbf{z}_{i}-\widehat{\mathbf{W}}_{i}^{1 / 2} \mathbf{Q}_{i} \widehat{\boldsymbol{\theta}}_{i} \\
& =\widehat{\mathbf{W}}_{i}^{\frac{1}{2}}\left(\widehat{z}_{i}-\mathbf{Q}_{i} \hat{\boldsymbol{\theta}}\right)
\end{aligned}
$$

Portanto,

$$
r_{i j}=\mathbf{e}_{i}^{\top} \widehat{\mathbf{W}}_{i}^{\frac{1}{2}}\left(\widehat{\mathbf{z}}_{i}-\mathbf{Q}_{i} \hat{\boldsymbol{\theta}}\right)
$$

em que $\mathbf{e}_{i}$ é um vetor $\left(\sum s_{i} \times 1\right)$ de zeros com 1 na $j$-ésima posição. Também podemos escrever $r_{i j}=\widehat{\mathbf{W}}_{i}^{1 / 2} \mathbf{z}_{i}-\mathbf{H}_{i} \widehat{\mathbf{W}}_{i}^{\frac{1}{2}} \mathbf{z}_{i}$. Similarmente, definimos uma versão do resíduo studentizado como

$$
t_{r_{i j}}=\frac{\mathbf{e}_{i}^{\top} \widehat{\mathbf{W}}_{i}^{\frac{1}{2}}\left(\widehat{z}_{i}-\mathbf{Q}_{i} \hat{\boldsymbol{\theta}}\right)}{\sqrt{1-\widehat{h}_{i j j}}}
$$


para $i=1, \ldots, n$ e $j=1, \ldots, s_{i}$.

\section{Distância de Cook}

Obtemos para o caso do modelo log-BS heterogêneo a seguinte aproximação de um passo:

$$
\boldsymbol{\theta}_{(i j)}^{1}=\hat{\boldsymbol{\theta}}-\frac{r_{i j}}{\left(1-h_{i j j}\right)} \sqrt{\hat{\omega}_{i j}}\left(\mathbf{Q}^{\top} \widehat{\mathbf{W}} \mathbf{Q}\right)^{-1} \mathbf{q}_{i j}
$$

em que $\boldsymbol{\theta}_{(i j)}^{1}$ denota a aproximação para a estimativa $\hat{\boldsymbol{\theta}}_{(i j)}$, após remover a $j$-ésima medida da $i$-ésima unidade experimental. Então, a distância de Cook aproximada fica dada por

$$
\begin{aligned}
\mathrm{C}_{i j} & =\frac{1}{p}\left(\boldsymbol{\theta}_{(i j)}^{1}-\hat{\boldsymbol{\theta}}\right)^{\top}\left(\mathbf{Q}^{\top} \widehat{\mathbf{W}} \mathbf{Q}\right)^{-1}\left(\boldsymbol{\theta}_{(i j)}^{1}-\hat{\boldsymbol{\theta}}\right) \\
& =\frac{1}{p} r_{i j}^{2} \frac{\hat{h}_{i j j}}{\left(1-\hat{h}_{i j j}\right)} .
\end{aligned}
$$

\section{Influência conformal}

As matrizes para o estudo de influêncial local são obtidas de forma similar ao que foi descrito na Seção 2.2.5. Apresentaremos aqui apenas a matriz $\Delta$ para cada esquema de perturbação em estudo.

i) Ponderação de casos

Aqui o esquema de perturbação é dado por

$$
\boldsymbol{\Gamma}(\boldsymbol{\theta} \mid \boldsymbol{\omega})=\mathbf{Q}^{\top} \mathbf{W} \boldsymbol{\Lambda}^{-1} \operatorname{diag}(\boldsymbol{\omega}) \mathbf{d},
$$

em que $\boldsymbol{\omega}=\left(\boldsymbol{\omega}_{1}^{\top}, \ldots, \boldsymbol{\omega}_{n}^{\top}\right)^{\top} \operatorname{com} \boldsymbol{\omega}_{i}=\left(\omega_{i 1}, \ldots, \omega_{i s}\right)^{\top}, i=1, \ldots, n$ e o vetor que representa a ausência de perturbação, $\boldsymbol{\omega}_{0}$, assume $\omega_{i j}=1$, para toda $i=1, \ldots, n$ and $j=1, \ldots, s$.

A matriz $\boldsymbol{\Delta}$ é a derivada de $\boldsymbol{\Gamma}(\boldsymbol{\theta} \mid \boldsymbol{\omega})$ em relação a $\boldsymbol{\omega}$ resultando em

$$
\boldsymbol{\Delta}=\mathbf{Q}^{\top} \mathbf{W} \mathbf{\Lambda}^{-1} \mathbf{D}
$$

$\operatorname{com} \mathbf{D}=\{\operatorname{diag}(\mathbf{u}), \operatorname{diag}(\mathbf{v})\}^{\top}$ a qual deve ser avaliada em $\boldsymbol{\theta}=\hat{\boldsymbol{\theta}}$ e $\boldsymbol{\omega}=\boldsymbol{\omega}_{0}$.

ii) Perturbação da variável resposta

Considerando a perturbação da variável resposta dada em (2.15) temos que

$$
\boldsymbol{\Gamma}(\boldsymbol{\theta} \mid \boldsymbol{\omega})=\mathbf{Q}^{\top} \mathbf{W} \boldsymbol{\Lambda}^{-1} \mathbf{d}_{\omega}
$$

em que $\mathbf{d}_{\omega}=\left(\mathbf{d}_{\omega 1}^{\top}, \ldots, \mathbf{d}_{\omega n}^{\top}\right)^{\top}, \operatorname{com} \mathbf{d}_{\omega i}=\left(d_{\omega i 1}^{\top}, \ldots, d_{\omega i s}^{\top}\right)^{\top}$

$$
\boldsymbol{\Delta}=\frac{\partial \boldsymbol{\Gamma}(\boldsymbol{\theta} \mid \boldsymbol{\omega})}{\partial \boldsymbol{\omega}^{\top}}=\mathbf{Q}^{\top} \mathbf{W} \boldsymbol{\Lambda}^{-1} \frac{\partial \mathbf{d}_{\omega}}{\partial \boldsymbol{\omega}^{\top}}
$$

avaliado em $\hat{\boldsymbol{\theta}}$ e em $\boldsymbol{\omega}_{0}$. O vetor perturbado na variável resposta para a $i$-ésima unidade experimental fica dado por $\mathbf{d}_{\omega i}=\left(\mathbf{u}_{\omega i}^{\top}, \mathbf{v}_{\omega_{i}}^{\top}\right)^{\top}$, em que $\mathbf{u}_{\omega i}=\left(u_{\omega i 1}, \ldots, u_{\omega i s}\right)^{\top}$ e $\mathbf{v}_{\omega i}=\left(v_{\omega i 1}, \ldots, v_{\omega i s}\right)^{\top}$, com $u_{\omega i j}$ definido em (2.16) com derivada em relação a $\omega_{i j}$ dada em (2.17). 
Por outro lado,

$$
v_{\omega i j}=\frac{1}{\alpha_{i j}}\left[\frac{4}{\alpha_{i j}^{2}} \operatorname{senh}^{2}\left(\frac{y_{\omega i j}-\mu_{i j}}{2}\right)-1\right]
$$

cuja a derivada em relação a $\omega_{i j}$ é dada por,

$$
\frac{\partial v_{\omega_{i j}}}{\partial \omega_{i j}}=s_{y_{i j}} \frac{2}{\alpha^{3}} \sinh \left(y_{\omega i j}-\mu_{i j}\right) .
$$

Portanto,

$$
\frac{\partial \mathbf{d}}{\partial \boldsymbol{\omega}^{\top}}=\left(\begin{array}{c}
\dot{\mathcal{U}} \\
\dot{\mathcal{V}}
\end{array}\right)
$$

$\operatorname{com} \dot{\mathcal{U}}=\operatorname{diag}\left\{\dot{\mathcal{U}}_{1}, \ldots, \dot{\mathcal{U}}_{n}\right\}$ e $\dot{\mathcal{V}}=\operatorname{diag}\left\{\dot{\mathcal{V}}_{1}, \ldots, \dot{\mathcal{V}}_{n}\right\}$ em que $\dot{\mathcal{U}}_{i}=\operatorname{diag}\left\{\frac{\partial u_{\omega i 1}}{\partial \omega_{i 1}}, \ldots, \frac{\partial u_{\omega i s}}{\partial \omega_{i s}}\right\}$ e $\dot{\mathcal{V}}_{i}=$ $\operatorname{diag}\left\{\frac{\partial v_{\omega i 1}}{\partial \omega_{i 1}}, \ldots, \frac{\partial v_{\omega i s}}{\partial \omega_{i s}}\right\}$.

iii) Perturbação individual das covariáveis

Conforme a proposta de Thomas e Cook (1990) o esquema de perturbação considerado é na k-ésima coluna da matriz de covariáveis X. Assim, o vetor perturbado tem cada componente igual ao definido em (2.18).

Assim, perturbando a equação de estimação correspondente temos que,

$$
\boldsymbol{\Gamma}(\boldsymbol{\theta} \mid \boldsymbol{\omega})=\mathbf{Q}_{\omega}^{\top} \boldsymbol{\Lambda}_{\omega} \mathbf{\Upsilon}_{\omega}^{-1} \mathbf{d}_{\omega}
$$

De modo que,

$$
\frac{\partial \boldsymbol{\Gamma}(\boldsymbol{\theta} \mid \boldsymbol{\omega})}{\partial \omega_{i j}}=\mathbf{Q}_{\omega}^{\top} \boldsymbol{\Lambda}_{\omega}\left[\frac{\partial \mathbf{\Upsilon}_{\omega}^{-1}}{\partial \omega_{i j}} \mathbf{d}_{\omega}+\mathbf{\Upsilon}_{\omega} \frac{\partial \mathbf{d}_{\omega}}{\partial \omega_{i j}}\right]+\left[\frac{\partial \mathbf{Q}_{\omega}^{\top}}{\partial \omega_{i j}} \boldsymbol{\Lambda}_{\omega}+\mathbf{Q}_{\omega}^{\top} \frac{\partial \boldsymbol{\Lambda}_{\omega}}{\partial \omega_{i j}}\right] \mathbf{\Upsilon}_{\omega}^{-1} \mathbf{d}_{\omega}
$$

avaliada em $\hat{\boldsymbol{\theta}}$ e $\boldsymbol{\omega}_{0}$, em que

$$
\frac{\partial \mathbf{\Upsilon}_{\omega}^{-1}}{\partial \omega_{i j}}=-\mathbf{\Upsilon}_{\omega}^{-1} \frac{\partial \mathbf{\Upsilon}_{\omega}}{\partial \omega_{i j}} \mathbf{\Upsilon}_{\omega}^{-1}
$$

Quando os parâmetros de localização e forma são modelados conjuntamente, consideremos os seguintes cenários que relacionam as matrizes que modelam a média e a forma (matrizes X e Z, respetivamente): as matrizes $\mathrm{X}$ e $\mathrm{Z}$ são iguais, as matrizes $\mathrm{X}$ e $\mathrm{Z}$ são totalmente diferentes e as matrizes X e Z são parcialmente diferentes.

a) Matriz $Z$ igual Matriz $X$

Neste caso a matriz de covariáveis é dada por, $\mathbf{Q}^{\top}=\left(\begin{array}{cc}\mathbf{X}_{\omega}^{\top} & \mathbf{0} \\ \mathbf{0} & \mathbf{X}_{\omega}^{\top}\end{array}\right)$, de modo que, $\frac{\partial \mathbf{Q}^{\top}}{\partial \boldsymbol{\omega}^{\top}}=$ $\left(\begin{array}{cc}\frac{\partial \mathbf{X}_{\omega}^{\top}}{\partial \boldsymbol{\omega}^{\top}} & \mathbf{0} \\ \mathbf{0} & \frac{\partial \mathbf{X}_{\omega}^{\top}}{\partial \boldsymbol{\omega}^{\top}}\end{array}\right)$ é uma matriz $(2 p) \times 2 N,(N=n \times s)$ em que a derivada de $\mathbf{X}_{\omega}^{\top}$ com relação a $\omega^{\top}$ é uma matriz $p \times N$ de zeros exceto a k-ésima linha que é composta pela constante $s_{x k}$. As 
derivadas de $\Upsilon_{\omega}, \mathbf{d}_{\omega}$ e $\boldsymbol{\Lambda}_{\omega}$ em relação $\boldsymbol{\omega}^{\top}$ são apresentadas a seguir. Temos que,

$$
\boldsymbol{\Lambda}_{\omega i}=\left(\begin{array}{cc}
\mathbf{N}_{\omega i} & \mathbf{0} \\
\mathbf{0} & \mathbf{G}_{\omega i} \mathbf{M}_{\omega i}
\end{array}\right), \quad \Upsilon_{\omega i}=\left(\begin{array}{cc}
\boldsymbol{\Sigma}_{\omega i}^{1 / 2} \mathbf{R}(\rho) \boldsymbol{\Sigma}_{\omega i}^{1 / 2} & \mathbf{0} \\
\mathbf{0} & \boldsymbol{\Omega}_{\omega i}
\end{array}\right) \text { e } \mathbf{d}_{\omega i}=\left(\begin{array}{c}
\mathbf{u}_{\omega i} \\
\mathbf{v}_{\omega i}
\end{array}\right)
$$

em que $\mathbf{N}_{\omega i}=\operatorname{diag}\left\{\mathrm{E}\left(\dot{u}_{\omega i 1}\right), \ldots, \mathrm{E}\left(\dot{u}_{\omega i s}\right)\right\}, \operatorname{com} \mathrm{E}\left(\dot{u}_{\omega i j}\right)=\frac{1}{4} \mathrm{E}\left\{\operatorname{sech}^{2}\left(\frac{y_{i j}-\mu_{\omega i j}}{2}\right)\right\}-\frac{1}{2}-\frac{1}{\alpha_{\omega i j}^{2}}$, $\boldsymbol{\Sigma}_{\omega i}=\operatorname{diag}\left\{\operatorname{Var}\left(u_{\omega i 1}\right), \ldots, \operatorname{Var}\left(u_{\omega i s}\right)\right\}, \operatorname{com} \operatorname{Var}\left(u_{\omega i j}\right)=\mathrm{E}\left\{\frac{1}{4} \operatorname{tgh}^{2}\left(\frac{y_{i j}-\mu_{\omega i j}}{2}\right)\right\}+\frac{1}{4}+\frac{1}{\alpha_{\omega i j}^{2}}, \mathbf{G}_{\omega i}=$ $\operatorname{diag}\left\{\partial g^{-1}\left(\delta_{\omega i 1}\right) / \partial \delta_{i 1}, \ldots, \partial g^{-1}\left(\delta_{\omega i s}\right) / \partial \delta_{i s}\right\}, \mathbf{M}_{\omega i}=\operatorname{diag}\left\{-\frac{2}{\alpha_{\omega i j}^{2}}, \ldots,-\frac{2}{\alpha_{\omega i s}^{2}}\right\}$

e $\boldsymbol{\Omega}_{\omega i}=\operatorname{diag}\left\{\frac{2}{\alpha_{\omega i 1}^{2}}, \ldots, \frac{2}{\alpha_{\omega i s}^{2}}\right\}$. Assim, obtemos que,

$$
\begin{aligned}
\frac{\partial \boldsymbol{\Lambda}_{\omega i}}{\partial \boldsymbol{\omega}_{i}^{\top}} & =\left(\begin{array}{cc}
2 \mathbf{G}_{\omega i} \dot{\mathbf{M}}_{\omega i} \gamma_{k} s_{x k} & \mathbf{0} \\
\mathbf{0} & \dot{\mathbf{G}}_{\omega i} \mathbf{M}_{\omega i}+\mathbf{G}_{\omega i} \dot{\mathbf{M}}_{\omega i}
\end{array}\right), \\
\frac{\partial \boldsymbol{\Upsilon}_{\omega i}}{\partial \boldsymbol{\omega}_{i}^{\top}} & =\left(\begin{array}{cc}
-\frac{1}{4} \gamma_{k} s_{x k}\left[\boldsymbol{\Sigma}_{\omega i}^{1 / 2} \mathbf{R}(\rho) \boldsymbol{\Sigma}_{\omega i}^{-1 / 2} \dot{\mathbf{M}}_{\omega i} \mathbf{G}_{\omega i}+\boldsymbol{\Sigma}_{\omega i}^{-1 / 2} \dot{\mathbf{M}}_{\omega i} \mathbf{G}_{\omega i} \mathbf{R}(\rho) \boldsymbol{\Sigma}_{\omega i}^{1 / 2}\right] & \mathbf{0} \\
\mathbf{0} & \gamma_{k} \sigma_{x_{k}} \dot{\boldsymbol{\Omega}}
\end{array}\right), \\
\frac{\partial \mathbf{d}_{\omega i}}{\partial \boldsymbol{\omega}_{i}^{\top}} & =\left(\begin{array}{c}
\beta_{k} s_{x k} \mathcal{A}_{\omega i}-\mathcal{B}_{\omega i} \mathbf{G}_{\omega i} \gamma_{k} s_{x_{k}} \\
\mathcal{C}_{\omega i} \mathbf{G}_{\omega i} \gamma_{k} s_{x_{k}}-2 \mathbf{M}_{\omega i} \mathbf{G}_{\omega i} \gamma_{k} s_{x_{k}}-\mathcal{B}_{\omega i} \beta_{k} s_{x k}
\end{array}\right) .
\end{aligned}
$$

em que $\frac{\partial \mathbf{G}_{\omega}}{\partial \boldsymbol{\omega}^{\top}}=\operatorname{diag}\left\{\dot{\mathbf{G}}_{\omega 1}, \ldots, \dot{\mathbf{G}}_{\omega s}\right\}, \frac{\partial \mathbf{M}_{\omega}}{\partial \boldsymbol{\omega}^{\top}}=\operatorname{diag}\left\{\dot{\mathbf{M}}_{\omega 1}, \ldots, \dot{\mathbf{M}}_{\omega s}\right\}, \frac{\partial \Omega_{\omega}}{\partial \boldsymbol{\omega}^{\top}}=\operatorname{diag}\left\{\dot{\Omega}_{\omega 1}, \ldots, \dot{\Omega}_{\omega s}\right\}$, $\frac{\partial \mathcal{A}_{\omega i}}{\partial \boldsymbol{\omega}^{\top}}=\operatorname{diag}\left\{\mathcal{A}_{\omega i 1}, \ldots, \mathcal{A}_{\omega i s}\right\}, \frac{\partial \mathcal{B}_{\omega i}}{\partial \boldsymbol{\omega}^{\top}}=\operatorname{diag}\left\{\mathcal{B}_{\omega i 1}, \ldots, \mathcal{B}_{\omega i s}\right\}, \operatorname{com} \dot{\mathbf{M}}_{\omega i}=\operatorname{diag}\left\{\frac{4}{\alpha_{\omega i j}^{3}}, \ldots, \frac{4}{\alpha_{\omega i s}^{3}}\right\}$, $\dot{\mathbf{G}}_{\omega i}=\operatorname{diag}\left\{\partial^{2} g^{-1}\left(\delta_{\omega i 1}\right) / \partial \delta_{i 1}^{2}, \ldots, \partial^{2} g^{-1}\left(\delta_{\omega i s}\right) / \partial \delta_{i s}^{2}\right\}$ e $\dot{\boldsymbol{\Omega}}_{\omega i}=\operatorname{diag}\left\{-\frac{4}{\alpha_{\omega i j}^{3}}, \ldots,-\frac{4}{\alpha_{\omega i s}^{3}}\right\}$. E definimos

$$
\begin{aligned}
& \mathcal{A}_{\omega i j}=\frac{1}{4} \operatorname{sech}^{2}\left(\frac{y_{i j}-\mu_{\omega i j}}{2}\right)-\frac{1}{\alpha_{\omega i j}^{2}} \cosh \left(y_{i j}-\mu_{\omega i j}\right), \quad \mathcal{B}_{\omega i j}=\frac{2}{\alpha_{\omega i j}^{3}} \operatorname{senh}\left(y_{i j}-\mu_{\omega i j}\right) \\
& \mathcal{C}_{\omega i j}=-\frac{12}{\alpha_{i j}^{4}} \operatorname{senh}^{2}\left(\frac{y_{i j}-\mu_{\omega i j}}{2}\right) .
\end{aligned}
$$

\section{b) Matriz $Z$ totalmente diferente da Matriz $X$}

Quando a matriz X, que modela o parâmetro de localização, tem a $k$-ésima coluna perturbada, e a matriz de covariáveis $\mathrm{Z}$ é completamente diferente da matriz $\mathrm{X}$, a matriz de covariáveis perturbada fica dada por, $\mathbf{Q}^{\top}=\left(\begin{array}{cc}\mathbf{X}_{\omega}^{\top} & \mathbf{0} \\ \mathbf{0} & \mathbf{Z}\end{array}\right)$. Logo,

$$
\frac{\partial \mathbf{Q}^{\top}}{\partial \boldsymbol{\omega}^{\top}}=\left(\begin{array}{cc}
\frac{\partial \mathbf{X}_{\omega}^{\top}}{\partial \boldsymbol{\omega}^{\top}} & \mathbf{0} \\
\mathbf{0} & \mathbf{0}
\end{array}\right)
$$

sendo uma matriz $(p+q) \times 2 N$, em que a derivada de $\mathbf{X}_{\omega}^{\top}$ com a relação a $\omega^{\top}$ é uma matriz $p \times N$ em que k-ésima linha é composta pela constante $s_{x k}$ e o restante das entradas é zero. Temos que

$$
\boldsymbol{\Lambda}_{\omega i}=\left(\begin{array}{cc}
\mathbf{N}_{\omega i} & \mathbf{0} \\
\mathbf{0} & \mathbf{G M}
\end{array}\right), \quad \Upsilon_{\omega i}=\left(\begin{array}{cc}
\boldsymbol{\Sigma}_{\omega i}^{1 / 2} \mathbf{R}(\rho) \boldsymbol{\Sigma}_{\omega i}^{1 / 2} & \mathbf{0} \\
\mathbf{0} & \boldsymbol{\Omega}_{i}
\end{array}\right) \text { e } \mathbf{d}_{\omega i}=\left(\begin{array}{c}
\mathbf{u}_{\omega i} \\
\mathbf{v}_{\omega i}
\end{array}\right),
$$


em que $\mathbf{N}_{\omega i}=\operatorname{diag}\left\{\mathrm{E}\left(\dot{u}_{\omega i 1}\right), \ldots, \mathrm{E}\left(\dot{u}_{\omega i s}\right)\right\}, \operatorname{com} \mathrm{E}\left(\dot{u}_{\omega i j}\right)=\frac{1}{4} \mathrm{E}\left\{\operatorname{sech}^{2}\left(\frac{y_{i j}-\mu_{\omega i j}}{2}\right)\right\}-\frac{1}{2}-\frac{1}{\alpha_{i j}^{2}}, \boldsymbol{\Sigma}_{\omega i}=$ $\operatorname{diag}\left\{\operatorname{Var}\left(u_{\omega i 1}\right), \ldots, \operatorname{Var}\left(u_{\omega i s}\right)\right\} \quad\left(\boldsymbol{\Sigma}_{\omega i}^{1 / 2}=\operatorname{diag}\left\{\operatorname{Var}^{1 / 2}\left(u_{\omega i 1}\right), \ldots, \operatorname{Var}^{1 / 2}\left(u_{\omega i s}\right)\right\}\right), \operatorname{com} \operatorname{Var}\left(u_{\omega i j}\right)=$ $\mathrm{E}\left\{\frac{1}{4} \operatorname{tgh}^{2}\left(\frac{y_{i j}-\mu_{\omega i j}}{2}\right)\right\}+\frac{1}{4}+\frac{1}{\alpha_{i j}^{2}}, \mathbf{G}_{i}=\operatorname{diag}\left\{\partial g\left(\delta_{i 1}\right) / \partial \delta_{i 1}, \ldots, \partial g\left(\delta_{i 1}\right) / \partial \delta_{i s}\right\}, \mathbf{M}_{i}=\operatorname{diag}\left\{-\frac{2}{\alpha_{i 1}^{2}}, \ldots,-\frac{2}{\alpha_{i s}^{2}}\right\}$, $\boldsymbol{\Omega}_{i}=\operatorname{diag}\left\{\frac{2}{\alpha_{i 1}^{2}}, \ldots, \frac{2}{\alpha_{i s}^{2}}\right\}, u_{\omega i j}=-\frac{1}{2} \operatorname{tgh}\left(\frac{y_{i j}-\mu_{\omega i j}}{2}\right)+\frac{1}{\alpha_{i j}^{2}} \operatorname{senh}\left(y_{i j}-\mu_{\omega i j}\right) \mathrm{e}$ $v_{i j}=\frac{1}{\alpha_{i j}}\left[\frac{4}{\alpha_{i j}^{2}} \operatorname{senh}^{2}\left(\frac{y_{i j}-\mu_{\omega i j}}{2}\right)-1\right]$.

Assim, obtemos que,

$$
\frac{\partial \boldsymbol{\Lambda}_{\omega i}}{\partial \boldsymbol{\omega}_{i}^{\top}}=\frac{\partial \boldsymbol{\Upsilon}_{\omega i}}{\partial \boldsymbol{\omega}_{i}^{\top}}=\left(\begin{array}{cc}
\mathbf{0} & \mathbf{0} \\
\mathbf{0} & \mathbf{0}
\end{array}\right) \text { e } \frac{\partial \mathbf{d}_{\omega i}}{\partial \boldsymbol{\omega}_{i}^{\top}}=\left(\begin{array}{c}
\beta_{k} s_{x k} \mathcal{A}_{\omega i}^{*} \\
-\mathcal{B}_{\omega i}^{*} \beta_{k} s_{x k}
\end{array}\right)
$$

em que definimos

$$
\mathcal{A}_{\omega i j}^{*}=\frac{1}{4} \operatorname{sech}^{2}\left(\frac{y_{i j}-\mu_{\omega i j}}{2}\right)-\frac{1}{\alpha_{\omega i j}^{2}} \cosh \left(y_{i j}-\mu_{\omega i j}\right), \quad \mathcal{B}_{\omega i j}^{*}=\frac{2}{\alpha_{\omega i j}^{3}} \operatorname{senh}\left(y_{i j}-\mu_{\omega i j}\right) .
$$

c) A k-ésima coluna da matriz $\mathbf{X}$ é igual a l-ésima coluna da matriz $\mathbf{Z}$

Vamos agora considerar o caso em que perturbamos a $k$-ésima coluna da matriz que modela o parâmetro de localização, $\mathbf{X}$, que é igual à $l$-ésima coluna da matriz $\mathbf{Z}$. A matriz de covariáveis é então determinada por, $\mathbf{Q}^{\top}=\left(\begin{array}{cc}\mathbf{X}_{\omega}^{\top} & \mathbf{0} \\ \mathbf{0} & \mathbf{Z}_{\omega}^{\top}\end{array}\right)$, de modo que,

$$
\frac{\partial \mathbf{Q}^{\top}}{\partial \boldsymbol{\omega}^{\top}}=\left(\begin{array}{cc}
\frac{\partial \mathbf{X}_{\omega}^{\top}}{\partial \boldsymbol{\omega}^{\top}} & \mathbf{0} \\
\mathbf{0} & \frac{\partial \mathbf{X}_{\omega}^{\top}}{\partial \boldsymbol{\omega}^{\top}}
\end{array}\right)
$$

é uma matriz $(p+q) \times 2 N,(N=n \times s)$ em que a derivada de $\mathbf{X}_{\omega}^{\top}$ (ou $\left.\mathbf{Z}_{\omega}^{\top}\right)$ com relação a $\boldsymbol{\omega}^{\top}$ é a matriz nula exceto pela $k$-ésima linha ( $l$-ésima linha) que é composta pela constante $s_{x k}$. As derivadas de $\mathbf{X}_{\omega}^{\top}$ e $\mathbf{Z}_{\omega}^{\top}$ com relação a $\boldsymbol{\omega}^{\top}$ possuem dimensões $p \times N$ e $q \times N$, respectivamente. De modo análogo ao item $a$ ) obtemos as derivadas de $\Upsilon_{\omega}, \mathbf{d}_{\omega}$ e $\boldsymbol{\Lambda}_{\omega}$ em relação $\boldsymbol{\omega}^{\top}$. Entretanto, observe que

$$
\frac{\partial \alpha_{\omega i j}}{\partial \omega_{i j}}=\frac{\partial g^{-1}\left(\delta_{\omega i j}\right)}{\partial \delta_{i j}} \gamma_{l} s_{x_{k}}
$$

$\operatorname{com} i=1, \ldots, n, j=1, \ldots, s, k=2, \ldots, p$ e $l=2, \ldots, q$. Portanto, basta substituir em (4.1.5) $\gamma_{k}$ por $\gamma_{l}$.

\subsection{Equação de estimação conjunta para o modelo log-BS-t}

\subsubsection{Derivação de função de estimação conjunta}

Consideramos agora uma amostra aleatória de $n$ unidades amostrais, $\mathbf{t}_{1}, \ldots, \mathbf{t}_{n}$, em que $\mathbf{t}_{i}=$ $\left(\mathrm{t}_{i 1}, \ldots, \mathrm{t}_{i s_{i}}\right)^{\top}$ e admitimos que cada componente $\mathrm{t}_{i j} \sim \mathrm{BS}-\mathrm{t}\left(\alpha_{i j}, \beta_{i j}^{*}, \nu\right)$, de forma que cada compo- 
nente $\mathrm{y}_{i j}=\log \left(\mathrm{t}_{i j}\right) \sim \log$-BS- $t\left(\alpha_{i j}, \mu_{i j}, \nu\right)$, isto é, sua distribuição marginal é dada por

$$
f\left(y_{i j}, \mu_{i j}, \alpha_{i j}\right)=\mathrm{c}(\nu)\left\{\frac{1}{\alpha_{i j}} \cosh \left(\frac{y_{i j}-\mu_{i j}}{2}\right)\right\}\left\{1+\frac{1}{\nu}\left[\frac{2}{\alpha_{i j}} \operatorname{senh}\left(\frac{y_{i j}-\mu_{i j}}{2}\right)\right]^{2}\right\}^{-\left(\frac{\nu+1}{2}\right)},
$$

$\alpha_{i j}>0, i=1, \ldots, n, j=1, \ldots, s_{i}$. Ou seja, vamos incorporar a heterogeneidade do parâmetro de forma ao que foi feito no Capítulo 3. Sem perda de generalidade, assumiremos experimentos balanceados $s_{i}=s$.

O parâmetro de localização é expresso na forma

$$
\mu_{i j}=\eta_{i j}=\mathbf{x}_{i j}^{\top} \boldsymbol{\beta}
$$

adicionalmente, o parâmetro de forma agora assume a forma,

$$
g\left(\alpha_{i j}\right)=\delta_{i j}=\mathbf{z}_{i j}^{\top} \gamma
$$

em que $\boldsymbol{\beta}=\left(\beta_{1}, \ldots \beta_{p}\right)^{\top}$ e $\boldsymbol{\gamma}=\left(\gamma_{1}, \ldots \gamma_{q}\right)^{\top}$ são, respectivamente, vetore $p$ e $q$-dimensionais de parâmetros desconhecidos, $\mathbf{x}_{i j}=\left(x_{i j 1} \ldots x_{i j p}\right)^{\top}$ e $\mathbf{z}_{i j}=\left(z_{i j 1} \ldots z_{i j q}\right)^{\top}$ contém valores de variávéis explicativas associadas a $\mu_{i j}$ e $\alpha_{i j}$, respectivamente, e $g(\cdot)$ é uma função de ligação monótona e duas vezes diferenciável. Devido à bimodalidade da função densidade da SN quando $\alpha>2$, que pode causar múltiplos máximos na função de verossimilhança, é razoável impor que $\alpha_{i j} \leq 2$. Como foi feito no caso clássico será assumido que

$$
\begin{gathered}
\alpha_{i j}=\exp \left(\mathbf{z}_{i j}^{\top} \boldsymbol{\gamma}\right) . \\
\frac{\partial \xi_{i j 2}}{\partial \alpha_{i j}}=-\frac{1}{\alpha_{i j}^{2}} \operatorname{senh}\left(\frac{y_{i j}-\mu_{i j}}{2}\right)=-\frac{1}{\alpha_{i j}} \xi_{i j 2} .
\end{gathered}
$$

Então,

$$
\begin{aligned}
\frac{\partial l\left(\mu_{i j} ; y_{i j}\right)}{\partial \alpha_{i j}} & =\frac{1}{\xi_{i j 1}} \xi_{i j 1}\left(\frac{-1}{\alpha_{i j}}\right)-\frac{\left(\frac{\nu+1}{2}\right)\left\{-\frac{8}{\alpha_{i j}} \xi_{i j 2}^{2}\right\}}{\left\{1+\frac{4}{\nu} \xi_{i j 2}^{2}\right\}} \\
& =\frac{-1}{\alpha_{i j}}+\frac{(\nu+1)\left\{\frac{4}{\alpha_{i j}} \xi_{i j 2}^{2}\right\}}{\left\{\nu+4 \xi_{i j 2}^{2}\right\}} \\
& =\frac{1}{\alpha_{i j}}\left(4 w_{i j} \xi_{i j 2}^{2}-1\right),
\end{aligned}
$$

em que $w_{i j}=\frac{\nu+1}{\nu+4 \xi_{i j 2}^{2}}$.

Portanto, a função escore do parâmetro $\alpha_{i j}$ fica dada por,

$$
v_{i j}==\frac{1}{\alpha_{i j}}\left(4 w_{i j} \xi_{i j 2}^{2}-1\right)
$$


e definimos $\mathbf{v}_{i}=\left(v_{i 1}, \ldots, v_{i s}\right)^{\top}$. Análogo ao que foi feito no Apêndice A podemos mostrar que $\mathbf{v}_{i}$ são vetores com média zero.

Observe que,

$$
\frac{d w_{i j}}{\alpha_{i j}}=\frac{8 w_{i j} \xi_{i j 2}^{2}}{\alpha_{i j}\left(\nu+4 \xi_{i j 2}^{2}\right)}
$$

$\operatorname{logo}$

$$
\begin{aligned}
\dot{v}_{i j}=\frac{\partial v_{i j}}{\partial \alpha_{i j}}= & -\frac{1}{\alpha_{i j}^{2}}\left(4 w_{i j} \xi_{i j 2}^{2}-1\right)+\frac{1}{\alpha_{i j}}\left(4\left(\frac{8 w_{i j} \xi_{i j 2}^{2}}{\alpha_{i j}\left(\nu+4 \xi_{i j 2}^{2}\right)}\right) \xi_{i j 2}^{2}\right. \\
& \left.+4 w_{i j} 2 \xi_{i j 2}\left(-\frac{1}{\alpha_{i j}} \xi_{i j 2}\right)\right) \\
= & \frac{1}{\alpha_{i j}^{2}}+\frac{4 w_{i j} \xi_{i j 2}^{2}}{\alpha_{i j}^{2}}\left\{-1+\frac{8 \xi_{i j 2}^{2}}{\nu+4 \xi_{i j 2}^{2}}-2\right\} .
\end{aligned}
$$

Realizando uma mudança de variável e tomando $x_{i j}=\xi_{i j 2} \alpha$, lembrando que $w_{i j}=\frac{\alpha_{i j}^{2}(\nu+1)}{\nu \alpha_{i j}^{2}+4 x_{i j}^{2}}$, obtemos

$$
\begin{gathered}
\mathrm{E}\left(\dot{v}_{i j}\right)=\int_{-\infty}^{\infty} \quad\left\{\frac{2}{\alpha} \mathrm{c}(\nu)\left\{1+\frac{4}{\nu}\left(\frac{x_{i j}}{\alpha}\right)^{2}\right\}^{-\left(\frac{\nu+1}{2}\right)}\left[\frac{1}{\alpha_{i j}^{2}}+\frac{4 w_{i j} x_{i j}^{2}}{\alpha_{i j}^{4}}\left\{-3+\frac{8 x_{i j}^{2}}{\nu \alpha_{i j}^{2}+4 x_{i j}^{2}}\right\}\right]\right\} \mathrm{d} x_{i j} \\
=-\frac{2 \nu}{(3+\nu) \alpha_{i j}^{2}} .
\end{gathered}
$$

Denotamos,

$$
\mathbf{M}_{i}=\mathrm{E}\left(\frac{\partial \mathbf{v}_{i}}{\partial \boldsymbol{\alpha}_{i}}\right)=\operatorname{diag}\left\{\mathrm{E}\left(\dot{v}_{i}\right), \ldots, \mathrm{E}\left(\dot{v}_{i s}\right)\right\}
$$

portanto,

$$
\mathrm{E}\left(\frac{\partial \mathbf{v}_{i}}{\partial \boldsymbol{\gamma}^{\top}}\right)^{\top}=\mathrm{E}\left(\frac{\partial \mathbf{v}_{i}}{\partial \boldsymbol{\alpha}_{i}} \frac{\partial \boldsymbol{\alpha}_{i}}{\partial \boldsymbol{\delta}_{i}} \frac{\partial \boldsymbol{\delta}_{i}}{\partial \boldsymbol{\gamma}^{\top}}\right)^{\top}=\mathbf{Z}_{i}^{\top} \mathbf{G}_{i} \mathbf{M}_{i}
$$

em que $\mathbf{G}_{i}=\operatorname{diag}\left\{\partial g^{-1}\left(\mathbf{z}_{i 1}^{\top} \boldsymbol{\gamma}\right) / \partial \delta_{i 1}, \ldots, \partial g^{-1}\left(\mathbf{z}_{i s}^{\top} \gamma\right) / \partial \delta_{i s}\right\}$

A matriz de variância-covariância de $\mathbf{v}_{i}$ pode ser escrita na forma

$$
\operatorname{Cov}\left(\mathbf{v}_{i}\right)=\operatorname{Var}\left(\mathbf{v}_{i}\right)^{1 / 2} \operatorname{Corr}\left(\mathbf{v}_{i}\right) \operatorname{Var}\left(\mathbf{v}_{i}\right)^{1 / 2}
$$

em que $\mathbf{R}\left(\mathbf{v}_{i}\right)$ representa a matriz de correlação de $\mathbf{v}_{i}$ e $\operatorname{Var}\left(\mathbf{v}_{i}\right)$ é dada por,

$$
\operatorname{Var}\left(\mathbf{v}_{i}\right)=\operatorname{diag}\left\{\operatorname{Var}\left(v_{i 1}\right), \ldots, \operatorname{Var}\left(v_{i s}\right)\right\}=\operatorname{diag}\left\{\mathrm{E}\left(v_{i 1}^{2}\right), \ldots, \mathrm{E}\left(v_{i s}^{2}\right)\right\},
$$

em que,

$$
v_{i j}^{2}=\left[\frac{1}{\alpha_{i j}}\left(4 w_{i j} \xi_{i j 2}^{2}-1\right)\right]^{2}
$$


Realizando uma mudança de variável e tomando $x_{i j}=\xi_{i j 2} \alpha$ temos que

$$
\begin{aligned}
\operatorname{Var}\left(v_{i j}\right)=\mathrm{E}\left(v_{i j}^{2}\right) & =\int_{-\infty}^{\infty}\left\{\frac{2}{\alpha} \mathrm{c}(\nu)\left\{1+\frac{4}{\nu}\left(\frac{x_{i j}}{\alpha}\right)^{2}\right\}^{-\left(\frac{\nu+1}{2}\right)} \frac{1}{\alpha_{i j}^{2}}\left(4 w_{i j} \frac{x_{i j}^{2}}{\alpha_{i j}^{2}}-1\right)^{2}\right\} d x_{i j} \\
& =\frac{2 \nu}{(3+\nu) \alpha_{i j}^{2}}
\end{aligned}
$$

Denotamos, $\boldsymbol{\Omega}_{i}=\operatorname{diag}\left\{\mathrm{E}\left(v_{i 1}^{2}\right), \ldots, \mathrm{E}\left(v_{i s}^{2}\right)\right\} . \operatorname{Logo}$,

$$
\mathbf{W}_{i}^{*}=\operatorname{Cov}\left(\mathbf{v}_{i}\right)=\operatorname{Var}\left(\mathbf{v}_{i}\right)^{1 / 2} \operatorname{Corr}\left(\mathbf{v}_{i}\right) \operatorname{Var}\left(\mathbf{v}_{i}\right)^{1 / 2}=\boldsymbol{\Omega}_{i}^{1 / 2} \operatorname{Corr}\left(\mathbf{v}_{i}\right) \boldsymbol{\Omega}_{i}^{1 / 2},
$$

em que $\operatorname{Corr}\left(\mathbf{v}_{i}\right)$ é a verdadeira matriz de correlação entre as observações $v_{i 1}, \ldots, v_{i s}$. A função de estimação linear ótima para $\gamma$ fica portanto dada por,

$$
\boldsymbol{\zeta}_{n}(\boldsymbol{\gamma})=\sum_{i=1}^{n} \mathbf{Z}_{i}^{\top} \mathbf{H}_{i} \mathbf{M}_{i} \mathbf{W}_{\mathbf{i}}^{*-1} \mathbf{v}_{i}
$$

Seja $\boldsymbol{\theta}=\left(\boldsymbol{\beta}^{\top}, \boldsymbol{\gamma}^{\top}\right)^{\top}$ e consideremos $\mathbf{d}_{i}=\left(\mathbf{u}_{i}^{\top}, \mathbf{v}_{i}^{\top}\right)^{\top}$. Uma função de estimação ótima para $\boldsymbol{\theta}$ gerada por $\mathbf{d}_{i}$ é dada por,

$$
\boldsymbol{\Gamma}_{n}(\boldsymbol{\theta})^{0}=\mathrm{E} \sum_{i=1}^{n}\left(\begin{array}{cc}
\frac{\partial \mathbf{u}_{i}}{\partial \boldsymbol{\beta}^{\top}} & \frac{\partial \mathbf{u}_{i}}{\partial \boldsymbol{\gamma}^{\top}} \\
\frac{\partial \mathbf{v}_{i}}{\partial \boldsymbol{\beta}^{\top}} & \frac{\partial \mathbf{v}_{i}}{\partial \boldsymbol{\gamma}^{\top}}
\end{array}\right)^{\top} \operatorname{Cov}\left(\mathbf{d}_{i}\right)^{-1} \mathbf{d}_{i} .
$$

Vimos anteriormente que,

$$
\mathrm{E}\left(\frac{\partial \mathbf{u}_{i}}{\partial \boldsymbol{\beta}^{\top}}\right)^{\top}=\mathbf{X}_{i}^{\top} \mathbf{N}_{i} \quad \text { e } \quad \mathrm{E}\left(\frac{\partial \mathbf{v}_{i}}{\partial \boldsymbol{\gamma}^{\top}}\right)^{\top}=\mathbf{Z}_{i}^{\top} \mathbf{H}_{\mathbf{i}} \mathbf{M}_{i} .
$$

Note que,

$$
\mathrm{E}\left(\frac{\partial \mathbf{u}_{i}}{\partial \boldsymbol{\gamma}^{\top}}\right)^{\top}=\mathbf{0} \text { e } \mathrm{E}\left(\frac{\partial \mathbf{v}_{i}}{\partial \boldsymbol{\beta}^{\top}}\right)^{\top}=\mathbf{0} .
$$

Portanto,

$$
\boldsymbol{\Gamma}_{n}(\boldsymbol{\theta})^{0}=\sum_{i=1}^{n}\left(\begin{array}{cc}
\mathbf{X}_{i}^{\top} \mathbf{N}_{i} & \mathbf{0} \\
\mathbf{0} & \mathbf{Z}_{i}^{\top} \mathbf{H}_{\mathbf{i}} \mathbf{M}_{i}
\end{array}\right) \mathbf{A}_{i}^{-1} \mathbf{d}_{i},
$$

com

$$
\begin{aligned}
\mathbf{A}_{i}=\operatorname{cov}\left(\mathbf{d}_{i}\right) & =\left(\begin{array}{cc}
\operatorname{Cov}\left(\mathbf{u}_{i}\right) & \operatorname{Cov}\left(\mathbf{u}_{i}, \mathbf{v}_{i}\right) \\
\operatorname{Cov}\left(\mathbf{u}_{i}, \mathbf{v}_{i}\right) & \operatorname{Cov}\left(\mathbf{v}_{i}\right)
\end{array}\right) \\
& =\left(\begin{array}{cc}
\boldsymbol{\Sigma}_{i}^{1 / 2} \operatorname{Corr}\left(\mathbf{u}_{i}\right) \boldsymbol{\Sigma}_{i}^{1 / 2}, & \operatorname{Cov}\left(\mathbf{u}_{i}, \mathbf{v}_{i}\right) \\
\operatorname{Cov}\left(\mathbf{u}_{i}, \mathbf{v}_{i}\right) & \boldsymbol{\Omega}_{i}^{1 / 2} \operatorname{Corr}\left(\mathbf{v}_{i}\right) \boldsymbol{\Omega}_{i}^{1 / 2}
\end{array}\right),
\end{aligned}
$$

em que $\mathbf{N}_{i}=\mathrm{E}\left(\frac{\partial \mathbf{u}_{i}}{\partial \boldsymbol{\mu}_{i}}\right)=\operatorname{diag}\left\{\mathrm{E}\left(\dot{u}_{i 1}\right), \ldots, \mathrm{E}\left(\dot{u}_{i s}\right)\right\}, \operatorname{com} \mathrm{E}\left(\dot{u}_{i j}\right)$ dada em (3.1), $\operatorname{Corr}\left(\mathbf{u}_{i}\right)$ é a ver- 
dadeira matriz de correlação entre $u_{i 1}, \ldots, u_{i s}, \boldsymbol{\Sigma}_{i}=\operatorname{diag}\left\{\operatorname{Var}\left(u_{i 1}\right), \ldots, \operatorname{Var}\left(u_{i s}\right)\right\}$, com $\operatorname{Var}\left(u_{i j}\right)$ dada em D.2, $\mathbf{G}_{i}=\operatorname{diag}\left\{\partial g^{-1}\left(\mathbf{z}_{i 1}^{\top} \boldsymbol{\gamma}\right) / \partial \delta_{i 1}, \ldots, \partial g^{-1}\left(\mathbf{z}_{i s}^{\top} \boldsymbol{\gamma}\right) / \partial \delta_{i s}\right\}, \mathbf{M}_{i}=\operatorname{diag}\left\{\mathrm{E}\left(\dot{v}_{i 1}\right), \ldots, \mathrm{E}\left(\dot{v}_{i s}\right)\right\}$ com $\mathrm{E}\left(\dot{v}_{i j}\right)$ dada em 4.13, $\operatorname{Corr}\left(\mathbf{v}_{i}\right)$ é a verdadeira matriz de correlação, $\boldsymbol{\Omega}_{i}=\operatorname{diag}\left\{\operatorname{Var}\left(u_{i 1}\right), \ldots, \operatorname{Var}\left(u_{i s}\right)\right\}, \operatorname{com} \operatorname{Var}\left(v_{i j}\right)$ dada em (4.15).

Com o objetivo de diminuir o número de parâmetros de perturbação assumimos que as equações de estimação são independentes, isto é, $\operatorname{Cov}\left(\mathbf{u}_{\mathbf{i}}, \mathbf{v}_{\mathbf{i}}\right)=\mathbf{0}$; e que não há dependência entre os elementos de $\mathbf{v}_{i}$, ou seja, $\operatorname{Corr}\left(\mathbf{v}_{i}\right)=\mathbf{I}_{\mathbf{s}}$.

A função de estimação generalizada proposta para $\boldsymbol{\theta}$ é dada então por,

$$
\begin{aligned}
\boldsymbol{\Gamma}_{n}(\boldsymbol{\theta}) & =\left(\begin{array}{c}
\boldsymbol{\Gamma}_{n}(\boldsymbol{\beta}) \\
\boldsymbol{\Gamma}_{n}(\boldsymbol{\alpha})
\end{array}\right)=\sum_{i=1}^{n}\left(\begin{array}{c}
\mathbf{X}_{i}^{\top} \mathbf{N}_{i}\left(\boldsymbol{\Sigma}_{i}^{1 / 2} \mathbf{R}_{i}(\rho) \boldsymbol{\Sigma}_{i}^{1 / 2}\right)^{-1} \mathbf{u}_{i} \\
\mathbf{Z}_{i}^{\top} \mathbf{H}_{i} \mathbf{M}_{i} \boldsymbol{\Omega}_{i}^{-1} \mathbf{v}_{i}
\end{array}\right) \\
& =\sum_{i=1}^{n} \mathbf{Q}_{i}^{\top} \boldsymbol{\Lambda}_{i} \mathbf{\Upsilon}_{i}^{-1} \mathbf{d}_{i}
\end{aligned}
$$

sendo $\mathbf{Q}_{i}=\left(\begin{array}{cc}\mathbf{X}_{i} & \mathbf{0} \\ \mathbf{0} & \mathbf{Z}_{i}\end{array}\right), \boldsymbol{\Lambda}_{i}=\left(\begin{array}{cc}\mathbf{N}_{i} & \mathbf{0} \\ \mathbf{0} & \mathbf{H}_{i} \mathbf{M}_{i}\end{array}\right)$ e $\boldsymbol{\Upsilon}_{i}=\left(\begin{array}{cc}\boldsymbol{\Sigma}_{i}^{1 / 2} \mathbf{R}_{i}(\rho) \boldsymbol{\Sigma}_{i}^{1 / 2} & \mathbf{0} \\ \mathbf{0} & \boldsymbol{\Omega}_{i}\end{array}\right)$.

\subsubsection{Algoritmo de estimação}

Para encontrarmos o estimador $\hat{\boldsymbol{\theta}}$ de $\boldsymbol{\theta}$, devemos resolver a seguinte equação de estimação:

$$
\boldsymbol{\Gamma}_{n}(\hat{\boldsymbol{\theta}})=0
$$

ou, equivalentemente,

$$
\sum_{i=1}^{n} \mathbf{Q}_{\mathbf{i}}^{\top} \boldsymbol{\Lambda}_{i} \mathbf{\Upsilon}_{i}^{-1} \mathbf{d}_{i}=0
$$

Aplicando-se o método de Newton-Raphson chegou-se ao seguinte processo iterativo separadamente a cada passo do processo iterativo. Newton-Raphson, expandindo a EEG dada na equação (4.19) em torno de um valor inicial $\boldsymbol{\theta}^{(0)}$

$$
\boldsymbol{\theta}^{(m+1)}=\boldsymbol{\theta}^{(m)}-\left[\frac{\partial}{\partial \boldsymbol{\theta}^{\top}} \boldsymbol{\Gamma}\left(\boldsymbol{\theta}^{(m)}\right)\right]^{-1} \boldsymbol{\Gamma}\left(\boldsymbol{\theta}^{(m)}\right),
$$

sendo $m$ o número de iterações. $\mathrm{O}$ índice $\mathrm{m}$ no lado direito das equações acima indica que as matrizes e os vetores são atualizados pelas estimativas de $\boldsymbol{\beta}, \boldsymbol{\alpha}, \rho$ da $m$-ésima iteração.

Para estimar $\rho$, procedemos como descrito na Seção 2.2.2.

O processo iterativo é desenvolvido a partir das seguintes etapas: 


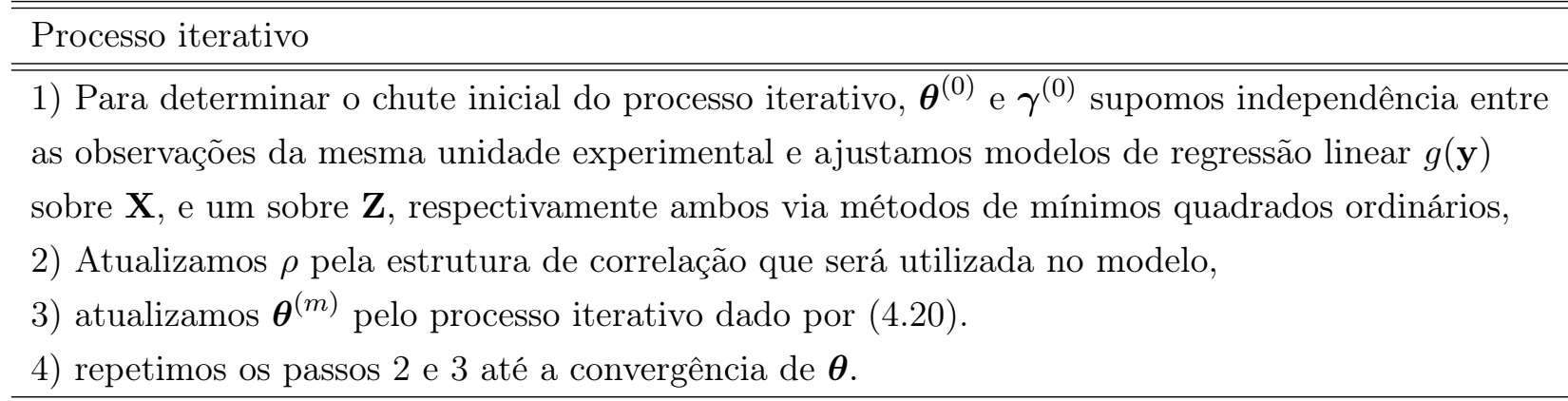

\subsubsection{Estudo de Simulação}

Seguiremos nessa sessão a metodologia estabelecida em 4.1.4. Agora iremos analisar dados logBS-t correlacionados heterogêneos. Temos que os componentes de $\boldsymbol{\mu}_{i}$ são modelados por

$$
\mu_{i j}=\beta_{0}+\beta_{1} x_{i j}
$$

em que $x_{i j}$ 's denotam valores fixos gerados de uma distribuição uniforme no intervalo $[0,1]$ e $\beta_{0}=4$, $\beta_{1}=-2$ e $\nu=3$ e $\nu=7$. Para introduzir o parâmetro de forma variável, os componentes de $\boldsymbol{\alpha}_{i}$ são modelados como

$$
\alpha_{i j}=\exp \left(\gamma_{0}+\gamma_{1} z_{i j}\right)
$$

em que $z_{i j}$ 's denotam valores fixos gerados de distribuição uniforme no intervalo $[0,1] \operatorname{com}\left(\gamma_{0}, \gamma_{1}\right)=$ $(2,-2)$ gerando assim grau de heterogeneidade aproximadamente 7 , e distribuição uniforme no intervalo $[0,1.4] \operatorname{com}\left(\gamma_{0}, \gamma_{1}\right)=(3,-3)$ resultando em grau de heterogeneidade de aproximadamente 60.

Assim como nos demais estudos de simulação vamos considerar $\rho=0.3,0.6$ e 0.9 , tamanhos amostrais $n=10,20,50$ e 80 e $s=3,5$ e 10 . O viés relativo (VR) de $\theta_{j}$ é estimado como $100 \times \mid \bar{\theta}_{j}-$ $\theta_{j} \mid / \theta_{j}, \operatorname{com} \bar{\theta}_{j}=R^{-1} \sum_{r=1}^{R} \hat{\theta}_{j}^{(r)}$, com $\theta_{j}=\left(\beta_{0}, \beta_{1}, \gamma_{0}, \gamma_{1}\right), \hat{\theta}_{j}^{(r)}$ sendo a estimativa EEG de $\theta_{j}$ na $r$-ésima réplica, para $j=0,1, \ldots, R$, e o erro quadrático médio (EQM) é dado por $R^{-1} \sum_{r=1}^{R}\left(\theta_{j}-\right.$ $\left.\hat{\beta}_{j}^{(r)}\right)^{2}$. Consideramos para cada caso $R=5000$ réplicas.

Podemos observar também que os erros quadráticos médios das estimativas de $\gamma_{0}$ e $\gamma_{1}$ diminuem quando as amostras crescem e as demais características são fixas (correlação e tamanho grupo), o mesmo ocorre quando fixamos o tamanho amostral e a correlação e comparamos e o grupo aumenta, ou quando fixamos o tamanho do grupo e o tamanho amostral e quem cresce é a correlação. Podemos ainda observar que quando o grau de heterogeneidade é maior, os EQM's são menores.

\subsubsection{Métodos de diagnóstico}

Assim como na Seção 3.2.6 o procedimento de diagnóstico é similar ao que foi feito na Seção 4.1.5, porém é necessário que sejam realizadas adaptações para a obtenção da influência local. Portanto, apresentaremos aqui apenas a matriz $\Delta$ para a influência local para os esquemas: perturbação da variável resposta e perturbação individual das covariáveis.

i) Perturbação da variável resposta 
Considerando a perturbação da variável resposta

$$
y_{\omega i j}=y_{i j}+\omega_{i j} s_{y_{i j}}
$$

temos que

$$
\boldsymbol{\Gamma}(\boldsymbol{\theta} \mid \boldsymbol{\omega})=\mathbf{Q}^{\top} \mathbf{W} \boldsymbol{\Lambda}^{-1} \mathbf{d}_{\omega}
$$

em que $\mathbf{d}_{\omega}=\left(\mathbf{d}_{\omega 1}^{\top}, \ldots, \mathbf{d}_{\omega n}^{\top}\right)^{\top}, \operatorname{com} \mathbf{d}_{\omega i}=\left(d_{\omega i 1}^{\top}, \ldots, d_{\omega i s}^{\top}\right)^{\top}$,

$$
\boldsymbol{\Delta}=\frac{\partial \boldsymbol{\Gamma}(\boldsymbol{\theta} \mid \boldsymbol{\omega})}{\partial \boldsymbol{\omega}^{\top}}=\mathbf{Q}^{\top} \mathbf{W} \boldsymbol{\Lambda}^{-1} \frac{\partial \mathbf{d}_{\omega}}{\partial \boldsymbol{\omega}^{\top}}
$$

avaliado em $\hat{\boldsymbol{\theta}}$ e em $\boldsymbol{\omega}_{0}$. O vetor perturbado na variável resposta para a $i$-ésima unidade experimental é $\mathbf{d}_{\omega i}=\left(\mathbf{u}_{\omega i}^{\top}, \mathbf{v}_{\omega_{i}}^{\top}\right)^{\top}$, em que $\mathbf{u}_{\omega i}=\left(u_{\omega i 1}, \ldots, u_{\omega i s}\right)^{\top}$ e $\mathbf{v}_{\omega i}=\left(v_{\omega i 1}, \ldots, v_{\omega i s}\right)^{\top}$, com $u_{\omega i j}$ definido em (3.8) com derivada em relação a $\omega_{i j}$ dada em (3.9).

E temos que,

$$
v_{\omega i j}=\frac{1}{\alpha_{i j}}\left[4 w_{\omega i j} \xi_{\omega i j 2}^{2}-1\right]
$$

em que $w_{\omega i j}=\frac{\nu+1}{\nu+4 \xi_{\omega i j 2}^{2}}$ e $\xi_{\omega i j 2}=\frac{1}{\alpha} \operatorname{senh}\left(\frac{y_{\omega i j}-\mu_{i j}}{2}\right)$ e temos que,

$$
\frac{\partial v_{\omega_{i j}}}{\partial \omega_{i j}}=s_{y_{i j}} \frac{4}{\alpha} w_{\omega i j} \xi_{\omega i j 2} \xi_{\omega i j 1}\left[1-\frac{\xi_{\omega i j 2}^{2}}{\nu+4 \xi_{\omega i j 2}^{2}}\right],
$$

em que $\xi_{\omega i j 1}=\frac{1}{\alpha} \cosh \left(\frac{y_{\omega i j}-\mu_{i j}}{2}\right)$. Portanto,

$$
\frac{\partial \mathbf{d}}{\partial \boldsymbol{\omega}^{\top}}=\left(\begin{array}{c}
\dot{\mathcal{U}} \\
\dot{\mathcal{V}}
\end{array}\right)
$$

$\operatorname{com} \dot{\mathcal{U}}=\operatorname{diag}\left\{\dot{\mathcal{U}}_{1}, \ldots, \dot{\mathcal{U}}_{n}\right\}$ e $\dot{\mathcal{V}}=\operatorname{diag}\left\{\dot{\mathcal{V}}_{1}, \ldots, \dot{\mathcal{V}}_{n}\right\}$ em que $\dot{\mathcal{U}}_{i}=\operatorname{diag}\left\{\frac{\partial u_{\omega i 1}}{\partial \omega_{i 1}}, \ldots, \frac{\partial u_{\omega i s}}{\partial \omega_{i s}}\right\}$ e $\dot{\mathcal{V}}_{i}=$ $\operatorname{diag}\left\{\frac{\partial v_{\omega i 1}}{\partial \omega_{i 1}}, \ldots, \frac{\partial v_{\omega i s}}{\partial \omega_{i s}}\right\}$. 


\section{Capítulo 5}

\section{Considerações Finais}

Neste trabalho derivamos equações de estimação generalizadas para a análise de dados log-BS e log-BS-t correlacionados, cujas raízes levam a estimadores consistentes e assintoticamente normais. Alguns procedimentos usuais de diagnóstico foram desenvolvidos de modo que fosse possível averiguar a adequação dos modelos propostos através de análise de resíduos, distância de Cook e influência local. Este último procedimento sob três esquemas de perturbação: ponderação de casos, perturbação da variável resposta e perturbação individual das covariáveis.

Estudos de simulação foram conduzidos para avaliar as propriedades empíricas dos estimadores. Assim como, para avaliar a distribuição dos resíduos. Pudemos assim averiguar que o método proposto para a análise de dados BS (ou BS-t) correlacionados leva a estimativas eficientes. Destacamos que as estimativas dos parâmetros de regressão permanecem consistentes mesmo diante de erros na especificação da estrutura de correlação.

Dados reais foram analisados com o intuito de ilustrar os procedimentos discutidos durante a tese. Quando consideramos o banco de dados referente à produtividade de capital público dos 48 estados norte-americanos contíguos de 1970 a 1986, concluímos ser razoável assumir que marginalmente o PIB assume distribuição $\operatorname{BS}\left(\alpha, \eta_{i j}\right)$, em que $\eta_{i j}$ é explicado pelo Capital de Saneamento Básico. Já no estudo realizado na Escola de Educação Física e Esporte da Universidade de São Paulo (USP), concluímos ser razoável admitir que marginalmente o máximo do primeiro pico de força assume distribuição log-BS-t $\left(\alpha, \eta_{i j}, \nu\right)$, em que $\eta_{i j}$ é explicado pelas variáveis: condição (calçado ou descalço), grupo (experimental ou controle), momento (inicial, 3 meses, ou 6 meses) e ainda pela interação entre grupo e momento.

A metodologia proposta e discutida nesta tese foi toda implementada no ambiente R.

\subsection{Trabalhos futuros}

- fazer um estudo comparativo entre diferentes métodos para analisar dados Birnbaum-Saunders correlacionados;

- incluir o método de EEGs para estudar a estrutura de correlação;

- incluir estrutura de correlação para o modelo do parâmetro de forma, $\alpha$. 


\section{Apêndice A}

Vamos obter uma função $u(\cdot)$ tal que,

$$
\mathrm{E}_{\mu_{i j}}\left\{u_{i j}\left(y_{i j}, \mu_{i j}\right)\right\}=0 .
$$

A função escore do parâmetro $\mu_{i j}$ é dada por,

$$
u_{i j}=-\frac{1}{2} \operatorname{tgh}\left(\frac{y_{i j}-\mu_{i j}}{2}\right)+\frac{1}{\alpha^{2}} \operatorname{senh}\left(y_{i j}-\mu_{i j}\right) .
$$

Note que,

$$
u_{i j}=\frac{\partial l\left(\mu_{i j} ; y_{i j}\right)}{\partial \mu_{i j}}=\frac{\partial \log f\left(y_{i j}, \mu_{i j}\right)}{\partial \mu_{i j}}=\frac{1}{f\left(y_{i j}\right)} \frac{\partial f\left(y_{i j}\right)}{\partial \mu_{i j}} .
$$

Portanto,

$$
\begin{aligned}
\mathrm{E}_{\mu_{i j}}\left\{u_{i j}\left(y_{i j}, \mu_{i j}\right)\right\} & =\int_{-\infty}^{\infty} u_{i j}\left(y_{i j}, \mu_{i j}\right) f\left(y_{i j}, \mu_{i j}\right) d y_{i j}=\int_{-\infty}^{\infty} \frac{1}{f\left(y_{i j}\right)} \frac{\partial f\left(y_{i j}\right)}{\partial \mu_{i j}} f\left(y_{i j}, \mu_{i j}\right) d y_{i j} \\
& =\int_{-\infty}^{\infty} \frac{\partial f\left(y_{i j}, \mu_{i j}\right)}{\partial \mu_{i j}} d y_{i j} .
\end{aligned}
$$

Observe que,

$$
\begin{aligned}
\frac{\partial f\left(y_{i j}, \mu_{i j}\right)}{\partial \mu_{i j}}= & \left(\frac{1}{\alpha \sqrt{2 \pi}}\right) \cosh \left(\frac{y_{i j}-\mu_{i j}}{2}\right) \exp \left\{-2 \alpha^{2} \operatorname{senh}^{2}\left(\frac{y_{i j}-\mu_{i j}}{2}\right)\right\} \\
& \left\{-2 \alpha^{-2} 2 \operatorname{senh}\left(\frac{y_{i j}-\mu_{i j}}{2}\right) \cosh \left(\frac{y_{i j}-\mu_{i j}}{2}\right)\left(\frac{-1}{2}\right)\right\} \\
& +\left(\frac{1}{\alpha \sqrt{2 \pi}}\right) \exp \left\{-2 \alpha^{2} \operatorname{senh}^{2}\left(\frac{y_{i j}-\mu_{i j}}{2}\right)\right\} \operatorname{senh}\left(\frac{\left(y_{i j}-\mu_{i j}\right)}{2}\right)\left(-\frac{1}{2}\right) \\
= & \frac{1}{\alpha \sqrt{2 \pi}} \operatorname{senh}\left(\frac{y_{i j}-\mu_{i j}}{2}\right) \exp \left\{-2 \alpha^{2} \operatorname{senh}^{2}\left(\frac{y_{i j}-\mu_{i j}}{2}\right)\right\} \\
& \left\{\cosh ^{2}\left(\frac{y_{i j}-\mu_{i j}}{2} 2 \alpha^{-2}-\frac{1}{2}\right)\right\} .
\end{aligned}
$$

Temos acima o produto de funções contínuas para todo $y_{i j} \in \mathbb{R}$, portanto também é contínua. De acordo com a Regra de Leibniz pode-se derivar sob o sinal da integral desde que o integrando resultante seja uma função contínua. Mais detalhes em Lages (2000, pg 143). 
Como, $\frac{\partial f\left(y_{i j}, \mu_{i j}\right)}{\partial \mu_{i j}}$ é uma função contínua segue que,

$$
\mathrm{E}_{\mu_{i j}}\left\{u_{i j}\left(y_{i j}, \mu_{i j}\right)\right\}=\int_{-\infty}^{\infty} \frac{\partial f\left(y_{i j}, \mu_{i j}\right)}{\partial \mu_{i j}} d y_{i j}=\frac{\partial}{\partial \mu_{i j}} \int_{-\infty}^{\infty} f\left(y_{i j}, \mu_{i j}\right) d y_{i j}=0 .
$$




\section{Apêndice B}

\section{Cálculos do Capítulo 2}

\section{B.1 Função de estimação para o parâmetro de localização}

Iremos apresentar nesta seção os cálculos detalhados para a obtenção da função de estimação ótima descrita em (2.4). Temos que

$$
u_{i j}=-\frac{1}{2} \tanh \left(\frac{y_{i j}-\mu_{i j}}{2}\right)+\frac{1}{\alpha^{2}} \operatorname{senh}\left(y_{i j}-\mu_{i j}\right) .
$$

Então,

$$
\begin{aligned}
\dot{u}_{i j} & =\frac{\partial u_{i j}}{\partial \mu_{i j}}=\frac{1}{4}\left[1-\tanh ^{2}\left(\frac{y_{i j}-\mu_{i j}}{2}\right)\right]-\frac{1}{\alpha^{2}} \cosh \left(y_{i j}-\mu_{i j}\right) \\
& =\frac{1}{4} \operatorname{sech}^{2}\left(\frac{y_{i j}-\mu_{i j}}{2}\right)-\frac{1}{\alpha^{2}} \cosh \left(y_{i j}-\mu_{i j}\right) \\
& =\frac{1}{4} \operatorname{sech}^{2}\left(\frac{y_{i j}-\mu_{i j}}{2}\right)-\frac{1}{\alpha^{2}}\left[\cosh ^{2}\left(\frac{y_{i j}-\mu_{i j}}{2}\right)+\operatorname{senh}^{2}\left(\frac{y_{i j}-\mu_{i j}}{2}\right)\right] \\
& =\frac{1}{4} \operatorname{sech}^{2}\left(\frac{y_{i j}-\mu_{i j}}{2}\right)-\frac{1}{\alpha^{2}}\left[1+\operatorname{senh}^{2}\left(\frac{y_{i j}-\mu_{i j}}{2}\right)+\operatorname{senh}^{2}\left(\frac{y_{i j}-\mu_{i j}}{2}\right)\right] \\
& =\frac{1}{4} \operatorname{sech}^{2}\left(\frac{y_{i j}-\mu_{i j}}{2}\right)-\frac{1}{\alpha^{2}}\left[1+2 \operatorname{senh}^{2}\left(\frac{y_{i j}-\mu_{i j}}{2}\right)\right] .
\end{aligned}
$$

Logo,

$$
\begin{aligned}
\mathrm{E}\left(\dot{u}_{i j}\right) & =\mathrm{E}\left\{\frac{1}{4} \operatorname{sech}^{2}\left(\frac{y_{i j}-\mu_{i j}}{2}\right)-\frac{1}{\alpha^{2}}\left[1+2 \operatorname{senh}^{2}\left(\frac{y_{i j}-\mu_{i j}}{2}\right)\right]\right\} \\
& =\mathrm{E}\left\{\frac{1}{4} \operatorname{sech}^{2}\left(\frac{y_{i j}-\mu_{i j}}{2}\right)\right\}-\frac{1}{\alpha^{2}}-\frac{2}{\alpha^{2}} \mathrm{E}\left\{\operatorname{senh}^{2}\left(\frac{y_{i j}-\mu_{i j}}{2}\right)\right\} .
\end{aligned}
$$

Observe que

$$
\begin{aligned}
\mathrm{E}\left\{\operatorname{senh}^{2}\left(\frac{y_{i j}-\mu_{i j}}{2}\right)\right\}= & \int_{-\infty}^{\infty} \operatorname{senh}^{2}\left(\frac{y_{i j}-\mu_{i j}}{2}\right) \frac{1}{\alpha \sqrt{2 \pi}} \cosh \left(\frac{y_{i j}-\mu_{i j}}{2}\right) \\
& \times \exp \left[-2\left\{\frac{1}{\alpha} \operatorname{senh}\left(\frac{y_{i j}-\mu_{i j}}{2}\right)\right\}^{2}\right] d y_{i j} .
\end{aligned}
$$


Fazendo a substituição $a=\operatorname{senh}\left(\frac{y_{i j}-\mu_{i j}}{2}\right), 2 d a=\cosh \left(\frac{y_{i j}-\mu_{i j}}{2}\right) d y_{i j}$

$$
\begin{aligned}
\mathrm{E}\left\{\sinh ^{2}\left(\frac{y_{i j}-\mu_{i j}}{2}\right)\right\} & =\int_{-\infty}^{\infty} \frac{2}{\sqrt{2 \pi} \alpha} a^{2} \exp \left[-2\left(\frac{a}{\alpha}\right)^{2}\right] d a \\
& =\int_{-\infty}^{\infty} \frac{1}{\sqrt{2 \pi} \alpha / 2} a^{2} \exp \left(-\frac{a^{2}}{2 \alpha^{2} / 4}\right) d a=\frac{\alpha^{2}}{4}
\end{aligned}
$$

que é o segundo momento central da distribuição normal com média 0 e variância $\left(\alpha^{2} / 4\right)$.

Portanto,

$$
\begin{aligned}
\mathrm{E}\left(\dot{u}_{i j}\right) & =\mathrm{E}\left\{\frac{1}{4} \operatorname{sech}^{2}\left(\frac{y_{i j}-\mu_{i j}}{2}\right)\right\}-\frac{1}{\alpha^{2}}-\frac{2}{\alpha^{2}} \times \frac{\alpha^{2}}{4} \\
& =\frac{1}{4} \mathrm{E}\left\{\operatorname{sech}^{2}\left(\frac{y_{i j}-\mu_{i j}}{2}\right)\right\}-\frac{1}{2}-\frac{1}{\alpha^{2}} .
\end{aligned}
$$

Denotamos,

$$
\mathbf{N}_{i}=\mathrm{E}\left(\frac{\partial \mathbf{u}_{i}}{\partial \boldsymbol{\mu}_{i}}\right)=\operatorname{diag}\left\{\mathrm{E}\left(\dot{u}_{i 1}\right), \ldots, \mathrm{E}\left(\dot{u}_{i s}\right)\right\}
$$

então,

$$
\begin{gathered}
\frac{\partial \mathbf{u}_{i}}{\partial \boldsymbol{\mu}_{i}} \frac{\partial \boldsymbol{\mu}_{i}}{\partial \boldsymbol{\eta}_{i}} \frac{\partial \boldsymbol{\eta}_{i}}{\partial \boldsymbol{\beta}^{\top}}=\mathbf{N}_{i} \mathbf{X}_{i} \\
\mathrm{E}\left(\frac{\partial \mathbf{u}_{i}}{\partial \boldsymbol{\beta}^{\top}}\right)^{\top}=\mathrm{E}\left(\frac{\partial \mathbf{u}_{i}}{\partial \boldsymbol{\mu}_{i}} \frac{\partial \boldsymbol{\mu}_{i}}{\partial \boldsymbol{\eta}_{i}} \frac{\partial \boldsymbol{\eta}_{i}}{\partial \boldsymbol{\beta}^{\top}}\right)^{\top}=\mathbf{X}_{i}^{\top} \mathbf{N}_{i} .
\end{gathered}
$$

A matriz de variância-covariâncias de $\mathbf{u}_{i}$ pode ser escrita na forma

$$
\operatorname{Cov}\left(\mathbf{u}_{i}\right)=\operatorname{Var}\left(\mathbf{u}_{i}\right)^{1 / 2} \operatorname{Corr}\left(\mathbf{u}_{i}\right) \operatorname{Var}\left(\mathbf{u}_{i}\right)^{1 / 2},
$$

em que $\operatorname{Corr}\left(\mathbf{u}_{i}\right)$ denota a matriz de correlação de $\mathbf{u}_{i}$ e $\operatorname{Var}\left(\mathbf{u}_{i}\right)$ é dada por,

$$
\operatorname{Var}\left(\mathbf{u}_{i}\right)=\operatorname{diag}\left\{\operatorname{Var}\left(u_{i 1}\right), \ldots, \operatorname{Var}\left(u_{i s}\right)\right\}=\operatorname{diag}\left\{\mathrm{E}\left(u_{i 1}^{2}\right), \ldots, \mathrm{E}\left(u_{i s}^{2}\right)\right\}=\boldsymbol{\Sigma}_{i}
$$

em que,

$$
\begin{aligned}
u_{i j}^{2}= & {\left[-\frac{1}{2} \operatorname{tgh}\left(\frac{y_{i j}-\mu_{i j}}{2}\right)+\frac{1}{\alpha^{2}} \operatorname{senh}\left(y_{i j}-\mu_{i j}\right)\right]^{2} } \\
= & \frac{1}{4} \operatorname{tgh}^{2}\left(\frac{y_{i j}-\mu_{i j}}{2}\right)-\frac{1}{\alpha^{2}} \operatorname{tgh}\left(\frac{y_{i j}-\mu_{i j}}{2}\right) \operatorname{senh}\left(y_{i j}-\mu_{i j}\right)+\frac{1}{\alpha^{4}} \operatorname{senh}^{2}\left(y_{i j}-\mu_{i j}\right) \\
= & \frac{1}{4} \operatorname{tgh}^{2}\left(\frac{y_{i j}-\mu_{i j}}{2}\right)-\frac{1}{\alpha^{2}} \operatorname{tgh}\left(\frac{y_{i j}-\mu_{i j}}{2}\right) 2 \operatorname{senh}\left(\frac{y_{i j}-\mu_{i j}}{2}\right) \cosh \left(\frac{y_{i j}-\mu_{i j}}{2}\right) \\
& +\frac{1}{\alpha^{4}} \operatorname{senh}^{2}\left(y_{i j}-\mu_{i j}\right) \\
= & \frac{1}{4} \operatorname{tgh}^{2}\left(\frac{y_{i j}-\mu_{i j}}{2}\right)-\frac{2}{\alpha^{2}} \operatorname{senh}^{2}\left(\frac{y_{i j}-\mu_{i j}}{2}\right)+\frac{1}{\alpha^{4}} \operatorname{senh}^{2}\left(y_{i j}-\mu_{i j}\right)
\end{aligned}
$$




$$
\begin{aligned}
= & \frac{1}{4} \operatorname{tgh}^{2}\left(\frac{y_{i j}-\mu_{i j}}{2}\right)-\frac{2}{\alpha^{2}} \operatorname{senh}^{2}\left(\frac{y_{i j}-\mu_{i j}}{2}\right)+\frac{1}{\alpha^{4}}\left[2 \operatorname{senh}\left(\frac{y_{i j}-\mu_{i j}}{2}\right) \cosh \left(\frac{y_{i j}-\mu_{i j}}{2}\right)\right]^{2} \\
= & \frac{1}{4} \operatorname{tgh}^{2}\left(\frac{y_{i j}-\mu_{i j}}{2}\right)-\frac{2}{\alpha^{2}} \operatorname{senh}^{2}\left(\frac{y_{i j}-\mu_{i j}}{2}\right)+\frac{1}{\alpha^{4}} 4 \operatorname{senh}^{2}\left(\frac{y_{i j}-\mu_{i j}}{2}\right) \cosh ^{2}\left(\frac{y_{i j}-\mu_{i j}}{2}\right) \\
= & \frac{1}{4} \operatorname{tgh}^{2}\left(\frac{y_{i j}-\mu_{i j}}{2}\right)-\frac{2}{\alpha^{2}} \operatorname{senh}^{2}\left(\frac{y_{i j}-\mu_{i j}}{2}\right) \frac{1}{\alpha^{4}} 4 \operatorname{senh}^{2}\left(\frac{y_{i j}-\mu_{i j}}{2}\right) \\
& \times\left(1+\operatorname{senh}^{2}\left(\frac{y_{i j}-\mu_{i j}}{2}\right)\right) \\
= & \frac{1}{4} \operatorname{tgh}^{2}\left(\frac{y_{i j}-\mu_{i j}}{2}\right)-\frac{2}{\alpha^{2}} \operatorname{senh}^{2}\left(\frac{y_{i j}-\mu_{i j}}{2}\right)+\frac{1}{\alpha^{4}} 4 \operatorname{senh}^{2}\left(\frac{y_{i j}-\mu_{i j}}{2}\right) \\
& +\frac{1}{\alpha^{4}} 4 \operatorname{senh}^{4}\left(\frac{y_{i j}-\mu_{i j}}{2}\right),
\end{aligned}
$$

e

$$
\begin{aligned}
\mathrm{E}\left(u_{i j}^{2}\right)= & \mathrm{E}\left\{\frac{1}{4} \operatorname{tgh}^{2}\left(\frac{y_{i j}-\mu_{i j}}{2}\right)-\frac{2}{\alpha^{2}} \operatorname{senh}^{2}\left(\frac{y_{i j}-\mu_{i j}}{2}\right)+\frac{1}{\alpha^{4}} 4 \operatorname{senh}^{2}\left(\frac{y_{i j}-\mu_{i j}}{2}\right)\right. \\
& \left.\frac{1}{\alpha^{4}} 4 \operatorname{senh}^{4}\left(\frac{y_{i j}-\mu_{i j}}{2}\right)\right\} \\
= & \mathrm{E}\left\{\frac{1}{4} \operatorname{tgh}^{2}\left(\frac{y_{i j}-\mu_{i j}}{2}\right)\right\}-\frac{2}{\alpha^{2}} \mathrm{E}\left\{\operatorname{senh}^{2}\left(\frac{y_{i j}-\mu_{i j}}{2}\right)\right\}+\frac{4}{\alpha^{4}} \mathrm{E}\left\{\operatorname{senh}^{2}\left(\frac{y_{i j}-\mu_{i j}}{2}\right)\right\} \\
& +\frac{4}{\alpha^{4}} \mathrm{E}\left\{\operatorname{senh}^{4}\left(\frac{y_{i j}-\mu_{i j}}{2}\right)\right\} .
\end{aligned}
$$

Analogamente a (B.1) temos que,

$$
\begin{aligned}
\mathrm{E}\left(\sinh ^{4}\left(\frac{y_{i j}-\mu_{i j}}{2}\right)\right) & =\int_{-\infty}^{\infty} \frac{2}{\sqrt{2 \pi} \alpha} a^{4} \exp \left[-2\left(\frac{a}{\alpha}\right)^{2}\right] d a \\
& =\int_{-\infty}^{\infty} \frac{1}{\sqrt{2 \pi} \alpha / 2} a^{4} \exp \left(-\frac{a^{2}}{2 \alpha^{2} / 4}\right) d a=\frac{3 \alpha^{4}}{16}
\end{aligned}
$$

Portanto, aplicando (B.1) e (B.3) em (B.2) segue que

$$
\begin{aligned}
\mathrm{E}\left(u_{i j}^{2}\right) & =\mathrm{E}\left\{\frac{1}{4} \operatorname{tgh}^{2}\left(\frac{y_{i j}-\mu_{i j}}{2}\right)\right\}-\frac{2}{\alpha^{2}} \times \frac{\alpha^{2}}{4}+\frac{4}{\alpha^{4}} \times \frac{\alpha^{2}}{4}+\frac{4}{\alpha^{4}} \times \frac{3 \alpha^{4}}{16} \\
& =\mathrm{E}\left\{\frac{1}{4} \tanh ^{2}\left(\frac{y_{i j}-\mu_{i j}}{2}\right)\right\}-\frac{1}{2}+\frac{1}{\alpha^{2}}+\frac{3}{4} \\
& =\mathrm{E}\left\{\frac{1}{4} \operatorname{tgh}^{2}\left(\frac{y_{i j}-\mu_{i j}}{2}\right)\right\}+\frac{1}{4}+\frac{1}{\alpha^{2}} .
\end{aligned}
$$

Logo,

$$
\boldsymbol{\Psi}_{n}^{*}(\boldsymbol{\beta})=\sum_{i=1}^{n} \mathbf{X}_{i}^{\top} \mathbf{N}_{i}\left(\boldsymbol{\Sigma}_{i}^{1 / 2} \mathbf{R}\left(\mathbf{u}_{i}\right) \boldsymbol{\Sigma}_{i}^{1 / 2}\right)^{-1} \mathbf{u}_{i}
$$

\section{B.2 Matrizes de sensibilidade e variabilidade}

Iremos derivar nesta seção a matriz de sensibilidade, $\mathbf{S}_{i}(\boldsymbol{\beta})$, e a matriz de variabilidade, $\mathbf{V}_{i}(\boldsymbol{\beta})$, para a função de estimação generalizada (2.4). 
Observe inicialmente que,

$$
\frac{\partial \boldsymbol{\psi}_{i}(\boldsymbol{\beta})}{\partial \boldsymbol{\beta}}=\frac{\partial \boldsymbol{\psi}_{i}(\boldsymbol{\beta})}{\partial \boldsymbol{\mu}_{i}} \times \frac{\partial \boldsymbol{\mu}_{i}}{\partial \boldsymbol{\eta}_{i}} \times \frac{\partial \boldsymbol{\eta}_{i}}{\partial \boldsymbol{\beta}}
$$

Temos que,

$$
\begin{aligned}
\frac{\partial \boldsymbol{\psi}_{i}(\boldsymbol{\beta})}{\partial \boldsymbol{\mu}_{i}}= & \frac{\partial}{\partial \boldsymbol{\mu}_{i}}\left\{\mathbf{X}_{i}^{\top} \mathbf{N}_{i} \boldsymbol{\Sigma}_{i}^{-1 / 2} \mathbf{R}(\boldsymbol{\rho})^{-1} \boldsymbol{\Sigma}_{i}^{-1 / 2} \mathbf{u}_{i}\right\} \\
= & \mathbf{X}_{i}^{\top} \frac{\partial \mathbf{N}_{i}}{\partial \boldsymbol{\mu}_{i}} \boldsymbol{\Sigma}_{i}^{-1 / 2} \mathbf{R}(\boldsymbol{\rho})^{-1} \boldsymbol{\Sigma}_{i}^{-1 / 2} \mathbf{u}_{i}+\mathbf{X}_{i}^{\top} \mathbf{N}_{i} \frac{\partial \boldsymbol{\Sigma}_{i}^{-1 / 2}}{\partial \boldsymbol{\mu}_{i}} \mathbf{R}(\boldsymbol{\rho})^{-1} \boldsymbol{\Sigma}_{i}^{-1 / 2} \mathbf{u}_{i} \\
& +\mathbf{X}_{i}^{\top} \mathbf{N}_{i} \boldsymbol{\Sigma}_{i}^{-1 / 2} \mathbf{R}(\boldsymbol{\rho})^{-1} \frac{\partial \boldsymbol{\Sigma}_{i}^{-1 / 2}}{\partial \boldsymbol{\mu}_{i}} \mathbf{u}_{i}+\mathbf{X}_{i}^{\top} \mathbf{N}_{i} \boldsymbol{\Sigma}_{i}^{-1 / 2} \mathbf{R}(\boldsymbol{\rho})^{-1} \boldsymbol{\Sigma}_{i}^{-1 / 2} \frac{\partial \mathbf{u}_{i}}{\partial \boldsymbol{\mu}_{i}}
\end{aligned}
$$

Note que

$$
\mathrm{E}\left(\dot{u}_{i j}\right)=\frac{1}{4} \mathrm{E}\left\{\operatorname{sech}^{2}\left(\frac{y_{i j}-\mu_{i j}}{2}\right)\right\}-\frac{1}{2}-\frac{1}{\alpha^{2}}
$$

Podemos provar facilmente que $\frac{\partial}{\partial \mu_{i j}}\left[\frac{1}{4} \operatorname{sech}^{2}\left(\frac{y_{i j}-\mu_{i j}}{2}\right) f\left(y_{i j}, \mu_{i j}\right)\right]$ é contínua. Logo, podemos derivar sob o sinal de integração e assim obtemos,

$$
\begin{aligned}
\frac{\partial \mathrm{E}_{\mu_{i j}}\left\{\dot{u}_{i j}\left(y_{i j}, \mu_{i j}\right)\right\}}{\partial \mu_{i j}}= & \frac{\partial}{\partial \mu_{i j}}\left\{\int_{-\infty}^{\infty}\left[\frac{1}{4} \operatorname{sech}^{2}\left(\frac{y_{i j}-\mu_{i j}}{2}\right) f\left(y_{i j}, \mu_{i j}\right)\right] d y_{i j}\right\} \\
= & \int_{-\infty}^{\infty}\left\{\frac{\partial}{\partial \mu_{i j}}\left[\frac{1}{4} \operatorname{sech}^{2}\left(\frac{y_{i j}-\mu_{i j}}{2}\right) f\left(y_{i j}, \mu_{i j}\right)\right]\right\} d y_{i j} \\
= & \int_{-\infty}^{\infty}\left\{\frac{\partial}{\partial \mu_{i j}}\left[\frac{1}{4} \operatorname{sech}^{2}\left(\frac{y_{i j}-\mu_{i j}}{2}\right)\right] \times f\left(y_{i j}, \mu_{i j}\right)\right\} d y_{i j} \\
& +\int_{-\infty}^{\infty}\left\{\frac{1}{4} \operatorname{sech}^{2}\left(\frac{y_{i j}-\mu_{i j}}{2}\right) \times \frac{\partial f\left(y_{i j}, \mu_{i j}\right)}{\partial \mu_{i j}}\right\} d y_{i j} .
\end{aligned}
$$

Observe que,

$$
\begin{aligned}
\frac{\partial f\left(y_{i j}, \mu_{i j}\right)}{\partial \mu_{i j}}= & \frac{1}{\alpha \sqrt{2 \pi}} \operatorname{senh}\left(\frac{y_{i j}-\mu_{i j}}{2}\right)\left(-\frac{1}{2}\right) \exp \left\{-\frac{2}{\alpha^{2}} \operatorname{senh}^{2}\left(\frac{y_{i j}-\mu_{i j}}{2}\right)\right\} \\
& +\frac{1}{\alpha \sqrt{2 \pi}} \cosh \left(\frac{y_{i j}-\mu_{i j}}{2}\right) \exp \left\{-\frac{2}{\alpha^{2}} \operatorname{senh}^{2}\left(\frac{y_{i j}-\mu_{i j}}{2}\right)\right\} \\
& \times\left[\frac{2}{\alpha^{2}} \operatorname{senh}\left(\frac{y_{i j}-\mu_{i j}}{2}\right) \cosh \left(\frac{y_{i j}-\mu_{i j}}{2}\right)\right] \\
= & \frac{1}{\alpha \sqrt{2 \pi}} \operatorname{senh}\left(\frac{y_{i j}-\mu_{i j}}{2}\right) \exp \left\{-\frac{2}{\alpha^{2}} \operatorname{senh}^{2}\left(\frac{y_{i j}-\mu_{i j}}{2}\right)\right\} \\
& \left\{\cosh ^{2}\left(\frac{y_{i j}-\mu_{i j}}{2}\right) \frac{2}{\alpha^{2}}-\frac{1}{2}\right\} .
\end{aligned}
$$

Logo,

$$
\frac{\partial f\left(y_{i j}, \mu_{i j}\right)}{\partial \mu_{i j}}\left[\frac{1}{4} \operatorname{sech}^{2}\left(\frac{y_{i j}-\mu_{i j}}{2}\right)\right]=\frac{1}{\alpha \sqrt{2 \pi}} \operatorname{senh}\left(\frac{y_{i j}-\mu_{i j}}{2}\right) \exp \left\{-\frac{2}{\alpha^{2}} \operatorname{senh}^{2}\left(\frac{y_{i j}-\mu_{i j}}{2}\right)\right\}
$$




$$
\begin{aligned}
& \left\{\cosh ^{2}\left(\frac{y_{i j}-\mu_{i j}}{2}\right) \frac{2}{\alpha^{2}}-\frac{1}{2}\right\}\left\{\frac{1}{4} \operatorname{sech}^{2}\left(\frac{y_{i j}-\mu_{i j}}{2}\right)\right\} \\
= & \frac{1}{\alpha \sqrt{2 \pi}} \operatorname{senh}\left(\frac{y_{i j}-\mu_{i j}}{2}\right) \exp \left\{-\frac{2}{\alpha^{2}} \operatorname{senh}^{2}\left(\frac{y_{i j}-\mu_{i j}}{2}\right)\right\} \\
& \left\{\frac{1}{2 \alpha^{2}}-\frac{1}{8} \operatorname{sech}^{2}\left(\frac{y_{i j}-\mu_{i j}}{2}\right)\right\} \\
= & \frac{1}{\alpha^{3} 2 \sqrt{2 \pi}} \operatorname{senh}\left(\frac{y_{i j}-\mu_{i j}}{2}\right) \exp \left\{-\frac{2}{\alpha^{2}} \operatorname{senh}^{2}\left(\frac{y_{i j}-\mu_{i j}}{2}\right)\right\} \\
& -\frac{1}{\alpha \sqrt{2 \pi}} \operatorname{senh}\left(\frac{y_{i j}-\mu_{i j}}{2}\right) \exp \left\{-\frac{2}{\alpha^{2}} \operatorname{senh}^{2}\left(\frac{y_{i j}-\mu_{i j}}{2}\right)\right\} \\
& \frac{1}{8} \operatorname{sech}^{2}\left(\frac{y_{i j}-\mu_{i j}}{2}\right) .
\end{aligned}
$$

E temos,

$$
\begin{aligned}
& \frac{\partial}{\partial \mu_{i j}}\left[\frac{1}{4} \operatorname{sech}^{2}\left(\frac{y_{i j}-\mu_{i j}}{2}\right)\right] f\left(y_{i j}, \mu_{i j}\right)=\frac{1}{\alpha \sqrt{2 \pi}} \cosh \left(\frac{y_{i j}-\mu_{i j}}{2}\right) \exp \left\{-\frac{2}{\alpha^{2}} \operatorname{senh}^{2}\left(\frac{y_{i j}-\mu_{i j}}{2}\right)\right\} \\
& \left\{-\frac{2}{4} \operatorname{sech}\left(\frac{y_{i j}-\mu_{i j}}{2}\right) \frac{\left[-\operatorname{senh}\left(\frac{y_{i j}-\mu_{i j}}{2}\right)\right]}{\cosh ^{2}\left(\frac{y_{i j}-\mu_{i j}}{2}\right)}\left(-\frac{1}{2}\right)\right\} \\
& =\frac{1}{\alpha \sqrt{2 \pi}} \cosh \left(\frac{y_{i j}-\mu_{i j}}{2}\right) \exp \left\{-\frac{2}{\alpha^{2}} \operatorname{senh}^{2}\left(\frac{y_{i j}-\mu_{i j}}{2}\right)\right\} \\
& \left\{\frac{1}{4} \operatorname{sech}\left(\frac{y_{i j}-\mu_{i j}}{2}\right) \frac{\operatorname{senh}\left(\frac{y_{i j}-\mu_{i j}}{2}\right)}{\cosh ^{2}\left(\frac{y_{i j}-\mu_{i j}}{2}\right)}\right\} \\
& =\frac{1}{\alpha \sqrt{2 \pi}} \operatorname{senh}\left(\frac{y_{i j}-\mu_{i j}}{2}\right) \exp \left\{-\frac{2}{\alpha^{2}} \operatorname{senh}^{2}\left(\frac{y_{i j}-\mu_{i j}}{2}\right)\right\} \\
& \frac{1}{4} \operatorname{sech}^{2}\left(\frac{y_{i j}-\mu_{i j}}{2}\right) \text {. }
\end{aligned}
$$

Portanto, utilizando os resultados obtidos em (B.7) e (B.8), o integrando em (B.5) fica dado por,

$$
\begin{array}{r}
\frac{\partial}{\partial \mu_{i j}}\left[\frac{1}{4} \operatorname{sech}^{2}\left(\frac{y_{i j}-\mu_{i j}}{2}\right)\right] \times f\left(y_{i j}, \mu_{i j}\right)+\frac{1}{4} \operatorname{sech}^{2}\left(\frac{y_{i j}-\mu_{i j}}{2}\right) \times \frac{\partial f\left(y_{i j}, \mu_{i j}\right)}{\partial \mu_{i j}} \\
=\frac{1}{\alpha \sqrt{2 \pi}} \operatorname{senh}\left(\frac{y_{i j}-\mu_{i j}}{2}\right) \exp \left\{-\frac{2}{\alpha^{2}} \operatorname{senh}^{2}\left(\frac{y_{i j}-\mu_{i j}}{2}\right)\right\} \times \frac{1}{2 \alpha^{2}} \\
+\frac{1}{\alpha \sqrt{2 \pi}} \operatorname{senh}\left(\frac{y_{i j}-\mu_{i j}}{2}\right) \exp \left\{-\frac{2}{\alpha^{2}} \operatorname{senh}^{2}\left(\frac{y_{i j}-\mu_{i j}}{2}\right)\right\} \frac{1}{8} \operatorname{sech}^{2}\left(\frac{y_{i j}-\mu_{i 1}}{2}\right) .
\end{array}
$$

Logo, fazendo uma troca de variável, $z_{i j}=y_{i j}-\mu_{i j}$, e depois observando que o integrando resultante é uma função ímpar no intervalo $(-\infty, \infty)$ segue que,

$$
\begin{aligned}
\frac{\partial \mathrm{E}_{\mu_{i j}}\left\{\dot{u}_{i j}\left(y_{i j}, \mu_{i j}\right)\right\}}{\partial \mu_{i j}}= & \int_{-\infty}^{\infty} \frac{\partial}{\partial \mu_{i j}}\left[\frac{1}{4} \operatorname{sech}^{2}\left(\frac{y_{i j}-\mu_{i j}}{2}\right)\right] \times f\left(y_{i j}, \mu_{i j}\right)+\frac{1}{4} \operatorname{sech}^{2}\left(\frac{y_{i j}-\mu_{i j}}{2}\right) \\
& \times \frac{\partial f\left(y_{i j}, \mu_{i j}\right)}{\partial \mu_{i j}} d y_{i j} \\
= & 0 .
\end{aligned}
$$


Portanto,

$$
\frac{\partial \mathbf{N}_{i}}{\partial \boldsymbol{\mu}_{i}}=\mathbf{0}
$$

Temos que,

$$
\mathrm{E}\left(u_{i j}^{2}\right)=\mathrm{E}\left\{\frac{1}{4} \tanh ^{2}\left(\frac{y_{i j}-\mu_{i j}}{2}\right)\right\}+\frac{1}{4}+\frac{1}{\alpha^{2}} .
$$

Podemos provar sem dificuldades que $\frac{\partial}{\partial \mu_{i j}}\left[\frac{1}{4} \operatorname{tgh}^{2}\left(\frac{y_{i j}-\mu_{i j}}{2}\right) f\left(y_{i j}, \mu_{i j}\right)\right]$ é contínua. Logo, podemos derivar sob o sinal de integração e assim obtemos,

$$
\begin{aligned}
\frac{\partial}{\partial \mu_{i j}} \mathrm{E}\left\{\frac{1}{4} \tanh ^{2}\left(\frac{y_{i j}-\mu_{i j}}{2}\right)\right\} & =\frac{\partial}{\partial \mu_{i j}} \int_{-\infty}^{\infty}\left\{f\left(y_{i j}, \mu_{i j}\right) \frac{1}{4} \operatorname{tgh}^{2}\left(\frac{y_{i j}-\mu_{i j}}{2}\right)\right\} d y_{i j} \\
& =\int_{-\infty}^{\infty} \frac{\partial}{\partial \mu_{i j}}\left\{f\left(y_{i j}, \mu_{i j}\right) \frac{1}{4} \operatorname{tgh}^{2}\left(\frac{y_{i j}-\mu_{i j}}{2}\right)\right\} d y_{i j},
\end{aligned}
$$

em que,

$$
\frac{\partial}{\partial \mu_{i j}}\left\{\frac{1}{4} \tanh ^{2}\left(\frac{y_{i j}-\mu_{i j}}{2}\right)\right\} f\left(y_{i j}, \mu_{i j}\right)=-\frac{1}{4} \tanh \left(\frac{y_{i j}-\mu_{i j}}{2}\right) \operatorname{sech}^{2} \underset{\times f\left(y_{i j}, \mu_{i j}\right)}{\left(\frac{y_{i j}-\mu_{i j}}{2}\right)}
$$

e de (B.6),

$$
\begin{aligned}
\frac{\partial f\left(y_{i j}, \mu_{i j}\right)}{\partial \mu_{i j}}\left[\frac{1}{4} \tanh ^{2}\left(\frac{y_{i j}-\mu_{i j}}{2}\right)\right]= & \frac{1}{\alpha \sqrt{2 \pi}} \operatorname{senh}\left(\frac{y_{i j}-\mu_{i j}}{2}\right) \exp \left\{-\frac{2}{\alpha^{2}} \operatorname{senh}^{2}\left(\frac{y_{i j}-\mu_{i j}}{2}\right)\right\} \\
& \left\{\cosh ^{2}\left(\frac{y_{i j}-\mu_{i j}}{2}\right) \frac{2}{\alpha^{2}}-\frac{1}{2}\right\}\left\{\frac{1}{4} \tanh ^{2}\left(\frac{y_{i j}-\mu_{i j}}{2}\right)\right\} \\
= & \frac{1}{\alpha \sqrt{2 \pi}} \operatorname{senh}\left(\frac{y_{i j}-\mu_{i j}}{2}\right) \exp \left\{-\frac{2}{\alpha^{2}} \operatorname{senh}^{2}\left(\frac{y_{i j}-\mu_{i j}}{2}\right)\right\} \\
& \times \frac{1}{2 \alpha^{2}} \sinh ^{2}\left(\frac{y_{i j}-\mu_{i j}}{2}\right)-\frac{1}{\alpha \sqrt{2 \pi}} \operatorname{senh}\left(\frac{y_{i j}-\mu_{i j}}{2}\right) \\
& \exp \left\{-\frac{2}{\alpha^{2}} \operatorname{senh}^{2}\left(\frac{y_{i j}-\mu_{i j}}{2}\right)\right\} \frac{1}{8} \tanh ^{2}\left(\frac{y_{i j}-\mu_{i j}}{2}\right) \\
= & f\left(y_{i j}, \mu_{i j}\right) \frac{1}{2 \alpha^{2}} \sinh ^{2}\left(\frac{y_{i j}-\mu_{i j}}{2}\right) \tanh \left(\frac{y_{i j}-\mu_{i j}}{2}\right) \\
& +f\left(y_{i j}, \mu_{i j}\right) \frac{1}{8} \tanh ^{3}\left(\frac{y_{i j}-\mu_{i j}}{2}\right) .
\end{aligned}
$$

Logo, fazendo uma troca de variável, $x_{i j}=y_{i j}-\mu_{i j}$ e (B.11) e (B.12) em (B.10) e observando que o integrando resultante é uma função ímpar no intervalo $(-\infty, \infty)$, segue que,

$$
\begin{aligned}
\frac{\partial \mathrm{E}_{\mu_{i j}}\left\{u_{i j}^{2}\left(y_{i j}, \mu_{i j}\right)\right\}}{\partial \mu_{i j}}= & \int_{-\infty}^{\infty}\left\{f\left(y_{i j}, \mu_{i j}\right)\left[\frac{1}{2 \alpha^{2}} \sinh ^{2}\left(\frac{y_{i j}-\mu_{i j}}{2}\right) \operatorname{tgh}\left(\frac{y_{i j}-\mu_{i j}}{2}\right)\right]\right. \\
& +f\left(y_{i j}, \mu_{i j}\right) \frac{1}{8} \tanh ^{3}\left(\frac{y_{i j}-\mu_{i j}}{2}\right)
\end{aligned}
$$




$$
\begin{aligned}
& \left.-f\left(y_{i j}, \mu_{i j}\right) \frac{1}{4} \tanh \left(\frac{y_{i j}-\mu_{i j}}{2}\right) \operatorname{sech}^{2}\left(\frac{y_{i j}-\mu_{i j}}{2}\right)\right\} d y_{i j} \\
= & 0 .
\end{aligned}
$$

E ainda temos,

$$
\frac{\partial}{\partial \mu_{i j}}\left\{\mathrm{E}\left(u_{i j}^{2}\right)^{-1 / 2}\right\}=-\frac{1}{2} \frac{1}{\mathrm{E}\left(u_{i j}^{2}\right)^{3 / 2}} \frac{\partial \mathrm{E}\left\{u_{i j}^{2}\left(y_{i j}, \mu_{i j}\right)\right\}}{\partial \mu_{i j}}=0,
$$

de onde segue que,

$$
\frac{\partial \boldsymbol{\Sigma}_{i}^{-1 / 2}}{\partial \boldsymbol{\mu}_{i}}=\mathbf{0}
$$

Portanto, aplicando (B.9) e (B.13) em (B.4) obtemos

$$
\begin{aligned}
\frac{\partial \boldsymbol{\psi}_{i}(\boldsymbol{\beta})}{\partial \boldsymbol{\mu}_{i}} & =\mathbf{X}_{i}^{\top} \mathbf{N}_{i} \boldsymbol{\Sigma}_{i}^{-1 / 2} \mathbf{R}(\boldsymbol{\rho})^{-1} \boldsymbol{\Sigma}_{i}^{-1 / 2} \frac{\partial \mathbf{u}_{i}}{\partial \boldsymbol{\mu}_{i}} \\
& =\mathbf{X}_{i}^{\top} \mathbf{N}_{i} \boldsymbol{\Sigma}_{i}^{-1 / 2} \mathbf{R}(\boldsymbol{\rho})^{-1} \boldsymbol{\Sigma}_{i}^{-1 / 2} \dot{\mathbf{U}}_{i}
\end{aligned}
$$

em que $\dot{\mathbf{U}}_{i}=\operatorname{diag}\left\{\dot{u}_{i j}, \ldots, \dot{u}_{i s}\right\}$.

Logo,

$$
\mathbf{S}_{i}(\boldsymbol{\beta})=\mathrm{E}\left(\frac{\partial \boldsymbol{\psi}_{i}(\boldsymbol{\beta})}{\partial \boldsymbol{\beta}}\right)=\mathbf{X}_{i}^{\top} \mathbf{N}_{i} \boldsymbol{\Sigma}_{i}^{-1 / 2} \mathbf{R}(\boldsymbol{\rho})^{-1} \boldsymbol{\Sigma}_{i}^{-1 / 2} \mathbf{N}_{i} \mathbf{X}_{i}
$$

Temos ainda que,

$$
\begin{aligned}
\mathbf{V}_{i}(\boldsymbol{\beta})=\mathrm{E}\left[\boldsymbol{\psi}_{i}(\boldsymbol{\beta}) \boldsymbol{\psi}_{i}^{\top}(\boldsymbol{\beta})\right] & =\mathrm{E}\left[\mathbf{X}_{i}^{\top} \mathbf{N}_{i} \mathbf{W}_{i}^{-1} \mathbf{u}_{i} \mathbf{u}_{i}^{\top} \mathbf{W}_{i}^{-\top} \mathbf{N}_{i} \mathbf{X}_{i}\right] \\
& =\mathbf{X}_{i}^{\top} \mathbf{N}_{i} \mathbf{W}_{i}^{-1} \mathrm{E}\left[\mathbf{u}_{i} \mathbf{u}_{i}^{\top}\right] \mathbf{W}_{i}^{-\top} \mathbf{N}_{i} \mathbf{X}_{i} \\
& =\mathbf{X}_{i}^{\top} \mathbf{N}_{i} \mathbf{W}_{i}^{-1} \operatorname{Cov}\left(\mathbf{u}_{i}\right) \mathbf{W}_{i}^{-\top} \mathbf{N}_{i} \mathbf{X}_{i} .
\end{aligned}
$$

\section{B.3 Influência conformal sob homogeneidade do parâmetro de forma}

\section{B.3.1 Perturbação da variável resposta}

Observe que

$$
u_{\omega i j}=\frac{1}{\alpha^{2}} \operatorname{senh}\left(y_{\omega i j}-\mu_{i j}\right)-\frac{1}{2} \operatorname{tgh}\left(\frac{y_{\omega i j}-\mu_{i j}}{2}\right),
$$

cuja derivada em relação para $\omega_{i j}$ é dada por,

$$
\begin{aligned}
\frac{\partial u_{\omega i j}}{\partial \omega_{i j}} & =\frac{1}{\alpha^{2}} \cosh \left(y_{\omega i j}-\mu_{i j}\right) \frac{\partial y_{\omega i j}}{\partial \omega_{i j}}-\frac{1}{4} \operatorname{sech}^{2}\left(\frac{y_{\omega i j}-\mu_{i j}}{2}\right) \frac{\partial y_{\omega i j}}{\partial \omega_{i j}} \\
& =s_{y_{i j}}\left[\frac{1}{\alpha^{2}} \cosh \left(y_{\omega i j}-\mu_{i j}\right)-\frac{1}{4} \operatorname{sech}^{2}\left(\frac{y_{\omega i j}-\mu_{i j}}{2}\right)\right]
\end{aligned}
$$




$$
=\dot{\mathcal{U}}_{i j}
$$

\section{B.3.2 Perturbação individual das covariáveis}

Nesta seção temos as contas detalhadas das derivadas de $\mathbf{N}_{\omega i}, \mathbf{C}_{\omega i}$ e $\mathbf{u}_{\omega i}$ com relação à $\boldsymbol{\omega}_{i}^{\top}$. Primeiramente, derivamos $\mathbf{C}_{\omega i}$ com relação à $\boldsymbol{\omega}^{\top}$.

$$
\frac{\partial \mathbf{C}}{\partial \boldsymbol{\omega}^{\top}}=\operatorname{diag}\left\{\frac{\partial \mathbf{C}_{1}}{\partial \boldsymbol{\omega}_{1}^{\top}}, \ldots, \frac{\partial \mathbf{C}_{n}}{\partial \boldsymbol{\omega}_{n}^{\top}}\right)
$$

com

$$
\frac{\partial \mathbf{C}_{i}}{\partial \boldsymbol{\omega}_{i}^{\top}}=\frac{\partial\left[\boldsymbol{\Sigma}_{\omega i}^{1 / 2} \mathbf{R}(\rho) \boldsymbol{\Sigma}_{\omega i}^{1 / 2}\right]}{\partial \boldsymbol{\omega}_{i}^{\top}}=\frac{\partial \boldsymbol{\Sigma}_{\omega i}^{1 / 2}}{\partial \boldsymbol{\omega}_{i}^{\top}} \mathbf{R}(\rho) \boldsymbol{\Sigma}_{\omega i}^{1 / 2}+\boldsymbol{\Sigma}_{\omega}^{1 / 2} \mathbf{R} \frac{\partial \boldsymbol{\Sigma}_{\omega}^{1 / 2}}{\partial \boldsymbol{\omega}_{i}^{\top}}
$$

Temos que $\boldsymbol{\Sigma}_{\omega i}=\operatorname{diag}\left\{\mathrm{E}\left(u_{\omega i 1}^{2}\right), \ldots, \mathrm{E}\left(u_{\omega i s_{i}}^{2}\right)\right\}$. Observe que,

$$
\mathrm{E}\left(u_{\omega i j}^{2}\right)=\frac{1}{4} \mathrm{E}\left\{\tanh ^{2}\left(\frac{y_{i j}-\mu_{\omega i j}}{2}\right)\right\}+\frac{1}{4}+\frac{1}{\alpha^{2}}
$$

$\log 0$

$$
\frac{\partial \mathrm{E}\left(u_{i j}^{2}\right)}{\partial \mu_{\omega i j}} \frac{\partial y_{\omega i j}}{\partial \omega_{i j}}=\frac{\partial}{\partial \mu_{\omega i j}} \int_{-\infty}^{\infty}\left\{\frac{1}{4} \tanh ^{2}\left(\frac{y_{i j}-\mu_{\omega i j}}{2}\right) f\left(y_{i j}, \mu_{\omega i j}\right)\right\} d y_{i j} \times \frac{\partial \mu_{\omega i j}}{\partial \omega_{i j}} .
$$

Através de manipulação similar ao que foi feito na Seção B.1 chegamos em,

$$
\frac{\partial \mathrm{E}\left(u_{i j}^{2}\right)}{\partial \mu_{\omega i j}}=0
$$

e assim,

$$
\frac{\partial \mathbf{C}}{\partial \boldsymbol{\omega}^{\top}}=\operatorname{diag}\left\{\frac{\partial \mathbf{C}_{1}}{\partial \boldsymbol{\omega}_{1}^{\top}}, \ldots, \frac{\partial \mathbf{C}_{n}}{\partial \boldsymbol{\omega}_{n}^{\top}}\right\}=\mathbf{0}
$$

Agora derivamos $\mathbf{u}_{\omega}$ com relação à $\boldsymbol{\omega}^{\top}$. A forma matricial das derivadas de $\mathbf{u}$ com relação à $\omega$ é dada por $\frac{\partial \mathbf{u}_{\omega}}{\partial \boldsymbol{\omega}^{\top}}=\dot{\mathcal{U}}_{x} \operatorname{com} \dot{\mathcal{U}}_{x}=\operatorname{diag}\left\{\dot{\mathcal{U}}_{x 1}, \ldots, \dot{\mathcal{U}}_{x n}\right\}, 0$ em que $\dot{\mathcal{U}}_{x i}=\operatorname{diag}\left\{\frac{\partial u_{\omega i 1}}{\partial \omega_{i 1}}, \ldots, \frac{\partial u_{\omega i s}}{\partial \omega_{i s}}\right\}$. Temos que,

$$
u_{\omega i j}=-\frac{1}{2} \operatorname{tgh}\left(\frac{y_{i j}-\mu_{\omega i j}}{2}\right)+\frac{1}{\alpha^{2}} \operatorname{senh}\left(y_{i j}-\mu_{\omega i j}\right) .
$$

de forma que

$$
\frac{\partial u_{\omega i j}}{\partial \omega_{i j}}=\left\{\frac{1}{4} \operatorname{sech}^{2}\left(\frac{y_{i j}-\mu_{\omega i j}}{2}\right)-\frac{1}{\alpha^{2}}\left[1+2 \operatorname{senh}^{2}\left(\frac{y_{i j}-\mu_{\omega i j}}{2}\right)\right]\right\} s_{x k} .
$$


Por fim, derivamos $\mathbf{N}_{\omega i}$ com relação a $\boldsymbol{\omega}_{i}^{\top}$.

$$
\mathbf{N}_{\omega i}=\mathrm{E}\left(\frac{\partial \mathbf{u}_{\omega i}}{\partial \boldsymbol{\mu}_{\omega i}}\right)=\operatorname{diag}\left\{\mathrm{E}\left(\dot{u}_{\omega i 1}\right), \ldots, \mathrm{E}\left(\dot{u}_{\omega i s}\right)\right\},
$$

em que,

$$
\mathrm{E}\left(\dot{u}_{\omega i j}\right)=\frac{1}{4} \mathrm{E}\left\{\operatorname{sech}^{2}\left(\frac{y_{i j}-\mu_{\omega i j}}{2}\right)\right\}-\frac{1}{2}-\frac{1}{\alpha^{2}} .
$$

$\mathrm{e}$

$$
\begin{aligned}
\frac{\partial \mathrm{E}_{\mu_{i j}}\left\{\dot{u}_{i j}\left(y_{i j}, \mu_{i j}\right)\right\}}{\partial \mu_{i j}}= & \int_{-\infty}^{\infty}\left\{\frac{1}{\alpha \sqrt{2 \pi}} \operatorname{senh}\left(\frac{y_{i j}-\mu_{i j}}{2}\right) \exp \left\{-2 \alpha^{-2} \operatorname{senh}^{2}\left(\frac{y_{i j}-\mu_{i j}}{2}\right)\right\}\right. \\
& \left.\times\left[\frac{1}{2 \alpha^{2}}+\frac{1}{8} \operatorname{sech}^{2}\left(\frac{y_{i j}-\mu_{i j}}{2}\right)\right]\right\} d y_{i j} \\
= & 0 .
\end{aligned}
$$

Portanto,

$$
\boldsymbol{\Delta}=\frac{\partial \boldsymbol{\Psi}(\boldsymbol{\mu} \mid \boldsymbol{\omega})}{\partial \boldsymbol{\omega}^{\top}}=\mathbf{X}_{\omega}^{\top} \mathbf{N}_{\omega} \mathbf{C}_{\omega}^{-1} \frac{\partial \mathbf{u}_{\omega}}{\partial \boldsymbol{\omega}^{\top}}+\frac{\partial \mathbf{X}_{\omega}^{\top}}{\partial \boldsymbol{\omega}^{\top}} \mathbf{N}_{\omega} \mathbf{C}_{\omega}^{-1} \mathbf{u}_{\omega}
$$


Apêndice C

Tabelas referentes às simulações do

Capítulo 2 
Tabela C.1: Estimativas médias de $\beta_{0}, \beta_{1}, \alpha$ e $\rho$, viés relativo (VR) (em \%) e erro quadrático médio (EQM) de $\hat{\beta}_{0}$ e $\hat{\beta}_{1}$ do estudo de simulação dos quais os dados são gerados de uma distribuição multivariada BS com uma estrutura de correlação AR-1 e ajustado sob o modelo log-BS (via equações de estimação) com mesma estrutura de correlação.

\begin{tabular}{|c|c|c|c|c|c|c|c|c|c|c|c|}
\hline \multirow{4}{*}{$\rho$} & \multirow{3}{*}{$\mathrm{s}$} & \multicolumn{3}{|c|}{$\hat{\beta}_{0}$} & \multirow[b]{2}{*}{$\beta_{1}$} & \multicolumn{2}{|c|}{$\hat{\beta}_{1}$} & \multirow[b]{2}{*}{$\alpha$} & \multicolumn{2}{|c|}{$\alpha$} & \multirow[b]{2}{*}{$\rho$} \\
\hline & & $\beta_{0}$ & VR & EQM & & VR & EQI & & VR & EQM & \\
\hline & & \multicolumn{10}{|c|}{$n=10$} \\
\hline & 3 & 4,0003 & 0,0068 & 0,0394 & $-2,0004$ & 0224 & 0,0820 & 0,4744 & $-5,1218$ & 0,0050 & 0,2290 \\
\hline \multirow[t]{3}{*}{0,3} & 5 & 4,0000 & 0,0011 & 0,0183 & $-1,9996$ & $-0,0219$ & 0,0480 & 0,4835 & $-3,2964$ & 0,0032 & 0,2582 \\
\hline & 10 & 4,0005 & 0,0132 & 0,0101 & $-1,9997$ & $-0,0143$ & 0,0241 & 0,4914 & $-1,7166$ & 0,0015 & 0,2771 \\
\hline & 3 & 4,0049 & 0,1224 & 0,0294 & $-2,0078$ & 0,3910 & 0,0622 & 0,4672 & $-6,5674$ & 0,0074 & 0,5242 \\
\hline \multirow[t]{3}{*}{0,6} & 5 & 3,9978 & $-0,0542$ & 0,0190 & $-1,9969$ & $-0,1571$ & 0,0341 & 0,4760 & $-4,8022$ & 0,0049 & 0,5443 \\
\hline & 10 & 4,0012 & 0,0304 & 0,0108 & $-2,0029$ & 0,1434 & 0,0138 & 0,4861 & $-2,7712$ & 0,0024 & 0,5655 \\
\hline & 3 & 4,0024 & 0,0594 & 0,0278 & $-1,9976$ & $-0,1190$ & 0,0265 & 0,4512 & $-9,7611$ & 0,0120 & 0,8126 \\
\hline \multirow[t]{4}{*}{0,9} & 5 & 4,0004 & 0,0093 & 0,0206 & $-2,0014$ & 0,0679 & 0,0064 & 0,4632 & $-7,3664$ & 0,0095 & 0,8475 \\
\hline & 10 & 4,0045 & 0,1118 & 0,0160 & $-2,0004$ & 0,0214 & 0,0028 & 0,4 & $-5,4184$ & 0,0072 & 0,8703 \\
\hline & & \multicolumn{10}{|c|}{$n=20$} \\
\hline & 3 & 3,9973 & $-0,0671$ & 0,0147 & $-1,9980$ & $-0,1017$ & 0,0419 & 0,4879 & $-2,4112$ & 0,0024 & 0,2695 \\
\hline \multirow[t]{3}{*}{0,3} & 5 & 3,9977 & $-0,0587$ & 0,0101 & $-1,9979$ & $-0,1064$ & 0,0265 & 0,4922 & $-1,5547$ & 0,0015 & 0,2770 \\
\hline & 10 & 4,0007 & 0,0172 & 0,0050 & $-2,0013$ & 0,0650 & 0,0113 & & $-0,8222$ & 0,0008 & 0,2874 \\
\hline & 3 & 4,0000 & 0,0012 & 0,0154 & $-2,0$ & 0,1683 & 0,0 & 30 & $-3,3924$ & 0,0034 & 0,5588 \\
\hline \multirow[t]{3}{*}{0,6} & 5 & 4,0001 & 0,0019 & 0,0093 & $-1,9998$ & $-0,0090$ & 0,0155 & 0,4889 & $-2,2271$ & 0,0023 & 0,5708 \\
\hline & 10 & 3,9998 & $-0,0041$ & 0,0055 & $-2,0000$ & 0,0017 & 0,0075 & 0,4928 & $-1,4332$ & 0,0013 & 0,5810 \\
\hline & 3 & 3,9985 & $-0,0380$ & 0,0121 & $-2,0005$ & 0,0270 & 0,0079 & 0,4770 & $-4,5921$ & 0,0056 & 0,8676 \\
\hline \multirow[t]{4}{*}{0,9} & 5 & 4,0000 & 0,0008 & 0,0104 & $-1,9995$ & $-0,0250$ & 0,0034 & 0,4818 & $-3,6451$ & 0,0048 & 0,8754 \\
\hline & 10 & 4,0012 & 0,0299 & 0,0079 & $-2,0000$ & $-0,0009$ & 0,0016 & 0,4858 & $-2,8459$ & 0,0035 & 0,8858 \\
\hline & & \multicolumn{10}{|c|}{$n=50$} \\
\hline & 3 & 3,9988 & $-0,0303$ & 0,0071 & $-1,9981$ & $-0,0975$ & 0,0198 & 0,4949 & $-1,0259$ & 0,0010 & 0,2844 \\
\hline \multirow[t]{3}{*}{0,3} & 5 & 4,0003 & 0,0080 & 0,0040 & $-2,0018$ & 0,0907 & 0,0102 & 0,4970 & $-0,6005$ & 0,0006 & 0,2892 \\
\hline & 10 & 4,0000 & 0,0007 & 0,0020 & $-2,0007$ & 0,0356 & 0,0046 & 0,4981 & $-0,3804$ & 0,0003 & 0,2939 \\
\hline & 3 & 4,0021 & 0,0515 & 0,0055 & $-2,0018$ & 0,0878 & 0,0099 & 0,4938 & $-1,2315$ & 0,0013 & 0,5834 \\
\hline \multirow[t]{3}{*}{0,6} & 5 & 4,0010 & 0,0251 & 0,0038 & $-2,0026$ & 0,1302 & 0,0060 & 952 & $-0,9637$ & 0,0009 & 0,5861 \\
\hline & 10 & 993 & $-0,0167$ & 0,0020 & -1 , & $-0,0083$ & & & $-0,5480$ & 0,0005 & 0,5922 \\
\hline & 3 & 3,9997 & $-0,0067$ & 0,0047 & $-2,0004$ & 0,0204 & 0,0028 & 0,4915 & $-1,6998$ & 0,0021 & 0,8893 \\
\hline \multirow[t]{4}{*}{0,9} & 5 & 3,9981 & $-0,0473$ & 0,0042 & $-1,9999$ & $-0,0072$ & 0,0012 & 0,4922 & $-1,5645$ & 0,0019 & 0,8899 \\
\hline & 10 & 4,0010 & 0,0253 & 0,0034 & $-1,9996$ & $-0,0184$ & 0,0006 & 0,4947 & $-1,0653$ & 0,0014 & 0,8938 \\
\hline & & \multicolumn{10}{|c|}{$n=80$} \\
\hline & 3 & 4,000 & 76 & 0,0 & $-2,0$ & 0,0974 & 0,0109 & 0 , & $-0,7315$ & 0,0006 & 0,2911 \\
\hline \multirow[t]{3}{*}{0,3} & 5 & 4,0006 & 0,0155 & 0,0023 & $-2,0005$ & 0,0242 & 0,0 & 0,4 & $-0,3253$ & 0,0004 & 0,2943 \\
\hline & 10 & 4,0001 & 0,0032 & 0,0013 & $-2,0009$ & 0,0431 & 0,0032 & 0,4989 & $-0,2123$ & 0,0002 & 0,2963 \\
\hline & 3 & 3,9987 & $-0,0320$ & 0,0035 & $-1,9988$ & $-0,0577$ & 0,0063 & 0,4958 & $-0,8396$ & 0,0009 & 0,5893 \\
\hline 0,6 & 5 & 4,0001 & 0,0015 & 0,0024 & $-1,9995$ & $-0,0238$ & 0,0038 & 0,4967 & $-0,6688$ & 0,0006 & 0,5904 \\
\hline & 10 & 3,9994 & $-0,0140$ & 0,0013 & $-1,9999$ & $-0,0039$ & 0,0018 & 0,4982 & $-0,3503$ & 0,0003 & 0,5947 \\
\hline & 3 & 3,9995 & $-0,0122$ & 0,0032 & $-1,9990$ & $-0,0510$ & 0,0019 & 0,4942 & $-1,1542$ & 0,0014 & 0,8913 \\
\hline 0,9 & 5 & 4,0022 & 0,0546 & 0,0027 & $-2,0007$ & 0,0348 & 0,0010 & 0,4948 & $-1,0337$ & 0,0012 & 0,8939 \\
\hline & 10 & 3,9997 & $-0,0081$ & 0,0022 & $-2,0003$ & 0,0162 & 0,0004 & 0,4965 & $-0,6925$ & 0,0009 & 0,8955 \\
\hline
\end{tabular}


Tabela C.2: Estimativas médias de $\beta_{0}, \beta_{1}, \alpha$ e $\rho$, viés relativo (VR) (em \%) e erro quadrático médio (EQM) de $\hat{\beta}_{0}$ e $\hat{\beta}_{1}$ do estudo de simulação dos quais os dados são gerados de uma distribuição multivariada BS com uma estrutura de correlação permutável e ajustado sob o modelo log-BS (via equações de estimação) com mesma estrutura de correlação.

\begin{tabular}{|c|c|c|c|c|c|c|c|c|c|c|c|}
\hline \multirow{4}{*}{$\rho$} & \multirow{3}{*}{$\mathrm{s}$} & \multicolumn{3}{|c|}{$\hat{\beta}_{0}$} & \multirow[b]{2}{*}{$\beta_{1}$} & \multicolumn{2}{|c|}{$\hat{\beta}_{1}$} & \multirow[b]{2}{*}{$\alpha$} & \multicolumn{2}{|c|}{$u$} & \multirow[b]{2}{*}{$\rho$} \\
\hline & & $\beta_{0}$ & VR & EQM & & VR & EQM & & VR & EQM & \\
\hline & & \multicolumn{10}{|c|}{$n=10$} \\
\hline & 3 & 4,0025 & 0,0627 & 0,0331 & $-2,0039$ & 0,1966 & 0,0746 & 0,4713 & $-5,7315$ & 0,0056 & 0,2305 \\
\hline \multirow{3}{*}{0,3} & 5 & 4,0008 & 0,0209 & 0,0242 & $-2,0017$ & 0,0868 & 0,0492 & 0,4800 & $-4,0021$ & 0,0036 & 0,2471 \\
\hline & 10 & 4,0000 & 0,0000 & 0,0132 & $-2,0009$ & 0,0473 & 0,0178 & 0,4856 & $-2,8826$ & 0,0024 & 0,2544 \\
\hline & 3 & 4,0059 & 0,1466 & 0,0260 & $-2,0037$ & 0,1846 & 0,0501 & 0,4644 & $-7,1272$ & 0,0077 & 0,5000 \\
\hline \multirow[t]{3}{*}{0,6} & 5 & 4,0031 & 0,0771 & 0,0220 & $-2,0027$ & 0,1347 & 0,0275 & 0,4717 & $-5,6506$ & 0,0061 & 0,5245 \\
\hline & 10 & 4,0017 & 0,0433 & 0,0190 & $-2,0009$ & 0,0437 & 0,0161 & 0,4738 & $-5,2350$ & 0,0055 & 0,5236 \\
\hline & 3 & 3,9990 & $-0,0261$ & 0,0260 & $-2,0000$ & 0,0013 & 0,0145 & 0,4546 & $-9,0780$ & 0,0120 & 0,8319 \\
\hline \multirow[t]{4}{*}{0,9} & 5 & 4,0037 & 0,0920 & 0,0238 & $-2,0006$ & 0,0292 & 0,0073 & 0,4612 & $-7,7502$ & 0,0114 & 0,8506 \\
\hline & 10 & 3,9990 & $-0,0245$ & 0,0224 & $-2,0006$ & 0,0301 & 0,0029 & 0,4603 & $-7,9490$ & 0,0114 & 0,8586 \\
\hline & & \multicolumn{10}{|c|}{$n=20$} \\
\hline & 3 & 4,0004 & 0,0088 & 0,0167 & $-1,9990$ & $-0,0513$ & 0,0398 & 0,4870 & $-2,5983$ & 0,0025 & 0,2638 \\
\hline \multirow[t]{3}{*}{0,3} & 5 & 4,0004 & 0,0112 & 0,0106 & $-2,0006$ & 0,0295 & 0,0231 & 0,4900 & $-2,0018$ & 0,0018 & 0,2712 \\
\hline & 10 & 4,0013 & 0,0331 & 0,0069 & $-2,0035$ & 0,1750 & 0,0100 & 0,4931 & $-1,3896$ & 0,0012 & 0,2781 \\
\hline & 3 & 3,9990 & $-0,0255$ & 0,0196 & $-2,0029$ & 0,1456 & 0,0385 & 0,4 & $-3,8393$ & 0,0038 & 0,5440 \\
\hline \multirow[t]{3}{*}{0,6} & 5 & 3,9981 & $-0,0483$ & 0,0116 & $-2,0017$ & 0,0828 & 0,0113 & 0,4873 & $-2,5377$ & 0,0031 & 0,5634 \\
\hline & 10 & 4,0009 & 0,0232 & 0,0093 & $-1,9994$ & $-0,0295$ & 0,0061 & 0,4877 & $-2,4613$ & 0,0026 & 0,5644 \\
\hline & 3 & 3,9985 & $-0,0378$ & 0,0131 & $-1,9985$ & $-0,0772$ & 0,0090 & 0,4749 & $-5,0174$ & 0,0059 & 0,8634 \\
\hline \multirow[t]{4}{*}{0,9} & 5 & 4,0009 & 0,0236 & 0,0119 & $-2,0000$ & $-0,0020$ & 0,0033 & 0,4810 & $-3,7923$ & 0,0055 & 0,8759 \\
\hline & 10 & 4,0030 & 0,0743 & 0,0112 & $-2,0013$ & 0,0649 & 0,0019 & 0,4815 & $-3,7079$ & 0,0054 & 0,8753 \\
\hline & & \multicolumn{10}{|c|}{$n=50$} \\
\hline & 3 & 4,0009 & 0,0218 & 0,0067 & $-2,0010$ & 0,0522 & 0,0158 & 0,4937 & $-1,2501$ & 0,0010 & 0,2826 \\
\hline \multirow[t]{3}{*}{0,3} & 5 & 4,0013 & 0,0322 & 0,0044 & $-2,0026$ & 0,1287 & 0,0095 & 0,4966 & $-0,6886$ & 0,0007 & 0,2869 \\
\hline & 10 & 4,0004 & 0,0111 & 0,0028 & $-2,0004$ & 0,0182 & 0,0046 & 0,4966 & $-0,6712$ & 0,0004 & 0,2883 \\
\hline & 3 & 4,0006 & 0,0160 & 0,0059 & $-2,0005$ & 0,0274 & 0,0104 & 0,4944 & $-1,1260$ & 0,0015 & 0,5804 \\
\hline \multirow[t]{3}{*}{0,6} & 5 & 4,0008 & 0,0212 & 0,0045 & $-1,9998$ & $-0,0096$ & 0,0055 & 0,4942 & $-1,1507$ & 0,0012 & 0,5833 \\
\hline & 10 & 3,9996 & $-0,0108$ & 0,0036 & $-1,4$ & $-0,0058$ & 0,0025 & 0 & $-1,0579$ & 0,0011 & 0,5844 \\
\hline & 3 & 3,9992 & $-0,0191$ & 0,0051 & $-2,0008$ & 0,0402 & 0,0030 & 0,4915 & $-1,7048$ & 0,0022 & 0,8864 \\
\hline \multirow[t]{4}{*}{0,9} & 5 & 3,9996 & $-0,0105$ & 0,0047 & $-2,0007$ & 0,0344 & 0,0014 & 0,4916 & $-1,6796$ & 0,0021 & 0,8891 \\
\hline & 10 & 3,9987 & $-0,0328$ & 0,0043 & $-2,0004$ & 0,0212 & 0,0006 & 0,4928 & $-1,4382$ & 0,0021 & 0,8918 \\
\hline & & \multicolumn{10}{|c|}{$n=80$} \\
\hline & 3 & 4,0001 & 0,0025 & 0,0041 & $-1,9994$ & $-0,0300$ & 0,0106 & 0,4962 & $-0,7674$ & 0,0006 & 0,2909 \\
\hline \multirow[t]{3}{*}{0,3} & 5 & 3,9999 & $-0,0026$ & 0,0027 & $-1,9988$ & $-0,0610$ & 0,0057 & 0,4978 & $-0,4425$ & 0,0004 & 0,2927 \\
\hline & 10 & 3,9997 & $-0,0083$ & 0,0017 & $-2,0005$ & 0,0258 & 0,0027 & 0,4979 & $-0,4148$ & 0,0003 & 0,2920 \\
\hline & 3 & 3,9992 & $-0,0207$ & 0,0038 & $-1,9986$ & $-0,0709$ & 0,0060 & 0,4956 & $-0,8872$ & 0,0009 & 0,5875 \\
\hline 0,6 & 5 & 3,9990 & $-0,0248$ & 0,0029 & $-2,0001$ & 0,0053 & 0,0035 & 0,4964 & $-0,7213$ & 0,0008 & 0,5881 \\
\hline & 10 & 3,9999 & $-0,0014$ & 0,0022 & $-1,9995$ & $-0,0251$ & 0,0016 & 0,4967 & $-0,6557$ & 0,0007 & 0,5891 \\
\hline & 3 & 4,0000 & 0,0000 & 0,0031 & $-1,9991$ & $-0,0445$ & 0,0016 & 0,4948 & $-1,0410$ & 0,0014 & 0,8918 \\
\hline 0,9 & 5 & 3,9996 & $-0,0089$ & 0,0031 & $-2,0002$ & 0,0099 & 0,0010 & 0,4949 & $-1,0195$ & 0,0013 & 0,8929 \\
\hline & 10 & 3,9994 & $-0,0144$ & 0,0027 & $-2,0003$ & 0,0161 & 0,0004 & 0,4955 & $-0,8907$ & 0,0013 & 0,8943 \\
\hline
\end{tabular}


Tabela C.3: Estimativas médias de $\beta_{0}, \beta_{1}, \alpha$ e $\rho$, viés relativo (VR) (em \%) e erro quadrático médio (EQM) de $\hat{\beta}_{0}$ e $\hat{\beta}_{1}$ do estudo de simulação dos quais os dados são gerados de uma distribuição multivariada $B S$ com uma estrutura de correlação AR-1 e ajustado sob o modelo log-BS (via equações de estimação) com estrutura de correlação permutável.

\begin{tabular}{|c|c|c|c|c|c|c|c|c|c|c|c|}
\hline \multirow{4}{*}{$\rho$} & \multirow{3}{*}{$\mathrm{s}$} & \multicolumn{3}{|c|}{$\hat{\beta}_{0}$} & \multirow[b]{2}{*}{$\beta_{1}$} & \multicolumn{2}{|c|}{$\hat{\beta}_{1}$} & \multirow[b]{2}{*}{$\alpha$} & \multicolumn{2}{|c|}{$\alpha$} & \multirow[b]{2}{*}{$\rho$} \\
\hline & & $\beta_{0}$ & VR & EQM & & VR & EQM & & VR & EQM & \\
\hline & & \multicolumn{10}{|c|}{$n=10$} \\
\hline & 3 & 3,9972 & $-0,0709$ & 0,0537 & $-1,9$ & $-0,1373$ & 0,1263 & 0,4726 & $-5,4754$ & 0,0050 & 0,1681 \\
\hline \multirow[t]{3}{*}{0,3} & 5 & 4,0014 & 0,0350 & 0,0188 & $-2,0015$ & 0,0745 & 0,0457 & 0,4845 & $-3,0910$ & 0,0030 & 0,1190 \\
\hline & 10 & 3,9985 & $-0,0368$ & 0,0095 & $-2,0004$ & 0,0182 & 0,0227 & 0,4913 & $-1,7331$ & 0,0015 & 0,0611 \\
\hline & 3 & 3,9972 & $-0,0694$ & 0,0281 & $-1,9950$ & $-0,2516$ & 0,0700 & 0,4669 & $-6,6173$ & 0,0070 & 0,4247 \\
\hline \multirow[t]{3}{*}{0,6} & 5 & 3,9992 & $-0,0209$ & 0,0240 & $-1,9955$ & $-0,2248$ & 0,0466 & 0,4755 & $-4,8975$ & 0,0046 & 0,3426 \\
\hline & 10 & 4,0008 & 0,0203 & 0,0130 & $-1,9994$ & $-0,0307$ & 0,0215 & 0,4862 & $-2,7557$ & 0,0025 & 0,2112 \\
\hline & 3 & 4,0005 & 0,0124 & 0,0232 & $-1,9991$ & $-0,0473$ & 0,0148 & 0,4614 & $-7,7293$ & 0,0114 & 0,8217 \\
\hline \multirow[t]{4}{*}{0,9} & 5 & 3,9993 & $-0,0166$ & 0,0234 & $-1,9976$ & $-0,1176$ & 0,0165 & 0,4619 & $-7,6102$ & 0,0100 & 0,7405 \\
\hline & 10 & 4,0022 & 0,0538 & 0,0198 & $-2,0011$ & 0,0539 & 0,0082 & 0,4696 & $-6,0767$ & 0,0073 & 0,6297 \\
\hline & & \multicolumn{10}{|c|}{$n=20$} \\
\hline & 3 & 3,9999 & $-0,0027$ & 0,0167 & $-2,0003$ & 0,0148 & 0,0386 & 0,4869 & $-2,6128$ & 0,0024 & 0,1965 \\
\hline \multirow[t]{3}{*}{0,3} & 5 & 3,9992 & $-0,0204$ & 0,0103 & $-1,9985$ & $-0,0759$ & 0,0249 & 0,4915 & $-1,6999$ & 0,0015 & 0,1351 \\
\hline & 10 & 3,9999 & $-0,0027$ & 0,0057 & $-1,9990$ & $-0,0485$ & 0,0150 & & $-0,7544$ & 0,0007 & 0,0714 \\
\hline & 3 & 4,0013 & 0,0319 & 0,0166 & $-2,0$ & 0,1138 & 0,0402 & 28 & $-3,4384$ & 0,0035 & 0,4681 \\
\hline \multirow[t]{3}{*}{0,6} & 5 & 3,9989 & $-0,0278$ & 0,0105 & $-1,9957$ & $-0,2144$ & 0,0191 & 0,4875 & $-2,4955$ & 0,0023 & 0,3679 \\
\hline & 10 & 4,0005 & 0,0114 & 0,0067 & $-2,0014$ & 0,0690 & 0,0127 & 0,4934 & $-1,3242$ & 0,0012 & 0,2289 \\
\hline & 3 & 4,0006 & 0,0138 & 0,0127 & $-2,0019$ & 0,0968 & 0,0073 & 0,4792 & $-4,1631$ & 0,0054 & 0,8388 \\
\hline \multirow[t]{4}{*}{0,9} & 5 & 4,0006 & 0,0140 & 0,0112 & $-2,0027$ & 0,1328 & 0,0073 & 0,4821 & $-3,5849$ & 0,0049 & 0,7812 \\
\hline & 10 & 4,0013 & 0,0322 & 0,0099 & $-2,0001$ & 0,0062 & 0,0055 & 0,4844 & $-3,1241$ & 0,0036 & 0,6615 \\
\hline & & \multicolumn{10}{|c|}{$n=50$} \\
\hline & 3 & 3,9996 & $-0,0097$ & 0,0068 & $-1,9991$ & $-0,0468$ & 0,0160 & 0,4948 & $-1,0391$ & 0,0010 & 0,2159 \\
\hline \multirow[t]{3}{*}{0,3} & 5 & 4,0006 & 0,0155 & 0,0040 & $-2,0013$ & 0,0644 & 0,0099 & 0,4966 & $-0,6797$ & 0,0006 & 0,1453 \\
\hline & 10 & 4,0007 & 0,0171 & 0,0021 & $-2,0010$ & 0,0498 & 0,0052 & 0,4984 & $-0,3180$ & 0,0003 & 0,0781 \\
\hline & 3 & 3,9993 & $-0,0169$ & 0,0060 & $-1,9995$ & $-0,0249$ & 0,0116 & 0,4926 & $-1,4779$ & 0,0014 & 0,5009 \\
\hline \multirow[t]{3}{*}{0,6} & 5 & 3,9991 & $-0,0237$ & 0,0044 & $-1,9991$ & $-0,0451$ & 0,0075 & 954 & $-0,9122$ & 0,0009 & 0,3903 \\
\hline & 10 & 4,0002 & & 0,0024 & & $-0,0013$ & 0,0038 & & $-0,5849$ & 0,0005 & 132 \\
\hline & 3 & 4,0013 & 0,0315 & 0,0052 & $-2,0007$ & 0,0328 & 0,0044 & 0,4912 & $-1,7514$ & 0,0021 & 0,8558 \\
\hline \multirow[t]{4}{*}{0,9} & 5 & 4,0004 & 0,0091 & 0,0049 & $-2,0008$ & 0,0420 & 0,0028 & 0,4927 & $-1,4613$ & 0,0019 & 0,8004 \\
\hline & 10 & 4,0002 & 0,0062 & 0,0038 & $-2,0010$ & 0,0490 & 0,0013 & 0,4943 & $-1,1414$ & 0,0014 & 0,6840 \\
\hline & & \multicolumn{10}{|c|}{$n=80$} \\
\hline & 3 & 3,9991 & 217 & 0,0037 & $-1,9$ & $-0,0390$ & 0,0116 & 68 & $-0,6351$ & 0,0006 & 0,2201 \\
\hline \multirow[t]{3}{*}{0,3} & 5 & 4,0000 & $-0,0011$ & 0,0028 & $-2,0000$ & 0,0003 & 0,0069 & & $-0,4385$ & 0,0004 & 0,1471 \\
\hline & 10 & 3,9997 & $-0,0073$ & 0,0013 & $-1,9998$ & $-0,0098$ & 0,0035 & 0,4989 & $-0,2261$ & 0,0002 & 0,0789 \\
\hline & 3 & 3,9992 & $-0,0200$ & 0,0042 & $-1,9993$ & $-0,0352$ & 0,0080 & 0,4962 & $-0,7563$ & 0,0008 & 0,5077 \\
\hline 0,6 & 5 & 3,9989 & $-0,0283$ & 0,0029 & $-1,9993$ & $-0,0342$ & 0,0049 & 0,4974 & $-0,5237$ & 0,0005 & 0,3949 \\
\hline & 10 & 3,9989 & $-0,0273$ & 0,0017 & $-2,0007$ & 0,0325 & 0,0027 & 0,4985 & $-0,2926$ & 0,0003 & 0,2455 \\
\hline & 3 & 3,9993 & $-0,0180$ & 0,0032 & $-2,0002$ & 0,0119 & 0,0022 & 0,4944 & $-1,1181$ & 0,0013 & 0,8608 \\
\hline 0,9 & 5 & 3,9999 & $-0,0034$ & 0,0029 & $-2,0006$ & 0,0313 & 0,0017 & 0,4949 & $-1,0263$ & 0,0012 & 0,8051 \\
\hline & 10 & 3,9995 & $-0,0137$ & 0,0025 & $-1,9985$ & $-0,0734$ & 0,0012 & 0,4959 & $-0,8211$ & 0,0009 & 0,6874 \\
\hline
\end{tabular}




\section{Apêndice D}

\section{Cálculos do Capítulo 3}

\section{D.1 Função de estimação para o parâmetro de localização}

Iremos apresentar nesta seção os cálculos detalhados para a obtenção da função de estimação ótima descrita em (3.5). Temos que

$$
u_{i j}=-\xi_{i j 2}\left(\frac{1}{2 \xi_{i j 1}}-2 w_{i j} \xi_{i j 1}\right),
$$

em que em que $w_{i j}=\frac{\nu+1}{\nu+4 \xi_{i j 2}^{2}}$.

Observe que,

$$
\begin{aligned}
\dot{u}_{i j}= & \frac{\partial u_{i j}}{\partial \mu_{i j}}=-\frac{\partial \xi_{i j 2}}{\partial \mu_{i j}}\left(\frac{1}{2 \xi_{i j 1}}-\frac{2(\nu+1) \xi_{i j 1}}{\nu+4 \xi_{i j 2}^{2}}\right)-\xi_{i j 2}\left\{\frac{1}{2}\left(-\frac{1}{\xi_{i j 1}^{2}}\right)\left(\frac{\partial \xi_{i j 1}}{\partial \mu_{i j}}\right)\right. \\
& \left.-\frac{2(\nu+1)}{\left(\nu+4 \xi_{i j 2}^{2}\right)^{2}}\left[\left(\frac{\partial \xi_{i j 1}}{\partial \mu_{i j}}\right)\left(\nu+4 \xi_{i j 2}^{2}\right)-\xi_{i j 1}\left(8 \xi_{i j 2} \frac{\partial \xi_{i j 2}}{\partial \mu_{i j}}\right)\right]\right\} \\
= & \frac{\xi_{i j 1}}{2}\left(\frac{1}{2 \xi_{i j 1}}-\frac{2(\nu+1) \xi_{i j 1}}{\nu+4 \xi_{i j 2}^{2}}\right)-\xi_{i j 2}\left\{\frac{1}{2}\left(-\frac{1}{\xi_{i j 1}^{2}}\right)\left(\frac{-\xi_{i j 2}}{2}\right)\right. \\
& \left.-\frac{2(\nu+1)}{\left(\nu+4 \xi_{i j 2}^{2}\right)^{2}}\left[\left(\frac{-\xi_{i j 2}}{2}\right)\left(\nu+4 \xi_{i j 2}^{2}\right)-\xi_{i j 1}\left(8 \xi_{i j 2} \frac{-\xi_{i j 1}}{2}\right)\right]\right\} \\
= & \frac{1}{4}-\frac{(\nu+1) \xi_{i j 1}^{2}}{\nu+4 \xi_{i j 2}^{2}}-\frac{\xi_{i j 2}^{2}}{4 \xi_{i j 1}^{2}}+\frac{2(\nu+1) \xi_{i j 2}}{\left(\nu+4 \xi_{i j 2}^{2}\right)^{2}}\left[\left(\frac{-\xi_{i j 2}}{2}\right)\left(\nu+4 \xi_{i j 2}^{2}\right)+\frac{8 \xi_{i j 2} \xi_{i j 1}^{2}}{2}\right] \\
= & \frac{1}{4}-\frac{(\nu+1) \xi_{i j 1}^{2}}{\nu+4 \xi_{i j 2}^{2}}-\frac{\xi_{i j 2}^{2}}{4 \xi_{i j 1}^{2}}-\frac{(\nu+1) \xi_{i j 2}^{2}}{\left(\nu+4 \xi_{i j 2}^{2}\right)}+\frac{(\nu+1) 8 \xi_{i j 2}^{2} \xi_{i j 1}^{2}}{\left(\nu+4 \xi_{i j 2}^{2}\right)^{2}} \\
= & \frac{1}{4}\left(1-\frac{\xi_{i j 2}^{2}}{\xi_{i j 1}^{2}}\right)-\frac{(\nu+1)}{\nu+4 \xi_{i j 2}^{2}}\left(\xi_{i j 1}^{2}+\xi_{i j 2}^{2}\right)+\frac{(\nu+1) 8 \xi_{i j 2}^{2} \xi_{i j 1}^{2}}{\left(\nu+4 \xi_{i j 2}^{2}\right)^{2}} \\
= & \frac{1}{4 \alpha^{2} \xi_{i j 1}^{2}}-\frac{(\nu+1)}{\nu+4 \xi_{i j 2}^{2}}\left(\xi_{i j 1}^{2}+\xi_{i j 2}^{2}\right)+\frac{(\nu+1) 8 \xi_{i j 2}^{2} \xi_{i j 1}^{2}}{\left(\nu+4 \xi_{i j 2}^{2}\right)^{2}} \\
= & \frac{1}{4 \alpha^{2} \xi_{i j 1}^{2}}-w_{i j}\left(\xi_{i j 1}^{2}+\xi_{i j 2}^{2}\right)+\frac{w_{i j} 8 \xi_{i j 2}^{2} \xi_{i j 1}^{2}}{\left(\nu+4 \xi_{i j 2}^{2}\right)} .
\end{aligned}
$$


Reescrevemos,

$$
\begin{aligned}
\dot{u}_{i j}= & \frac{\partial u_{i j}}{\partial \gamma_{i j}}=\frac{1}{4 \alpha^{2}\left(\frac{1}{\alpha^{2}}+\xi_{i j 2}^{2}\right)}-\frac{(\nu+1)}{\nu+4 \xi_{i j 2}^{2}}\left(\left(\frac{1}{\alpha^{2}}+\xi_{i j 2}^{2}\right)+\xi_{i j 2}^{2}\right) \\
& +\frac{(\nu+1) 8 \xi_{i j 2}^{2}\left(\frac{1}{\alpha^{2}}+\xi_{i j 2}^{2}\right)}{\left(\nu+4 \xi_{i j 2}^{2}\right)^{2}} \\
= & \frac{1}{4+4 \xi_{i j 2}^{2} \alpha^{2}}-\frac{(\nu+1)}{\nu+4 \xi_{i j 2}^{2}}\left(\frac{1}{\alpha^{2}}+2 \xi_{i j 2}^{2}\right)+\frac{(\nu+1) 8 \xi_{i j 2}^{2}\left(\frac{1}{\alpha^{2}}+\xi_{i j 2}^{2}\right)}{\left(\nu+4 \xi_{i j 2}^{2}\right)^{2}} \\
= & \frac{1}{4+4 \xi_{i j 2}^{2} \alpha^{2}}-w_{i j}\left(\frac{1}{\alpha^{2}}+2 \xi_{i j 2}^{2}\right)+8 \xi_{i j 2}^{2} w_{i j}\left(\frac{1}{\alpha^{2}}+\xi_{i j 2}^{2}\right) \frac{1}{\left(\nu+4 \xi_{i j 2}^{2}\right)} .
\end{aligned}
$$

Realizando uma mudança de variável e tomando $x_{i j}=\xi_{i j 2} \alpha$ temos que

$$
\begin{aligned}
\mathrm{E}\left(\dot{u}_{i j}\right)=\int_{-\infty}^{\infty} & \left\{\frac { 2 } { \alpha } \mathrm { c } ( \nu ) \{ 1 + \frac { 4 } { \nu } ( \frac { x _ { i j } } { \alpha } ) ^ { 2 } \} ^ { - ( \frac { \nu + 1 } { 2 } ) } \left[\frac{1}{4+4 x_{i j}^{2}}-w_{i j}\left(\frac{1+2 x_{i j}^{2}}{\alpha^{2}}\right)\right.\right. \\
& \left.\left.+8 \frac{x_{i j}^{2} w_{i j}}{\left(\alpha^{2} \nu+4 x i_{i j}^{2}\right)}\left(\frac{1+x_{i j}^{2}}{\alpha^{2}}\right)\right]\right\} \mathrm{d} x_{i j},
\end{aligned}
$$

em que passamos a escrever $\left.w_{i j}=(1+\nu) \alpha^{2} /\left(\alpha^{2} \nu+4 x i_{i j}^{2}\right)\right)$. Observe que reescrever $\dot{u}_{i j}$ permite que a integral dada em (D.1) se torne mais simples para implementar computacionalmente uma vez que não é em função de funções hipoerbólicas.

Denotamos,

$$
\mathbf{N}_{i}=\mathrm{E}\left(\frac{\partial \mathbf{u}_{i}}{\partial \boldsymbol{\mu}_{i}}\right)=\operatorname{diag}\left\{\mathrm{E}\left(\dot{u}_{i 1}\right), \ldots, \mathrm{E}\left(\dot{u}_{i s}\right)\right\}
$$

então,

$$
\mathrm{E}\left(\frac{\partial \mathbf{u}_{i}}{\partial \boldsymbol{\beta}^{\top}}\right)^{\top}=\mathrm{E}\left(\frac{\partial \mathbf{u}_{i}}{\partial \boldsymbol{\mu}_{i}} \frac{\partial \boldsymbol{\mu}_{i}}{\partial \boldsymbol{\eta}_{i}} \frac{\partial \boldsymbol{\eta}_{i}}{\partial \boldsymbol{\beta}^{\top}}\right)^{\top}=\mathbf{X}_{i}^{\top} \mathbf{N}_{i}
$$

A matriz de variância-covariâncias de $\mathbf{u}_{i}$ pode ser escrita na forma

$$
\operatorname{Cov}\left(\mathbf{u}_{i}\right)=\operatorname{Var}\left(\mathbf{u}_{i}\right)^{1 / 2} \operatorname{Corr}\left(\mathbf{u}_{i}\right) \operatorname{Var}\left(\mathbf{u}_{i}\right)^{1 / 2},
$$

em que $\operatorname{Corr}\left(\mathbf{u}_{i}\right)$ denota a matriz de correlação de $\mathbf{u}_{i}$ e $\operatorname{Var}\left(\mathbf{u}_{i}\right)$ é dada por,

$$
\operatorname{Var}\left(\mathbf{u}_{i}\right)=\operatorname{diag}\left\{\operatorname{Var}\left(u_{i 1}\right), \ldots, \operatorname{Var}\left(u_{i s}\right)\right\}=\operatorname{diag}\left\{\mathrm{E}\left(u_{i 1}^{2}\right), \ldots, \mathrm{E}\left(u_{i s}^{2}\right)\right\}=\boldsymbol{\Sigma}_{i}
$$

em que,

$$
\begin{aligned}
u_{i j}^{2} & =\left[-\xi_{i j 2}\left(\frac{1}{2 \xi_{i j 1}}-2 w_{i j} \xi_{i j 1}\right)\right]^{2} \\
& =\xi_{i j 2}^{2}\left[\frac{1}{4 \xi_{i j 1}^{2}}-2 w_{i j}+4 w_{i j}^{2} \xi_{i j 1}^{2}\right]
\end{aligned}
$$




$$
=\xi_{i j 2}^{2}\left[\frac{1}{4\left(\frac{1}{\alpha^{2}}+\xi_{i j 2}^{2}\right)}-2 w_{i j}+4 w_{i j}^{2}\left(\frac{1}{\alpha^{2}}+\xi_{i j 2}^{2}\right)\right] .
$$

Realizando uma mudança de variável e tomando $x_{i j}=\xi_{i j 2} \alpha$ temos que

$$
\begin{aligned}
\operatorname{Var}\left(u_{i j}\right)=\mathrm{E}\left(u_{i j}^{2}\right)=\int_{-\infty}^{\infty} & \left\{\frac{2}{\alpha} \mathrm{c}(\nu)\left\{1+\frac{4}{\nu}\left(\frac{x_{i j}}{\alpha}\right)^{2}\right\}^{-\left(\frac{\nu+1}{2}\right)} \frac{x_{i j}^{2}}{\alpha^{2}}\right. \\
& {\left.\left[\frac{\alpha^{2}}{4\left(1+x_{i j}^{2}\right)}-2 w_{i j}+4 w_{i j}^{2}\left(\frac{1+x_{i j}^{2}}{\alpha^{2}}\right)\right]\right\} \mathrm{d} x_{i j} . }
\end{aligned}
$$

Logo,

$$
\boldsymbol{\Psi}_{n}^{*}(\boldsymbol{\beta})=\sum_{i=1}^{n} \mathbf{X}_{i}^{\top} \mathbf{N}_{i}\left(\boldsymbol{\Sigma}_{i}^{1 / 2} \mathbf{R}\left(\mathbf{u}_{i}\right) \boldsymbol{\Sigma}_{i}^{1 / 2}\right)^{-1} \mathbf{u}_{i} .
$$

\section{D.2 Matrizes de sensibilidade e variabilidade}

Iremos derivar nesta seção a matriz de sensibilidade, $\mathbf{S}_{\mathbf{i}}(\boldsymbol{\beta})$, e a matriz de variabilidade, $\mathbf{V}_{\mathbf{i}}(\boldsymbol{\beta})$, para a função de estimação (3.5).

Observe inicialmente que,

$$
\frac{\partial \boldsymbol{\psi}_{i}(\boldsymbol{\beta})}{\partial \boldsymbol{\beta}}=\frac{\partial \boldsymbol{\psi}_{i}(\boldsymbol{\beta})}{\partial \boldsymbol{\mu}_{i}} \times \frac{\partial \boldsymbol{\mu}_{i}}{\partial \boldsymbol{\eta}_{i}} \times \frac{\partial \boldsymbol{\eta}_{i}}{\partial \boldsymbol{\beta}}
$$

Temos que,

$$
\begin{aligned}
\frac{\partial \boldsymbol{\psi}_{i}(\boldsymbol{\beta})}{\partial \boldsymbol{\mu}_{i}}= & \frac{\partial}{\partial \boldsymbol{\mu}_{i}}\left\{\mathbf{X}_{i}^{\top} \mathbf{N}_{i} \boldsymbol{\Sigma}_{i}^{-1 / 2} \mathbf{R}(\boldsymbol{\rho})^{-1} \boldsymbol{\Sigma}_{i}^{-1 / 2} \mathbf{u}_{i}\right\} \\
= & \mathbf{X}_{i}^{\top} \frac{\partial \mathbf{N}_{i}}{\partial \boldsymbol{\mu}_{i}} \boldsymbol{\Sigma}_{i}^{-1 / 2} \mathbf{R}(\boldsymbol{\rho})^{-1} \boldsymbol{\Sigma}_{i}^{-1 / 2} \mathbf{u}_{i}+\mathbf{X}_{i}^{\top} \mathbf{N}_{i} \frac{\partial \boldsymbol{\Sigma}_{i}^{-1 / 2}}{\partial \boldsymbol{\mu}_{i}} \mathbf{R}(\boldsymbol{\rho})^{-1} \boldsymbol{\Sigma}_{i}^{-1 / 2} \mathbf{u}_{i} \\
& +\mathbf{X}_{i}^{\top} \mathbf{N}_{i} \boldsymbol{\Sigma}_{i}^{-1 / 2} \mathbf{R}(\boldsymbol{\rho})^{-1} \frac{\partial \boldsymbol{\Sigma}_{i}^{-1 / 2}}{\partial \boldsymbol{\mu}_{i}} \mathbf{u}_{i}+\mathbf{X}_{i}^{\top} \mathbf{N}_{i} \boldsymbol{\Sigma}_{i}^{-1 / 2} \mathbf{R}(\boldsymbol{\rho})^{-1} \boldsymbol{\Sigma}_{i}^{-1 / 2} \frac{\partial \mathbf{u}_{i}}{\partial \boldsymbol{\mu}_{i}}
\end{aligned}
$$

Note que

$$
\begin{aligned}
\mathrm{E}\left(\dot{u}_{i j}\right)=\int_{-\infty}^{\infty} & \left\{\mathrm { c } ( \nu ) \xi _ { i j 1 } \{ 1 + \frac { 4 \xi _ { i j 2 } ^ { 2 } } { \nu } \} ^ { - ( \frac { \nu + 1 } { 2 } ) } \left[\frac{1}{4 \alpha^{2} \xi_{i j 1}^{2}}-w_{i j}\left(\xi_{i j 1}^{2}+\xi_{i j 2}^{2}\right)\right.\right. \\
& \left.\left.+\frac{w_{i j} 8 \xi_{i j 2}^{2} \xi_{i j 1}^{2}}{\left(\nu+4 \xi_{i j 2}^{2}\right)}\right]\right\} \mathrm{d} x_{i j} .
\end{aligned}
$$

Temos que,

$$
\frac{d \xi_{i j 1}}{d \mu_{i j}}=\frac{-1}{2} \xi_{i j 2}, \frac{d \xi_{i j 2}}{d \mu_{i j}}=\frac{-1}{2} \xi_{i j 1}
$$


$\mathrm{e}$

$$
\frac{d w_{i j}}{d \mu_{i j}}=\frac{-(\nu+1)\left(-4 \xi_{i j 2} \xi_{i j 1}\right)}{\left(\nu+4 \xi_{i j 2}^{2}\right)^{2}}=\frac{4 w \xi_{i j 2} \xi_{i j 1}}{\nu+4 \xi_{i j 2}^{2}} .
$$

Então

$$
\begin{aligned}
& \frac{d}{d \mu_{i j}}\left\{\mathrm{c}(\nu) \xi_{i j 1}\left[1+\frac{4 \xi_{i j 2}^{2}}{\nu}\right]^{-\left(\frac{\nu+1}{2}\right)}\left[\frac{1}{4 \alpha^{2} \xi_{i j 1}^{2}}-w_{i j}\left(\xi_{i j 1}^{2}+\xi_{i j 2}^{2}\right)+\frac{w_{i j} 8 \xi_{i j 2}^{2} \xi_{i j 1}^{2}}{\left(\nu+4 \xi_{i j 2}^{2}\right)}\right]\right\} \\
& =\mathrm{c}(\nu)\left\{-\frac{\xi_{i j 2}}{2}\left\{1+\frac{4 \xi_{i j 2}^{2}}{\nu}\right\}^{-\left(\frac{\nu+1}{2}\right)}\left[\frac{1}{4 \alpha^{2} \xi_{i j 1}^{2}}-w_{i j}\left(\xi_{i j 1}^{2}+\xi_{i j 2}^{2}\right)+\frac{w_{i j} 8 \xi_{i j 2}^{2} \xi_{i j 1}^{2}}{\left(\nu+4 \xi_{i j 2}^{2}\right)}\right]\right. \\
& -\left(\frac{\nu+1}{2}\right) \xi_{i j 1}\left[1+\frac{4 \xi_{i j 2}^{2}}{\nu}\right]^{-\left(\frac{\nu+3}{2}\right)}\left[-\frac{4 \xi_{i j 2} \xi_{i j 1}}{\nu}\right]\left[\frac{1}{4 \alpha^{2} \xi_{i j 1}^{2}}-w_{i j}\left(\xi_{i j 1}^{2}+\xi_{i j 2}^{2}\right)\right. \\
& +\frac{w_{i j} 8 \xi_{i j 2}^{2} \xi_{i j 1}^{2}}{\left(\nu+4 \xi_{i j 2}^{2}\right)}\left[+\xi_{i j 1}\left[1+\frac{4 \xi_{i j 2}^{2}}{\nu}\right]^{-\left(\frac{\nu+1}{2}\right)}\left[-\frac{4 \alpha^{2} \xi_{i j 1} \xi_{i j 2}}{\left(4 \alpha^{2} \xi_{i j 1}^{2}\right)^{2}}-\frac{4 w \xi_{i j 2} \xi_{i j 1}}{\nu+4 \xi_{i j 2}^{2}}\left(\xi_{i j 1}^{2}+\xi_{i j 2}^{2}\right)\right.\right. \\
& \left.\left.+2 w_{i j} \xi_{i j 1} \xi_{i j 2}+\frac{32 w_{i j} \xi_{i j 2}^{3} \xi_{i j 1}^{3}-\left(\nu+4 \xi_{i j 2}^{2}\right) 8 w_{i j} \xi_{i j 2} \xi_{i j 1}\left(\xi_{i j 2}^{2}+\xi_{i j 1}^{2}\right)+4 \xi_{i j 2} \xi_{i j 1}\left(w_{i j} 8 \xi_{i j 2}^{2} \xi_{i j 1}^{2}\right)}{\left(\nu+4 \xi_{i j 2}^{2}\right)^{2}}\right]\right\} .
\end{aligned}
$$

Podemos provar facilmente que $\left\{\mathrm{c}(\nu) \xi_{i j 1}\left\{1+\frac{4 \xi_{i j 2}^{2}}{\nu}\right\}^{-\left(\frac{\nu+1}{2}\right)}\left[\frac{1}{4 \alpha^{2} \xi_{i j 1}^{2}}-w_{i j}\left(\xi_{i j 1}^{2}+\xi_{i j 2}^{2}\right)+\frac{w_{i j} 8 \xi_{i j 2}^{2} \xi_{i j 1}^{2}}{\left(\nu+4 \xi_{i j 2}^{2}\right)}\right]\right\}$ é contínua. Logo, podemos derivar sob o sinal de integração e fazendo as modificações de variáveis adequadas temos que,

$$
\begin{aligned}
& \frac{\partial \mathrm{E}\left\{\dot{u}_{i j}\left(y_{i j}, \mu_{i j}\right)\right\}}{\partial \mu_{i j}}=\int_{-\infty}^{\infty}\left\{\mathrm { c } ( \nu ) \left\{-\frac{\xi_{i j 2}}{2}\left\{1+\frac{4 \xi_{i j 2}^{2}}{\nu}\right\}^{-\left(\frac{\nu+1}{2}\right)}\left[\frac{1}{4 \alpha^{2} \xi_{i j 1}^{2}}\right.\right.\right. \\
& \left.-w_{i j}\left(\xi_{i j 1}^{2}+\xi_{i j 2}^{2}\right)+\frac{w_{i j} 8 \xi_{i j 2}^{2} \xi_{i j 1}^{2}}{\left(\nu+4 \xi_{i j 2}^{2}\right)}\right]-\xi_{i j 1}\left(\frac{\nu+1}{2}\right)\left[1+\frac{4 \xi_{i j 2}^{2}}{\nu}\right]^{-\left(\frac{\nu+3}{2}\right)} \\
& {\left[-\frac{4 \xi_{i j 2} \xi_{i j 1}}{\nu}\right]\left[\frac{1}{4 \alpha^{2} \xi_{i j 1}^{2}}-w_{i j}\left(\xi_{i j 1}^{2}+\xi_{i j 2}^{2}\right)+\frac{w_{i j} 8 \xi_{i j 2}^{2} \xi_{i j 1}^{2}}{\left(\nu+4 \xi_{i j 2}^{2}\right)}\right]} \\
& +\xi_{i j 1}\left[1+\frac{4 \xi_{i j 2}^{2}}{\nu}\right]^{-\left(\frac{\nu+1}{2}\right)}\left[\frac{4 \alpha^{2} \xi_{i j 1} \xi_{i j 2}}{\left(4 \alpha^{2} \xi_{i j 1}^{2}\right)^{2}}-\frac{4 w \xi_{i j 2} \xi_{i j 1}}{\nu+4 \xi_{i j 2}^{2}}\left(\xi_{i j 1}^{2}+\xi_{i j 2}^{2}\right)\right. \\
& +2 w_{i j} \xi_{i j 1} \xi_{i j 2}+\frac{32 w_{i j} \xi_{i j 2}^{3} \xi_{i j 1}^{3}}{\left(\nu+4 \xi_{i j 2}^{2}\right)^{2}} \\
& \left.\left.\left.\frac{-\left(\nu+4 \xi_{i j 2}^{2}\right) 8 w_{i j} \xi_{i j 2} \xi_{i j 1}\left(\xi_{i j 2}^{2}+\xi_{i j 1}^{2}\right)+4 \xi_{i j 2} \xi_{i j 1}\left(w_{i j} 8 \xi_{i j 2}^{2} \xi_{i j 1}^{2}\right)}{\left(\nu+4 \xi_{i j 2}^{2}\right)^{2}}\right]\right\}\right\} d y_{i j} \\
& =0 \text {. }
\end{aligned}
$$

Portanto,

$$
\frac{\partial \mathbf{N}_{i}}{\partial \boldsymbol{\mu}_{i}}=\mathbf{0}
$$


E ainda temos

$$
\begin{aligned}
\mathrm{E}\left(u_{i j}^{2}\right)=\int_{-\infty}^{\infty} & \left\{\mathrm{c}(\nu) \xi_{i j 1}\left\{1+\frac{4 \xi_{i j 2}^{2}}{\nu}\right\}^{-\left(\frac{\nu+1}{2}\right)} \xi_{i j 2}^{2}\right. \\
& {\left.\left[\frac{\alpha^{2}}{4\left(1+\xi_{i j 2}^{2} \alpha^{2}\right)}-2 w_{i j}+4 w_{i j}^{2}\left(\frac{1+\xi_{i j 2}^{2} \alpha^{2}}{\alpha^{2}}\right)\right]^{2}\right\} \mathrm{d} x_{i j} . }
\end{aligned}
$$

Analogamente, temos que

$$
\left\{\mathrm { c } ( \nu ) \xi _ { i j 1 } \{ 1 + \frac { 4 \xi _ { i j 2 } ^ { 2 } } { \nu } \} ^ { - ( \frac { \nu + 1 } { 2 } ) } \xi _ { i j 2 } ^ { 2 } \left[\frac{\alpha^{2}}{4\left(1+\xi_{i j 2}^{2} \alpha^{2}\right)}\right.\right.
$$

$\left.\left.-2 w_{i j}+4 w_{i j}^{2}\left(\frac{1+\xi_{i j 2}^{2} \alpha^{2}}{\alpha^{2}}\right)\right]^{2}\right\}$ é contínua. Logo, podemos derivar sob o sinal de integração. A derivada do integrando fica dada por

$$
\begin{aligned}
& \frac{d}{d \mu_{i j}}\left\{\mathrm{c}(\nu) \xi_{i j 1}\left\{1+\frac{4 \xi_{i j 2}^{2}}{\nu}\right\}^{-\left(\frac{\nu+1}{2}\right)} \xi_{i j 2}^{2}\left[\frac{\alpha^{2}}{4\left(1+\xi_{i j 2}^{2} \alpha^{2}\right)}-2 w_{i j}+4 w_{i j}^{2}\left(\frac{1+\xi_{i j 2}^{2} \alpha^{2}}{\alpha^{2}}\right)\right]\right\} \\
& =\mathrm{c}(\nu)\left\{-\frac{\xi_{i j 2}}{2}\left\{1+\frac{4 \xi_{i j 2}^{2}}{\nu}\right\}^{-\left(\frac{\nu+1}{2}\right)} \xi_{i j 2}^{2}\left[\frac{\alpha^{2}}{4\left(1+\xi_{i j 2}^{2} \alpha^{2}\right)}-2 w_{i j}+4 w_{i j}^{2}\left(\frac{1+\xi_{i j 2}^{2} \alpha^{2}}{\alpha^{2}}\right)\right]\right. \\
& -\left(\frac{\nu+1}{2}\right) \xi_{i j 1}\left[1+\frac{4 \xi_{i j 2}^{2}}{\nu}\right]^{-\left(\frac{\nu+3}{2}\right)}\left[-\frac{4 \xi_{i j 2} \xi_{i j 1}}{\nu}\right] \xi_{i j 2}^{2}\left[\frac{\alpha^{2}}{4\left(1+\xi_{i j 2}^{2} \alpha^{2}\right)}-2 w_{i j}\right. \\
& \left.+4 w_{i j}^{2}\left(\frac{1+\xi_{i j 2}^{2} \alpha^{2}}{\alpha^{2}}\right)\right]-\mathrm{c}(\nu) \xi_{i j 1}\left\{1+\frac{4 \xi_{i j 2}^{2}}{\nu}\right\}^{-\left(\frac{\nu+1}{2}\right)} \xi_{i j 2} \xi_{i j 1}\left[\frac{\alpha^{2}}{4\left(1+\xi_{i j 2}^{2} \alpha^{2}\right)}-2 w_{i j}\right. \\
& \left.+4 w_{i j}^{2}\left(\frac{1+\xi_{i j 2}^{2} \alpha^{2}}{\alpha^{2}}\right)\right]+\mathrm{c}(\nu) \xi_{i j 1}\left\{1+\frac{4 \xi_{i j 2}^{2}}{\nu}\right\}^{-\left(\frac{\nu+1}{2}\right)}\left[\xi _ { i j 2 } ^ { 2 } \left[\frac{4 \alpha^{2} \xi_{i j 1} \xi_{i j 2}}{16\left(1+\xi_{i j 2}^{2} \alpha^{2}\right)^{2}}\right.\right. \\
& \left.\left.-2 \frac{4 w_{i j} \xi_{i j 2} \xi_{i j 1}}{\nu+4 \xi_{i j 2}^{2}}+8 w_{i j} \frac{4 w_{i j} \xi_{i j 2} \xi_{i j 1}}{\nu+4 \xi_{i j 2}^{2}}\left(\frac{1+\xi_{i j 2}^{2} \alpha^{2}}{\alpha^{2}}\right)-4 w_{i j}^{2} \xi_{i j 2} \xi_{i j 1}\right]\right\}
\end{aligned}
$$

Portanto, realizando algumas mudanças de variáveis podemos ver que

$$
\frac{\partial \mathrm{E}_{\mu_{i j}}\left\{u_{i j}^{2}\left(y_{i j}, \mu_{i j}\right)\right\}}{\partial \mu_{i j}}=0 .
$$

E ainda temos,

$$
\frac{\partial}{\partial \mu_{i j}}\left\{\mathrm{E}\left(u_{i j}^{2}\right)^{-1 / 2}\right\}=-\frac{1}{2} \frac{1}{\mathrm{E}\left(u_{i j}^{2}\right)^{3 / 2}} \frac{\partial \mathrm{E}\left\{u_{i j}^{2}\left(y_{i j}, \mu_{i j}\right)\right\}}{\partial \mu_{i j}}=0
$$


de onde segue que,

$$
\frac{\partial \boldsymbol{\Sigma}_{i}^{-1 / 2}}{\partial \boldsymbol{\mu}_{i}}=\mathbf{0}
$$

Portanto, aplicando (F.2) e (D.5) em (D.3) obtemos

$$
\begin{aligned}
\frac{\partial \boldsymbol{\psi}_{i}(\boldsymbol{\beta})}{\partial \boldsymbol{\mu}_{i}} & =\mathbf{X}_{i}^{\top} \mathbf{N}_{i} \boldsymbol{\Sigma}_{i}^{-1 / 2} \mathbf{R}(\boldsymbol{\rho})^{-1} \boldsymbol{\Sigma}_{i}^{-1 / 2} \frac{\partial \mathbf{u}_{i}}{\partial \boldsymbol{\mu}_{i}} \\
& =\mathbf{X}_{i}^{\top} \mathbf{N}_{i} \boldsymbol{\Sigma}_{i}^{-1 / 2} \mathbf{R}(\boldsymbol{\rho})^{-1} \boldsymbol{\Sigma}_{i}^{-1 / 2} \dot{\mathbf{U}}_{i}
\end{aligned}
$$

em que $\dot{\mathbf{U}}_{i}=\operatorname{diag}\left\{\dot{u}_{i j}, \ldots, \dot{u}_{i s}\right\}$. Logo,

$$
\mathbf{S}_{\mathbf{i}}(\boldsymbol{\beta})=\mathrm{E}\left(\frac{\partial \boldsymbol{\psi}_{i}(\boldsymbol{\beta})}{\partial \boldsymbol{\beta}}\right)=\mathbf{X}_{i}^{\top} \mathbf{N}_{i} \boldsymbol{\Sigma}_{i}^{-1 / 2} \mathbf{R}(\boldsymbol{\rho})^{-1} \boldsymbol{\Sigma}_{i}^{-1 / 2} \mathbf{N}_{i} \mathbf{X}_{i}
$$

Temos ainda que,

$$
\begin{aligned}
\mathbf{V}_{i}(\boldsymbol{\beta})=\mathrm{E}\left[\boldsymbol{\psi}_{i}(\boldsymbol{\beta}) \boldsymbol{\psi}_{i}^{T}(\boldsymbol{\beta})\right] & =\mathrm{E}\left[\mathbf{X}_{i}^{\top} \mathbf{N}_{i} \mathbf{W}_{i}^{-1} \mathbf{u}_{i} \mathbf{u}_{i}^{\top} \mathbf{W}_{i}^{-\mathrm{T}} \mathbf{N}_{i} \mathbf{X}_{i}\right] \\
& =\mathbf{X}_{i}^{\top} \mathbf{N}_{i} \mathbf{W}_{i}^{-1} \mathrm{E}\left[\mathbf{u}_{i} \mathbf{u}_{i}^{\top}\right] \mathbf{W}_{i}^{-\mathrm{T}} \mathbf{N}_{i} \mathbf{X}_{i} \\
& =\mathbf{X}_{i}^{\top} \mathbf{N}_{i} \mathbf{W}_{i}^{-1} \operatorname{Cov}\left(\mathbf{u}_{i}\right) \mathbf{W}_{i}^{-\mathrm{T}} \mathbf{N}_{i} \mathbf{X}_{i}
\end{aligned}
$$

\section{D.3 Influência conformal sob homogeneidade do parâmetro de forma}

\section{D.3.1 Perturbação individual das covariáveis}

Nesta seção temos as contas detalhadas das derivadas de $\mathbf{N}_{\omega i}, \mathbf{C}_{\omega i}$ e $\mathbf{u}_{\omega i}$ com relação à $\boldsymbol{\omega}_{i}^{\top}$. Primeiramente, observe que

$$
\frac{\partial \mathbf{C}}{\partial \boldsymbol{\omega}^{\top}}=\operatorname{diag}\left\{\frac{\partial \mathbf{C}_{1}}{\partial \boldsymbol{\omega}_{1}^{\top}}, \ldots, \frac{\partial \mathbf{C}_{n}}{\partial \boldsymbol{\omega}_{n}^{\top}}\right\}
$$

com

$$
\frac{\partial \mathbf{C}_{i}}{\partial \boldsymbol{\omega}_{i}^{\top}}=\frac{\partial\left[\boldsymbol{\Sigma}_{\omega i}^{1 / 2} \mathbf{R}(\rho) \boldsymbol{\Sigma}_{\omega i}^{1 / 2}\right]}{\partial \boldsymbol{\omega}_{i}^{\top}}=\frac{\partial \boldsymbol{\Sigma}_{\omega i}^{1 / 2}}{\partial \boldsymbol{\omega}_{i}^{\top}} \mathbf{R}(\rho) \boldsymbol{\Sigma}_{\omega i}^{1 / 2}+\boldsymbol{\Sigma}_{\omega}^{1 / 2} \mathbf{R} \frac{\partial \boldsymbol{\Sigma}_{\omega}^{1 / 2}}{\partial \boldsymbol{\omega}_{i}^{\top}}
$$

Temos que $\boldsymbol{\Sigma}_{\omega i}=\operatorname{diag}\left\{\mathrm{E}\left(u_{\omega i 1}^{2}\right), \ldots, \mathrm{E}\left(u_{\omega i s_{i}}^{2}\right)\right\},\left(\boldsymbol{\Sigma}_{\omega i}^{1 / 2}=\operatorname{diag}\left\{\operatorname{Var}^{1 / 2}\left(u_{\omega i 1}\right), \ldots, \operatorname{Var}^{1 / 2}\left(u_{\omega i s}\right)\right\}\right)$. E temos,

$$
\frac{\partial \operatorname{Var}^{1 / 2}\left(u_{\omega i j}\right)}{\partial \omega_{i} j}=\frac{1}{2} \operatorname{Var}^{-1 / 2}\left(u_{\omega i j}\right) \frac{\partial \operatorname{Var}\left(u_{\omega i j}\right)}{\partial \omega_{i j}}=\frac{1}{2} \mathrm{E}^{-1 / 2}\left(u_{\omega i j}^{2}\right) \frac{\partial \mathrm{E}\left(u_{\omega i j}^{2}\right)}{\partial \omega_{i j}}
$$

através de manipulação similar ao que foi feito na Seção D.2 chegamos em $\frac{\partial \mathrm{E}\left(u_{i j}^{2}\right)}{\partial \mu_{\omega i j}}=0$ e assim, $\frac{\partial \mathbf{C}_{\omega i}}{\partial \omega_{i}^{\top}}=\mathbf{0}$.

Temos que $\mathbf{N}_{\omega i}=\operatorname{diag}\left\{\mathrm{E}\left(\dot{u}_{\omega i 1}\right), \ldots, \mathrm{E}\left(\dot{u}_{\omega i s}\right)\right\}$, análogo ao que foi feito em D.2 obtemos também que, $\frac{\partial \mathrm{E}\left\{\dot{u}_{i j}\left(y_{i j}, \mu_{i j}\right)\right\}}{\partial \mu_{i j}}=0$. Logo, $\frac{\partial \mathbf{N}_{\omega i}}{\partial \omega_{i}^{\top}}=\mathbf{0}$. 
Agora derivamos $\mathbf{u}_{\omega}$ com relação à $\boldsymbol{\omega}^{\top}$. A forma matricial das derivadas de $\mathbf{u}$ com relação à $\omega$ é dada por $\frac{\partial \mathbf{u}_{\omega}}{\partial \omega^{\top}}=\dot{\mathcal{U}}_{x} \operatorname{com} \dot{\mathcal{U}}_{x}=\operatorname{diag}\left\{\dot{\mathcal{U}}_{x 1}, \ldots, \dot{\mathcal{U}}_{x n}\right\}$, em que $\dot{\mathcal{U}}_{x i}=\operatorname{diag}\left\{\frac{\partial u_{\omega i 1}}{\partial \omega_{i 1}}, \ldots, \frac{\partial u_{\omega i s}}{\partial \omega_{i s}}\right\}$. Temos que,

$$
u_{\omega i j}=-\xi_{\omega i j 2}\left(\frac{1}{2 \xi_{\omega i j 1}}-\frac{2(\nu+1) \xi_{\omega i j 1}}{\nu+4 \xi_{\omega i j 2}^{2}}\right),
$$

em que $\xi_{\omega i j 1}=\frac{1}{\alpha} \cosh \left(\frac{y_{i j}-\mu_{\omega i j}}{2}\right)$ e $\xi_{\omega i j 2}=\frac{1}{\alpha} \operatorname{senh}\left(\frac{y_{i j}-\mu_{\omega i j}}{2}\right)$ e cuja derivada em relação a $\omega_{i j}$ é dada por,

$$
\begin{aligned}
\frac{\partial u_{\omega i j}}{\partial \omega_{i j}}= & \beta_{k} s_{x k}\left[\frac{1}{4+4 \xi_{\omega i j 2}^{2} \alpha^{2}}-\left(\frac{\nu+1}{\nu+4 \xi_{\omega i j 2}^{2}}\right)\left(\frac{1}{\alpha^{2}}+2 \xi_{\omega i j 2}^{2}\right)+8 \xi_{\omega i j 2}^{2}\left(\frac{\nu+1}{\nu+4 \xi_{\omega i j 2}^{2}}\right)\right. \\
& \left.\left(\frac{1}{\alpha^{2}}+\xi_{\omega i j 2}^{2}\right) \frac{1}{\left(\nu+4 \xi_{\omega i j 2}^{2}\right)}\right] .
\end{aligned}
$$


Apêndice E

Tabelas referentes às simulações do

Capítulo 3 
Tabela E.1: Estimativas médias de $\beta_{0}, \beta_{1}, \alpha$ e $\rho$, viés relativo (VR) (em \%) e erro quadrático médio (EQM) de $\hat{\beta}_{0}$ e $\hat{\beta}_{1}$ do estudo de simulação dos quais os dados são gerados considerando uma estrutura de correlação $A R$-1 e ajustado sob o modelo log-BS-t via EEG com mesma estrutura de correlação $(\nu=4)$.

\begin{tabular}{|c|c|c|c|c|c|c|c|c|c|c|c|}
\hline \multirow{4}{*}{$\rho$} & \multirow{3}{*}{$\mathrm{s}$} & \multicolumn{3}{|c|}{$\hat{\beta}_{0}$} & \multirow[b]{2}{*}{$\beta_{1}$} & \multicolumn{2}{|c|}{$\hat{\beta}_{1}$} & \multirow[b]{2}{*}{$\alpha$} & \multicolumn{2}{|c|}{$\alpha$} & \multirow[b]{2}{*}{$\rho$} \\
\hline & & $\beta_{0}$ & VR & EQM & & VR & EQ1 & & VR & EQM & \\
\hline & & \multicolumn{10}{|c|}{$n=10$} \\
\hline & 3 & 4,0147 & 0,3665 & 0,0660 & $-2,0201$ & 1,0036 & 0,1632 & 0,4997 & $-0,0541$ & 0,0741 & 0,2341 \\
\hline \multirow{3}{*}{0,3} & 5 & 3,9949 & $-0,1263$ & 0,0392 & $-1,9810$ & $-0,9491$ & 0,0972 & 0,4960 & $-0,8042$ & 0,0594 & 0,2445 \\
\hline & 10 & 4,0019 & 0,0474 & 0,0206 & $-2,0003$ & 0,0160 & 0,0469 & 0,5092 & 1,8436 & 0,0838 & 0,2702 \\
\hline & 3 & 4,0064 & 0,1599 & 0,0574 & $-2,0059$ & 0,2956 & 0,1289 & 0,4790 & $-4,1905$ & 0,0737 & 0,5241 \\
\hline \multirow[t]{3}{*}{0,6} & 5 & 4,0017 & 0,0427 & 0,0465 & $-2,0157$ & 0,7857 & 0,0827 & 0,4814 & $-3,7298$ & 0,0574 & 0,5330 \\
\hline & 10 & 3,9949 & $-0,1277$ & 0,0200 & $-2,0044$ & 0,2179 & 0,0280 & 0,5063 & 1,2628 & 0,1119 & 0,5474 \\
\hline & 3 & 3,9984 & $-0,0401$ & 0,0458 & $-1,9961$ & $-0,1955$ & 0,0265 & 0,4799 & $-4,0233$ & 0,0776 & 0,8545 \\
\hline \multirow[t]{4}{*}{0,9} & 5 & 3,9990 & $-0,0257$ & 0,0365 & $-2,0027$ & 0,1340 & 0,0160 & 0,4796 & $-4,0840$ & 0,0624 & 0,8630 \\
\hline & 10 & 4,0078 & 0,1947 & 0,0313 & $-2,0034$ & 0,1689 & 0,0067 & & $-0,5255$ & 0,0655 & 54 \\
\hline & & \multicolumn{10}{|c|}{$n=20$} \\
\hline & 3 & 3,9968 & $-0,0803$ & 0,0280 & $-1,9989$ & $-0,0568$ & 0,0656 & 0,5077 & 1,5406 & 0,0628 & 0,2642 \\
\hline \multirow[t]{3}{*}{0,3} & 5 & 4,0007 & 0,0184 & 0,0158 & $-1,9983$ & $-0,0837$ & 0,0401 & 0,4963 & $-0,7482$ & 0,0549 & 0,2597 \\
\hline & 10 & 4,0004 & 0,0111 & 0,0097 & $-1,9992$ & $-0,0423$ & 0,0228 & 0,5352 & 7,0419 & 0,1028 & 0,2721 \\
\hline & 3 & 3,9971 & $-0,0721$ & 0,0291 & $-1,9$ & $-0,3922$ & 0,0537 & 72 & $-2,5683$ & 0,0551 & 0,5510 \\
\hline \multirow[t]{3}{*}{0,6} & 5 & 4,0011 & 0,0284 & 0,0197 & $-1,9942$ & $-0,2915$ & 0,0337 & 0,5 & 2,5406 & 0,0819 & 0,5557 \\
\hline & 10 & 4,0023 & 0,0586 & 0,0109 & $-1,9994$ & $-0,0284$ & 0,0158 & 0,5098 & 1,9567 & 0,0585 & 0,5581 \\
\hline & 3 & 4,0005 & 0,0137 & 0,0233 & $-2,0019$ & 0,0928 & 0,0110 & 0,4931 & $-1,3827$ & 0,0654 & 0,8711 \\
\hline \multirow[t]{4}{*}{0,9} & 5 & 3,9964 & $-0,0911$ & 0,0190 & $-1,9993$ & $-0,0363$ & 0,0071 & 0,4948 & $-1,0369$ & 0,0650 & 0,8717 \\
\hline & 10 & 3,9976 & $-0,0592$ & 0,0162 & $-2,0006$ & 0,0286 & 0,0029 & 0,5202 & 4,0305 & 0,0753 & 0,8724 \\
\hline & & \multicolumn{10}{|c|}{$n=50$} \\
\hline & 3 & 4,00 & 003 & 0,0130 & $-2,0$ & 0,4045 & 0,0364 & & 4,4196 & 0,0752 & 0,2683 \\
\hline \multirow[t]{3}{*}{0,3} & 5 & 4,0063 & 0,1582 & 0,0071 & $-2,0094$ & 0,4706 & 0,0171 & 0,5163 & 3,2514 & 0,0596 & 0,2759 \\
\hline & 10 & 3,9994 & $-0,0143$ & 0,0041 & $-1,9997$ & $-0,0133$ & 0,0095 & 0,5348 & 6,9615 & 0,1076 & 0,2747 \\
\hline & 3 & 4,0028 & 0,0699 & 0,0130 & $-2,0042$ & 0,2087 & 0,0239 & 0,5209 & 4,1726 & 0,0836 & 0,5612 \\
\hline \multirow[t]{3}{*}{0,6} & 5 & 3,9980 & $-0,0488$ & 0,0071 & $-1,9981$ & $-0,0966$ & 0,0131 & 0,5181 & 3,6222 & 0,0901 & 0,5605 \\
\hline & 10 & 3,9991 & $-0,0234$ & 0,0044 & & $-0,0106$ & 0,0 & & 136 & 0,0593 & 0,5644 \\
\hline & 3 & 3,9949 & $-0,1281$ & 0,0094 & -1 , & $-0,1806$ & 0,0051 & 0 & 2,5670 & 0,0679 & 0,8803 \\
\hline \multirow[t]{4}{*}{0,9} & 5 & 3,9971 & $-0,0737$ & 0,0074 & $-1,9974$ & $-0,1312$ & 0,0028 & 0,5079 & 1,5749 & 0,0688 & 0,8812 \\
\hline & 10 & 4,0028 & 0,0699 & 0,0066 & $-1,9998$ & $-0,0081$ & 0,0014 & 0,5145 & 2,8972 & 0,0636 & 0,8807 \\
\hline & & \multicolumn{10}{|c|}{$n=80$} \\
\hline & 3 & $\begin{array}{l}3,9989 \\
\end{array}$ & $-0,0268$ & 0,0079 & $-1,9983$ & $-0,0869$ & 0,0212 & 0,5053 & 1,0639 & 0,0535 & 0,2722 \\
\hline \multirow[t]{3}{*}{0,3} & 5 & 3,9969 & $-0,0777$ & 0,0041 & $-1,9967$ & $-0,1629$ & 0,0118 & 0,5 & 5,2521 & 0,0744 & 0,2770 \\
\hline & 10 & 3,9992 & $-0,0205$ & 0,0024 & $-1,9983$ & $-0,0849$ & & & 3,0942 & 0,0786 & 0,2754 \\
\hline & 3 & 3,9977 & $-0,0579$ & 0,0068 & $-1,9937$ & $-0,3125$ & 0,0134 & 0,5189 & 3,7851 & 0,0752 & 0,5604 \\
\hline 0,6 & 5 & 4,0020 & 0,0496 & 0,0044 & $-2,0063$ & 0,3172 & 0,0072 & 0,5328 & 6,5560 & 0,0928 & 0,5676 \\
\hline & 10 & 4,0005 & 0,0124 & 0,0025 & $-2,0000$ & $-0,0016$ & 0,0033 & 0,5036 & 0,7202 & 0,0658 & 0,5643 \\
\hline & 3 & 3,9978 & $-0,0553$ & 0,0060 & $-1,9974$ & $-0,1288$ & 0,0043 & 0,5242 & 4,8325 & 0,0804 & 0,8806 \\
\hline 0,9 & 5 & 4,0022 & 0,0552 & 0,0049 & $-2,0008$ & 0,0389 & 0,0023 & 0,5193 & 3,8664 & 0,0663 & 0,8811 \\
\hline & 10 & 3,9981 & $-0,0484$ & 0,0039 & $-2,0000$ & $-0,0023$ & 0,0009 & 0,5121 & 2,4251 & 0,0586 & 0,8813 \\
\hline
\end{tabular}


Tabela E.2: Estimativas médias de $\beta_{0}, \beta_{1}, \alpha$ e $\rho$, viés relativo (VR) (em \%) e erro quadrático médio (EQM) de $\hat{\beta}_{0}$ e $\hat{\beta}_{1}$ do estudo de simulação dos quais os dados são gerados de uma distribuição multivariada $B S$ de uma estrutura de correlação EXC e ajustado sob o modelo log-BS-t via GEE com mesma estrutura de correlação $(\nu=4)$.

\begin{tabular}{|c|c|c|c|c|c|c|c|c|c|c|c|}
\hline \multirow{4}{*}{$\rho$} & \multirow{3}{*}{$\mathrm{S}$} & \multirow[b]{2}{*}{$\beta_{0}$} & \multicolumn{2}{|c|}{$\beta_{0}$} & \multirow[b]{2}{*}{$\beta_{1}$} & \multicolumn{2}{|c|}{$\hat{\beta}_{1}$} & \multirow[b]{2}{*}{$\alpha$} & & \multirow[b]{2}{*}{$\rho$} \\
\hline & & & VR & EQM & & VR & EQM & & VR & EQM & \\
\hline & & \multicolumn{10}{|c|}{$n=10$} \\
\hline & 3 & 4,0155 & 0,3867 & 0,0728 & $-2,0209$ & 1,0443 & 0,1656 & 0,4983 & $-0,3356$ & 0,0734 & 0,2312 \\
\hline \multirow{3}{*}{0,3} & 5 & 3,9956 & $-0,1096$ & 0,0430 & $-1,9809$ & $-0,9532$ & 0,0926 & 0,4931 & $-1,3736$ & 0,0595 & 0,2358 \\
\hline & 10 & 4,0030 & 0,0738 & 0,0293 & $-2,0007$ & 0,0354 & 0,0497 & 0,5030 & 0,6063 & 0,0791 & 0,2534 \\
\hline & 3 & 4,0065 & 0,1630 & 0,0621 & $-2,0054$ & 0,2677 & 0,1326 & 0,4770 & $-4,6069$ & 0,0751 & 0,5214 \\
\hline \multirow[t]{3}{*}{0,6} & 5 & 4,0022 & 0,0548 & 0,0525 & $-2,0197$ & 0,9869 & 0,0789 & 0,4799 & $-4,0140$ & 0,0606 & 0,5230 \\
\hline & 10 & 3,9907 & $-0,2315$ & 0,0337 & $-2,0056$ & 0,2794 & 0,0308 & 0,4958 & $-0,8455$ & 0,1177 & 0,5195 \\
\hline & 3 & 3,9972 & $-0,0703$ & 0,0483 & $-1,9939$ & $-0,3073$ & 0,0309 & 0,4786 & $-4,2850$ & 0,0779 & 0,8537 \\
\hline \multirow[t]{4}{*}{0,9} & 5 & 4,0005 & 0,0125 & 0,0423 & $-2,0039$ & 0,1963 & 0,0213 & 0,4766 & $-4,6722$ & 0,0640 & 0,8581 \\
\hline & 10 & 4,0093 & 0,2330 & 0,0416 & $-2,0026$ & 0,1306 & 0,0079 & 0,4887 & $-2,2654$ & 0,0687 & 0,8490 \\
\hline & & $n=20$ & & & & & & & & & \\
\hline & 3 & 3,9965 & $-0,0868$ & 0,0283 & $-1,9983$ & $-0,0846$ & 0,0620 & 0,5076 & 1,5132 & 0,0629 & 0,2646 \\
\hline \multirow[t]{3}{*}{0,3} & 5 & 4,0016 & 0,0411 & 0,0177 & $-2,0003$ & 0,0140 & 0,0378 & 0,4947 & $-1,0590$ & 0,0547 & 0,2533 \\
\hline & 10 & 4,0012 & 0,0290 & 0,0144 & $-1,9991$ & $-0,0449$ & 0,0226 & 0,5331 & 6,6270 & 0,1033 & 0,2649 \\
\hline & 3 & 3,9976 & $-0,0603$ & 0,0336 & $-1,9929$ & $-0,3571$ & 0,0601 & 0,4861 & $-2,7809$ & 0,0552 & 0,5498 \\
\hline \multirow[t]{3}{*}{0,6} & 5 & 4,0013 & 0,0316 & 0,0244 & $-1,9914$ & $-0,4322$ & 0,0347 & 0,5108 & 2,1605 & 0,0834 & 0,5509 \\
\hline & 10 & 4,0035 & 0,0874 & 0,0183 & $-1,9998$ & $-0,0122$ & 0,0172 & 0,5054 & 1,0737 & 0,0602 & 0,5444 \\
\hline & 3 & 4,0012 & 0,0292 & 0,0247 & $-2,0027$ & 0,1346 & 0,0139 & 0,4925 & $-1,5082$ & 0,0656 & 0,8710 \\
\hline \multirow[t]{4}{*}{0,9} & 5 & 3,9954 & $-0,1140$ & 0,0223 & $-1,9984$ & $-0,0797$ & 0,0090 & 0,4940 & $-1,1921$ & 0,0664 & 0,8692 \\
\hline & 10 & 3,9961 & $-0,0978$ & 0,0225 & $-1,9997$ & $-0,0172$ & 0,0039 & 0,5158 & 3,1521 & 0,0769 & 0,8654 \\
\hline & & \multicolumn{10}{|c|}{$n=50$} \\
\hline & 3 & 4,0036 & 0,0889 & 0,0130 & $-2,0072$ & 0,3610 & 0,0359 & 0,5218 & 4,3677 & 0,0755 & 0,2678 \\
\hline \multirow[t]{3}{*}{0,3} & 5 & 4,0066 & 0,1661 & 0,0084 & $-2,0096$ & 0,4799 & 0,0180 & 0,5152 & 3,0382 & 0,0587 & 0,2747 \\
\hline & 10 & 3,9990 & $-0,0251$ & 0,0060 & $-1,9993$ & $-0,0344$ & 0,0095 & 0,5336 & 6,7266 & 0,1073 & 0,2717 \\
\hline & 3 & 4,0024 & 0,0593 & 0,0141 & $-2,0038$ & 0,1881 & 0,0248 & 0,5209 & 4,1728 & 0,0831 & 0,5602 \\
\hline \multirow[t]{3}{*}{0,6} & 5 & 3,9970 & $-0,0761$ & 0,0092 & $-1,9968$ & $-0,1594$ & 0,0150 & 0,5168 & 3,3679 & 0,0924 & 0,5570 \\
\hline & 10 & 3,9980 & $-0,0503$ & 0,0076 & $-1,9$ & $-0,0929$ & 0,0069 & 0,5 & 465 & 0,0586 & 0,5594 \\
\hline & 3 & 3,9956 & $-0,1111$ & 0,0101 & $-1,9979$ & $-0,1073$ & 0,0061 & 0,5127 & 2,5343 & 0,0681 & 0,8804 \\
\hline \multirow[t]{4}{*}{0,9} & 5 & 3,9969 & $-0,0785$ & 0,0086 & $-1,9970$ & $-0,1496$ & 0,0038 & 0,5073 & 1,4662 & 0,0695 & 0,8810 \\
\hline & 10 & 4,0029 & 0,0728 & 0,0092 & $-1,9998$ & $-0,0093$ & 0,0019 & 0,5130 & 2,6017 & 0,0646 & 0,8783 \\
\hline & & \multicolumn{10}{|c|}{$n=80$} \\
\hline & 3 & 3,9989 & $-0,0285$ & 0,0083 & $-1,9978$ & $-0,1075$ & 0,0219 & 0,5052 & 1,0425 & 0,0535 & 0,2723 \\
\hline 0,3 & 5 & 3,9966 & $-0,0859$ & 0,0047 & $-1,9964$ & $-0,1778$ & 0,0120 & 0,5259 & 5,1873 & 0,0745 & 0,2761 \\
\hline & 10 & 3,9991 & $-0,0230$ & 0,0034 & $-1,9982$ & $-0,0877$ & 0,0063 & 0,5144 & 2,8893 & 0,0784 & 0,2722 \\
\hline & 3 & 3,9980 & $-0,0510$ & 0,0075 & $-1,9941$ & $-0,2975$ & 0,0142 & 0,5186 & 3,7227 & 0,0748 & 0,5602 \\
\hline 0,6 & 5 & 4,0018 & 0,0458 & 0,0058 & $-2,0068$ & 0,3402 & 0,0084 & 0,5323 & 6,4571 & 0,0927 & 0,5664 \\
\hline & 10 & 4,0001 & 0,0017 & 0,0044 & $-1,9993$ & $-0,0325$ & 0,0038 & 0,5018 & 0,3554 & 0,0648 & 0,5791 \\
\hline & 3 & 3,9976 & $-0,0598$ & 0,0065 & $-1,9972$ & $-0,1405$ & 0,0051 & 0,5241 & 4,8141 & 0,0803 & 0,8803 \\
\hline 0,9 & 5 & 4,0022 & 0,0550 & 0,0057 & $-2,0009$ & 0,0445 & 0,0028 & 0,5193 & 3,8587 & 0,0672 & 0,8806 \\
\hline & 10 & 3,9981 & $-0,0480$ & 0,0054 & $-2,0000$ & $-0,0006$ & 0,0012 & 0,5117 & 2,3488 & 0,0593 & 0,8798 \\
\hline
\end{tabular}


Tabela E.3: Estimativas médias de $\beta_{0}, \beta_{1}, \alpha$ e $\rho$, viés relativo (VR) (em \%) e erro quadrático médio (EQM) de $\hat{\beta}_{0}$ e $\hat{\beta}_{1}$ do estudo de simulação dos quais os dados são gerados de uma distribuição multivariada $B S$ de uma estrutura de correlação AR1 e ajustado sob o modelo log-BS-t via GEE com mesma estrutura de correlação permutável $(\nu=4)$.

\begin{tabular}{|c|c|c|c|c|c|c|c|c|c|c|c|}
\hline \multirow{3}{*}{$\rho$} & \multirow{3}{*}{$\mathrm{s}$} & \multicolumn{3}{|c|}{$\hat{\beta}_{0}$} & \multirow[b]{2}{*}{$\beta_{1}$} & \multicolumn{2}{|c|}{$\hat{\beta}_{1}$} & \multirow[b]{2}{*}{$\alpha$} & \multicolumn{2}{|c|}{$\alpha$} & \multirow[b]{2}{*}{$\rho$} \\
\hline & & $\beta_{0}$ & VR & EQM & & VR & EQM & & VR & EQM & \\
\hline & & \multicolumn{10}{|c|}{$n=10$} \\
\hline & 3 & 4,0159 & 0,3976 & 0,0605 & $-2,0252$ & 1,2591 & 0,1626 & 0,5107 & ,1469 & 0,049 & 0,1786 \\
\hline \multirow[t]{3}{*}{0,3} & 5 & 3,9979 & $-0,0519$ & 0,0461 & $-1,9992$ & $-0,0398$ & 0,1178 & 0,4933 & 1,3361 & 0,0268 & 0,1074 \\
\hline & 10 & 4,0023 & 0,0581 & 0,0188 & $-2,0049$ & 0,2426 & 0,0397 & 0,5 & 0,0078 & 0,0314 & 0,0514 \\
\hline & 3 & 4,0041 & 0,1037 & 0,0258 & $-2,0304$ & 1,5219 & 0,0747 & 0,4734 & 5,3156 & 0,0298 & 0,4614 \\
\hline \multirow[t]{3}{*}{0,6} & 5 & 4,0293 & 0,7329 & 0,0296 & $-2,0326$ & 1,6324 & 0,057 & 0,4629 & 7,4276 & 0,0217 & 0,3702 \\
\hline & 10 & 4,0021 & 0,0534 & 0,0132 & $-1,9647$ & $-1,7626$ & 0,0257 & 0,4957 & 0,8541 & 0,0262 & 0,2056 \\
\hline & 3 & 3,9943 & $-0,142$ & 0,0487 & $-1,9923$ & $-0,3835$ & 0,0303 & 0,4707 & $-5,864$ & 0,0325 & 0,8311 \\
\hline \multirow[t]{4}{*}{0,9} & 5 & 4,0151 & 0,3772 & 0,0341 & $-1,9918$ & $-0,4119$ & 0,0124 & 0,4852 & $-2,9532$ & 0,0364 & 0,7754 \\
\hline & 10 & 3,9824 & $-0,439$ & 0,0209 & $-1,9976$ & $-0,1202$ & 0,0129 & 0,5018 & 0,3681 & 0,0507 & 0,6779 \\
\hline & & \multicolumn{10}{|c|}{$n=20$} \\
\hline & 3 & 4,0176 & 0,4396 & 0,0247 & $-2,0201$ & 1,0069 & 0,0857 & 0,507 & 1,4079 & 0,0458 & 0,2178 \\
\hline \multirow[t]{3}{*}{0,3} & 5 & 4,0074 & 0,1838 & 0,0138 & $-2,0169$ & 0,8429 & 0,0452 & 0,479 & $-4,1972$ & 0,0255 & 0,134 \\
\hline & 10 & 4,001 & 0,0254 & 0,0069 & $-1,9929$ & $-0,3538$ & 0,025 & 0,4975 & $-0,5081$ & 0,0332 & 0,0758 \\
\hline & 3 & 4,0108 & 0,269 & 0,0268 & $-2,0254$ & 1,2679 & 0,0248 & 0,4747 & $-5,0642$ & 0,0238 & 0,482 \\
\hline \multirow[t]{3}{*}{0,6} & 5 & 4,0181 & 0,4527 & 0,0255 & $-2,0012$ & 0,0608 & 0,0373 & 0,4853 & $-2,9434$ & 0,0241 & 0,3648 \\
\hline & 10 & 3,9776 & $-0,5609$ & 0,0093 & $-1,9951$ & $-0,2466$ & 0,0128 & 0,4968 & $-0,6491$ & 0,0213 & 0,2101 \\
\hline & 3 & 3,9902 & $-0,2461$ & 0,0182 & $-2,0044$ & 0,2197 & 0,0151 & 0,4801 & $-3,9842$ & 0,0218 & 0,852 \\
\hline \multirow[t]{4}{*}{0,9} & 5 & 4,0014 & 0,0342 & 0,0179 & $-1,9997$ & $-0,0165$ & 0,009 & 0,4769 & $-4,6156$ & 0,0255 & 0,7911 \\
\hline & 10 & 3,9947 & $-0,1321$ & 0,0145 & $-2,0048$ & 0,2402 & 0,0072 & 0,4961 & $-0,7775$ & 0,0223 & 0,6718 \\
\hline & & \multicolumn{10}{|c|}{$n=50$} \\
\hline & 3 & 4,0047 & 0,1171 & 0,0088 & $-2,0066$ & 0,3317 & 0,0251 & 0,5112 & 2,2355 & 0,0247 & 0,2024 \\
\hline \multirow[t]{3}{*}{0,3} & 5 & 3,9944 & $-0,1389$ & 0,0059 & $-1,9842$ & $-0,7877$ & 0,0159 & 0,4928 & $-1,4437$ & 0,0205 & 0,1459 \\
\hline & 10 & 4,0119 & 0,2972 & 0,0036 & $-2,0159$ & 0,7963 & 0,0088 & 0,4913 & $-1,7361$ & 0,023 & 0,0722 \\
\hline & 3 & 4,0068 & 0,1689 & 0,0105 & $-1,9867$ & $-0,6632$ & 0,0195 & 0,478 & $-4,2214$ & 0,016 & 0,5112 \\
\hline \multirow{3}{*}{0,6} & 5 & 3,9878 & $-0,3052$ & 0,0065 & $-1,9894$ & $-0,5302$ & 0,0124 & 0,486 & $-2,6645$ & 0,0182 & 0,3665 \\
\hline & 10 & 3,9988 & $-0,0302$ & 0,004 & -1 & $-0,1576$ & 0,0064 & 0,4961 & $-0,7755$ & 0,0285 & 0,2427 \\
\hline & 3 & 3,9812 & $-0,4711$ & 0,0067 & $-1,9856$ & $-0,7198$ & 0,0056 & 0,485 & $-2,988$ & 0,0257 & 0,86 \\
\hline \multirow[t]{4}{*}{0,9} & 5 & 4,0024 & 0,0611 & 0,006 & $-2,0165$ & 0,8271 & 0,0036 & 0,4988 & $-0,2307$ & 0,0268 & 0,7971 \\
\hline & 10 & 4,0089 & 0,2216 & 0,006 & $-2,0092$ & 0,4606 & 0,003 & 0,5219 & 4,381 & 0,0411 & 0,6823 \\
\hline & & \multicolumn{10}{|c|}{$n=$} \\
\hline & 3 & 4,0097 & 0,2437 & 0,0071 & $-2,0069$ & 0,3467 & 0,017 & 0,515 & 3,0019 & 0,0231 & 0,2142 \\
\hline \multirow[t]{3}{*}{0,3} & 5 & 4,0084 & 0,2098 & 0,0036 & $-2,0133$ & 0,6631 & 0,0118 & 0,5214 & 4,2721 & 0,0297 & 0,14 \\
\hline & 10 & 3,9955 & $-0,1122$ & 0,0018 & $-1,9928$ & $-0,358$ & 0,0047 & 0,4969 & $-0,6161$ & 0,0228 & 0,0792 \\
\hline & 3 & 4,0053 & 0,1335 & 0,0056 & $-2,0127$ & 0,6342 & 0,0103 & 0,4845 & $-3,108$ & 0,0211 & 0,4979 \\
\hline 0,6 & 5 & 3,993 & $-0,1748$ & 0,0034 & $-1,9966$ & $-0,1698$ & 0,0063 & 0,4885 & $-2,2993$ & 0,0134 & 0,391 \\
\hline & 10 & 4,0102 & 0,2557 & 0,0022 & $-2,0104$ & 0,5186 & 0,0039 & 0,522 & 4,3905 & 0,0349 & 0,2464 \\
\hline & 3 & 4,0113 & 0,2826 & 0,0045 & $-2,0087$ & 0,4328 & 0,0043 & 0,4767 & $-4,6697$ & 0,018 & 0,8526 \\
\hline 0,9 & 5 & 4,0059 & 0,1468 & 0,0061 & $-2,0006$ & 0,0281 & 0,0041 & 0,5021 & 0,4244 & 0,0293 & 0,801 \\
\hline & 10 & 4,0012 & 0,031 & 0,0029 & $-2,0098$ & 0,4905 & 0,0019 & 0,4884 & $-2,328$ & 0,0316 & 0,6794 \\
\hline
\end{tabular}


Tabela E.4: Estimativas médias de $\beta_{0}, \beta_{1}, \alpha$ e $\rho$, viés relativo (VR) (em \%) e erro quadrático médio (EQM) de $\hat{\beta}_{0}$ e $\hat{\beta}_{1}$ do estudo de simulação dos quais os dados são gerados de uma distribuição multivariada BS de uma estrutura de correlação permutável e ajustado sob o modelo log-BS-t via GEE com mesma estrutura de correlação verdadeira, $(\nu=7)$.

\begin{tabular}{|c|c|c|c|c|c|c|c|c|c|c|c|}
\hline \multirow{4}{*}{$\rho$} & \multirow{3}{*}{$\mathrm{S}$} & \multicolumn{3}{|c|}{$\hat{\beta}_{0}$} & \multirow[b]{2}{*}{$\beta_{1}$} & \multicolumn{2}{|c|}{$\hat{\beta}_{1}$} & \multirow[b]{2}{*}{$\alpha$} & & \multirow[b]{2}{*}{$\rho$} \\
\hline & & $\beta_{0}$ & VR & EQM & & VR & EQM & & VR & EQM & \\
\hline & & \multicolumn{10}{|c|}{$n=10$} \\
\hline & 3 & 4,016 & 0,4005 & 0,0586 & $-2,0249$ & 245 & 0 , & 5113 & 2636 & 0503 & 0,2428 \\
\hline \multirow[t]{3}{*}{0,3} & 5 & 3,9978 & $-0,054$ & 0,0457 & $-2,001$ & 0,0509 & 0,0994 & 0,488 & $-2,4048$ & 0,0266 & 0,2378 \\
\hline & 10 & 4,0015 & 0,0377 & 0,0273 & $-2,0051$ & 0,2572 & 0,0331 & 0,4874 & $-2,5246$ & 0,0307 & 0,2428 \\
\hline & 3 & 4,0042 & 0,1043 & 0,0263 & $-2,0318$ & 1,5916 & 0,0687 & 0,4721 & $-5,5782$ & 0,0306 & 0,5389 \\
\hline \multirow[t]{3}{*}{0,6} & 5 & 4,0273 & 0,6837 & 0,0273 & $-2,0244$ & 1,2225 & 0,0425 & 0,4635 & $-7,2985$ & 0,0241 & 0,56 \\
\hline & 10 & 4,0138 & 0,3447 & 0,0199 & $-1,9746$ & $-1,2684$ & 0,0154 & 0,4858 & $-2,8371$ & 0,0292 & 0,5266 \\
\hline & 3 & 3,9964 & $-0,0904$ & 0,0483 & -1, & $-0,2596$ & 0,0259 & 0,4694 & $-6,1286$ & 0,0329 & 0,8686 \\
\hline \multirow[t]{4}{*}{0,9} & 5 & 4,0148 & 0,3696 & 0,0366 & -1, & $-0,5182$ & 0,0082 & 0,483 & $-3,3967$ & 0,037 & 0,8719 \\
\hline & 10 & 815 & $-0,4633$ & 0,024 & & $-0,1278$ & 0,004 & 0,5027 & 0,5479 & 0,0528 & 8829 \\
\hline & & \multicolumn{10}{|c|}{$n=20$} \\
\hline & 3 & 4,0153 & 0,3829 & 0,024 & $-2,0156$ & 0,7821 & 0,0789 & 0,507 & 1,4048 & 0,0453 & 0,2857 \\
\hline \multirow[t]{3}{*}{0,3} & 5 & 4,0088 & 0,2205 & 0,0143 & $-2,0226$ & 1,1305 & 0,039 & 0,4778 & $-4,4436$ & 0,0261 & 0,272 \\
\hline & 10 & 4,0051 & 0,128 & 0,0087 & & $-0,2158$ & & & $-1,0717$ & 0,0316 & 0,2805 \\
\hline & 3 & 4,008 & 0,2004 & 0,0286 & -2 , & 1,0702 & & & $-5,2003$ & 0,024 & 5599 \\
\hline \multirow[t]{3}{*}{0,6} & 5 & 4,022 & 0,5497 & 0,0258 & $-2,0041$ & 0,2042 & 0,0244 & 17 & $-3,653$ & 0,0247 & 5592 \\
\hline & 10 & 3,9732 & $-0,6712$ & 0,0136 & $-2,0022$ & 0,1093 & 0,0076 & 0,4862 & $-2,7591$ & 0,0228 & 0,5424 \\
\hline & 3 & 3,9887 & $-0,2835$ & 0,0183 & $-2,0022$ & 0,1089 & 0,0119 & 0,4796 & $-4,0783$ & 0,022 & 0,8858 \\
\hline \multirow[t]{4}{*}{0,9} & 5 & 4,0008 & 0,0207 & 0,0185 & $-1,9983$ & $-0,0841$ & 0,0057 & 0,4764 & $-4,7148$ & 0,026 & 0,8817 \\
\hline & 10 & 3,9906 & $-0,2361$ & 0,0156 & & 0,036 & 0,0022 & & $-1,4841$ & 0,0232 & 0,8866 \\
\hline & & \multicolumn{10}{|c|}{$n=50$} \\
\hline & 3 & 4,0038 & 943 & 0,0087 & $\overline{00}$ & 0,2007 & 0,0245 & 02 & 2,0425 & 0,0247 & 0,2702 \\
\hline \multirow[t]{3}{*}{0,3} & 5 & 3,9957 & $-0,1073$ & 0,0059 & $-1,9859$ & $-0,7063$ & 0,0135 & 0,4923 & $-1,5482$ & 0,0204 & 0,2874 \\
\hline & 10 & 4,0139 & 0,348 & 0,0047 & $-2,0168$ & 0,8392 & 0,0075 & & $-2,4449$ & 0,0227 & 0,2781 \\
\hline & 3 & 4,0084 & 0,2093 & 0,0099 & & $-0,5795$ & 55 & 0,4786 & $-4,2729$ & 0,016 & 0,5893 \\
\hline \multirow[t]{3}{*}{0,6} & 5 & 3,9894 & $-0,2644$ & 0,0071 & & $-0,3329$ & 85 & & $-3,3475$ & 0,0186 & 0,5619 \\
\hline & 10 & & & 0 , & & 06 & & & $-1,0282$ & 78 & 835 \\
\hline & 3 & 3,9806 & $-0,4845$ & 0,0066 & $-1,9851$ & $-0,7439$ & 0,0043 & 0,4851 & $-2,9764$ & 0,0259 & 0,8913 \\
\hline \multirow[t]{4}{*}{0,9} & 5 & 3,9988 & $-0,0308$ & 0,0063 & $-2,0102$ & 0,5125 & 0,002 & 0,4978 & $-0,433$ & 0,0272 & 0,8884 \\
\hline & 10 & 4,0062 & 0,1542 & 0,0064 & $-2,0045$ & 0,2249 & 0,0004 & 0,5207 & 4,1443 & 0,0407 & 0,8902 \\
\hline & & \multicolumn{10}{|c|}{$n=$} \\
\hline & 3 & 4,0097 & 0 , & 0, & & 90,3459 & 0,0 & & 2,8732 & 0,023 & 0,2836 \\
\hline \multirow[t]{3}{*}{0,3} & 5 & 4,009 & 0,2247 & 0,004 & $-2,0$ & 0,6983 & 0,0111 & & 4,0716 & 0,0303 & 0,2819 \\
\hline & 10 & 3,9952 & $-0,1195$ & 0,0025 & $-1,9927$ & $-0,3642$ & 0,0 & 0, & $-0,6126$ & 0,0225 & 0,2914 \\
\hline & 3 & 4,0044 & 0,11 & 0,0052 & $-2,0105$ & 0,5251 & 0,0085 & 0,4842 & $-3,1528$ & 0,0212 & 0,5787 \\
\hline 0,6 & 5 & 3,9944 & $-0,1404$ & 0,0034 & $-2,001$ & 0,0479 & 0,004 & 0,4875 & $-2,4977$ & 0,0135 & 0,5834 \\
\hline & 10 & 4,0098 & 0,2444 & 0,0032 & $-2,0061$ & 0,3072 & 0,0019 & 0,5221 & 4,4261 & 0,0348 & 0,5875 \\
\hline & 3 & 4,0096 & 0,2401 & 0,0043 & $-2,0054$ & 0,268 & 0,0031 & 0,476 & $-4,8012$ & 0,018 & 0,8868 \\
\hline 0,9 & 5 & 4,0051 & 0,1285 & 0,0055 & $-1,9991$ & $-0,0467$ & 0,0017 & 0,5022 & 0,4464 & 0,0297 & 0,8902 \\
\hline & 10 & 3,9981 & $-0,0469$ & 0,0033 & $-2,0035$ & 0,1744 & 0,0005 & 0,4869 & $-2,6281$ & 0,0319 & 0,8894 \\
\hline
\end{tabular}


Tabela E.5: Estimativas médias de $\beta_{0}, \beta_{1}, \alpha$ e $\rho$, viés relativo (VR) (em \%) e erro quadrático médio (EQM) de $\hat{\beta}_{0}$ e $\hat{\beta}_{1}$ do estudo de simulação dos quais os dados são gerados de uma distribuição multivariada $B S$ de uma estrutura de correlação AR-1 e ajustado sob o modelo log-BS-t via GEE com mesma estrutura de correlação verdadeira, $(\nu=7)$.

\begin{tabular}{|c|c|c|c|c|c|c|c|c|c|c|c|}
\hline \multirow{3}{*}{$\rho$} & \multirow{3}{*}{$\mathrm{s}$} & \multicolumn{3}{|c|}{$\hat{\beta}_{0}$} & \multirow[b]{2}{*}{$\beta_{1}$} & \multicolumn{2}{|c|}{$\hat{\beta}_{1}$} & \multirow[b]{2}{*}{$\alpha$} & \multicolumn{2}{|c|}{$\hat{\alpha}$} & \multirow[b]{2}{*}{$\rho$} \\
\hline & & $\beta_{0}$ & VR & EQM & & VR & EQM & & VR & EQM & \\
\hline & & \multicolumn{10}{|c|}{$n=10$} \\
\hline & 3 & 4,0132 & 0,3299 & 0,0603 & $-2,0233$ & 1,1642 & 0,1536 & 0,5107 & 2,1469 & 0,049 & 0,2186 \\
\hline \multirow[t]{3}{*}{0,3} & 5 & 3,999 & $-0,0259$ & 0,0468 & $-2,0024$ & 0,1204 & 0,1165 & 0,4933 & $-1,3361$ & 0,0268 & 0,256 \\
\hline & 10 & 4,0005 & 0,0129 & 0,02 & $-2,0008$ & 0,0379 & 0,0415 & 0,5 & $-0,0078$ & 0,0314 & 0,2966 \\
\hline & 3 & 4,0035 & 0,0887 & 0,0245 & $-2,0317$ & 1,5854 & 0,0659 & 0,4734 & $-5,3156$ & 0,0298 & 0,5479 \\
\hline \multirow[t]{3}{*}{0,6} & 5 & 4,0257 & 0,6414 & 0,0226 & $-2,0242$ & 1,2086 & 0,0478 & 0,4629 & $-7,4276$ & 0,0217 & 0,5637 \\
\hline & 10 & 4,0076 & 0,1909 & 0,0127 & $-1,9775$ & $-1,1234$ & 0,0191 & 0,4957 & $-0,8541$ & 0,0262 & 0,558 \\
\hline & 3 & 3,997 & $-0,0744$ & 0,0468 & $-1,9967$ & $-0,1645$ & 0,0224 & 0,4707 & $-5,864$ & 0,0325 & 0,8731 \\
\hline \multirow[t]{4}{*}{0,9} & 5 & 4,014 & 0,349 & 0,0343 & $-1,9889$ & $-0,5558$ & 0,0084 & 0,4852 & $-2,9532$ & 0,0364 & 0,8759 \\
\hline & 10 & 3,9875 & $-0,3135$ & 0,0189 & $-1,9978$ & $-0,1102$ & 0,0043 & 0,5018 & 0,3681 & 0,0507 & 0,8867 \\
\hline & & \multicolumn{10}{|c|}{$n=20$} \\
\hline & 3 & 4,013 & 0,3255 & 0,0247 & $-2,0126$ & 0,6317 & 0,0895 & 0,507 & 1,4079 & 0,0458 & 0,2826 \\
\hline \multirow[t]{3}{*}{0,3} & 5 & 4,0091 & 0,2285 & 0,0135 & $-2,0218$ & 1,0877 & 0,0406 & 0,479 & $-4,1972$ & 0,0255 & 0,2774 \\
\hline & 10 & 4,0045 & 0,1121 & 0,0067 & $-1,9993$ & $-0,0349$ & 0,0216 & 0,4975 & $-0,5081$ & 0,0332 & 0,2894 \\
\hline & 3 & 4,0084 & 0,2102 & 0,0276 & $-2,0201$ & 1,0058 & 0,0244 & 0,4747 & $-5,0642$ & 0,0238 & 0,5676 \\
\hline \multirow[t]{3}{*}{0,6} & 5 & 4,0217 & 0,5437 & 0,0227 & $-2,0071$ & 0,3554 & 0,027 & 0,4853 & $-2,9434$ & 0,0241 & 0,5692 \\
\hline & 10 & 3,9861 & $-0,3485$ & 0,0075 & $-2,0085$ & 0,4262 & 0,008 & 0,4968 & $-0,6491$ & 0,0213 & 0,5711 \\
\hline & 3 & 3,9866 & $-0,3355$ & 0,0187 & $-2,0012$ & 0,0584 & 0,012 & 0,4801 & $-3,9842$ & 0,0218 & 0,8879 \\
\hline \multirow[t]{4}{*}{0,9} & 5 & 4,0008 & 0,0189 & 0,0174 & $-1,9982$ & $-0,0924$ & 0,0056 & 0,4769 & $-4,6156$ & 0,0255 & 0,8837 \\
\hline & 10 & 3,9944 & $-0,1405$ & 0,0129 & $-1,9992$ & $-0,0381$ & 0,0022 & 0,4961 & $-0,7775$ & 0,0223 & 0,8919 \\
\hline & & \multicolumn{10}{|c|}{$n=50$} \\
\hline & 3 & 4,0019 & 0,0465 & 0,0084 & $-2,0001$ & 0,0059 & 0,0245 & 0,5112 & 2,2355 & 0,0247 & 0,2743 \\
\hline \multirow[t]{3}{*}{0,3} & 5 & 3,9955 & $-0,1126$ & 0,0054 & $-1,9852$ & $-0,7379$ & 0,0149 & 0,4928 & $-1,4437$ & 0,0205 & 0,2928 \\
\hline & 10 & 4,0108 & 0,2711 & 0,0036 & $-2,0148$ & 0,7411 & 0,0082 & 0,4913 & $-1,7361$ & 0,023 & 0,2917 \\
\hline & 3 & 4,0087 & 0,2186 & 0,01 & $-1,9895$ & $-0,5248$ & 0,0162 & 0,4789 & $-4,2214$ & 0,016 & 0,5913 \\
\hline \multirow[t]{3}{*}{0,6} & 5 & 3,9913 & $-0,2168$ & 0,0061 & $-1,995$ & $-0,2512$ & 0,0091 & 0,4867 & $-2,6645$ & 0,0182 & 0,5691 \\
\hline & 10 & 4,0007 & 0,0163 & 0,0033 & $-1,9983$ & $-0,087$ & 0,0038 & 0,4961 & $-0,7755$ & 0,0285 & 0,5886 \\
\hline & 3 & 3,979 & $-0,5249$ & 0,0064 & $-1,9828$ & $-0,8586$ & 0,0042 & 0,4851 & $-2,988$ & 0,0257 & 0,8919 \\
\hline \multirow[t]{4}{*}{0,9} & 5 & 3,9979 & $-0,0532$ & 0,0055 & $-2,0092$ & 0,4593 & 0,0019 & 0,4988 & $-0,2307$ & 0,0268 & 0,8902 \\
\hline & 10 & 4,0035 & 0,0863 & 0,0051 & $-2,0036$ & 0,1811 & 0,0009 & 0,5219 & 4,381 & 0,0411 & 0,8921 \\
\hline & & \multicolumn{10}{|c|}{$n=80$} \\
\hline & 3 & 4,0091 & 0,2283 & 0,0068 & $-2,006$ & 0,2986 & 0,0159 & 0,515 & 3,0019 & 0,0231 & 0,2873 \\
\hline \multirow[t]{3}{*}{0,3} & 5 & 4,0103 & 0,2585 & 0,004 & $-2,0167$ & 0,8347 & 0,0125 & 0,5214 & 4,2721 & 0,0297 & 0,2878 \\
\hline & 10 & 3,9949 & $-0,1273$ & 0,0019 & $-1,9917$ & $-0,4152$ & 0,0051 & 0,4969 & $-0,6161$ & 0,0228 & 0,2897 \\
\hline & 3 & 4,0034 & 0,0853 & 0,0048 & $-2,0076$ & 0,3796 & 0,009 & 0,4845 & $-3,108$ & 0,0211 & 0,5795 \\
\hline 0,6 & 5 & 3,9973 & $-0,0674$ & 0,0028 & $-2,0045$ & 0,2249 & 0,0041 & 0,4885 & $-2,2993$ & 0,0134 & 0,5878 \\
\hline & 10 & 4,0077 & 0,1922 & 0,002 & $-2,0055$ & 0,2733 & 0,0023 & 0,522 & 4,3905 & 0,0349 & 0,5883 \\
\hline & 3 & 4,0083 & 0,2076 & 0,0042 & $-2,0027$ & 0,1343 & 0,0031 & 0,4767 & $-4,6697$ & 0,018 & 0,8876 \\
\hline 0,9 & 5 & 4,0047 & 0,1185 & 0,0048 & $-1,9995$ & $-0,0239$ & 0,0016 & 0,5021 & 0,4244 & 0,0293 & 0,8904 \\
\hline & 10 & 3,9995 & $-0,0126$ & 0,0025 & $-2,0022$ & 0,1086 & 0,0005 & 0,4884 & $-2,328$ & 0,0316 & 0,891 \\
\hline
\end{tabular}


Tabela E.6: Estimativas médias de $\beta_{0}, \beta_{1}, \alpha$ e $\rho$, viés relativo (VR) (em \%) e erro quadrático médio (EQM) de $\hat{\beta}_{0}$ e $\hat{\beta}_{1}$ do estudo de simulação dos quais os dados são gerados utilizando uma estrutura de correlação $A R-1$ e ajustado sob o modelo log-BS-t via GEE estrutura de correlação permutável, $(\nu=7)$.

\begin{tabular}{|c|c|c|c|c|c|c|c|c|c|c|c|}
\hline \multirow{3}{*}{$\rho$} & \multirow{3}{*}{$\mathrm{s}$} & \multicolumn{3}{|c|}{$\hat{\beta}_{0}$} & \multirow[b]{2}{*}{$\beta_{1}$} & \multicolumn{2}{|c|}{$\hat{\beta}_{1}$} & \multirow[b]{2}{*}{$\alpha$} & \multicolumn{2}{|c|}{ o } & \multirow[b]{2}{*}{$\rho$} \\
\hline & & $\beta_{0}$ & VR & EQM & & VR & EQM & & VR & EQM & \\
\hline & & \multicolumn{10}{|c|}{$n=10$} \\
\hline & 3 & 4,0057 & 0,1416 & 0,0494 & $-1,984$ & $-0,798$ & 0,133 & 0,4771 & $-4,5773$ & 0,026 & 0,2111 \\
\hline \multirow[t]{3}{*}{0,3} & 5 & 4,0006 & 0,016 & 0,0328 & $-1,9808$ & $-0,9595$ & 0,0909 & 0,4854 & $-2,923$ & 0,0281 & 0,0998 \\
\hline & 10 & 4,0306 & 0,901 & 0,0217 & $-2,0606$ & 3,0316 & 0,061 & 0,4942 & $-1,1663$ & 0,0296 & 0,0722 \\
\hline & 3 & 3,9873 & $-0,3184$ & 0,0415 & $-1,9814$ & $-0,9301$ & 0,0922 & 0,4925 & $-1,4997$ & 0,0464 & 0,4438 \\
\hline \multirow[t]{3}{*}{0,6} & 5 & 3,983 & $-0,4248$ & 0,0393 & $-1,9786$ & $-1,0686$ & 0,0853 & 0,4824 & $-3,5285$ & 0,0378 & 0,3509 \\
\hline & 10 & 4,0095 & 0,2378 & 0,0147 & $-1,9953$ & $-0,2373$ & 0,0355 & 4695 & $-6,1066$ & ,0209 & 0,2154 \\
\hline & 3 & 4,0485 & 1,2117 & 0,0485 & $-2,0384$ & 1,9193 & 0,0307 & 0,4864 & $-2,7231$ & 0,0303 & 0,8481 \\
\hline \multirow[t]{4}{*}{0,9} & 5 & 4,0257 & 0,6423 & 0,0305 & $-2,0163$ & 0,8128 & 0,013 & 0,4348 & $-13,0353$ & 0,028 & 0,7666 \\
\hline & 10 & 3,9967 & $-0,0828$ & 0,0223 & $-2,0055$ & 0,274 & 0,0122 & 0,4849 & $-3,0254$ & 0,0294 & 0,6387 \\
\hline & & \multicolumn{10}{|c|}{$n=20$} \\
\hline & 3 & 4,0303 & 0,7574 & 0,0216 & 0708 & 3,5387 & 0,0766 & 0,4842 & 3,1611 & 0302 & 0,1705 \\
\hline \multirow[t]{3}{*}{0,3} & 5 & 3,9959 & $-0,103$ & 0,0143 & $-2,017$ & 0,8497 & 0,044 & & $-0,1944$ & ,0238 & 0,1116 \\
\hline & 10 & 4,0023 & 0,0584 & 0,0076 & $-1,9993$ & $-0,035$ & 0,0249 & & $-1,8305$ & 0,0259 & ,079 \\
\hline & 3 & 3,9985 & $-0,0371$ & 0,0134 & $-2,0058$ & 0,2889 & 0,0339 & 32 & $-0,3643$ & 0,0416 & 4862 \\
\hline \multirow[t]{3}{*}{0,6} & 5 & 4,0246 & 0,6156 & 0,0186 & $-2,0513$ & 2,5653 & 0,0323 & 4807 & $-3,8523$ & 0,0187 & 0,3635 \\
\hline & 10 & 4,0111 & 0,2774 & 0,0077 & $-2,0114$ & 0,5677 & 0,0116 & 0,4878 & $-2,4432$ & 0,0267 & 0,2301 \\
\hline & 3 & 4,0103 & 0,2575 & 0,0191 & $-2,0309$ & 1,5469 & 0,0132 & 0,479 & $-4,1948$ & 0,0206 & 0,8495 \\
\hline \multirow[t]{4}{*}{0,9} & 5 & 4,01 & 0,2508 & 0,0155 & $-1,9809$ & $-0,9543$ & 0,0141 & 0,4 & $-1,9178$ & 0,0272 & 0,7923 \\
\hline & 10 & 3,9976 & $-0,061$ & 0,0169 & $-1,994$ & $-0,2991$ & 0,0067 & 0,5038 & 0,7517 & 0,028 & 0,6745 \\
\hline & & \multicolumn{10}{|c|}{$n=50$} \\
\hline & 3 & 4,0074 & 1851 & 0,0063 & 1 & 0,0044 & 0,0205 & & 0,0403 & 0286 & 0,2048 \\
\hline \multirow[t]{3}{*}{0,3} & 5 & 3,9935 & $-0,1633$ & 0,0062 & $-1,9725$ & $-1,374$ & 0,0153 & 0,5007 & 0,1432 & 0,0245 & 0,14 \\
\hline & 10 & 4,0055 & 0,1386 & 0,0033 & $-2,0025$ & 0,1253 & 0,0073 & 0,5263 & 5,2667 & 0,0419 & 0,0754 \\
\hline & 3 & 4,0094 & 0,2343 & 0,0073 & & 1,2909 & 0,0111 & & 3,7786 & 0,0357 & 0,4931 \\
\hline \multirow[t]{3}{*}{0,6} & 5 & 3,9961 & $-0,0985$ & 0,0052 & -1 , & $-0,864$ & 0,0119 & 0 & 0,3879 & 0,0217 & 0,3735 \\
\hline & 10 & 4,0027 & 0,0679 & 0,0041 & $-2,0053$ & 0,2674 & 0,0057 & 0,4850 & $-3,0093$ & 0,0428 & 0,227 \\
\hline & 3 & 4,001 & 0,0247 & 0,0074 & $-2,0058$ & 0,2899 & 0,0046 & 0,4963 & $-0,7485$ & 0,0327 & 0,8558 \\
\hline \multirow[t]{4}{*}{0,9} & 5 & 4,0018 & 0,0453 & 0,006 & $-1,9979$ & $-0,1069$ & 0,0042 & 0,5001 & 0,0226 & 0,024 & 0,7993 \\
\hline & 10 & 3,9991 & $-0,0223$ & 0,0042 & $-2,0014$ & 0,0720 & 0,002 & 0,4622 & $-7,5682$ & 0,0167 & 0,6779 \\
\hline & & \multicolumn{10}{|c|}{$n=80$} \\
\hline & 3 & 3 & 88 & 0 , & 08 & $-0,539$ & 0,014 & & $-1,703$ & 169 & 2143 \\
\hline \multirow[t]{3}{*}{0,3} & 5 & 3,9942 & $-0,1448$ & 0,0034 & $-1,9839$ & $-0,805$ & 0,0084 & 0,4 & $-0,218$ & 0,0279 & 0,1412 \\
\hline & 10 & 3,9991 & $-0,0231$ & 0,002 & $-2,0002$ & 0,0083 & 0,0051 & 0,5 & 1,1667 & 0,0273 & 0,072 \\
\hline & 3 & 3,9897 & $-0,2575$ & 0,0065 & $-1,9935$ & $-0,3238$ & 0,0137 & 0,5283 & 5,655 & 0,0359 & 0,5018 \\
\hline 0,6 & 5 & 3,988 & $-0,3011$ & 0,0045 & $-1,9876$ & $-0,6224$ & 0,0068 & 0,504 & 0,7915 & 0,0233 & 0,3875 \\
\hline & 10 & 3,9995 & $-0,0124$ & 0,0025 & $-2,0000$ & $-0,0003$ & 0,0036 & 0,4841 & $-3,1725$ & 0,0194 & 0,2361 \\
\hline & 3 & 3,9934 & $-0,1657$ & 0,0041 & $-1,9922$ & $-0,3901$ & 0,0029 & 0,4 ! & $-0,8429$ & 0,027 & 0,8629 \\
\hline 0,9 & 5 & 4,0011 & 0,0276 & 0,0032 & $-1,999$ & $-0,0516$ & 0,0025 & 0,5081 & 1,6287 & 0,0232 & 0,8073 \\
\hline & 10 & 3,9924 & $-0,1893$ & 0,0029 & $-2,0012$ & 0,0599 & 0,0018 & 0,5138 & 2,7514 & 0,0274 & 0,6806 \\
\hline
\end{tabular}


Tabela E.7: Estimativas médias de $\beta_{0}, \beta_{1}, \alpha$ e $\rho$, viés relativo (VR) (em \%) e erro quadrático médio (EQM) de $\hat{\beta}_{0}$ e $\hat{\beta}_{1}$ do estudo de simulação dos quais os dados são gerados de uma distribuição multivariada BS de uma estrutura de correlação permutável e ajustado sob o modelo log-BS-t-GEE models com mesma estrutura de correlação $(\nu=10)$.

\begin{tabular}{|c|c|c|c|c|c|c|c|c|c|c|c|}
\hline \multirow{3}{*}{$\rho$} & \multirow{3}{*}{$\mathrm{s}$} & \multicolumn{3}{|c|}{$\hat{\beta}_{0}$} & \multirow[b]{2}{*}{$\beta_{1}$} & \multicolumn{2}{|c|}{$\hat{\beta}_{1}$} & \multirow[b]{2}{*}{$\alpha$} & \multicolumn{2}{|c|}{$\alpha$} & \multirow[b]{2}{*}{$\rho$} \\
\hline & & $\beta_{0}$ & VR & EQM & & VR & EQM & & VR & EQM & \\
\hline & & \multicolumn{10}{|c|}{$n=10$} \\
\hline & 3 & 3,9975 & $-0,0637$ & 0,0373 & $-2,0006$ & 0,0279 & 0,1096 & 0,4694 & $-6,1189$ & 0,0199 & 0,2427 \\
\hline \multirow[t]{3}{*}{0,3} & 5 & 4,0023 & 0,0583 & 0,0281 & $-2,0003$ & 0,0131 & 0,0590 & 0,4754 & $-4,9205$ & 0,0179 & 0,2529 \\
\hline & 10 & 4,0027 & 0,0663 & 0,0167 & $-2,0028$ & 0,1402 & 0,0260 & 0,4794 & $-4,1127$ & 0,0174 & 0,2604 \\
\hline & 3 & 3,9988 & $-0,0301$ & 0,0444 & $-1,9999$ & $-0,0046$ & 0,0736 & 0,4633 & $-7,3327$ & 0,0214 & 0,5419 \\
\hline \multirow[t]{3}{*}{0,6} & 5 & 3,9963 & $-0,0925$ & 0,0292 & $-2,0017$ & 0,0851 & 0,0354 & 0,4721 & $-5,5809$ & 0,0207 & 0,5433 \\
\hline & 10 & 4,0009 & 0,0231 & 0,0230 & $-2,0024$ & 0,1216 & 0,0177 & 0,4773 & $-4,5387$ & 0,0200 & 0,5458 \\
\hline & 3 & 3,9974 & $-0,0660$ & 0,0331 & $-2,0007$ & 0,0359 & 0,0254 & 0,4507 & $-9,8640$ & 0,0254 & 0,8720 \\
\hline \multirow[t]{4}{*}{0,9} & 5 & 3,9990 & $-0,0246$ & 0,0290 & $-2,0010$ & 0,0504 & 0,0098 & 0,4668 & $-6,6337$ & 0,0271 & 0,8701 \\
\hline & 10 & 4,0005 & 0,0121 & 0,0283 & $-1,9998$ & $-0,0076$ & 0,0039 & 0,4695 & $-6,1040$ & 0,0271 & 0,8708 \\
\hline & & \multicolumn{10}{|c|}{$n=20$} \\
\hline & 3 & 3,9991 & $-0,0213$ & 0,0223 & $-2,0023$ & 0,1164 & 0,0515 & 0,4860 & $-2,8000$ & 0,0187 & 0,2689 \\
\hline \multirow[t]{3}{*}{0,3} & 5 & 4,0020 & 0,0502 & 0,0149 & $-2,0054$ & 0,2723 & 0,0352 & 0,4896 & $-2,0841$ & 0,0173 & 0,2733 \\
\hline & 10 & 4,0022 & 0,0555 & 0,0088 & $-2,0033$ & 0,1653 & 0,0135 & 0,4924 & $-1,5176$ & 0,0163 & 0,2786 \\
\hline & 3 & 3,9964 & $-0,0911$ & 0,0191 & $-1,9951$ & $-0,2453$ & 0,0398 & 0,4 & $-4,2380$ & 0,0186 & 0,5662 \\
\hline \multirow[t]{3}{*}{0,6} & 5 & 4,0005 & 0,0118 & 0,0144 & $-1,9992$ & $-0,0389$ & 0,0182 & 0,4845 & $-3,0978$ & 0,0186 & 0,5722 \\
\hline & 10 & 4,0008 & 0,0194 & 0,0117 & $-1,9997$ & $-0,0145$ & 0,0096 & 0,4861 & $-2,7859$ & 0,0179 & 0,5688 \\
\hline & 3 & 4,0014 & 0,0353 & 0,0163 & $-1,9998$ & $-0,0118$ & 0,0113 & 0,4741 & $-5,1841$ & 0,0199 & 0,8847 \\
\hline \multirow[t]{4}{*}{0,9} & 5 & 3,9978 & $-0,0542$ & 0,0143 & $-2,0001$ & 0,0056 & 0,0039 & 0,4793 & $-4,1415$ & 0,0209 & 0,8842 \\
\hline & 10 & 4,0018 & 0,0443 & 0,0141 & $-2,0002$ & 0,0098 & 0,0023 & 0,4825 & $-3,5053$ & 0,0206 & 0,8840 \\
\hline & & \multicolumn{10}{|c|}{$n=50$} \\
\hline & 3 & 3,9980 & $-0,0499$ & 0,0081 & $-1,9966$ & $-0,1681$ & 0,0209 & 0,4917 & $-1,6677$ & 0,0165 & 0,2862 \\
\hline \multirow[t]{3}{*}{0,3} & 5 & 4,0013 & 0,0329 & 0,0057 & $-2,0017$ & 0,0862 & 0,0120 & 0,4925 & $-1,4946$ & 0,0162 & 0,2882 \\
\hline & 10 & 4,0008 & 0,0205 & 0,0035 & $-2,0015$ & 0,0758 & 0,0055 & 0,4959 & $-0,8254$ & 0,0171 & 0,2893 \\
\hline & 3 & 3,9982 & $-0,0439$ & 0,0072 & $-1,9981$ & $-0,0953$ & 0,0122 & 0,4896 & $-2,0821$ & 0,0180 & 0,5857 \\
\hline \multirow{3}{*}{0,6} & 5 & 3,9992 & $-0,0201$ & 0,0057 & $-1,9999$ & $-0,0059$ & 0,0070 & 0,4921 & $-1,5847$ & 0,0168 & 0,5832 \\
\hline & 10 & 3,9998 & $-0,0044$ & 0,0043 & -1 , & $-0,0421$ & 0,0030 & 0, & -1 , & 0,0169 & 0,5821 \\
\hline & 3 & 4,0000 & $-0,0010$ & 0,0065 & $-2,0009$ & 0,0456 & 0,0032 & 0,4912 & $-1,7532$ & 0,0190 & 0,8927 \\
\hline \multirow[t]{4}{*}{0,9} & 5 & 4,0008 & 0,0194 & 0,0060 & $-2,0010$ & 0,0520 & 0,0017 & 0,4916 & $-1,6712$ & 0,0173 & 0,8931 \\
\hline & 10 & 4,0021 & 0,0527 & 0,0055 & $-1,9999$ & $-0,0073$ & 0,0009 & 0,4918 & $-1,6431$ & 0,0180 & 0,8926 \\
\hline & & \multicolumn{10}{|c|}{$n=$} \\
\hline & 3 & 4,0026 & 0,0660 & 0,0049 & $-2,0026$ & 0,1322 & 0,0126 & 0,4940 & $-1,2021$ & 0,0165 & 0,2899 \\
\hline \multirow[t]{3}{*}{0,3} & 5 & 3,9997 & $-0,0081$ & 0,0034 & $-2,0009$ & 0,0471 & 0,0075 & 0,4955 & $-0,8978$ & 0,0170 & 0,2899 \\
\hline & 10 & 3,9998 & $-0,0041$ & 0,0022 & $-2,0004$ & 0,0197 & 0,0034 & 0,4983 & $-0,3311$ & 0,0175 & 0,2934 \\
\hline & 3 & 4,0007 & 0,0185 & 0,0051 & $-2,0007$ & 0,0334 & 0,0086 & 0,4916 & $-1,6775$ & 0,0162 & 0,5875 \\
\hline 0,6 & 5 & 3,9995 & $-0,0117$ & 0,0037 & $-1,9995$ & $-0,0251$ & 0,0047 & 0,4955 & $-0,9029$ & 0,0170 & 0,5876 \\
\hline & 10 & 3,9995 & $-0,0127$ & 0,0028 & $-1,9999$ & $-0,0037$ & 0,0019 & 0,4924 & $-1,5299$ & 0,0160 & 0,5887 \\
\hline & 3 & 4,0010 & 0,0254 & 0,0040 & $-2,0008$ & 0,0425 & 0,0023 & 0,4923 & $-1,5377$ & 0,0166 & 0,8942 \\
\hline 0,9 & 5 & 3,9993 & $-0,0183$ & 0,0036 & $-2,0001$ & 0,0069 & 0,0011 & 0,4944 & $-1,1192$ & 0,0172 & 0,8949 \\
\hline & 10 & 4,0005 & 0,0123 & 0,0035 & $-2,0003$ & 0,0162 & 0,0005 & 0,4966 & $-0,6894$ & 0,0176 & 0,8946 \\
\hline
\end{tabular}


Tabela E.8: Estimativas médias de $\beta_{0}, \beta_{1}, \alpha$ e $\rho$, viés relativo (VR) (em \%) e erro quadrático médio (EQM) de $\hat{\beta}_{0}$ e $\hat{\beta}_{1}$ do estudo de simulação dos quais os dados são gerados de uma distribuição multivariada BS de uma estrutura de correlação permutável e ajustado sob o modelo log-BS-t via GEE com mesma estrutura de correlação $(\nu=30)$.

\begin{tabular}{|c|c|c|c|c|c|c|c|c|c|c|c|}
\hline \multirow{3}{*}{$\rho$} & \multirow{3}{*}{$\mathrm{s}$} & \multicolumn{3}{|c|}{$\hat{\beta}_{0}$} & \multirow[b]{2}{*}{$\beta_{1}$} & \multicolumn{2}{|c|}{$\hat{\beta}_{1}$} & \multirow[b]{2}{*}{$\alpha$} & \multicolumn{2}{|c|}{$\alpha$} & \multirow[b]{2}{*}{$\rho$} \\
\hline & & $\beta_{0}$ & VR & EQM & & VR & EQM & & VR & EQM & \\
\hline & & \multicolumn{10}{|c|}{$n=10$} \\
\hline & 3 & 3 , & $-0,0352$ & 0 , & 4 & 318 & 0,0 & 0,4666 & 6841 &, 0095 & 2406 \\
\hline \multirow[t]{3}{*}{0,3} & 5 & 3,9964 & $-0,0896$ & 0,0252 & $-1,9945$ & $-0,2734$ & 0,0520 & 0,4766 & $-4,6807$ & 0,0076 & 0,2543 \\
\hline & 10 & 4,0003 & 0,0063 & 0,0151 & $-2,0008$ & 0,0410 & 0,0227 & 0,4816 & $-3,6701$ & 0,0066 & 0,2618 \\
\hline & 3 & 4,0013 & 0,0315 & 0,0344 & $-2,0033$ & 0,1641 & 0,0597 & 0,4648 & $-7,0428$ & 0,0118 & 0,5458 \\
\hline \multirow[t]{3}{*}{0,6} & 5 & 4,0022 & 0,0554 & 0,0235 & $-2,0039$ & 0,1928 & 0,0283 & 0,4722 & $-5,5510$ & 0,0108 & 0,5454 \\
\hline & 10 & 3,9984 & $-0,0390$ & 0,0195 & $-2,0007$ & 0,0372 & 0,0127 & 0,4719 & $-5,6253$ & 0,0094 & 0,5412 \\
\hline & 3 & 4,0003 & 0,0087 & 0,0278 & $-1,9984$ & $-0,0820$ & 0,0169 & & $-10,0401$ & 0,0164 & 0,8715 \\
\hline \multirow[t]{4}{*}{0,9} & 5 & 3,9965 & $-0,0879$ & 0,0254 & $-1,9973$ & $-0,1357$ & 0,0077 & 87 & 8,2671 & 0,0152 & 0,8711 \\
\hline & 10 & 3,9986 & $-0,0345$ & 0,0232 & $-1,9993$ & $-0,0332$ & 0,0029 & 0,4622 & 7,5522 & 0,0149 & 0,8690 \\
\hline & & \multicolumn{10}{|c|}{$n=20$} \\
\hline & 3 & 3,9999 & $-0,0035$ & 0,0158 & $-2,0026$ & 0,1311 & 0,0389 & 0,4 & $-3,3186$ & 0,0068 & 0,2727 \\
\hline \multirow{3}{*}{0,3} & 5 & 4,0006 & 0,0143 & 0,0112 & $-1,9983$ & $-0,0850$ & 0,0252 & & $-2,6709$ & 0,0062 & 0,2756 \\
\hline & 10 & 4,0020 & 0,0507 & 0,0073 & & 0,0889 & & & $-2,0490$ & & 0,2790 \\
\hline & 3 & 3,9997 & $-0,0070$ & 0,0 & & 0,0471 & 0,0 & & 157 & & 0,5718 \\
\hline \multirow[t]{3}{*}{0,6} & 5 & 4,0000 & $-0,0009$ & 0,01 & $-1,9989$ & $-0,0547$ & 0,0164 & & $-3,1800$ & 0,0075 & 0,5719 \\
\hline & 10 & 3,9992 & $-0,0190$ & 0,0096 & $-1,9990$ & $-0,0519$ & 0,0066 & 19 & $-3,0160$ & 0,0070 & 0,5721 \\
\hline & 3 & 3,9980 & $-0,0493$ & 0,0137 & $-1,9986$ & $-0,0712$ & 0,0086 & 17 & $-5,0501$ & 0,0098 & 0,8864 \\
\hline \multirow[t]{4}{*}{0,9} & 5 & 4,0030 & 0,0753 & 0,0128 & $-2,0010$ & 0,0523 & 0,0035 & 0,4 & $-4,5079$ & 0,0098 & 0,8863 \\
\hline & 10 & 3,9984 & $-0,0407$ & 0,0119 & & $-0,0281$ & 0,0016 & & $-3,8350$ & 0,0096 & 0,8863 \\
\hline & & \multicolumn{10}{|c|}{$n=50$} \\
\hline & 3 & 3,9991 &, 0218 & 0,0076 & 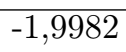 & $-0,0891$ & 0,0162 & 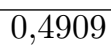 & 204 & 0,0055 & 0,2895 \\
\hline \multirow[t]{3}{*}{0,3} & 5 & 3,9988 & $-0,0292$ & 0,0049 & $-2,0003$ & 0,0143 & 0,0106 & & $-1,6295$ & 0,0052 & 0,2919 \\
\hline & 10 & 3,9995 & $-0,0123$ & 0,0030 & $-1,9999$ & $-0,0029$ & 0,0043 & & $-0,6489$ & 0,0051 & 0,2914 \\
\hline & 3 & 4,0001 & 0,0032 & 0,0062 & $-2,0005$ & 0,0250 & 0,0113 & & $-1,7344$ & 0,0058 & 0,5903 \\
\hline \multirow[t]{3}{*}{0,6} & 5 & 3,9973 & $-0,0664$ & 0,0052 & $-1,9981$ & $-0,0925$ & 0,0059 & & 297 & 0, & 0,5880 \\
\hline & 10 & & -0 & & & 36 & & & 581 & 0, & 92 \\
\hline & 3 & 3,9998 & $-0,0059$ & 0,0052 & $-2,0008$ & 0,0422 & 0,0026 & 0,4 & $-1,9097$ & 0,0066 & 0,8951 \\
\hline \multirow[t]{4}{*}{0,9} & 5 & 3,9998 & $-0,0059$ & 0,0050 & $-2,0003$ & 0,0173 & 0,0016 & 0,4913 & $-1,7475$ & 0,0065 & 0,8954 \\
\hline & 10 & 4,0003 & 0,0071 & 0,0048 & $-2,0004$ & 0,0182 & 0,0007 & 0,4899 & $-2,0185$ & 0,0064 & 0,8949 \\
\hline & & \multicolumn{10}{|c|}{$n=80$} \\
\hline & 3 & 3,99 & & & & 0,0710 & 0,0 & & 518 & & 0,2926 \\
\hline \multirow[t]{3}{*}{0,3} & 5 & 3,998 & -0, & 0,0 & $-1,9$ & $-0,0343$ & 0,0 & & 440 & & \\
\hline & 10 & 3,9997 & $-0,0069$ & 0,0020 & $-2,0001$ & 0,0043 & 0,0030 & 0 & $-0,6900$ & 0,0047 & 0,2947 \\
\hline & 3 & 4,0006 & 0,0158 & 0,0044 & $-2,0010$ & 0,0520 & 0,0076 & 04 & $-1,1484$ & 0,0052 & 0,5938 \\
\hline 0,6 & 5 & 3,9989 & $-0,0268$ & 0,0031 & $-1,9997$ & $-0,0161$ & 0,0038 & 0,4935 & $-1,2973$ & 0,0053 & 0,5941 \\
\hline & 10 & 3,9997 & $-0,0070$ & 0,0024 & $-2,0004$ & 0,0222 & 0,0016 & 0,4936 & $-1,2830$ & 0,0052 & 0,5928 \\
\hline & 3 & 3,9996 & $-0,0090$ & 0,0035 & $-1,9993$ & $-0,0351$ & 0,0021 & 0,4910 & $-1,8098$ & 0,0060 & 0,8963 \\
\hline 0,9 & 5 & 3,9977 & $-0,0565$ & 0,0031 & $-1,9995$ & $-0,0238$ & 0,0009 & 0,4922 & $-1,5536$ & 0,0056 & 0,8971 \\
\hline & 10 & 3,9997 & $-0,0082$ & 0,0030 & $-1,9999$ & $-0,0067$ & 0,0004 & 0,4939 & $-1,2197$ & 0,0057 & 0,8971 \\
\hline
\end{tabular}




\section{Apêndice F}

\section{Cálculos do Capítulo 4}

F.1 Função de estimação conjunta para o modelo heterogêneo logBS

\section{F.1.1 Derivação da função de estimação para o modelo do parâmetro de forma}

Iremos apresentar nesta seção os cálculos detalhados para a obtenção da função de estimação descrita em (4.2).

Temos que

$$
v_{i j}=\frac{1}{\alpha_{i j}}\left[\frac{4}{\alpha_{i j}^{2}} \operatorname{senh}^{2}\left(\frac{y_{i j}-\mu_{i j}}{2}\right)-1\right] .
$$

Logo,

$$
\begin{aligned}
\dot{v}_{i j}=\frac{\partial v_{i j}}{\partial \alpha_{i j}} & =-\frac{1}{\left(\alpha_{i j}\right)^{2}}\left[\frac{4}{\alpha_{i j}^{2}} \operatorname{senh}^{2}\left(\frac{y_{i j}-\mu_{i j}}{2}\right)-1\right]+\frac{1}{\alpha_{i j}}\left[-\frac{8}{\alpha_{i j}^{3}} \operatorname{senh}^{2}\left(\frac{y_{i j}-\mu_{i j}}{2}\right)\right] \\
& =-\frac{12}{\alpha_{i j}^{4}} \operatorname{senh}^{2}\left(\frac{y_{i j}-\mu_{i j}}{2}\right)+\frac{1}{\alpha_{i j}^{2}}
\end{aligned}
$$

de (B.1) segue que,

$$
\begin{aligned}
\mathrm{E}\left(\frac{\partial v_{i j}}{\partial \alpha_{i j}}\right) & =\mathrm{E}\left(-\frac{12}{\alpha_{i j}^{4}} \operatorname{senh}^{2}\left(\frac{y_{i j}-\mu_{i j}}{2}\right)+\frac{1}{\alpha_{i j}^{2}}\right) \\
& =-\frac{12}{\alpha_{i j}^{4}} \mathrm{E}\left(\operatorname{senh}^{2}\left(\frac{y_{i j}-\mu_{i j}}{2}\right)\right)+\frac{1}{\alpha_{i j}^{2}} \\
& =-\frac{12}{\alpha_{i j}^{4}} \times \frac{\alpha_{i j}^{2}}{4}+\frac{1}{\alpha_{i j}^{2}}=-\frac{2}{\alpha_{i j}^{2}} .
\end{aligned}
$$

Denotamos,

$$
\mathbf{M}_{i}=\mathrm{E}\left(\frac{\partial \mathbf{v}_{i}}{\partial \boldsymbol{\alpha}_{i}}\right)=\operatorname{diag}\left\{\mathrm{E}\left(\dot{v}_{i 1}\right), \ldots, \mathrm{E}\left(\dot{v}_{i s}\right)\right\}=\operatorname{diag}\left(-\frac{2}{\alpha_{i j}^{2}}, \ldots,-\frac{2}{\alpha_{i s}^{2}}\right),
$$


então,

$$
\mathrm{E}\left(\frac{\partial \mathbf{v}_{i}}{\partial \boldsymbol{\gamma}^{\top}}\right)^{\top}=\mathrm{E}\left(\frac{\partial \mathbf{v}_{i}}{\partial \boldsymbol{\alpha}_{i}} \frac{\partial \boldsymbol{\alpha}_{i}}{\partial \boldsymbol{\delta}_{i}} \frac{\partial \boldsymbol{\delta}_{i}}{\partial \boldsymbol{\gamma}^{\top}}\right)^{\top}=\mathbf{Z}_{i}^{\top} \mathbf{G}_{i} \mathbf{M}_{i}
$$

$\operatorname{com} \mathbf{G}_{i}=\operatorname{diag}\left(\partial g\left(\mathbf{z}_{i 1}^{\top} \gamma\right) / \partial \delta_{i 1}, \ldots, \partial g\left(\mathbf{z}_{i s}^{\top} \gamma\right) / \partial \delta_{i s}\right)$.

Note que $\boldsymbol{\alpha}_{i}=\left(\alpha_{i j}, \ldots, \alpha_{i s}\right)^{\top}$ e temos,

$$
\boldsymbol{\alpha}_{i}=\exp \left(\boldsymbol{\delta}_{i}\right) \quad \text { e } \quad \boldsymbol{\delta}_{i}=\mathbf{Z}_{i} \boldsymbol{\gamma}
$$

em que $\boldsymbol{\gamma}=\left(\gamma_{1}, \ldots \gamma_{q}\right)^{\top}$ é o vetor de parâmetros desconhecidos de dimensão $\mathrm{q} \times 1, \mathbf{Z}_{i}=\left(\mathbf{z}_{i j} \ldots \mathbf{z}_{i l}\right)^{\top}$ é a matriz de covariáveis referente à $i$-ésima unidade experimental, com dimensão $s \times q, \mathbf{G}_{i}=$ $\operatorname{diag}\left(\partial g^{-1}\left(\mathbf{z}_{i 1}^{\top} \gamma\right) / \partial \delta_{i 1}, \ldots, \partial g^{-1}\left(\mathbf{z}_{i s}^{\top} \gamma\right) / \partial \delta_{i s}\right)$ e $\mathbf{M}_{i}=\operatorname{diag}\left(-\frac{2}{\alpha_{i 1}^{2}}, \ldots,-\frac{2}{\alpha_{i s}^{2}}\right)$.

A matriz de variância-covariância de $\mathbf{v}_{i}$ pode ser escrita na forma

$$
\operatorname{Cov}\left(\mathbf{v}_{i}\right)=\operatorname{Var}\left(\mathbf{v}_{i}\right)^{1 / 2} \operatorname{Corr}\left(\mathbf{v}_{i}\right) \operatorname{Var}\left(\mathbf{v}_{i}\right)^{1 / 2},=\Sigma_{i}^{1 / 2} \mathbf{R}\left(\mathbf{v}_{i}\right) \boldsymbol{\Sigma}_{i}^{1 / 2}
$$

em que $\operatorname{Corr}\left(\mathbf{v}_{i}\right)$ representa a matriz de correlação de $\mathbf{v}_{i}$ e $\operatorname{Var}\left(\mathbf{v}_{i}\right)$ é dada por,

$$
\operatorname{Var}\left(\mathbf{v}_{i}\right)=\operatorname{diag}\left\{\operatorname{Var}\left(v_{i j}\right), \ldots, \operatorname{Var}\left(v_{i s}\right)\right\}=\operatorname{diag}\left(\mathrm{E}\left(v_{i 1}^{2}\right), \ldots, \mathrm{E}\left(v_{i s}^{2}\right)\right)=\boldsymbol{\Omega}_{i},
$$

em que,

$$
\begin{aligned}
\operatorname{Var}\left(\mathbf{v}_{i}\right) & =\operatorname{diag}\left\{\operatorname{Var}\left(\mathbf{v}_{i j}\right), \ldots, \operatorname{Var}\left(\mathbf{v}_{i s}\right)\right\} \\
& =\operatorname{diag}\left\{\mathrm{E}\left(\mathbf{v}_{i j}^{2}\right)-\mathrm{E}^{2}\left(\mathbf{v}_{i j}^{2}\right), \ldots, \mathrm{E}\left(\mathbf{v}_{i s}^{2}\right)-\mathrm{E}^{2}\left(\mathbf{v}_{i s}^{2}\right)\right\} \\
& =\operatorname{diag}\left\{\mathrm{E}\left(\mathbf{v}_{i j}^{2}\right), \ldots, \mathrm{E}\left(\mathbf{v}_{i s}^{2}\right)\right\} \\
& =\operatorname{diag}\left\{\frac{2}{\alpha_{i j}^{2}}, \ldots, \frac{2}{\alpha_{i S}^{2}}\right\} .
\end{aligned}
$$

Temos então que a função de estimação linear para $\gamma$ é dada por,

$$
\boldsymbol{\zeta}_{n}=\sum_{i=1}^{n} \mathbf{Z}_{\mathbf{i}}^{\top} \mathbf{G}_{i} \mathbf{M}_{i} \boldsymbol{\Omega}_{i}^{-1 / 2} \mathbf{R}(\rho)^{-1} \boldsymbol{\Omega}_{i}^{-1 / 2} \mathbf{v}_{i},
$$

em que $\mathbf{G}_{i}=\operatorname{diag}\left(\partial g^{-1}\left(\mathbf{z}_{i 1}^{\top} \boldsymbol{\gamma}\right) / \partial \delta_{i 1}, \ldots, \partial g^{-1}\left(\mathbf{z}_{i s}^{\top} \gamma\right) / \partial \delta_{i s}\right)$ e $\mathbf{D}_{i}=\operatorname{diag}\left(\frac{-2}{\alpha_{i j}^{2}}, \ldots, \frac{-2}{\alpha_{i s}^{2}}\right)$.

Temos então que a função de estimação linear para $\gamma$ é dada por,

$$
\boldsymbol{\zeta}_{n}=\sum_{i=1}^{n} \mathbf{Z}_{i}^{\top} \mathbf{G}_{i} \mathbf{M}_{i} \operatorname{Cov}_{\gamma}^{-1}\left(\mathbf{v}_{i}\right) \mathbf{v}_{i}
$$




\section{F.1.2 Derivação da Função de Estimação Conjunta}

Seja $\boldsymbol{\theta}=\left(\boldsymbol{\beta}^{\top}, \boldsymbol{\gamma}^{\top}\right)^{\top}$ e consideremos $\mathbf{d}_{i}=\left(\mathbf{u}_{i}^{\top}, \mathbf{v}_{i}^{\top}\right)^{\top}$. Uma função de estimação ótima para $\boldsymbol{\theta}$ gerada por $\mathbf{d}_{i}$, é dada por,

$$
\boldsymbol{\Gamma}_{n}(\boldsymbol{\theta})^{0}=\mathrm{E} \sum_{i=1}^{n}\left(\begin{array}{cc}
\frac{\partial \mathbf{u}_{i}}{\partial \boldsymbol{\beta}^{\top}} & \frac{\partial \mathbf{u}_{i}}{\partial \boldsymbol{\gamma}^{\top}} \\
\frac{\partial \mathbf{v}_{i}}{\partial \boldsymbol{\beta}^{\top}} & \frac{\partial \mathbf{v}_{i}}{\partial \boldsymbol{\gamma}^{\top}}
\end{array}\right)^{\top} \operatorname{Cov}\left(\mathbf{d}_{i}\right)^{-1} \mathbf{d}_{i} .
$$

Note que,

$$
\begin{aligned}
\operatorname{E}\left\{\operatorname{senh}\left(y_{i j}-\mu_{i j}\right)\right\}= & \int_{-\infty}^{\infty} \operatorname{senh}\left(y_{i j}-\mu_{i j}\right) \frac{1}{\alpha \sqrt{2 \pi}} \cosh \left(\frac{y_{i j}-\mu_{i j}}{2}\right) \\
& \times \exp \left[-2\left\{\frac{1}{\alpha} \operatorname{senh}\left(\frac{y_{i j}-\mu_{i j}}{2}\right)\right\}^{2}\right] d y_{i j} \\
= & \int_{-\infty}^{\infty} \operatorname{senh}\left(\frac{y_{i j}-\mu_{i j}}{2}\right) \cosh \left(\frac{y_{i j}-\mu_{i j}}{2}\right) \frac{1}{\alpha \sqrt{2 \pi}} \\
& \times \cosh \left(\frac{y_{i j}-\mu_{i j}}{2}\right) \exp \left[-2\left\{\frac{1}{\alpha} \operatorname{senh}\left(\frac{y_{i j}-\mu_{i j}}{2}\right)\right\}^{2}\right] d y_{i j} .
\end{aligned}
$$

Fazendo a substituição $a=\frac{y_{i j}-\mu_{i j}}{2}$ e observando que o integrando resultante é uma função ímpar no intervalo $(-\infty, \infty)$, segue que,

$$
\mathrm{E}\left\{\operatorname{senh}\left(y_{i j}-\mu_{i j}\right)\right\}=0
$$

e podemos observar ainda,

$$
\mathrm{E}\left\{\operatorname{senh}\left(\frac{y_{i j}-\mu_{i j}}{2}\right) \cosh \left(\frac{y_{i j}-\mu_{i j}}{2}\right)\right\}=0
$$

Portanto,

$$
\begin{aligned}
\mathrm{E}\left(\frac{\partial \mathbf{u}_{i}}{\partial \boldsymbol{\gamma}^{\top}}\right)^{\top} & =\mathrm{E}\left(\frac{\partial \mathbf{u}_{i}}{\partial \boldsymbol{\alpha}_{i}^{\top}} \frac{\partial \boldsymbol{\alpha}_{i}}{\partial \boldsymbol{\delta}_{i}^{\top}} \frac{\partial \boldsymbol{\delta}_{i}}{\partial \boldsymbol{\gamma}^{\top}}\right)^{\top} \\
& =\mathbf{Z}_{i}^{\top} \mathbf{G}_{i} \operatorname{diag}\left\{-\mathrm{E}\left(\frac{2}{\alpha_{i 1}^{3}} \operatorname{senh}\left(y_{i j}-\mu_{i j}\right)\right), \ldots,-\mathrm{E}\left(\frac{2}{\alpha_{i s}^{3}} \operatorname{senh}\left(y_{i s}-\mu_{i s}\right)\right)\right\} \\
& =\mathbf{0}
\end{aligned}
$$

e

$$
\begin{aligned}
\mathrm{E}\left(\frac{\partial \mathbf{v}_{i}}{\partial \boldsymbol{\beta}^{\top}}\right)^{\top}= & \mathrm{E}\left(\frac{\partial \mathbf{v}_{i}}{\partial \boldsymbol{\beta}^{\top}} \frac{\partial \boldsymbol{\beta}_{i}}{\partial \boldsymbol{\eta}_{i}^{\top}} \frac{\partial \boldsymbol{\eta}_{i}}{\partial \boldsymbol{\beta}^{\top}}\right)^{\top} \\
= & \mathbf{X}_{\mathbf{i}}^{\top} \operatorname{diag}\left\{-\mathrm{E}\left(\frac{4}{\alpha_{i 1}^{3}} \operatorname{senh}\left(\frac{y_{i j}-\mu_{i j}}{2}\right) \cosh \left(\frac{y_{i j}-\mu_{i j}}{2}\right)\right), \ldots,\right. \\
& \left.-\mathrm{E}\left(\frac{4}{\alpha_{i s}^{3}} \operatorname{senh}\left(\frac{y_{i j}-\mu_{i j}}{2}\right) \cosh \left(\frac{y_{i j}-\mu_{i j}}{2}\right)\right)\right\} \\
= & \mathbf{0} .
\end{aligned}
$$


Portanto,

$$
\boldsymbol{\Gamma}_{n}(\boldsymbol{\theta})^{0}=\sum_{i=1}^{n}\left(\begin{array}{cc}
\mathbf{X}_{i}^{\top} \mathbf{N}_{i} & \mathbf{0} \\
\mathbf{0} & \mathbf{Z}_{i}^{\top} \mathbf{G}_{i} \mathbf{M}_{\mathbf{i}}
\end{array}\right) \mathbf{A}_{i}^{-1} \mathbf{d}_{i}
$$

com,

$$
\begin{aligned}
\mathbf{A}_{i}=\operatorname{cov}\left(\mathbf{d}_{i}\right) & =\left(\begin{array}{cc}
\operatorname{Cov}\left(\mathbf{u}_{\mathbf{i}}\right) & \operatorname{Cov}\left(\mathbf{u}_{\mathbf{i}}, \mathbf{v}_{i}\right) \\
\operatorname{Cov}\left(\mathbf{u}_{\mathbf{i}}, \mathbf{v}_{i}\right) & \operatorname{Cov}\left(\mathbf{v}_{i}\right)
\end{array}\right) \\
& =\left(\begin{array}{cc}
\boldsymbol{\Sigma}_{i}^{1 / 2} \operatorname{Corr}\left(\mathbf{u}_{i}\right) \boldsymbol{\Sigma}_{i}^{1 / 2}, & \operatorname{Cov}\left(\mathbf{u}_{\mathbf{i}}, \mathbf{v}_{i}\right) \\
\operatorname{Cov}\left(\mathbf{u}_{\mathbf{i}}, \mathbf{v}_{i}\right) & \boldsymbol{\Omega}_{i}^{1 / 2} \operatorname{Corr}\left(\mathbf{v}_{i}\right) \boldsymbol{\Omega}_{i}^{1 / 2}
\end{array}\right),
\end{aligned}
$$

em que $\mathbf{N}_{i}=\mathrm{E}\left(\frac{\partial \mathbf{u}_{i}}{\partial \boldsymbol{\mu}_{i}}\right)=\operatorname{diag}\left\{\mathrm{E}\left(\dot{u}_{i}\right), \ldots, \mathrm{E}\left(\dot{u}_{i s}\right)\right\}, \operatorname{com} \mathrm{E}\left(\dot{u}_{i j}\right)=\frac{1}{4} \mathrm{E}\left\{\operatorname{sech}^{2}\left(\frac{y_{i 1}-\mu_{i 1}}{2}\right)\right\}-\frac{1}{2}-\frac{1}{\alpha_{i j}^{2}}$, $\operatorname{Corr}\left(\mathbf{u}_{i}\right)$ é a verdadeira matriz de correlação, $\boldsymbol{\Sigma}_{i}=\operatorname{diag}\left\{\operatorname{Var}\left(u_{i 1}\right), \ldots, \operatorname{Var}\left(u_{i s}\right)\right\}, \operatorname{com} \operatorname{Var}\left(u_{i j}\right)=$ $\mathrm{E}\left\{\frac{1}{4} \operatorname{tgh}^{2}\left(\frac{y_{i j}-\mu_{i j}}{2}\right)\right\}+\frac{1}{4}+\frac{1}{\alpha_{i j}^{2}}, \mathbf{G}_{i}=\operatorname{diag}\left(\partial g^{-1}\left(\mathbf{z}_{i 1}^{\top} \gamma\right) / \partial \delta_{i 1}, \ldots, \partial g^{-1}\left(\mathbf{z}_{i s}^{\top} \boldsymbol{\gamma}\right) / \partial \delta_{i s}\right)$,

$\mathbf{M}_{i}=\operatorname{diag}\left(-\frac{2}{\alpha_{i 1}^{2}}, \ldots,-\frac{2}{\alpha_{i s}^{2}}\right), \operatorname{Corr}\left(\mathbf{v}_{i}\right)$ é a verdadeira matriz de correlação e $\boldsymbol{\Omega}_{i}=\operatorname{diag}\left(\frac{2}{\alpha_{i 1}^{2}}, \ldots, \frac{2}{\alpha_{i s}^{2}}\right)$.

\section{F.1.3 Derivação das matrizes de variabilidade e sensibilidade}

Nesta seção vamos derivar a matriz de sensibilidade e a matriz de variabilidade para a função de estimação dada em (F.3). Observe que,

$$
\frac{\partial \boldsymbol{\Gamma}(\boldsymbol{\gamma})}{\partial \boldsymbol{\gamma}}=\frac{\partial \boldsymbol{\Gamma}(\boldsymbol{\alpha})}{\partial \boldsymbol{\alpha}_{i}} \times \frac{\partial \boldsymbol{\alpha}_{i}}{\partial \boldsymbol{\delta}_{i}} \times \frac{\partial \boldsymbol{\delta}_{i}}{\partial \boldsymbol{\gamma}}
$$

Temos que

$$
\begin{aligned}
\frac{\partial \boldsymbol{\Gamma}(\boldsymbol{\gamma})}{\partial \boldsymbol{\gamma}}= & \frac{\partial}{\partial \boldsymbol{\gamma}}\left\{\mathbf{Z}_{\mathbf{i}}^{\top} \mathbf{G}_{i} \mathbf{M}_{i} \boldsymbol{\Omega}_{i}^{-1} \mathbf{v}_{i}\right\} \\
= & \left\{\mathbf{Z}_{\mathbf{i}}^{\top} \frac{\partial \mathbf{G}_{i}}{\partial \boldsymbol{\alpha}_{i}} \mathbf{M}_{i} \boldsymbol{\Omega}_{i}^{-1} \mathbf{v}_{i}+\mathbf{Z}_{\mathbf{i}}^{\top} \mathbf{G}_{i} \frac{\partial \mathbf{M}_{i}}{\partial \boldsymbol{\alpha}_{i}} \boldsymbol{\Omega}_{i}^{-1} \mathbf{v}_{i}+\mathbf{Z}_{\mathbf{i}}^{\top} \mathbf{G}_{i} \mathbf{M}_{i} \frac{\partial \boldsymbol{\Omega}_{i}^{-1}}{\boldsymbol{\alpha}_{i}} \mathbf{v}_{i}\right. \\
& \left.+\mathbf{Z}_{\mathbf{i}}^{\top} \mathbf{G}_{i} \mathbf{M}_{i} \boldsymbol{\Omega}_{i}^{-1} \frac{\partial \mathbf{v}_{i}}{\partial \boldsymbol{\alpha}_{i}}\right\} \mathbf{G}_{i} \mathbf{Z}_{i} .
\end{aligned}
$$

Note que

$$
\begin{gathered}
\frac{\partial \mathrm{E}\left(\dot{v}_{i j}\right)}{\partial \alpha_{i j}}=\frac{4}{\alpha_{i j}^{3}} \quad \text { e } \quad \frac{\partial \mathrm{E}\left(v_{i j}^{2}\right)}{\partial \alpha_{i j}}=-\frac{4}{\alpha_{i j}^{3}}, \\
\frac{\partial \boldsymbol{\Omega}_{i}}{\partial \boldsymbol{\alpha}}=\operatorname{diag}\left(-\frac{4}{\alpha_{i 1}^{3}}, \ldots,-\frac{4}{\alpha_{i s}^{3}}\right),
\end{gathered}
$$

em que,

$$
\mathrm{E}\left\{\mathbf{Z}_{\mathbf{i}}^{\top} \frac{\partial \mathbf{G}_{i}}{\partial \boldsymbol{\alpha}_{i}} \mathbf{M}_{i} \boldsymbol{\Omega}_{i}^{-1} \mathbf{v}_{i} \mathbf{G}_{i} \mathbf{Z}_{i}\right\}=\mathbf{0}
$$




$$
\boldsymbol{\Omega}_{i}^{-1}=\operatorname{diag}\left\{\frac{1}{\mathrm{E}\left(v_{i j}^{2}\right)}, \ldots, \frac{1}{\mathrm{E}\left(v_{i s}^{2}\right)}\right\}=\operatorname{diag}\left\{\frac{\alpha_{i 1}{ }^{2}}{2}, \ldots, \frac{\alpha_{i s}^{2}}{2}\right\} \Rightarrow \frac{\partial \boldsymbol{\Omega}_{i}^{-1}}{\partial \boldsymbol{\alpha}_{i}}=\operatorname{diag}\left\{\alpha_{i 1}, \ldots, \alpha_{i s}\right\},
$$

$\log$,

$$
\mathrm{E}\left\{\mathbf{Z}_{\mathbf{i}}^{\top} \mathbf{G}_{i} \mathbf{M}_{i} \frac{\partial \boldsymbol{\Omega}_{i}^{-1}}{\boldsymbol{\alpha}_{i}} \mathbf{v}_{i} \mathbf{G}_{i} \mathbf{Z}_{i}\right\}=\mathbf{0}
$$

e ainda

$$
\frac{\partial \mathbf{M}_{i}}{\partial \boldsymbol{\alpha}}=\operatorname{diag}\left(\frac{4}{\alpha_{i j}^{3}}, \ldots, \frac{4}{\alpha_{i s}^{3}}\right) \text { e assim, } \mathrm{E}\left\{\mathbf{Z}_{\mathbf{i}}^{\top} \mathbf{G}_{i} \frac{\partial \mathbf{M}_{i}}{\partial \boldsymbol{\alpha}} \boldsymbol{\Omega}_{i}^{-1} \mathbf{v}_{i} \mathbf{G}_{i} \mathbf{Z}_{i}\right\}=\mathbf{0} .
$$

Portanto,

$$
\begin{aligned}
\mathrm{E}\left(\frac{\partial \boldsymbol{\Gamma}(\boldsymbol{\alpha})}{\partial \boldsymbol{\gamma}}\right) \mathrm{E}\left(\mathbf{Z}_{\mathbf{i}}^{\top} \mathbf{G}_{i} \mathbf{M}_{i} \boldsymbol{\Omega}_{i}^{-1 / 2} \mathbf{R}(\rho)^{-1} \boldsymbol{\Omega}_{i}^{-1 / 2} \frac{\partial \mathbf{v}_{i}}{\partial \alpha_{i j}}\right) & \\
& =\mathbf{Z}_{\mathbf{i}}^{\top} \mathbf{G}_{i} \mathbf{M}_{i} \boldsymbol{\Omega}_{i}^{-1} \mathbf{M}_{i} \mathbf{H}_{i} \mathbf{Z}_{i}
\end{aligned}
$$

Do Apêndice B.2 temos que

$$
\mathrm{E}\left(\frac{\partial \boldsymbol{\Gamma}(\boldsymbol{\beta})}{\partial \boldsymbol{\beta}}\right)=\mathrm{E}\left(\frac{\partial \boldsymbol{\psi}_{i}(\boldsymbol{\beta})}{\partial \boldsymbol{\beta}}\right)=\mathbf{X}_{\mathbf{i}}^{\top} \mathbf{N}_{i} \boldsymbol{\Sigma}_{i}^{-1 / 2} \mathbf{R}(\rho)^{-1} \boldsymbol{\Sigma}_{i}^{-1 / 2} \mathbf{N}_{i} \mathbf{X}_{i}
$$

Temos que,

$$
\begin{aligned}
\mathrm{E}\left\{\frac{\partial \boldsymbol{\Gamma}(\boldsymbol{\beta})}{\partial \boldsymbol{\gamma}}\right\}= & \mathrm{E}\left\{\frac{\partial \boldsymbol{\psi}_{i}(\boldsymbol{\beta})}{\partial \boldsymbol{\gamma}}\right\}=\mathrm{E}\left\{\frac{\partial}{\partial \boldsymbol{\gamma}}\left\{\mathbf{X}_{\mathbf{i}}^{\top} \mathbf{N}_{i} \boldsymbol{\Sigma}_{i}^{-1 / 2} \mathbf{R}(\rho)^{-1} \boldsymbol{\Sigma}_{i}^{-1 / 2} \mathbf{u}_{\mathbf{i}}\right\}\right\} \\
= & \mathrm{E}\left\{\left[\mathbf{X}_{\mathbf{i}}^{\top} \frac{\partial \mathbf{N}_{i}}{\partial \boldsymbol{\alpha}_{i}} \boldsymbol{\Sigma}_{i}^{-1 / 2} \mathbf{R}(\rho)^{-1} \boldsymbol{\Sigma}_{i}^{-1 / 2} \mathbf{u}_{\mathbf{i}}+\mathbf{X}_{\mathbf{i}}^{\top} \mathbf{N}_{i} \frac{\partial \boldsymbol{\Sigma}_{i}^{-1 / 2}}{\partial \boldsymbol{\alpha}_{i}} \mathbf{R}(\rho)^{-1} \boldsymbol{\Sigma}_{i}^{-1 / 2} \mathbf{u}_{\mathbf{i}}\right.\right. \\
& \left.\left.+\mathbf{X}_{\mathbf{i}}^{\top} \mathbf{N}_{i} \boldsymbol{\Sigma}_{i}^{-1 / 2} \mathbf{R}(\rho)^{-1} \frac{\partial \boldsymbol{\Sigma}_{i}^{-1 / 2}}{\partial \boldsymbol{\alpha}_{i}} \mathbf{u}_{\mathbf{i}}+\mathbf{X}_{\mathbf{i}}^{\top} \mathbf{N}_{i} \boldsymbol{\Sigma}_{i}^{-1 / 2} \mathbf{R}(\rho)^{-1} \boldsymbol{\Sigma}_{i}^{-1 / 2} \frac{\partial \mathbf{u}_{\mathbf{i}}}{\partial \boldsymbol{\alpha}_{i}}\right] \mathbf{G}_{i} \mathbf{Z}_{i}\right\} \\
= & \mathbf{0}
\end{aligned}
$$

e

$$
\begin{aligned}
\frac{\partial \boldsymbol{\Gamma}(\gamma)}{\partial \boldsymbol{\beta}}= & \frac{\partial}{\partial \boldsymbol{\beta}}\left\{\mathbf{Z}_{\mathbf{i}}^{\top} \mathbf{G}_{i} \mathbf{M}_{i} \boldsymbol{\Omega}_{i}^{-1} \mathbf{v}_{i}\right\} \\
= & \left\{\mathbf{Z}_{\mathbf{i}}^{\top} \frac{\partial \mathbf{G}_{i}}{\partial \boldsymbol{\mu}_{i}} \mathbf{M}_{i} \boldsymbol{\Omega}_{i}^{-1} \mathbf{v}_{i}+\mathbf{Z}_{\mathbf{i}}^{\top} \mathbf{G}_{i} \frac{\partial \mathbf{M}_{i}}{\partial \boldsymbol{\mu}_{i}} \boldsymbol{\Omega}_{i}^{-1} \mathbf{v}_{i}+\mathbf{Z}_{\mathbf{i}}^{\top} \mathbf{G}_{i} \mathbf{M}_{i} \frac{\partial \boldsymbol{\Omega}_{i}^{-1}}{\boldsymbol{\mu}_{i}} \mathbf{v}_{i}\right. \\
& \left.+\mathbf{Z}_{\mathbf{i}}^{\top} \mathbf{G}_{i} \mathbf{M}_{i} \boldsymbol{\Omega}_{i}^{-1} \frac{\partial \mathbf{v}_{i}}{\partial \boldsymbol{\mu}_{i}}\right\} \mathbf{G}_{i} \mathbf{Z}_{i} \\
= & \mathbf{0}
\end{aligned}
$$

A matriz de sensibilidade e a matriz de variabilidade para a equação de estimação (F.1) são, 
respectivamente, dadas por

$$
\begin{aligned}
\mathbf{S}_{\mathbf{i}}(\boldsymbol{\theta}) & =\mathrm{E}\left(\begin{array}{cc}
\frac{\partial}{\partial \boldsymbol{\beta}^{\top}} \mathbf{\Psi}_{i}(\boldsymbol{\beta}) & \frac{\partial}{\partial \boldsymbol{\gamma}^{\top}} \mathbf{\Psi}_{i}(\boldsymbol{\beta}) \\
\frac{\partial}{\partial \boldsymbol{\beta}^{\top}} \boldsymbol{\zeta}_{i}(\boldsymbol{\gamma}) & \frac{\partial}{\partial \boldsymbol{\gamma}^{\top}} \boldsymbol{\zeta}_{i}(\boldsymbol{\gamma})
\end{array}\right) \\
& =\left(\begin{array}{cc}
\mathbf{X}_{\mathbf{i}}^{\top} \mathbf{N}_{i} \boldsymbol{\Sigma}_{i}^{-1 / 2} \mathbf{R}(\rho)^{-1} \boldsymbol{\Sigma}_{i}^{-1 / 2} \mathbf{N}_{i} \mathbf{X}_{i} . & \mathbf{0} \\
\mathbf{0} & \mathbf{Z}_{\mathbf{i}}^{\top} \mathbf{G}_{i} \mathbf{M}_{i} \boldsymbol{\Omega}_{i}^{-1} \mathbf{M}_{i} \mathbf{G}_{i} \mathbf{Z}_{i} .
\end{array}\right) \\
& =\mathbf{Q}_{\mathbf{i}}^{\top} \boldsymbol{\Lambda}_{i} \mathbf{\Upsilon}_{i}^{-1} \boldsymbol{\Lambda}_{i} \mathbf{Q}_{\mathbf{i}},
\end{aligned}
$$

$\log 0$

$$
\sum_{i=1}^{n} \mathbf{S}_{\mathbf{i}}(\boldsymbol{\theta})=\sum_{i=1}^{n} \mathrm{E}\left\{\mathbf{Q}_{\mathbf{i}}^{\top} \boldsymbol{\Lambda}_{i} \boldsymbol{\Upsilon}_{i}^{-1} \mathbf{d}_{i} \mathbf{d}_{i}^{\top}\right\}=\sum_{i=1}^{n} \mathbf{Q}_{\mathbf{i}}^{\top} \boldsymbol{\Lambda}_{i} \boldsymbol{\Upsilon}_{i}^{-1} \mathrm{E}\left\{\mathbf{d}_{i} \mathbf{d}_{i}^{\top}\right\} \boldsymbol{\Upsilon}_{i}^{-\top} \boldsymbol{\Lambda}_{i}^{\top} \mathbf{Q}_{\mathbf{i}}
$$

Temos ainda que,

$$
\begin{aligned}
\mathbf{V}_{i}(\boldsymbol{\theta}) & =\mathrm{E}\left[\mathbf{Q}_{\mathbf{i}}^{\top} \boldsymbol{\Lambda}_{i} \mathbf{\Upsilon}_{i}^{-1} \mathbf{d}_{i} \mathbf{d}_{i}^{\top} \mathbf{\Upsilon}_{i}^{-\top} \boldsymbol{\Lambda}_{i} \mathbf{Q}_{\mathbf{i}}\right] \\
& =\mathbf{Q}_{\mathbf{i}}^{\top} \boldsymbol{\Lambda}_{i} \mathbf{\Upsilon}_{i}^{-1} \mathrm{E}\left[\mathbf{d}_{i} \mathbf{d}_{i}^{\top}\right] \mathbf{\Upsilon}_{i}^{-\top} \boldsymbol{\Lambda}_{i} \mathbf{Q}_{\mathbf{i}} \\
& =\mathbf{Q}_{\mathbf{i}}^{\top} \boldsymbol{\Lambda}_{i} \mathbf{\Upsilon}_{i}^{-1} \operatorname{Cov}\left(\mathbf{d}_{i}\right) \mathbf{\Upsilon}_{i}^{-\top} \boldsymbol{\Lambda}_{i} \mathbf{Q}_{\mathbf{i}}
\end{aligned}
$$

\section{F.1.4 Influência conformal sob heterogeneidade do parâmetro de forma}

Perturbação da variável resposta

Observe que

$$
v_{\omega i j}=\frac{1}{\alpha_{i j}}\left[\frac{4}{\alpha_{i j}^{2}} \operatorname{senh}^{2}\left(\frac{y_{\omega i j}-\mu_{i j}}{2}\right)-1\right],
$$

cuja derivada em relação a $\omega_{i j}$ é dada por,

$$
\begin{aligned}
\frac{\partial v_{\omega_{i j}}}{\partial \omega_{i j}} & =\frac{4}{\alpha_{i j}^{3}} \operatorname{senh}\left(\frac{y_{\omega i j}-\mu_{i j}}{2}\right) \cosh \left(\frac{y_{i j}-\mu_{i j}}{2}\right) \frac{\partial \alpha_{\omega i j}}{\partial \omega_{i j}} \\
& =\frac{2 s_{y_{i j}}}{\alpha^{3}} \operatorname{senh}\left(y_{\omega i j}-\mu_{i j}\right) .
\end{aligned}
$$

\section{Perturbação individual das covariáveis}

\section{Matrizes X e Z iguais}

Nesta Seção obtemos derivadas de $\boldsymbol{\Psi}_{\omega i}, \boldsymbol{\Lambda}_{\omega i}$ e $\mathbf{d}_{\omega i}$ com relação à $\boldsymbol{\omega}_{i}^{\top}$.

$$
\boldsymbol{\Lambda}_{\omega i}=\left(\begin{array}{cc}
\mathbf{N}_{\omega i} & \mathbf{0} \\
\mathbf{0} & \mathbf{G}_{\omega i} \mathbf{M}_{\omega i}
\end{array}\right), \quad \Upsilon_{\omega i}=\left(\begin{array}{cc}
\boldsymbol{\Sigma}_{\omega i}^{1 / 2} \mathbf{R}(\rho) \boldsymbol{\Sigma}_{\omega i}^{1 / 2} & \mathbf{0} \\
\mathbf{0} & \boldsymbol{\Omega}_{\omega i}
\end{array}\right) \text { e } \mathbf{d}_{\omega i}=\left(\begin{array}{c}
\mathbf{u}_{\omega i} \\
\mathbf{v}_{\omega i}
\end{array}\right)
$$

em que $\mathbf{N}_{\omega i}=\operatorname{diag}\left\{\mathrm{E}\left(\dot{u}_{\omega i 1}\right), \ldots, \mathrm{E}\left(\dot{u}_{\omega i s}\right)\right\}, \operatorname{com} \mathrm{E}\left(\dot{u}_{\omega i j}\right)=\frac{1}{4} \mathrm{E}\left\{\operatorname{sech}^{2}\left(\frac{y_{i j}-\mu_{\omega i j}}{2}\right)\right\}-\frac{1}{2}-\frac{1}{\alpha_{\omega i j}^{2}}, \boldsymbol{\Sigma}_{\omega i}=$ $\operatorname{diag}\left\{\operatorname{Var}\left(u_{\omega i 1}\right), \ldots, \operatorname{Var}\left(u_{\omega i s}\right)\right\} \quad\left(\Sigma_{\omega i}^{1 / 2}=\operatorname{diag}\left\{\operatorname{Var}^{1 / 2}\left(u_{\omega i 1}\right), \ldots, \operatorname{Var}^{1 / 2}\left(u_{\omega i s}\right)\right\}\right), \operatorname{com} \operatorname{Var}\left(u_{\omega i j}\right)=$ 
$\mathrm{E}\left\{\frac{1}{4} \operatorname{tgh}^{2}\left(\frac{y_{i j}-\mu_{\omega i j}}{2}\right)\right\}+\frac{1}{4}+\frac{1}{\alpha_{\omega i j}^{2}}, \mathbf{G}_{i}=\operatorname{diag}\left(\partial g\left(\mathbf{z}_{i 1}^{\top} \gamma\right) / \partial \delta_{i 1}, \ldots, \partial g\left(\mathbf{z}_{i s}^{\top} \gamma\right) / \partial \delta_{i s}\right), \mathbf{M}_{\omega i}=$ $\operatorname{diag}\left(-\frac{2}{\alpha_{\omega i j}^{2}}, \ldots,-\frac{2}{\alpha_{\omega i s}^{2}}\right), \boldsymbol{\Omega}_{\omega i}=\operatorname{diag}\left(\frac{2}{\alpha_{\omega i 1}^{2}}, \ldots, \frac{2}{\alpha_{\omega i s}^{2}}\right), u_{\omega i j}=-\frac{1}{2} \operatorname{tgh}\left(\frac{y_{i j}-\mu_{\omega i j}}{2}\right)+\frac{1}{\alpha_{\omega i j}^{2}} \operatorname{senh}\left(y_{i j}-\right.$ $\left.\mu_{\omega i j}\right)$ e $v_{i j}=\frac{1}{\alpha_{\omega i j}}\left[\frac{4}{\alpha_{\omega i j}^{2}} \operatorname{senh}^{2}\left(\frac{y_{i j}-\mu_{\omega i j}}{2}\right)-1\right]$.

Temos que,

$$
\begin{aligned}
\frac{\partial \boldsymbol{\Lambda}_{\omega i}}{\partial \boldsymbol{\omega}_{i}^{\top}} & =\left(\begin{array}{cc}
2 \mathbf{G}_{\omega i} \dot{\mathbf{M}}_{\omega i} \gamma_{k} s_{x k} & \mathbf{0} \\
\mathbf{0} & \dot{\mathbf{G}}_{\omega i} \mathbf{M}_{\omega i}+\mathbf{G}_{\omega i} \dot{\mathbf{M}}_{\omega i}
\end{array}\right), \\
\frac{\partial \mathbf{\Upsilon}_{\omega i}}{\partial \boldsymbol{\omega}_{i}^{\top}} & =\left(\begin{array}{cc}
-\frac{1}{4} \gamma_{k} s_{x k}\left[\boldsymbol{\Sigma}_{\omega i}^{1 / 2} \mathbf{R}(\rho) \Sigma_{\omega i}^{-1 / 2} \dot{\mathbf{M}}_{\omega i} \mathbf{G}_{\omega i}+\Sigma_{\omega i}^{-1 / 2} \dot{\mathbf{M}}_{\omega i} \mathbf{G}_{\omega i} \mathbf{R}(\rho) \Sigma_{\omega i}^{1 / 2}\right] & \mathbf{0} \\
\mathbf{0} & \gamma_{k} s_{x_{k}} \dot{\boldsymbol{\Omega}},
\end{array}\right), \\
\frac{\partial \mathbf{d}_{\omega i}}{\partial \boldsymbol{\omega}_{i}^{\top}} & =\left(\begin{array}{c}
\beta_{k} s_{x k} \mathcal{A}_{\omega i}-\mathcal{B}_{\omega i} \mathbf{G}_{\omega i} \gamma_{k} s_{x_{k}} \\
\mathcal{C}_{\omega i} \mathbf{G}_{\omega i} \gamma_{k} s_{x_{k}}-2 \mathbf{M}_{\omega i} \mathbf{G}_{\omega i} \gamma_{k} s_{x_{k}}-\mathcal{B}_{\omega i} \beta_{k} s_{x k}
\end{array}\right) .
\end{aligned}
$$

em que $\frac{\partial \mathbf{G}_{\omega}}{\partial \boldsymbol{\omega}^{\top}}=\operatorname{diag}\left(\dot{\mathbf{G}}_{\omega 1}, \ldots, \dot{\mathbf{G}}_{\omega s}\right) \mathrm{e} \frac{\partial \mathbf{M}_{\omega}}{\partial \boldsymbol{\omega}^{\top}}=\operatorname{diag}\left(\dot{\mathbf{M}}_{\omega 1}, \ldots, \dot{\mathbf{M}}_{\omega s}\right) \operatorname{com} \dot{\mathbf{M}}_{\omega i}=\operatorname{diag}\left(\frac{4}{\alpha_{\omega i j}^{3}}, \ldots, \frac{4}{\alpha_{\omega i s}^{3}}\right)$ $\dot{\mathbf{G}}_{\omega i}=\operatorname{diag}\left(\partial^{2} g^{-1}\left(\mathbf{z}_{i 1}^{\top} \boldsymbol{\gamma}\right) / \partial \delta_{i 1}^{2}, \ldots, \partial g^{-1}\left(\mathbf{z}_{i s}^{\top} \gamma\right) / \partial \delta_{i s}\right)$ e $\dot{\boldsymbol{\Omega}}_{\omega i}=\operatorname{diag}\left(-\frac{4}{\alpha_{\omega i j}^{3}}, \ldots,-\frac{4}{\alpha_{\omega i s}^{3}}\right)$. Observe ainda que,

$$
\frac{\partial\left[\boldsymbol{\Sigma} \omega i^{1 / 2} \mathbf{R}(\rho) \boldsymbol{\Sigma} \omega i^{1 / 2}\right]}{\partial \boldsymbol{\omega}_{i}^{\top}}=\frac{\partial \boldsymbol{\Sigma}_{\omega i}^{1 / 2}}{\partial \boldsymbol{\omega}_{i}^{\top}} \mathbf{R}(\rho) \boldsymbol{\Sigma} \omega i^{1 / 2}+\boldsymbol{\Sigma}_{\omega}^{1 / 2} \mathbf{R} \frac{\partial \boldsymbol{\Sigma}_{\omega}^{1 / 2}}{\partial \boldsymbol{\omega}_{i}^{\top}} .
$$

E temos que,

$$
\begin{aligned}
\frac{\partial \alpha_{\omega i j}}{\partial \omega_{i j}} & =\frac{\partial g^{-1}\left(\delta_{\omega i j}\right)}{\partial \delta_{i j}} \gamma_{k} s_{x_{k}}, \quad \frac{\partial}{\partial \omega_{i j}}\left[\frac{\partial g^{-1}\left(\delta_{\omega i j}\right)}{\partial \delta_{\omega i j}}\right]=\frac{\partial^{2} g^{-1}\left(\delta_{\omega i j}\right)}{\partial \delta_{\omega i j}^{2}} \gamma_{k} s_{x k} \\
\frac{\partial \mathrm{E}\left(\dot{u}_{\omega i j}\right)}{\partial \omega_{i j}} & =\frac{\partial \frac{1}{4} \mathrm{E}\left\{\operatorname{sech}^{2}\left(\frac{y_{i j}-\mu_{\omega i j}}{2}\right)\right\}}{\partial \mu_{\omega i j}} \frac{\partial \mu_{\omega i j}}{\partial \omega_{i j}}+\frac{\partial 1 / \alpha_{\omega i j}^{2}}{\partial \omega_{i j}}=-\frac{2}{\alpha_{\omega i j}^{3}} \frac{\partial g^{-1}\left(\delta_{\omega i j}\right)}{\partial \delta_{i j}} \frac{\partial \delta_{\omega i j}}{\partial \omega_{i j}} \\
& =-\frac{2}{\alpha_{\omega i j}^{3}} \frac{\partial g^{-1}\left(\delta_{\omega i j}\right)}{\partial \delta_{i j}} \gamma_{k} s_{x_{k}}, \\
\frac{\partial \operatorname{Var}\left(u_{\omega i j}\right)}{\partial \omega_{i j}} & =\frac{\partial \frac{1}{4} \mathrm{E}\left\{\frac{1}{4} \operatorname{tgh}^{2}\left(\frac{y_{i j}-\mu_{\omega i j}}{2}\right)\right\}}{\partial \mu_{\omega i j}} \frac{\partial \mu_{\omega i j}}{\partial \omega_{i j}}-\frac{\partial\left(1 / \alpha_{\omega i j}^{2}\right)}{\partial \omega_{i j}}=\frac{2}{\alpha_{\omega i j}^{3}} \frac{\partial g^{-1}\left(\delta_{\omega i j}\right)}{\partial \delta_{i j}} \frac{\partial \delta_{\omega i j}}{\partial \omega_{i j}} \\
& =\frac{2}{\alpha_{\omega i j}^{3}} \frac{\partial g^{-1}\left(\delta_{\omega i j}\right)}{\partial \delta_{i j}} \gamma_{k} s_{x_{k}},
\end{aligned}
$$$$
\frac{\partial \operatorname{Var}^{1 / 2}\left(u_{\omega i j}\right)}{\partial \omega_{i} j}=\frac{1}{2} \operatorname{Var}^{-1 / 2}\left(u_{\omega i j}\right) \frac{\partial \operatorname{Var}\left(u_{\omega i j}\right)}{\partial \omega_{i j}}
$$

$\frac{\partial u_{i j}}{\partial \omega_{i j}}=\frac{1}{4} \operatorname{sech}^{2}\left(\frac{y_{i j}-\mu_{\omega i j}}{2}\right) \frac{\partial \mu_{\omega i j}}{\partial \omega i j}-\frac{2}{\alpha_{\omega i j}^{3}} \frac{\partial \alpha_{\omega i j}}{\partial \omega i j} \operatorname{senh}\left(y_{i j}-\mu_{\omega i j}\right)-\frac{1}{\alpha_{\omega i j}^{2}} \cosh \left(y_{i j}-\mu_{\omega i j}\right) \frac{\partial \mu_{\omega i j}}{\partial \omega i j}$ 


$$
\begin{aligned}
& =\beta_{k} s_{x k}\left[\frac{1}{4} \operatorname{sech}^{2}\left(\frac{y_{i j}-\mu_{\omega i j}}{2}\right)-\frac{1}{\alpha_{\omega i j}^{2}} \cosh \left(y_{i j}-\mu_{\omega i j}\right)\right]-\frac{2}{\alpha_{\omega i j}^{3}} \operatorname{senh}\left(y_{i j}-\mu_{\omega i j}\right) \frac{\partial g^{-1}\left(\delta_{\omega i j}\right)}{\partial \delta_{i j}} \gamma_{k} s_{x_{k}} \\
& =\beta_{k} s_{x k} \mathcal{A}_{\omega i j}-\mathcal{B}_{\omega i j} \frac{\partial g^{-1}\left(\delta_{\omega i j}\right)}{\partial \delta_{i j}} \gamma_{k} s_{x_{k}}
\end{aligned}
$$

$\mathrm{e}$

$$
\begin{aligned}
\frac{\partial v_{i j}}{\partial \omega_{i j}}= & -\frac{1}{\alpha_{i j}^{2}} \frac{\partial \alpha_{\omega i j}}{\partial \omega_{i j}}\left[\frac{4}{\alpha_{\omega i j}^{2}} \operatorname{senh}^{2}\left(\frac{y_{i j}-\mu_{\omega i j}}{2}\right)-1\right]+\frac{1}{\alpha_{\omega i j}}\left[-\frac{8}{\alpha_{\omega i j}^{3}} \frac{\partial \alpha_{\omega i j}}{\partial \omega_{i j}} \operatorname{senh}^{2}\left(\frac{y_{i j}-\mu_{\omega i j}}{2}\right)\right. \\
& \left.-\frac{2}{\alpha_{\omega i j}^{2}} 2 \operatorname{senh}\left(\frac{y_{i j}-\mu_{\omega i j}}{2}\right) \cosh \left(\frac{y_{i j}-\mu_{\omega i j}}{2}\right) \frac{\partial \mu_{\omega i j}}{\partial \omega_{i j}}\right] \\
= & -\frac{12}{\alpha_{i j}^{4}} \frac{\partial \alpha_{\omega i j}}{\partial \omega_{i j}} \operatorname{senh}^{2}\left(\frac{y_{i j}-\mu_{\omega i j}}{2}\right)+\frac{1}{\alpha_{i j}^{2}} \frac{\partial \alpha_{\omega i j}}{\partial \omega_{i j}}-\frac{2}{\alpha_{\omega i j}^{3}} \operatorname{senh}\left(y_{i j}-\mu_{\omega i j}\right) \frac{\partial \mu_{\omega i j}}{\partial \omega_{i j}} \\
= & -\frac{12}{\alpha_{i j}^{4}} \operatorname{senh}^{2}\left(\frac{y_{i j}-\mu_{\omega i j}}{2}\right) \frac{\partial g^{-1}\left(\delta_{\omega i j}\right)}{\partial \delta_{i j}} \gamma_{k} s_{x_{k}}-2 M_{\omega i j} \frac{\partial g^{-1}\left(\delta_{\omega i j}\right)}{\partial \delta_{i j}} \gamma_{k} s_{x_{k}}-\mathcal{B}_{\omega i j} \beta_{k} s_{x k} \\
= & \mathcal{C}_{\omega i j} \frac{\partial g^{-1}\left(\delta_{\omega i j}\right)}{\partial \delta_{i j}} \gamma_{k} s_{x_{k}}-2 M_{\omega i j} \frac{\partial g^{-1}\left(\delta_{\omega i j}\right)}{\partial \delta_{i j}} \gamma_{k} s_{x_{k}}-\mathcal{B}_{\omega i j} \beta_{k} s_{x k} .
\end{aligned}
$$

\section{Matrizes X e Z totalmente diferentes}

Obteremos agora derivadas de $\boldsymbol{\Psi}_{\omega i}, \boldsymbol{\Lambda}_{\omega i}$ e $\mathbf{d}_{\omega i}$ com relação à $\boldsymbol{\omega}_{i}^{\top}$ quando consideramos a perturbação da $k$-ésima coluna da matriz X e as matrizes para modelar os parâmetros de localização e forma são completamente diferentes.

$$
\boldsymbol{\Lambda}_{\omega i}=\left(\begin{array}{cc}
\mathbf{N}_{\omega i} & \mathbf{0} \\
\mathbf{0} & \mathbf{G}_{i} \mathbf{M}_{i}
\end{array}\right), \quad \Upsilon_{\omega i}=\left(\begin{array}{cc}
\boldsymbol{\Sigma}_{\omega i}^{1 / 2} \mathbf{R}(\rho) \boldsymbol{\Sigma}_{\omega i}^{1 / 2} & \mathbf{0} \\
\mathbf{0} & \boldsymbol{\Omega}_{i}
\end{array}\right) \text { e } \mathbf{d}_{\omega i}=\left(\begin{array}{c}
\mathbf{u}_{\omega i} \\
\mathbf{v}_{\omega i}
\end{array}\right)
$$

em que $\mathbf{N}_{\omega i}=\operatorname{diag}\left\{\mathrm{E}\left(\dot{u}_{\omega i 1}\right), \ldots, \mathrm{E}\left(\dot{u}_{\omega i s}\right)\right\}, \operatorname{com} \mathrm{E}\left(\dot{u}_{\omega i j}\right)=\frac{1}{4} \mathrm{E}\left\{\operatorname{sech}^{2}\left(\frac{y_{i j}-\mu_{\omega i j}}{2}\right)\right\}-\frac{1}{2}-\frac{1}{\alpha_{i j}^{2}}, \boldsymbol{\Sigma}_{\omega i}=$ $\operatorname{diag}\left\{\operatorname{Var}\left(u_{\omega i 1}\right), \ldots, \operatorname{Var}\left(u_{\omega i s}\right)\right\} \quad\left(\Sigma_{\omega i}^{1 / 2}=\operatorname{diag}\left\{\operatorname{Var}^{1 / 2}\left(u_{\omega i 1}\right), \ldots, \operatorname{Var}^{1 / 2}\left(u_{\omega i s}\right)\right\}\right), \operatorname{com} \operatorname{Var}\left(u_{\omega i j}\right)=$ $\mathrm{E}\left\{\frac{1}{4} \operatorname{tgh}^{2}\left(\frac{y_{i j}-\mu_{\omega i j}}{2}\right)\right\}+\frac{1}{4}+\frac{1}{\alpha_{i j}^{2}}, \mathbf{G}_{i}=\operatorname{diag}\left(\partial g\left(\delta_{i 1}\right) / \partial \delta_{i 1}, \ldots, \partial g\left(\delta_{i 1}\right) / \partial \delta_{i s}\right), \mathbf{M}_{i}=\operatorname{diag}\left(-\frac{2}{\alpha_{i 1}^{2}}, \ldots,-\frac{2}{\alpha_{i s}^{2}}\right)$, $\boldsymbol{\Omega}_{i}=\operatorname{diag}\left(\frac{2}{\alpha_{i 1}^{2}}, \ldots, \frac{2}{\alpha_{i s}^{2}}\right), u_{\omega i j}=-\frac{1}{2} \operatorname{tgh}\left(\frac{y_{i j}-\mu_{\omega i j}}{2}\right)+\frac{1}{\alpha_{i j}^{2}} \operatorname{senh}\left(y_{i j}-\mu_{\omega i j}\right)$ e $v_{i j}=\frac{1}{\alpha_{i j}}\left[\frac{4}{\alpha_{i j}^{2}} \operatorname{senh}^{2}\left(\frac{y_{i j}-\mu_{\omega i j}}{2}\right)-1\right]$.

Temos que,

$$
\frac{\partial\left[\boldsymbol{\Sigma} \omega i^{1 / 2} \mathbf{R}(\rho) \boldsymbol{\Sigma}_{\omega i}^{1 / 2}\right]}{\partial \boldsymbol{\omega}_{i}^{\top}}=\frac{\partial \boldsymbol{\Sigma}_{\omega i}^{1 / 2}}{\partial \boldsymbol{\omega}_{i}^{\top}} \mathbf{R}(\rho) \boldsymbol{\Sigma} \omega i^{1 / 2}+\boldsymbol{\Sigma}_{\omega i}^{1 / 2} \mathbf{R} \frac{\partial \boldsymbol{\Sigma}_{\omega}^{1 / 2}}{\partial \boldsymbol{\omega}_{i}^{\top}}
$$

e

$$
\frac{\partial \operatorname{Var}^{1 / 2}\left(u_{\omega i j}\right)}{\partial \omega_{i j}}=\frac{1}{2} \operatorname{Var}^{-1 / 2}\left(u_{\omega i j}\right) \frac{\partial \operatorname{Var}\left(u_{\omega i j}\right)}{\partial \omega_{i j}},
$$

e de forma similar ao que foi feito na Seção B.1 obtemos,

$$
\frac{\partial \operatorname{Var}\left(u_{\omega i j}\right)}{\partial \omega_{i j}}=\frac{\partial \frac{1}{4} \mathrm{E}\left\{\operatorname{tgh}^{2}\left(\frac{y_{i j}-\mu_{\omega i j}}{2}\right)\right\}}{\partial \mu_{\omega i j}} \frac{\partial \mu_{\omega i j}}{\partial \omega_{i j}}=0 \text { e } \frac{\partial \mathrm{E}\left(\dot{u}_{\omega i j}\right)}{\partial \omega_{i j}}=\frac{\partial \frac{1}{4} \mathrm{E}\left\{\operatorname{sech}^{2}\left(\frac{y_{i j}-\mu_{\omega i j}}{2}\right)\right\}}{\partial \mu_{\omega i j}} \frac{\partial \mu_{\omega i j}}{\partial \omega_{i j}}=0 .
$$


Portanto,

$$
\frac{\partial \mathbf{N}_{\omega i}}{\partial \boldsymbol{\omega}_{i}^{\top}}=\frac{\partial \boldsymbol{\Sigma}_{\omega i}}{\partial \boldsymbol{\omega}_{i}^{\top}}=\mathbf{0}_{N s \times N s},
$$

donde

$$
\frac{\partial \boldsymbol{\Lambda}_{\omega i}}{\partial \boldsymbol{\omega}_{i}^{\top}}=\frac{\partial \boldsymbol{\Upsilon}_{\omega i}}{\partial \boldsymbol{\omega}_{i}^{\top}}=\left(\begin{array}{cc}
\mathbf{0} & \mathbf{0} \\
\mathbf{0} & \mathbf{0}
\end{array}\right)
$$

Por outro lado,

$$
\begin{aligned}
\frac{\partial u_{\omega i j}}{\partial \omega i j} & =\left[\frac{1}{4} \operatorname{sech}^{2}\left(\frac{y_{i j}-\mu_{\omega i j}}{2}\right)-\frac{1}{\alpha^{2}} \cosh \left(y_{i j}-\mu_{\omega i j}\right)\right] \frac{\partial \mu_{\omega i j}}{\partial \omega i j} \\
& =\left[\frac{1}{4} \operatorname{sech}^{2}\left(\frac{y_{i j}-\mu_{\omega i j}}{2}\right)-\frac{1}{\alpha^{2}} \cosh \left(y_{i j}-\mu_{\omega i j}\right)\right] \beta_{k} s_{x k}=\beta_{k} s_{x k} \mathcal{A}_{\omega i j}^{*}
\end{aligned}
$$

e

$$
\begin{aligned}
\frac{\partial v_{\omega i j}}{\partial \omega_{i j}} & =\frac{4}{\alpha_{i j}^{3}} 2 \operatorname{senh}\left(\frac{y_{i j}-\mu_{\omega i j}}{2}\right) \cosh \left(\frac{y_{i j}-\mu_{\omega i j}}{2}\right) \times\left(-\frac{1}{2}\right) \frac{\partial \mu_{\omega i j}}{\omega_{i j}} \\
& =-\frac{2}{\alpha_{i j}^{3}} \operatorname{senh}\left(y_{i j}-\mu_{\omega i j}\right) \beta_{k} s_{x k}=\beta_{k} s_{x k} \mathcal{B}_{\omega i j}^{*} .
\end{aligned}
$$

De forma que,

$$
\frac{\partial \mathbf{d}_{\omega i}}{\partial \boldsymbol{\omega}_{i}^{\top}}=\left(\begin{array}{c}
\beta_{k} s_{x k} \mathcal{A}_{\omega i}^{*} \\
-\mathcal{B}_{\omega i}^{*} \beta_{k} s_{x k}
\end{array}\right)
$$

\section{F.2 Função de estimação conjunta para o modelo heterogêneo log- BS-t}

\section{F.2.1 Derivação das matrizes de variabilidade e sensibilidade}

Nesta seção vamos derivar a matriz de sensibilidade e a matriz de variabilidade para a equação de estimação (4.19). Observe que,

$$
\begin{aligned}
\frac{\partial \boldsymbol{\zeta}_{i}(\gamma)}{\partial \boldsymbol{\gamma}}= & \frac{\partial}{\partial \boldsymbol{\gamma}}\left\{\mathbf{Z}_{\mathbf{i}}^{\mathbf{T}} \mathbf{H}_{i} \mathbf{M}_{i} \boldsymbol{\Omega}_{i}^{-1} \mathbf{v}_{i}\right\} \\
= & \left\{\mathbf{Z}_{\mathbf{i}}^{\mathbf{T}} \frac{\partial \mathbf{H}_{i}}{\partial \boldsymbol{\alpha}} \mathbf{M}_{i} \boldsymbol{\Omega}_{i}^{-1 / 2} \mathbf{R}(\rho)^{-1} \boldsymbol{\Omega}_{i}^{-1 / 2} \mathbf{v}_{i}+\mathbf{Z}_{\mathbf{i}}^{\mathbf{T}} \mathbf{H}_{i} \frac{\partial \mathbf{M}_{i}}{\partial \boldsymbol{\alpha}} \boldsymbol{\Omega}_{i}^{-1} \mathbf{v}_{i}\right. \\
& \left.+\mathbf{Z}_{\mathbf{i}}^{\mathbf{T}} \mathbf{H}_{i} \mathbf{M}_{i} \boldsymbol{\Omega}_{i}^{-1} \frac{\partial \mathbf{v}_{i}}{\partial \boldsymbol{\alpha}}\right\} \mathbf{H}_{\mathbf{i}} \mathbf{Z}_{i} .
\end{aligned}
$$

Note que

$$
\mathrm{E}\left(\dot{v}_{i j}\right)=\int_{-\infty}^{\infty}\left\{\frac { 2 } { \alpha } \mathrm { c } ( \nu ) \{ 1 + \frac { 4 } { \nu } ( \frac { x _ { i j } } { \alpha } ) ^ { 2 } \} ^ { - ( \frac { \nu + 1 } { 2 } ) } \left[\frac{1}{\alpha_{i j}^{2}}+\frac{4 w_{i j} x_{i j}^{2}}{\alpha_{i j}^{4}}\left\{-1+\frac{8 x_{i j}^{2}}{\nu \alpha_{i j}^{2}+4 x_{i j}^{2}}\right.\right.\right.
$$




$$
\left.\left.\left.-2 \frac{x_{i j}^{2}}{\alpha_{i j}}\right\}\right]\right\} \mathrm{d} x_{i j}
$$

Temos que,

$$
\frac{d \xi_{i j 1}}{d \alpha_{i j}}=\frac{-1}{\alpha_{i j}} \xi_{i j 1}, \frac{d \xi_{i j 2}}{d \alpha_{i j}}=\frac{-1}{\alpha_{i j}} \xi_{i j 2}
$$

$\mathrm{e}$

$$
\frac{d w_{i j}}{d \alpha_{i j}}=\frac{8 w_{i j} \xi_{i j 2}^{2}}{\alpha_{i j}\left(\nu+4 \xi_{i j 2}^{2}\right)}
$$

Então

$$
\frac{d}{d \alpha_{i j}}\left\{\frac{2}{\alpha} \mathrm{c}(\nu)\left\{1+\frac{4}{\nu}\left(\frac{x_{i j}}{\alpha}\right)^{2}\right\}^{-\left(\frac{\nu+1}{2}\right)}\left[\frac{1}{\alpha_{i j}^{2}}+\frac{4 w_{i j} x_{i j}^{2}}{\alpha_{i j}^{4}}\left(-1+\frac{8 x_{i j}^{2}}{\nu \alpha_{i j}^{2}+4 x_{i j}^{2}}-2 \frac{x_{i j}^{2}}{\alpha_{i j}}\right)\right]\right\} .
$$

Podemos provar facilmente que $\left\{\mathrm{c}(\nu) \xi_{i j 1}\left\{1+\frac{4 \xi_{i j 2}^{2}}{\nu}\right\}^{-\left(\frac{\nu+1}{2}\right)}\left[\frac{1}{4 \alpha^{2} \xi_{i j 1}^{2}}-w_{i j}\left(\xi_{i j 1}^{2}+\xi_{i j 2}^{2}\right)+\frac{w_{i j} 8 \xi_{i j 2}^{2} \xi_{i j 1}^{2}}{\left(\nu+4 \xi_{i j 2}^{2}\right)}\right]\right\}$ é contínua, logo, podemos derivar sob o sinal de integração e fazendo as modificações de variáveis adequadas temos que,

$$
\begin{aligned}
\frac{\partial \mathrm{E}\left\{\dot{u}_{i j}\left(y_{i j}, \mu_{i j}\right)\right\}}{\partial \mu_{i j}}= & \int_{-\infty}^{\infty}\left\{\mathrm { c } ( \nu ) \left\{-\frac{\xi_{i j 2}}{2}\left\{1+\frac{4 \xi_{i j 2}^{2}}{\nu}\right\}^{-\left(\frac{\nu+1}{2}\right)}\left[\frac{1}{4 \alpha^{2} \xi_{i j 1}^{2}}\right.\right.\right. \\
& \left.-w_{i j}\left(\xi_{i j 1}^{2}+\xi_{i j 2}^{2}\right)+\frac{w_{i j} 8 \xi_{i j 2}^{2} \xi_{i j 1}^{2}}{\left(\nu+4 \xi_{i j 2}^{2}\right)}\right]-\xi_{i j 1}\left(\frac{\nu+1}{2}\right)\left[1+\frac{4 \xi_{i j 2}^{2}}{\nu}\right]^{-\left(\frac{\nu+3}{2}\right)} \\
& {\left[-\frac{4 \xi_{i j 2} \xi_{i j 1}}{\nu}\right]\left[\frac{1}{4 \alpha^{2} \xi_{i j 1}^{2}}-w_{i j}\left(\xi_{i j 1}^{2}+\xi_{i j 2}^{2}\right)+\frac{w_{i j} 8 \xi_{i j 2}^{2} \xi_{i j 1}^{2}}{\left(\nu+4 \xi_{i j 2}^{2}\right)}\right] } \\
& +\xi_{i j 1}\left[1+\frac{4 \xi_{i j 2}^{2}}{\nu}\right]^{-\left(\frac{\nu+1}{2}\right)}\left[\frac{4 \alpha^{2} \xi_{i j 1} \xi_{i j 2}}{\left(4 \alpha^{2} \xi_{i j 1}^{2}\right)^{2}}-\frac{4 w \xi_{i j 2} \xi_{i j 1}}{\nu+4 \xi_{i j 2}^{2}}\left(\xi_{i j 1}^{2}+\xi_{i j 2}^{2}\right)\right. \\
& +2 w_{i j} \xi_{i j 1} \xi_{i j 2}+\frac{32 w_{i j} \xi_{i j 2}^{3} \xi_{i j 1}^{3}}{\left(\nu+4 \xi_{i j 2}^{2}\right)^{2}} \\
& \left.\left.\frac{-\left(\nu+4 \xi_{i j 2}^{2}\right) 8 w_{i j} \xi_{i j 2} \xi_{i j 1}\left(\xi_{i j 2}^{2}+\xi_{i j 1}^{2}\right)+4 \xi_{i j 2} \xi_{i j 1}\left(w_{i j} 8 \xi_{i j 2}^{2} \xi_{i j 1}^{2}\right)}{\left(\nu+4 \xi_{i j 2}^{2}\right)^{2}}\right]\right\} d y_{i j} \\
= & 0 .
\end{aligned}
$$

Portanto,

$$
\frac{\partial \mathbf{N}_{i}}{\partial \boldsymbol{\mu}_{i}}=0
$$




$$
\boldsymbol{\Omega}_{i}^{-1}=\operatorname{diag}\left\{\frac{1}{\mathrm{E}\left(v_{i j}^{2}\right)}, \ldots, \frac{1}{\mathrm{E}\left(v_{i s}^{2}\right)}\right\}=\operatorname{diag}\left\{\frac{\alpha_{i j}}{2}, \ldots, \frac{\alpha_{i s}}{2}\right\} \Rightarrow \frac{\partial \boldsymbol{\Omega}_{i}^{-1}}{\partial \boldsymbol{\alpha}}=\operatorname{diag}\left\{\frac{1}{2}, \ldots, \frac{1}{2}\right\}
$$

e

$$
\frac{\partial \mathbf{M}_{i}}{\partial \boldsymbol{\alpha}}=\operatorname{diag}\left(\frac{4}{\alpha_{i j}^{3}}, \ldots, \frac{4}{\alpha_{i s}^{3}}\right)
$$

Portanto,

$$
\mathrm{E}\left(\frac{\partial \boldsymbol{\zeta}_{i}(\boldsymbol{\theta})}{\partial \boldsymbol{\gamma}}\right)=\mathbf{Z}_{\mathbf{i}}^{\mathbf{T}} \mathbf{H}_{i} \mathbf{M}_{i} \boldsymbol{\Omega}_{i}^{-1} \mathbf{M}_{i} \mathbf{H}_{i} \mathbf{Z}_{i}
$$

A matriz de sensibilidade e a matriz de variabilidade para a equação de estimação generalizada (F.1) são, respectivamente, dadas por

$$
\begin{aligned}
\mathbf{S}_{\mathbf{i}}(\boldsymbol{\theta}) & =\mathrm{E}\left(\begin{array}{cc}
\frac{\partial}{\partial \boldsymbol{\beta}^{\top}} \mathbf{\Psi}_{i}(\boldsymbol{\beta}) & \frac{\partial}{\partial \boldsymbol{\gamma}^{\top}} \mathbf{\Psi}_{i}(\boldsymbol{\beta}) \\
\frac{\partial}{\partial \boldsymbol{\beta}^{\top}} \boldsymbol{\zeta}_{i}(\boldsymbol{\gamma}) & \frac{\partial}{\partial \boldsymbol{\gamma}^{\top}} \boldsymbol{\zeta}_{i}(\boldsymbol{\gamma})
\end{array}\right) \\
& =\left(\begin{array}{cc}
\mathbf{X}_{\mathbf{i}}^{\top} \mathbf{N}_{i} \boldsymbol{\Sigma}_{i}^{-1 / 2} \mathbf{R}(\rho)^{-1} \boldsymbol{\Sigma}_{i}^{-1 / 2} \mathbf{N}_{i} \mathbf{X}_{i} . & \mathbf{0} \\
\mathbf{0} & \mathbf{Z}_{\mathbf{i}}^{\mathbf{T}} \mathbf{H}_{i} \mathbf{M}_{i} \boldsymbol{\Omega}_{i}^{-1} \mathbf{M}_{i} \mathbf{H}_{i} \mathbf{Z}_{i} .
\end{array}\right) \\
& =\mathbf{Q}_{\mathbf{i}}^{\top} \boldsymbol{\Lambda}_{i} \boldsymbol{\Upsilon}_{i}^{-1} \boldsymbol{\Lambda}_{i} \mathbf{Q}_{\mathbf{i}},
\end{aligned}
$$

$\log 0$

$$
\sum_{i=1}^{n} \mathbf{S}_{\mathbf{i}}(\boldsymbol{\theta})=\sum_{i=1}^{n} \mathrm{E}\left\{\mathbf{Q}_{\mathbf{i}}^{\top} \boldsymbol{\Lambda}_{i} \mathbf{\Upsilon}_{i}^{-1} \mathbf{d}_{i} \mathbf{d}_{i}^{\top}\right\}=\sum_{i=1}^{n} \mathbf{Q}_{\mathbf{i}}^{\top} \boldsymbol{\Lambda}_{i} \mathbf{\Upsilon}_{i}^{-1} \mathrm{E}\left\{\mathbf{d}_{i} \mathbf{d}_{i}^{\top}\right\} \boldsymbol{\Upsilon}_{i}^{-T} \boldsymbol{\Lambda}_{i}^{\top} \mathbf{Q}_{\mathbf{i}}
$$

\section{F.2.2 Influência conformal sob heterogeneidade do parâmetro de forma}

i) Perturbação da variável resposta

Considerando a perturbação da variável resposta

$$
y_{\omega i j}=y_{i j}+\omega_{i j} s_{y_{i j}}
$$

temos que

$$
\boldsymbol{\Gamma}(\boldsymbol{\theta} \mid \boldsymbol{\omega})=\mathbf{Q}^{\top} \mathbf{W} \boldsymbol{\Lambda}^{-1} \mathbf{d}_{\omega}
$$

em que $\mathbf{d}_{\omega}=\left(\mathbf{d}_{\omega 1}^{\top}, \ldots, \mathbf{d}_{\omega n}^{\top}\right)^{\top}, \operatorname{com} \mathbf{d}_{\omega i}=\left(d_{\omega i 1}^{\top}, \ldots, d_{\omega i s}^{\top}\right)^{\top}$

$$
\boldsymbol{\Delta}=\frac{\partial \boldsymbol{\Gamma}(\boldsymbol{\theta} \mid \boldsymbol{\omega})}{\partial \boldsymbol{\omega}^{\top}}=\mathbf{Q}^{\top} \mathbf{W} \boldsymbol{\Lambda}^{-1} \frac{\partial \mathbf{d}_{\omega}}{\partial \boldsymbol{\omega}^{\top}}
$$

avaliado em $\hat{\boldsymbol{\theta}}$ e em $\boldsymbol{\omega}_{0}$. O vetor perturbado na variável resposta para $i$-ésima unidade experimental é $\mathbf{d}_{\omega i}=\left(\mathbf{u}_{\omega i}^{\top}, \mathbf{v}_{\omega_{i}}^{\top}\right)^{\top}$, em que $\mathbf{u}_{\omega i}=\left(u_{\omega i 1}, \ldots, u_{\omega i s}\right)^{\top}$ e $\mathbf{v}_{\omega i}=\left(v_{\omega i 1}, \ldots, v_{\omega i s}\right)^{\top}$, com $u_{\omega i j}$ definido em 
(3.8) com derivada em relação a $\omega_{i j}$ dada em (3.9).

E temos que,

$$
v_{\omega i j}=\frac{1}{\alpha_{i j}}\left[4 w_{\omega i j} \xi_{\omega i j 2}^{2}-1\right]
$$

em que $w_{\omega i j}=\frac{\nu+1}{\nu+4 \xi_{\omega i j 2}^{2}}$ e $\xi_{\omega i j 2}=\frac{1}{\alpha} \operatorname{senh}\left(\frac{y_{\omega i j}-\mu_{i j}}{2}\right)$ e cujas as derivadas em relação a $\omega_{i j}$ é dada por,

$$
\begin{gathered}
\frac{\partial \xi_{\omega i j 2}}{\partial \omega_{i j}}=\frac{1}{\alpha} \cosh \left(\frac{y_{\omega i j}-\mu_{i j}}{2}\right) \frac{1}{2}=\frac{\xi_{\omega i j 1}}{2} \\
\frac{\partial w_{\omega i j}}{\partial \omega_{i j}}=-s_{y_{i j}} \frac{(\nu+1)\left(4 \xi_{\omega i j 2} \xi_{\omega i j 1}\right)}{\left(\nu+4 \xi_{\omega i j 2}^{2}\right)^{2}}=-w_{\omega i j} \frac{\left(4 \xi_{\omega i j 2} \xi_{\omega i j 1}\right)}{\nu+4 \xi_{\omega i j 2}^{2}} s_{y_{i j}}
\end{gathered}
$$

em que $\xi_{\omega i j 1}=\frac{1}{\alpha} \cosh \left(\frac{y_{\omega i j}-\mu_{i j}}{2}\right)$. Portanto,

$$
\begin{aligned}
\frac{\partial v_{\omega_{i j}}}{\partial \omega_{i j}} & =\frac{4}{\alpha} \frac{\partial w_{\omega i j}}{\partial \omega_{i j}} \xi_{i j 1}^{2}+s_{y_{i j}} \frac{4}{\alpha} w_{\omega i j} \xi_{\omega i j 2} \xi_{\omega i j 1} \\
& =s_{y_{i j}} \frac{4}{\alpha} w_{\omega i j} \xi_{\omega i j 2} \xi_{\omega i j 1}\left[1-\frac{\xi_{\omega i j 2}^{2}}{\nu+4 \xi_{\omega i j 2}^{2}}\right]
\end{aligned}
$$

De forma que,

$$
\frac{\partial \mathbf{d}}{\partial \boldsymbol{\omega}^{\top}}=\left(\begin{array}{c}
\dot{\mathcal{U}} \\
\dot{\mathcal{V}}
\end{array}\right),
$$

$\operatorname{com} \dot{\mathcal{U}}=\operatorname{diag}\left(\dot{\mathcal{U}}_{1}, \ldots, \dot{\mathcal{U}}_{n}\right)$ e $\dot{\mathcal{V}}=\operatorname{diag}\left(\dot{\mathcal{V}}_{1}, \ldots, \dot{\mathcal{V}}_{n}\right)$ em que $\dot{\mathcal{U}}_{i}=\operatorname{diag}\left(\frac{\partial u_{\omega i 1}}{\partial \omega_{i 1}}, \ldots, \frac{\partial u_{\omega i s}}{\partial \omega_{i s}}\right)$ e $\dot{\mathcal{V}}_{i}=$ $\operatorname{diag}\left(\frac{\partial v_{\omega i 1}}{\partial \omega_{i 1}}, \ldots, \frac{\partial v_{\omega i s}}{\partial \omega_{i s}}\right)$. 
Apêndice G

Tabelas referentes às simulações do

Capítulo 4 


\begin{tabular}{|c|c|c|c|c|}
\hline & 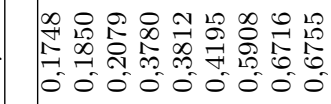 & 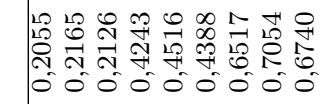 & 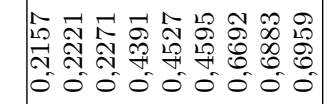 & 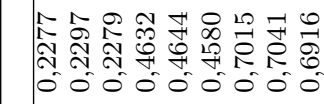 \\
\hline & 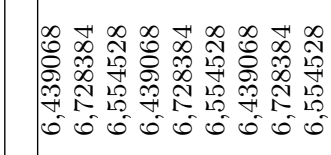 & 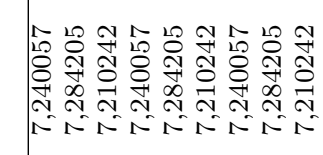 & 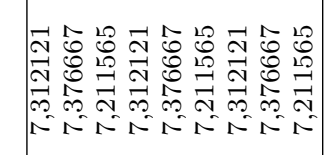 & 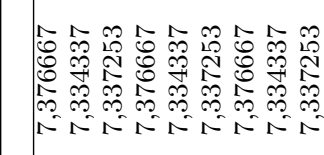 \\
\hline & 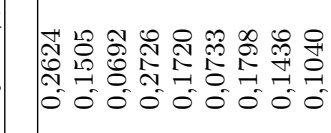 & 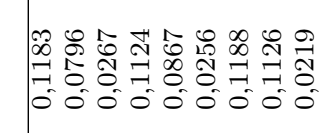 & 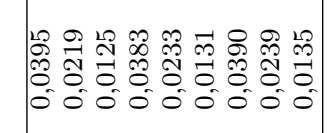 & 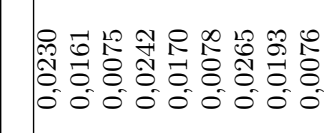 \\
\hline & 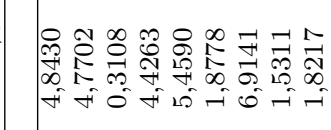 & 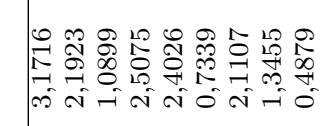 & 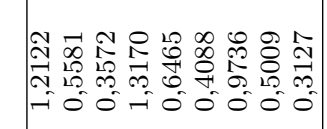 & 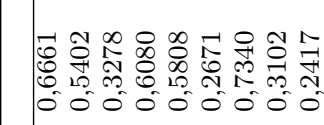 \\
\hline & 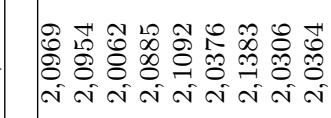 & 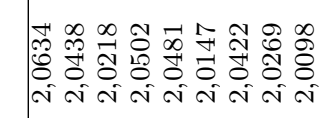 & 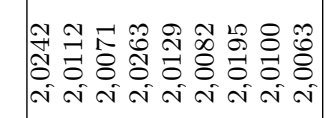 & 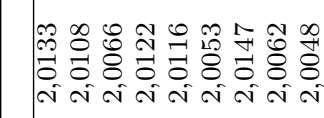 \\
\hline & 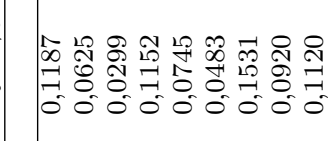 & 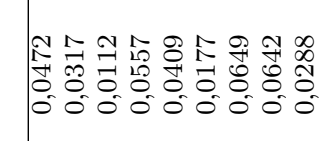 & 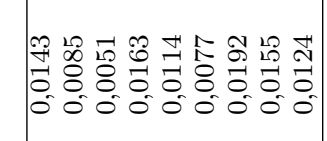 & 年 \\
\hline & 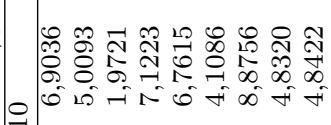 & 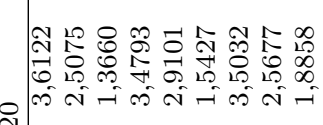 & 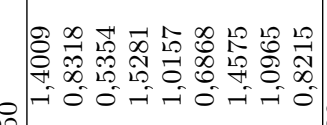 & $\mid \begin{array}{ll}1 \\
0 \\
0 \\
0 \\
0 \\
0\end{array}$ \\
\hline & 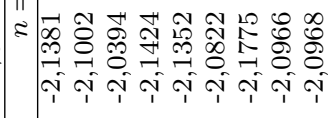 & 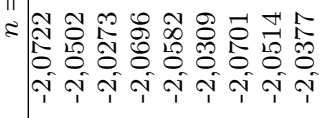 & $=\left[\begin{array}{ll}0 \\
0 \\
0 \\
0\end{array}\right.$ & $=8$ \\
\hline & 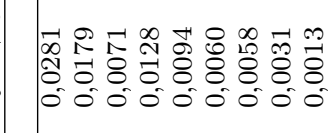 & 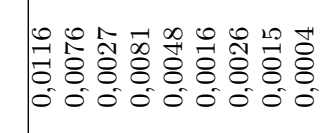 & 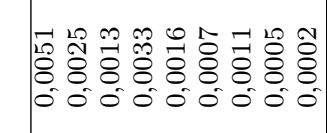 & 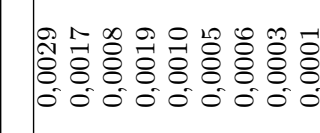 \\
\hline & 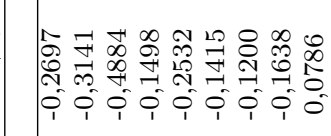 & 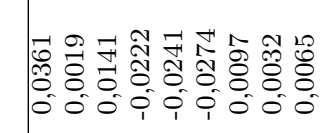 & 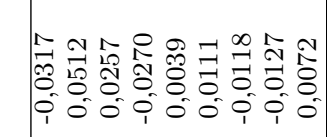 & 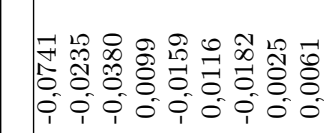 \\
\hline$\vec{b}$ & 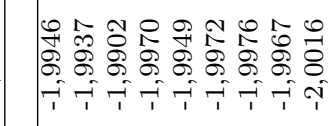 & 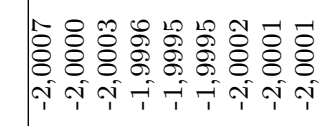 & 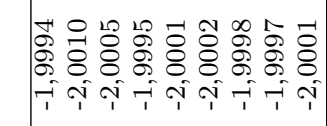 & \\
\hline & 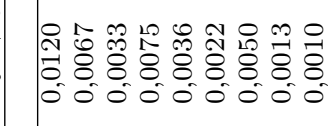 & 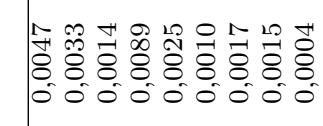 & 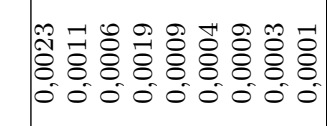 & $\begin{array}{l}7 \\
\overline{8} \\
0 \\
0\end{array}$ \\
\hline 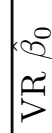 & 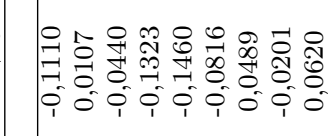 & 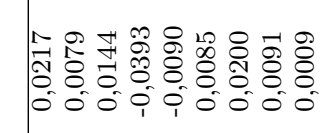 & 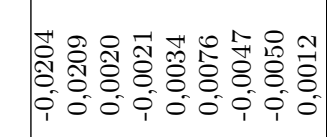 & \\
\hline & 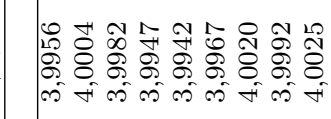 & 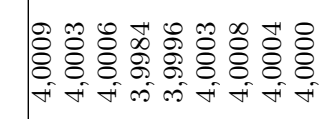 & 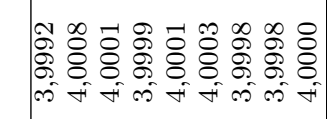 & \\
\hline is & 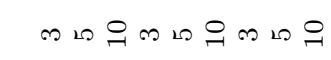 & 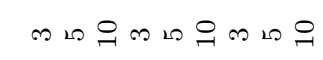 & 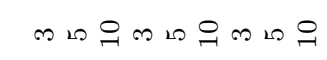 & $m \infty 9 \infty n$ \\
\hline & : & 0 & $\because$ & 0 \\
\hline
\end{tabular}


|

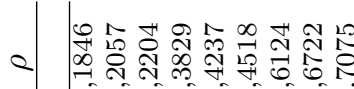
ond 0000000

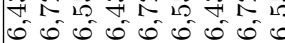

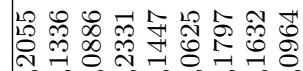

$\begin{array}{llll} & 0 \\ 0 & 0 & 0 & 0\end{array}$

¿

$>$ भy

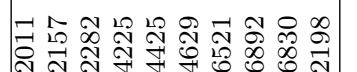

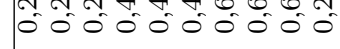

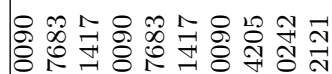

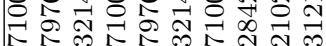

o on 0

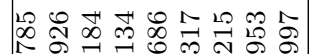

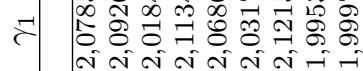

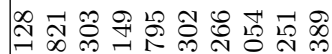

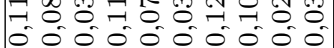

ปี่

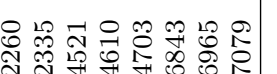

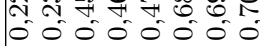

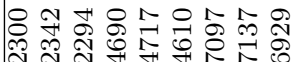
人织

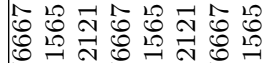

贾

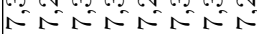

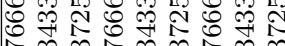

票前

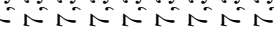

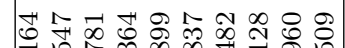

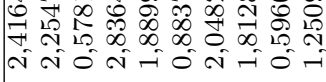

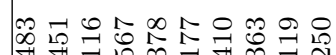

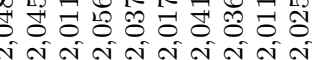

象

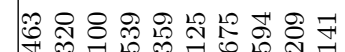
00000000

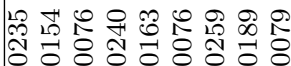

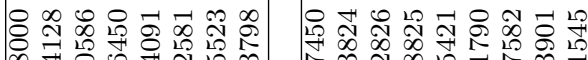
$\begin{array}{lllll}0 & 0 \\ 0 & 0 & 0 & 0 & 0\end{array}$ O 000000000 0000000000

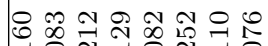

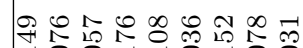

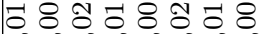

$0880-108080$

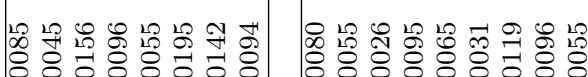

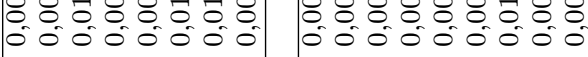

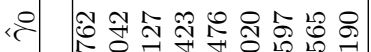

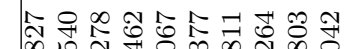

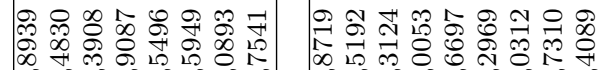

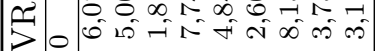

$m_{0}$

e

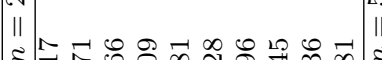
000 is

$\sum^{2}$

तิ

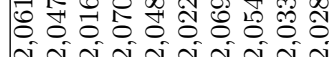

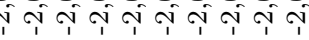

2

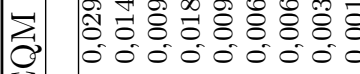

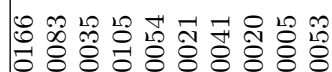

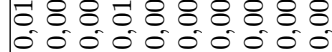

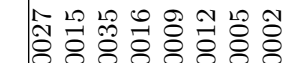
$\infty$

=

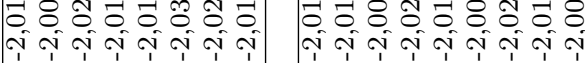

赵

$>$ 年

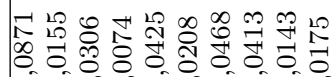

în

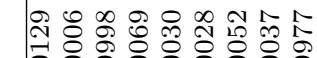

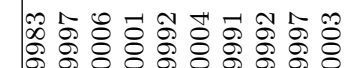

ल

वरन

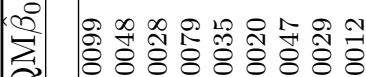

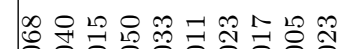
$0,0000^{\circ} 0^{\circ} 0^{\circ}$

융

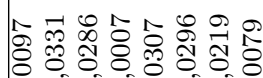
-

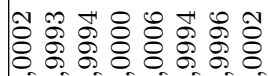

भit 00000000

o 000000000

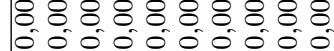

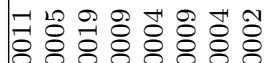

00000000

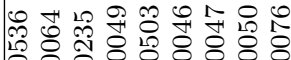
- o i i i i i i

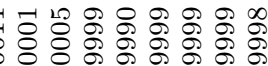

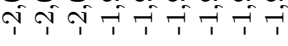

œ

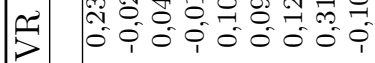

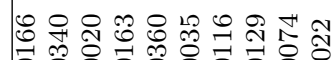

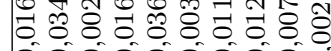

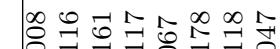

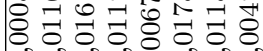

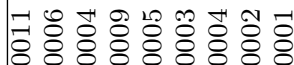
$\begin{array}{lllll}0 & 0 & 0 & 0 & 0\end{array}$

일 1 1 1 1 1 iq ílói

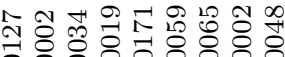

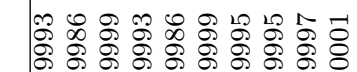

응용횽용ㅇㅇㅇ용용용 $\begin{array}{lllll}0 & 0 & 0 & 0 & 0 \\ 0 & 0 & 0 & 0 \\ 0 & 0 & 0 & 0 \\ 0\end{array}$

त㧼

$\ddot{\sim} \stackrel{8}{0}$

ปร

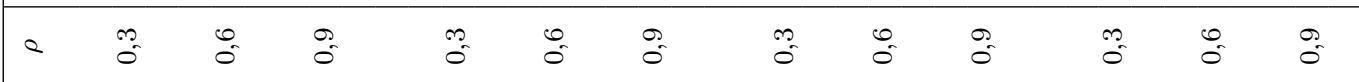




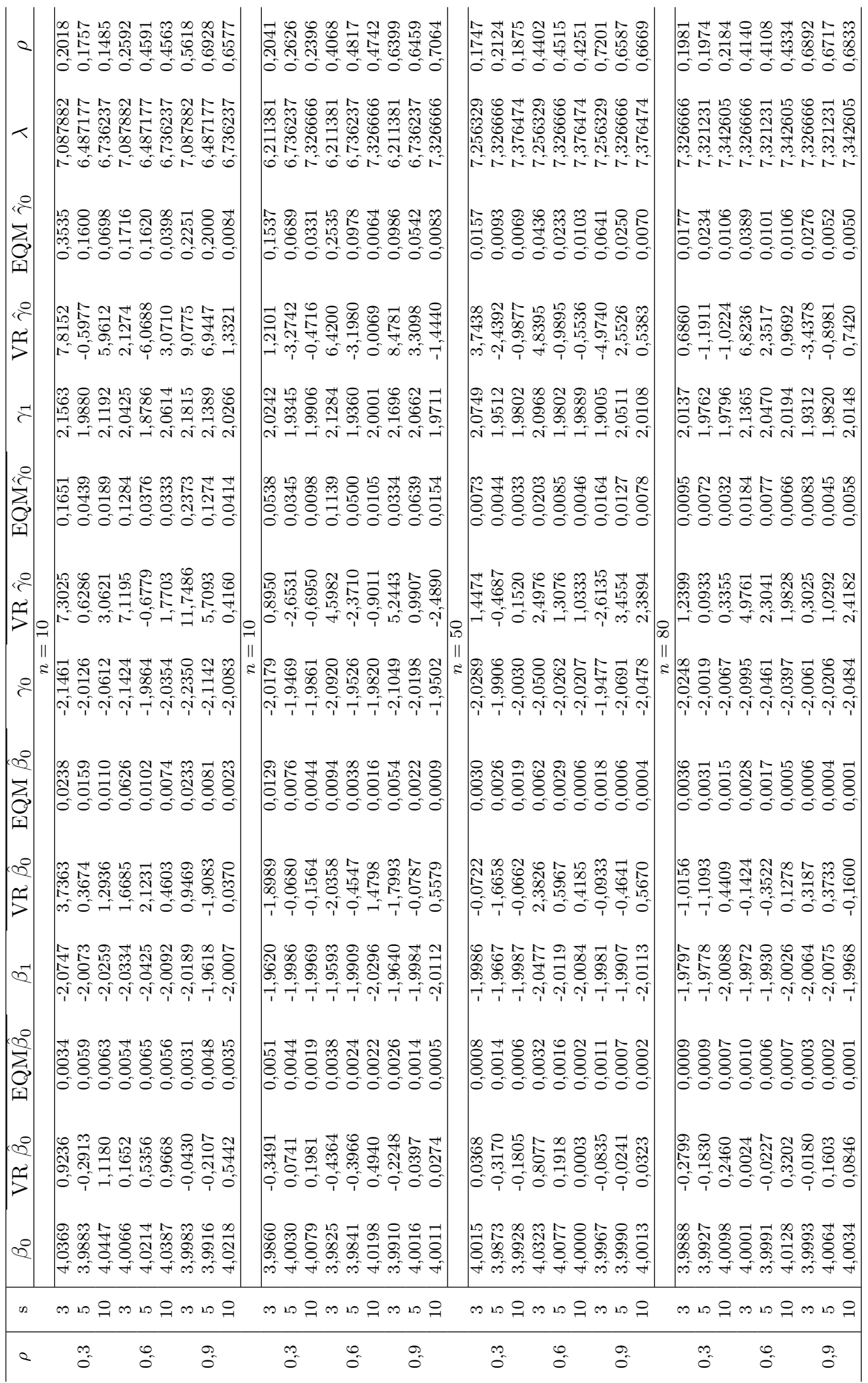




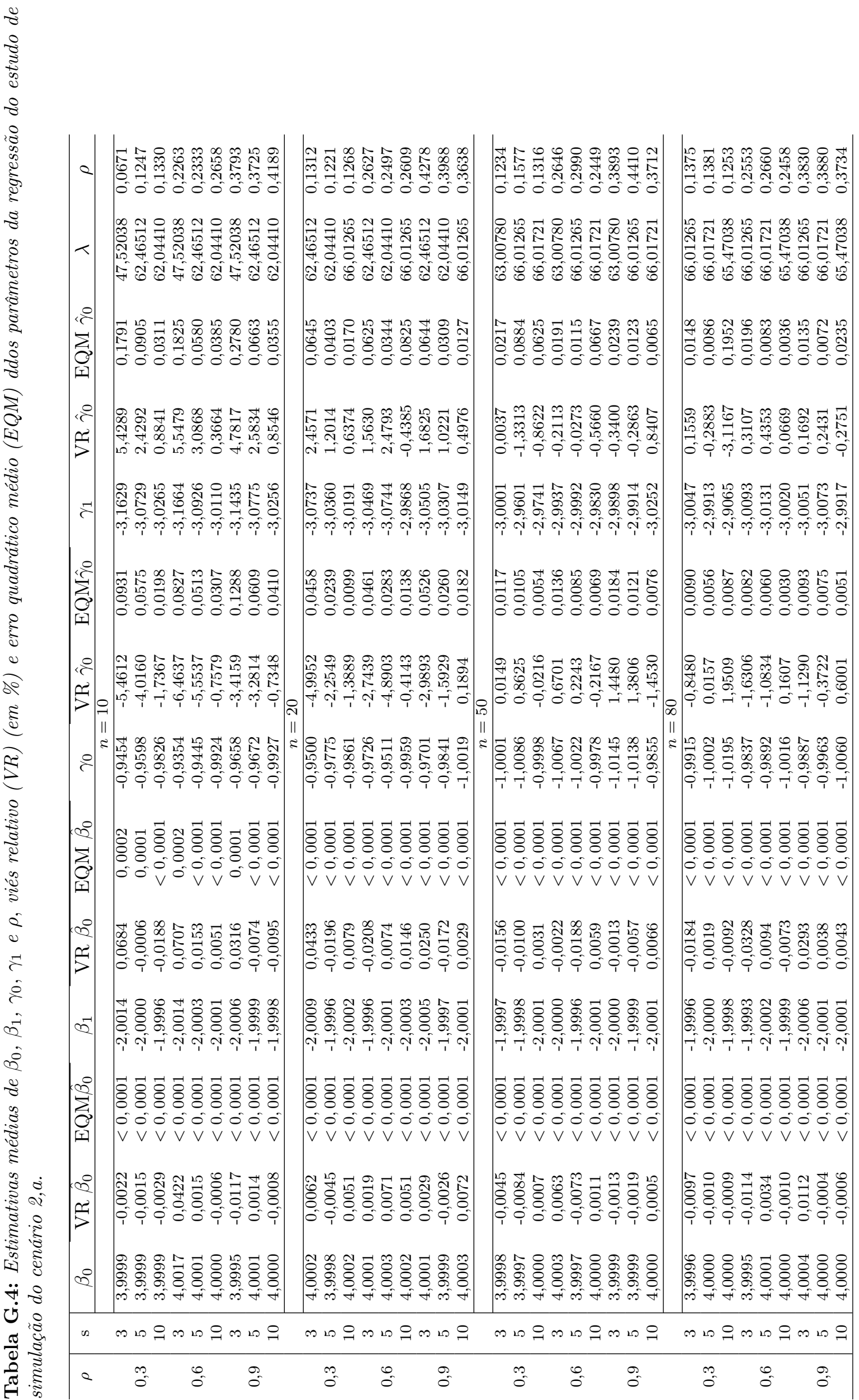




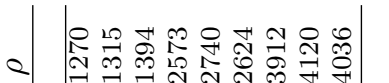

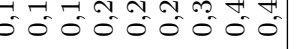

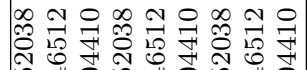

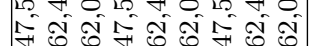

点 สิ

가이

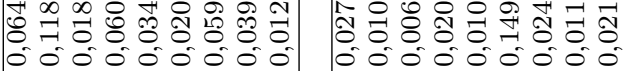

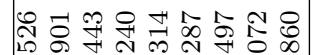

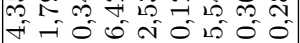

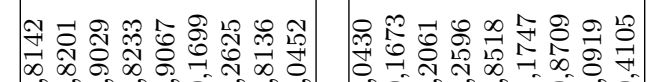

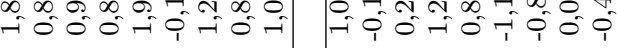

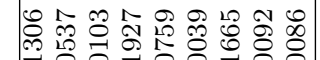

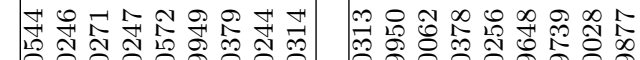

की

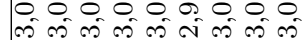

i

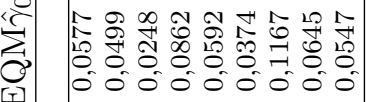

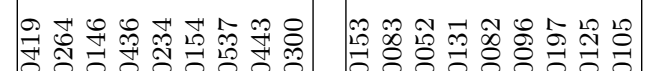

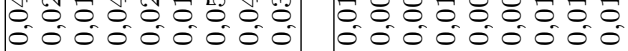

田

<

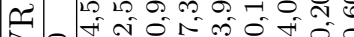

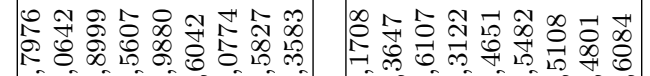

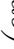

s

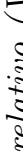
2

일

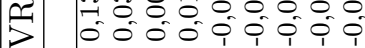

次 ำ $\vee \vee \vee V \vee V \vee V \vee$

눙

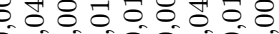

正 \&

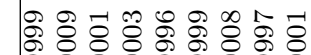

N-1 พิ

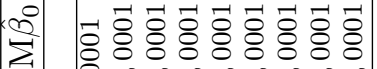

O

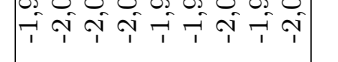

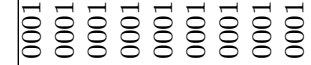

0000 00000

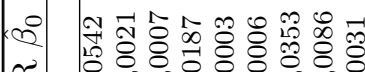

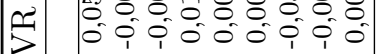

$V V V V V V V V$

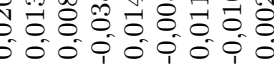

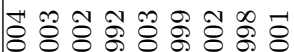

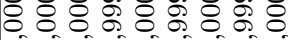
भิ

害产 0 0000000

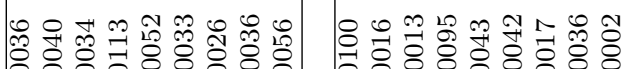

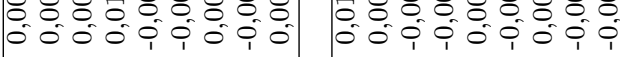

ค

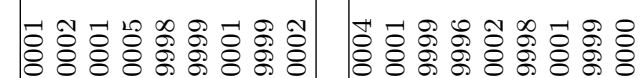

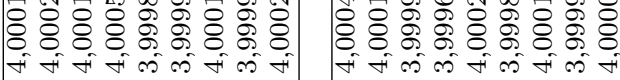

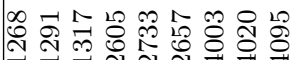

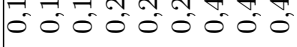

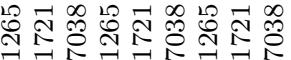

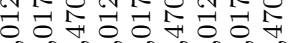
$8 \%$ \& 80808080

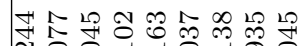

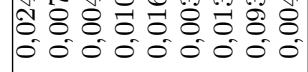

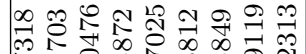

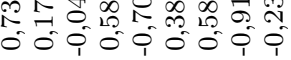

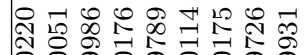

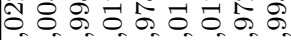

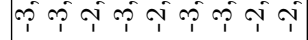

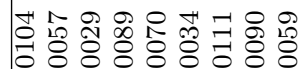
$\begin{array}{ccccc}0 & 0 & 0 & 0 & 0 \\ 0 & 0 & 0 & 0 & 0 \\ 0 & 0 & 0\end{array}$

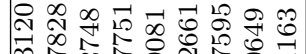
inc II

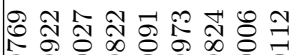

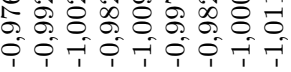

家 $\begin{array}{llllllllll}0 & 0 & 0 & 0 & 0 & 0 & 0 & 0 & 0 & 0 \\ 0\end{array}$ $\vee \vee \vee \vee \vee \vee \vee \vee V ~$

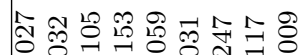

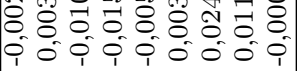

\& 굥 \& in a th Th 害宫宫

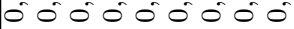
$\vee \vee \vee \vee \vee \vee \vee \vee V$

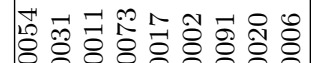

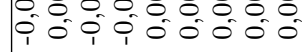

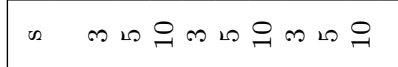

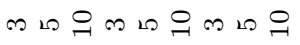

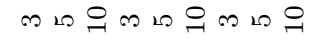

\&

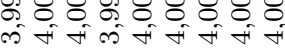

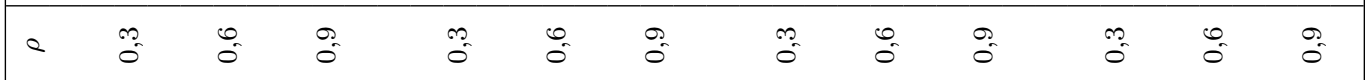




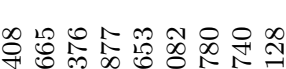

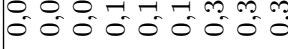

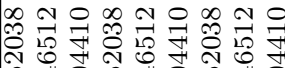

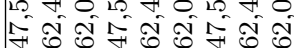

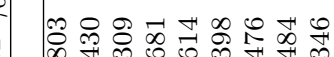

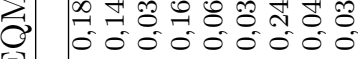

ำ

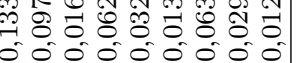

돗 -

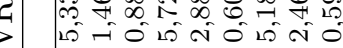

$8 \infty \wedge ⿻$ 하

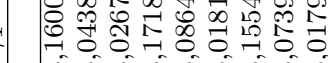
$p_{i} p_{i} p_{i} p_{i}^{p} p_{i}^{p} p_{i}^{p} p_{i}^{p}$

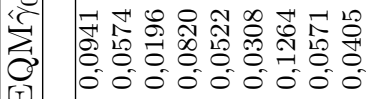

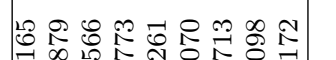

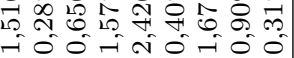

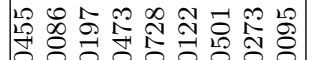
Pि

ஜि พิ

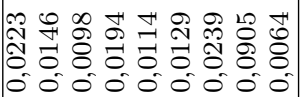

12 $\infty$

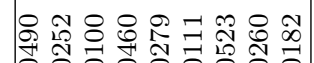

구용

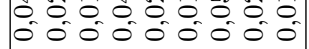

$\begin{array}{llllll} & 0 & 0 & 0 & 0 & 0\end{array}$

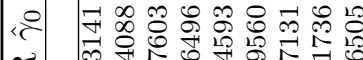

$>0$ la

है

$\stackrel{2}{5}$

.

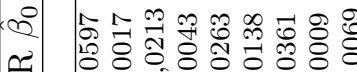

$>00.000000$

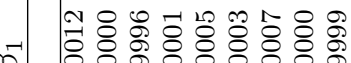

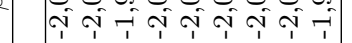

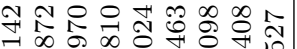

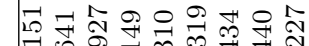

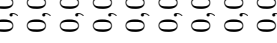

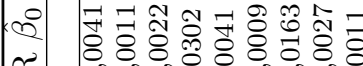

$\$$ i. i०

है.

雨

$\ddot{\theta}$ 요

ن்

䨔

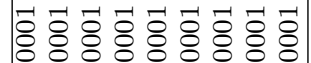

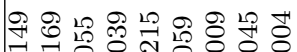

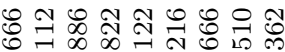

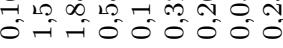

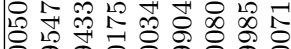

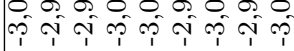

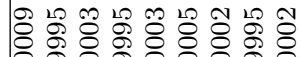

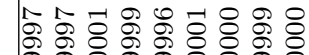

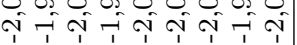

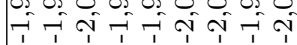

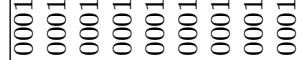

\section{0}

हुํㅡ응

o 000000000

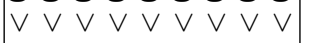
$\checkmark \vee \vee \vee \vee \vee \vee \vee V$

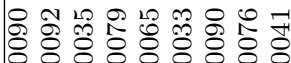

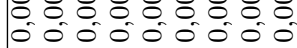

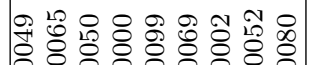

qgen

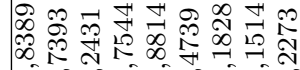




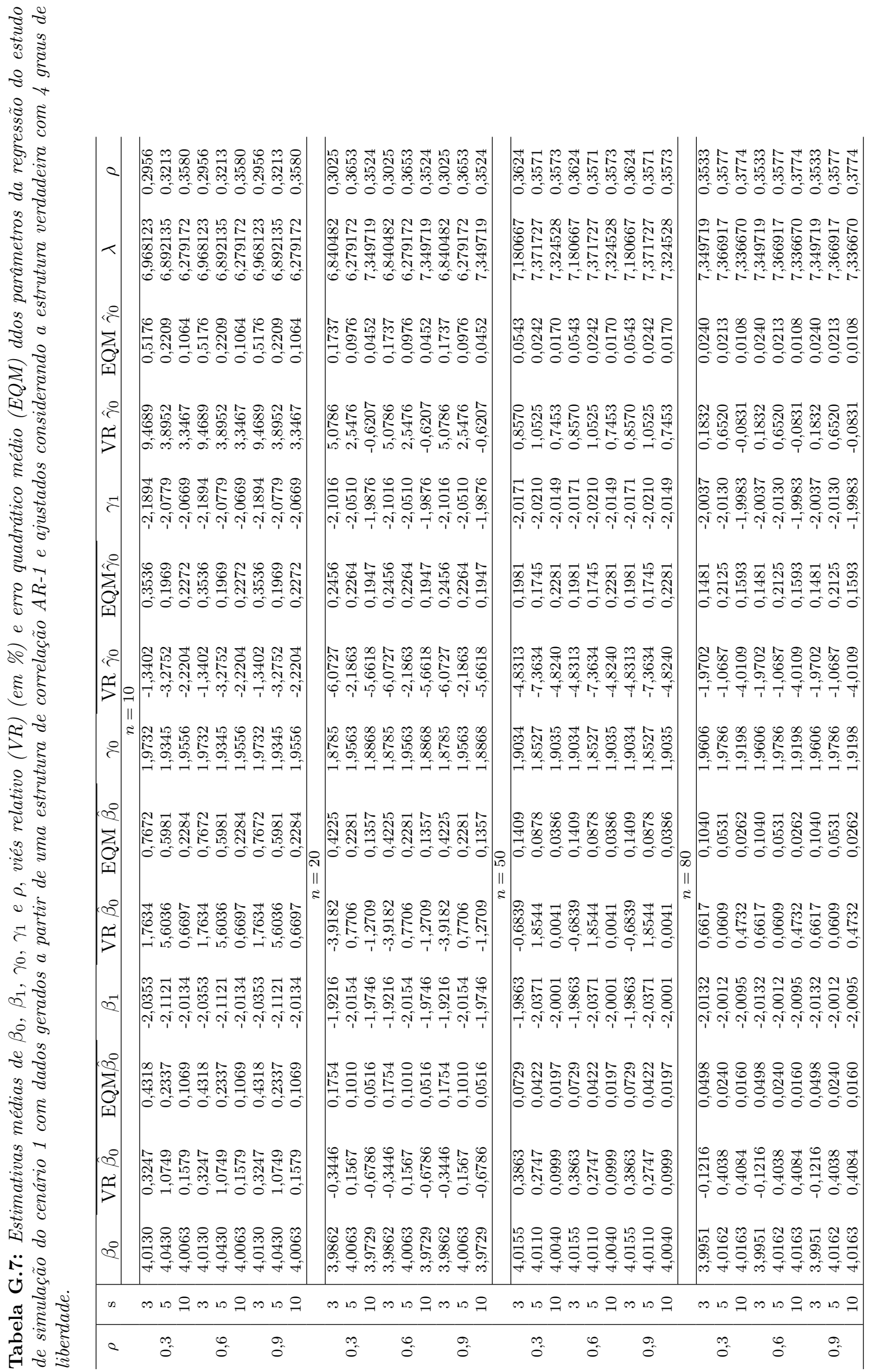




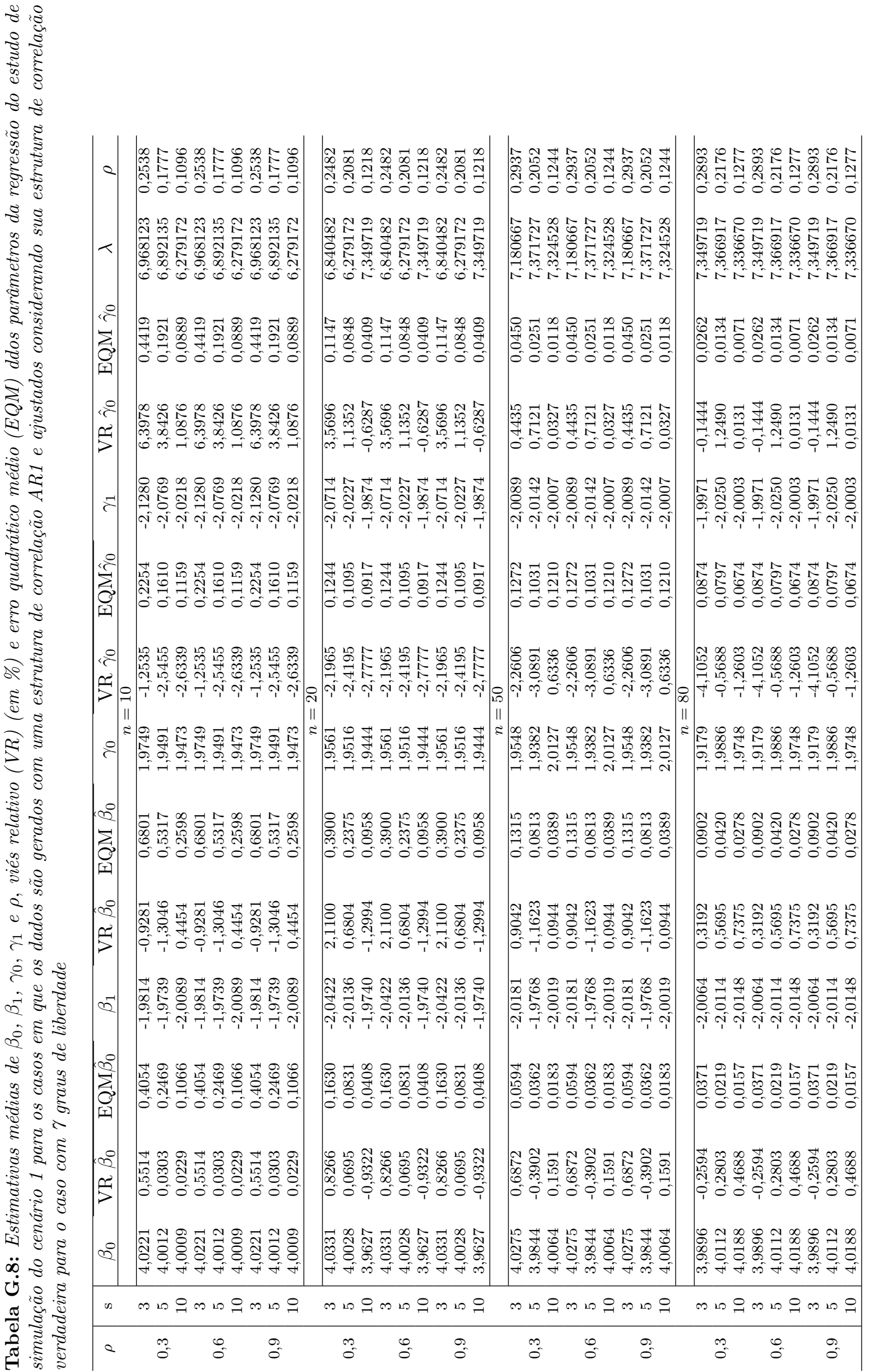




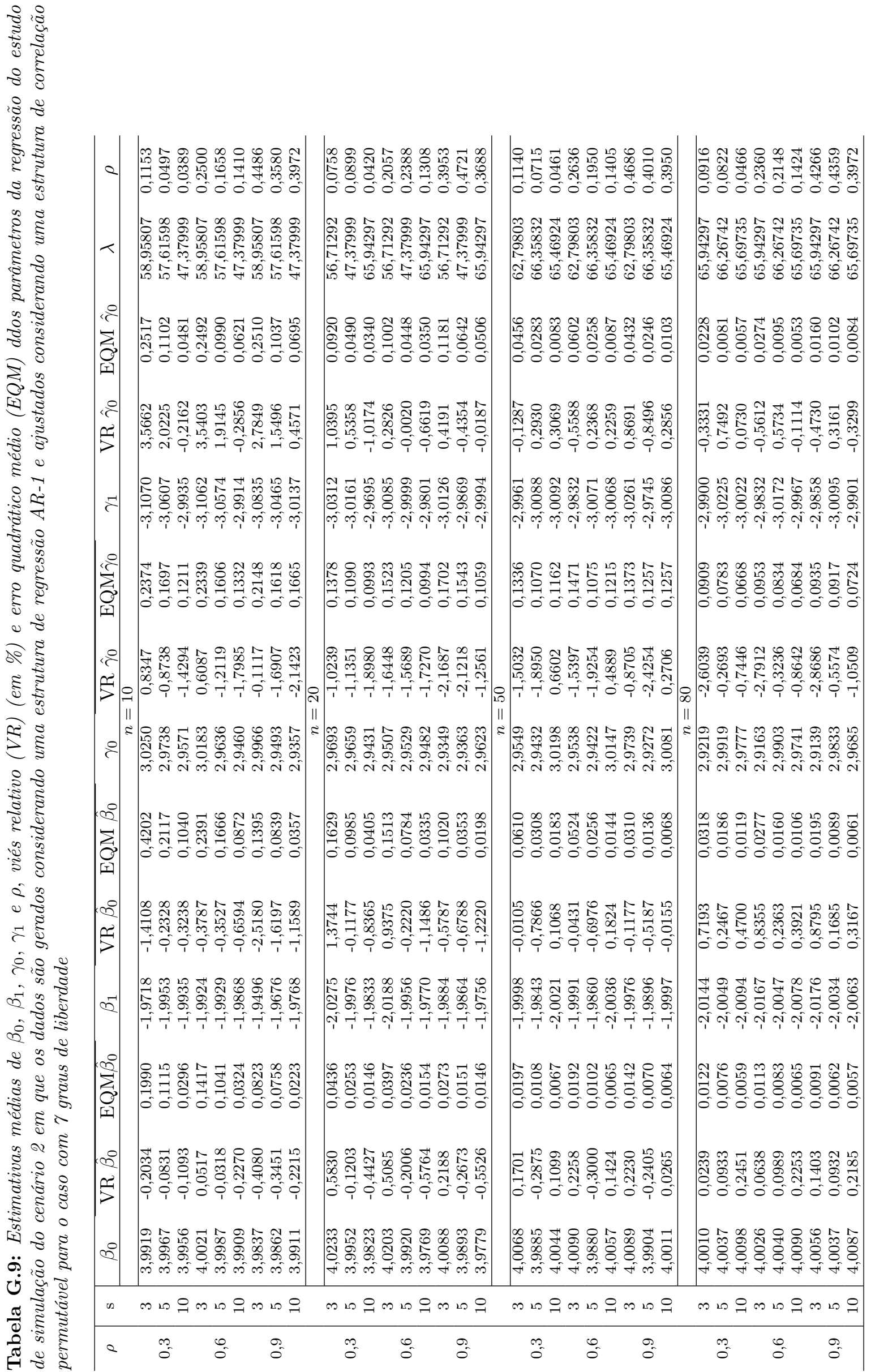




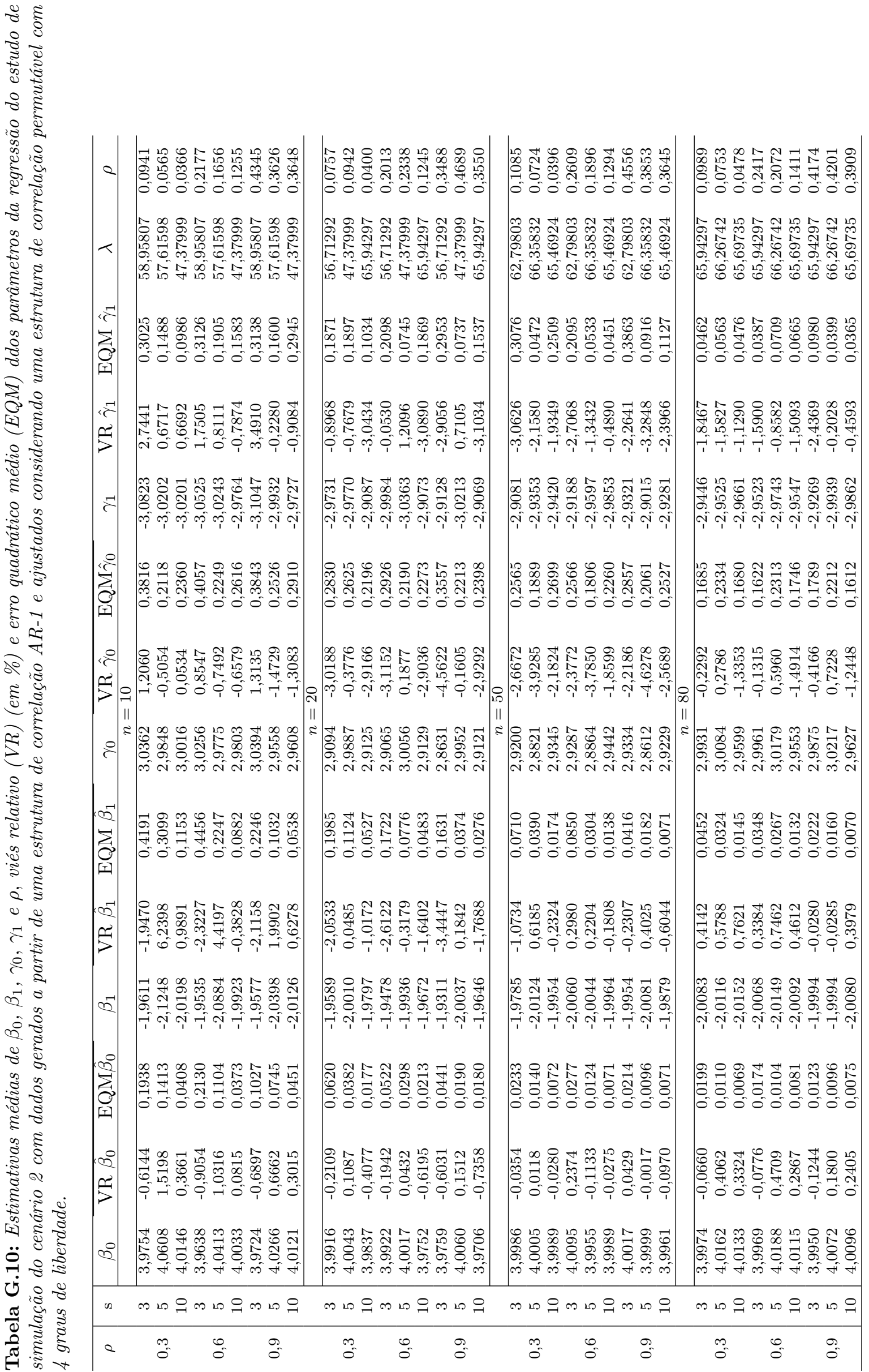




\section{Referências Bibliográficas}

Alencar et al.(2012) A.P. Alencar, J.M. Singer e F.M.M. Rocha. Competing regression models for longitudinal data. Biometrical Journal, 54:214-229. Citado na pág. 2, 14

Artes(1997) R. Artes. Extensões da Teoria das Equações de Estimação Generalizadas a Dados Circulares e Modelos de Dispersão. Tese de Doutorado, Instituto de Matemática e Estatística, Universidade de São Paulo, Brasil. Citado na pág. 2, 11, 12, 17, 21, 22

Artes et al.(2000) R. Artes, G.A. Paula e R. Ranvaud. Analysis of circular longitudinal data based on generalized estimating equations. Australian and New Zealand Journal of Statistics, 42: 347-358. Citado na pág. 11, 13

Artes e Botter(2005) Rinaldo Artes e Denise Aparecida Botter. Funções de estimação em modelos de regressão. Em $9^{a}$ Escola de Modelos de Regressão -ABE, São Pedro, SP. Citado na pág. 3

Balakrishnan et al.(2007) N. Balakrishnan, V. Leiva e Jorge Lopez. Acceptance sampling plans from truncated life tests based on the generalized birnbaum-saunders distribution. Communications in Statistics - Simulation and Computation, 36(3):643-656. Citado na pág. 2

Barnhart e Williamson(1998) H. X. Barnhart e J. M. Williamson. Goodness-of-fit tests for gee modeling with binary responses. Biometrics, 54(2):720-729. Citado na pág. 24

Barros et al.(2008) M. Barros, G.A. Paula e V. Leiva. A new class of survival regression models with heavy-tailed errors: robustness and diagnostics. Lifetime Data Analysis, 14:316-32. Citado na pág. $1,8,39$

Bhatti(2010) Chad R. Bhatti. The birnbaum-saunders autoregressive conditional duration model. Mathematics and Computers in Simulation, 80(10):2062 - 2078. Citado na pág. 2

Birnbaum e Saunders(1969) Z. W. Birnbaum e S. C. Saunders. A new family of life distributions. Journal of Applied Probability, 6:319-327. Citado na pág. 1, 3, 4

Cadigan e Farrell(2002) N.G. Cadigan e P.J. Farrell. Generalized local influence with applications to fish stock cohort analysis. J. Appl. Stat., 51:469-483. Citado na pág. 29

Cook(1986) R.D. Cook. Assessment of local influence. J. R. Stat. Soc., 5:133-169. Citado na pág. 29

Cook(1977) R.D. Cook. Detection of influential observations in linear regressions. Technometrics, 19:15-18. Citado na pág. 28

Crowder(1987) M. Crowder. On linear and quadratic estimating functions. Biometrika, 74: 591-597. Citado na pág. 13

Desmond(1985) A. Desmond. Stochastic models of failure in random environments. Canad. J. Statist., 13:171-183. Citado na pág. 1

Desmond(1986) A. Desmond. On the relationship between two fatigue-life models. IEEE Transactions on Reliability, 35:167-169. Citado na pág. 1 
Desmond et al.(2012) A. F. Desmond, C. L. Cíntora-González, R. S. Singh e X. Lu. A mixed effects log-linear model based on the birnbaum-saunders distribution. Computational Statistics and Data Analysis, 56:399-407. Citado na pág. 17

Díaz-García e Domínguez-Molina(2006) J. A. Díaz-García e J.R. Domínguez-Molina. Some generalisations of birnbaum-saunders and sinh-normal distributions. Intern. Math. Forum, 1: 1709-1727. Citado na pág. 7

Díaz-García e Leiva(2005) J. A. Díaz-García e V. Leiva. A new family of life distributions based on the contoured elliptically distributions. Journal of Statistical Planning and Inference, 128:88-95. Citado na pág. 1, 5, 6, 7

Firth(1992) D. Firth. Discussion of "multivariate regression analysis for categorical data"by liang, zeger and qaqish. Journal of the Royal Statistical Society B, 54:24-26. Citado na pág. 22

Galea et al.(2004) M. Galea, V. Leiva e G. A. Paula. Influence diagnostics in log-birnbaumsaunders regression models. Journal of Applied Statistics, 31:1049-1064. Citado na pág. 1, 8, 9, 53

Godambe(1997) V. P. Godambe. Estimating Functions: A Synthesis of Least Squares and Maximum Likelihood Methods. Selected Proceedings of the Symposium on Estimating Functions, 5-16, Institute of Mathematical Statistics, Hayward, CA. Citado na pág. 3, 11, 13

Greene(2012) W.H. Greene. Econometric Analysis. Prentice Hall, New York. Citado na pág. 32

Horton et al.(1999) Nicholas J. Horton, Judith D. Bebchuk, Cheryl L. Jones, Stuart R. Lipsitz, Paul J. Catalano, Gwendolyn E. P. Zahner e Garrett M. Fitzmaurice. Goodness-of-fit for gee: an example with mental health service utilization. Statistics in Medicine, 18(2):213-222. Citado na pág. 2,24

Johnson(1949) N. L. Johnson. Systems of frequency curves generated by methods of translation. Biometrika, 36:149-176. Citado na pág. 5

Johnston(1996) G. Johnston. Repeated measures analysis with discrete data using the sas system, sas institute inc. http://academic.son.wisc.edu/rdsu/pdf/gee.pdf, 1996. Citado na pág. 22

Jørgensen e Labouriau(1994) B. Jørgensen e R. S. Labouriau. Exponential Families and Theoretical Inference. Lectures Notes, Department of Statistics, University of British Columbia. Citado na pág. 12

Jørgensen et al.(1996) B. Jørgensen, S. Lundbye-Christensen, P. X-K. Song e L. Sun. State-space models for multivariate longitudinal data of mixed types. The Canadian Journal of Statistics / La Revue Canadienne de Statistique, 24(3):385-402. Citado na pág. 20

Kundu et al.(2010) D. Kundu, N. Balakrishnan e A. Jamalizadeh. Bivariate birnbaum-saunders distribution and its associated inference. Journal of Multivariate Analysis, 101:113-125. Citado na pág. 10

Kundu et al.(2013) D. Kundu, N. Balakrishnan e A. Jamalizadeh. Multivariate birnbaum-saunders distribution; properties, inference and a generalization. Journal of Multivariate Analysis, 101: 113-125. Citado na pág. 2, 10, 14, 23, 43

Lee e Qaqish(2004) J. H. Lee e B. F. Qaqish. Modified gee and goodness of the marginal fit (gomf) test with correlated binary responses for contingency tables. Biometrical Journal, 46: 675-686. Citado na pág. 24

Leiva(2016) V. Leiva. The Birnbaum-Saunders distribution. Elsevier. Citado na pág. 1, 3 
Leiva et al.(2007) V. Leiva, M. K. Barros, G. A. Paula e M. Galea. Influence diagnostics in log-birnbaum-saunders regression models with censored data. Computational Statistics and Data Analysis, 51:5694-5707. Citado na pág. 1, 8, 17

Leiva et al.(2008) V. Leiva, M. Barros, G. A. Paula e A. Sanhueza. Generalized birnbaum-saunders distributions applied to air pollutant concentration. Environmetrics, 19(3):235-249. Citado na pág. 2

Leiva et al.(2014) V. Leiva, F. M. Santos-Neto, F.J.A. Cysneiros e M. Barros. Birnbaum-saunders statistical modelling: a new approach. Revstat Statistical Journal, 14:21-48. Citado na pág. 1

Lemonte e Cordeiro(2009) A. J. Lemonte e G. M. Cordeiro. Birnbaum-saunders nonlinear regression models. Comput. Stat. Data Anal, 53:4441-4452. Citado na pág. 8, 9

Lemonte e Patriota(2011) A. J. Lemonte e A. G. Patriota. Influence diagnostics in birnbaumsaunders nonlinear regression models. Journal of Applied Statistics, 38(5):871-884. Citado na pág. 9

Li et al.(2012) A.P. Li, Z.X. Chen e F.C. Xie. Diagnostic analysis for heterogeneous log-birnbaumsaunders regression models. Statistics \& Probability Letters, 82:1690-1698. Citado na pág. 53, 54

Liang e Zeger(1986) K-Y Liang e S.L. Zeger. Longitudinal analysis using generalized linear models. Biometrika, 73:13-22. Citado na pág. 2, 3, 11, 19, 21, 42

Manghi et al.(2016) R.F. Manghi, G.A. Paula e J.F.A. Cysneiros. On elliptical multilevel models. Journal of Applied Statistics, 43:2150-2171. Citado na pág. 32

Marchant et al.(2013) C. Marchant, K. Bertin, V. Leiva e H. Saulo. Generalized birnbaumsaunders kernel density estimators and an analysis of financial data. Computational Statistics $\mathbb{E}$ Data Analysis, 63:1-15. Citado na pág. 2

Marchant et al.(2016a) C. Marchant, V. Leiva e F.J.A. Cysneiros. A multivariate log-linear model for birnbaum-saunders distributions. IEEE Transactions on Reliability, 65:816-827. Citado na pág. $2,10,14$

Marchant et al.(2016b) C. Marchant, V. Leiva, F.J.A Cysneiros e J.F. Vivanco. Diagnostics in multivariate birnbaum-saunders regression models. Journal of Applied Statistics, 43:2829-2849. Citado na pág. 2, 10, 14

Ng et al.(2003) H.K.T. Ng, D. Kundu e N. Balakrishnan. Modified moment estimation for the two-parameter birnbaum-saunder distribution. Computational Statistics and Data Analysis, 43: 283-298. Citado na pág. 21

Oh et al.(2008) S. Oh, K.C. Carriere e T. Park. Model diagnostic plots for repeated measures data using the generalized estimating equations approach. Computational Statistics \& Data Analysis, 53(1):222-232. Citado na pág. 24

Park e Lee(2004) T. Park e S.Y. Lee. Model diagnostic plots for repeated measures data. Biom. J., 46:441-452. Citado na pág. 24

Paula et al.(2012) G. A. Paula, V. Leiva, M. Barros e S. Liu. obust statistical modeling using birnbaum-saunders-t distribution applied to insurance. R Applied Stochastic Models in Business and Industry, 28:16-34. Citado na pág. 2, 41

Poon e Poon(1999) W. Poon e Y.S. Poon. Conformal normalcurvature and assessment of local influence. Journal of the Royal Statistical Society, 61:51-61. Citado na pág. 29 
Pregibon(1981) D. Pregibon. Logistic regression diagnostics. Ann Stat, 9:705-724. Citado na pág. 28

Prentice(1988) R. L. Prentice. Correlated binary regression with covariates specific to each binary observation. Biometrics, 44:1033-1048. Citado na pág. 22

Prentice e Zhao(1991) R. L. Prentice e L.P. Zhao. Estimating equations for parameters in means and covariances of multivariate discrete and continuous responses. Biometrics, 47:825-839. Citado na pág. 2

Qu e Xie(2011) Hao Qu e Feng-Chang Xie. Diagnostics analysis for log-birnbaum-saunders regression models with censored data. Statistica Neerlandica, 65(1):1-21. Citado na pág. 53

Rieck(1989) J. R. Rieck. Statistical Analysis for the Birnbaum-Saunders Fatigue Life Distribution. Tese de Doutorado, Department of Mathematical Sciences, Clemson University. Citado na pág. 6

Rieck e Nedelman(1991) J. R. Rieck e J.R. Nedelman. A log-linear model for the birnbaumsaunders distribution. Technometrics, 33:51-60. Citado na pág. 1, 3, 5, 8, 9, 17, 53

Sanhueza et al.(2008) A. Sanhueza, V. Leiva e N. Balakrishnan. The generalized birnbaumsaunders distribution and its theory, methodology, and application. Communications in Statistics - Theory and Methods, 37(5):645-670. Citado na pág. 1

Santos-Neto et al.(2012) M. Santos-Neto, F.J.A. Cysneiros, V. Leiva e S.E. Ahmed. On new parameterizations of the birnbaum-saunders distribution. Pak. J. Statist., 28:1-26. Citado na pág. 1

Santos-Neto et al.(2014) M. Santos-Neto, F. J. A. Cysneiros, V. Leiva e M. Barros. A reparameterized birnbaum-saunders distribution and its moments, estimation and applications. REVSTAT-Statistical Journal, 12(3):247-272. Citado na pág. 1

Song et al.(2004) P. X-K. Song, Z. Qiu e M. Tan. Modelling heteregoneous dispersion in marginal models for longitudinal proportional data. Biometrical Journal, 46:540-553. Citado na pág. 2

Tsionas(2001) E.G. Tsionas. Bayesian inference in birnbaum-saunders regression. Comm. Statist. Theory Methods, 14:179-1932. Citado na pág. 1

Venezuela(2008) M.K. Venezuela. Equação de Estimação Generalizada e Influência Local para Modelos de Regressão Beta com Medidas Repetidas. Tese de Doutorado, Instituto de Matemática e Estatística, Universidade de São Paulo, Brasil. Citado na pág. 2, 13, 17, 24, 29

Venezuela et al.(2007) M.K. Venezuela, D.A. Botter e M.C. Sandoval. Diagnostic techniques in generalized estimating equations. J Stat Comput Simul, 77:879-888. Citado na pág. 24, 28

Venezuela et al.(2011) M.K. Venezuela, M.C. Sandoval e D.A. Botter. Local influence in estimating equations. Comput Stat Data Anal, 55:1867-1883. Citado na pág. 24, 29

Vens e Ziegler(2012) M. Vens e A. Ziegler. Generalized estimating equations and regression diagnostics for longitudinal controlled clinical trials: A case study. Computational Statistics \& Data Analysis, 56(5):1232-1242. Citado na pág. 24

Verbeke(2000) G. Verbeke, G. e Molenberghs. Linear Mixed Models for Longitudinal Data. Springer Series in Statistics. New-York: Springer-Verlag. Citado na pág. 14

Villegas(2010) C. Villegas. Modelos log-Birnbaum-Saunders Mistos. Tese de Doutorado, Instituto de Matemática e Estatística, Universidade de São Paulo, Brasil. Citado na pág. 33, 36 
Villegas et al.(2011) C. Villegas, G.A. Paula e V. Leiva. Birnbaum-saunders mixed models for censored reliability data analysis. IEEE Trans Reliab, 80:748-758. Citado na pág. 2, 6, 9, 14, 17

Wedderburn(1974) R. W. M. Wedderburn. Quasi-likelihood functions, generalized linear models, and the gauss-newton method. Biometrika, 61(3):439. Citado na pág. 2

Xie e Wei(2007) F.C. Xie e B.C. Wei. Diagnostics analysis for log-birnbaum-saunders regression models. Computational Sattistics and Data Analysis, 51:4692-4706. Citado na pág. 53

Zeger e Liang(1986) S.L. Zeger e K-Y Liang. Longitudinal data analysis for discrete and continous outcomes. Biometrics, 42:121-130. Citado na pág. 2

Ziegler(2008) Kastner C. e Blettner M. Ziegler, A. The generalised estimating equations; an annotated bibliography. Biometrical Journal, 40:115-139. Citado na pág. 3, 11 